\title{
THE ROLE OF MONOCYTES IN GOUTY ARTHRITIS
}

By

\author{
(Tommy) Xiao Liu
}

The Malaghan Institute of Medical Research

Wellington, New Zealand

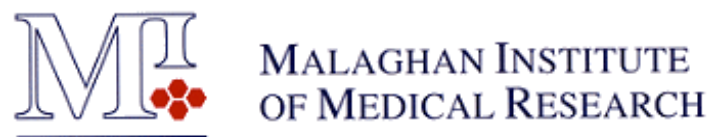

\begin{abstract}
A thesis
submitted to the Victoria University of Wellington in fulfilment of the requirements for the degree of Master of Science in Biomedical Science
\end{abstract}

Victoria University of Wellington June 2009 


\section{Abstract}

Gout is one of the most painful forms of arthritis characterised by the deposition of monosodium urate crystals (MSU) in the joint synovium and the subsequent acute influx of circulating leukocytes. This study investigated the contribution of the inflammatory microenvironment in driving recruited monocyte differentiation and function in acute gouty inflammation.

Using the murine peritoneal model of MSU-induced inflammation, the differentiation and functional phenotypes of MSU-recruited monocytes were compared in normal acute inflammation and in an inflammatory environment depleted of resident macrophages and infiltrating neutrophils. In addition, the role of the local environment in producing monocyte chemoattractant protein-1 (MCP-1) was investigated. Furthermore, the effect of transmigration on the suppressor phenotype of recruited monocytes was also explored.

The pro-inflammatory environment was shown to play a key role in the differentiation and function of recruited monocytes in MSU-induced acute inflammation. In addition, infiltrating neutrophils suppressed the pro-inflammatory abilities of recruited monocytes, which may contribute to the resolution of inflammation. Using both whole peritoneal membrane preparations and in vitro culture techniques, results showed that mesothelial cells lining the peritoneal membrane were a source of $\mathrm{MCP}-1$ production, which contributed to monocyte recruitment. Finally no differences were observed in either the differentiation or functional phenotypes of MSU-recruited monocytes isolated from Glatiramer acetate (GA) treated or non-treated mice.

These findings suggest that the inflammatory microenvironment plays a key role in driving the recruitment, differentiation and function of circulating monocytes in the MSU-induced model of acute inflammation. 


\section{Acknowledgements}

First and foremost, I would like to express my love and gratitude to my parents for their support and patience over this adventure. I don't think I would've been able to do this without them.

Dr Jacquie Harper. Where do I begin? Thanks for opening the doors of research to me; thank you for your support, time, patience and critical feedback; thank you for switching to the blue and black pens on those two rare occasions.

Big thanks to all the past and present minions of the Arthritis group (in no order of favouritism): Willy-John Martin, Rebecca Grainger, Mischa Walton, Stefanie Steiger, Clare Bai/Slaney, and the rest for their invaluable advice and assistance. Thanks for my proofreaders: Evelyn "the-shy-quiet-one-in-the-corner" Spittle and Collin "the monkey" Brooks. Special thanks to Aras Toker and Nina Dickgreber for coming along on coffee breaks, drinking sessions and the overall merriment that occurs whenever they are around.

There have been other people who have been of great help to me; you know who you are but I am mentioning you just in case. Kathryn Farrand, Kate Broadley, and An Tan round off the people in the Oceania office. The BRU staff; without them I wouldn't have any mice to play with. Apii for the "down-the-hall-hi-five" thing that we have going on. Judith Albertson and all the staff at the Newlands Medical Centre. Professor John Miller for his support during my first year of graduate study. Dr Darren Day for letting me do a post-graduate diploma in the first place. The IT gurus Andrew Hamer-Adams and Mike Zablocki for recovering files that continue to become corrupt for no apparent reason. And thanks to everyone else at the Malaghan Institute whom I haven't mentioned for providing such an unbeatable working environment.

Last but not least, special thanks to the otherworldly force that governs the universe. 


\section{Table of contents}

Abstract II

Acknowledgements

Table of contents

List of Figures, Diagrams and Tables $\quad \mathrm{X}$

$\begin{array}{ll}\text { List of abbreviations } & \text { XIII }\end{array}$

CHAPTER 1.0: GENERAL INTRODUCTION 1

$\begin{array}{ll}1.1 \text { GouT } & 2\end{array}$

1.2 ACUTE GOUT 2

1.3 Chronic gout 3

$\begin{array}{lr}\text { 1.4 MONOSODIUM URATE CRYSTALS } & 3\end{array}$

1.5 URIC ACID IS A RISK FACTOR FOR GOUT

1.6 GOUT RISK FACTORS 5

1.6.1 HYPERURICEMIA 5

1.6.2 GENDER 5

1.6.3 DIET 5

1.6.4 CO-MORBID DISEASES

1.6.5 GENETICS 6

1.7 TREATMENT AND MANAGEMENT OF GOUT 6

$\begin{array}{ll}\text { 1.7.1 DIET } & 6\end{array}$

$\begin{array}{lr}1.8 \text { INITIATION OF GOUTY INFLAMMATION } & 6\end{array}$

1.8.1 RECOGNITION OF MSU CRYSTALS AS “DANGER SIGNALS” BY IMMUNE CELLS 7

1.8.2 INNATE CELL RECOGNITION OF MSU CRYSTALS

1.8.3 MACROPHAGES AND THE NALP3 INFLAMMASOME 9

1.8.4 THE PROFILE OF CYTOKINES/CHEMOKINES IN MSU-INDUCED INFLAMMATION 9

1.9 LEUKOCYTE MIGRATION

1.10 NEUTROPHILS IN GOUT

$\begin{array}{ll}1.11 \text { MoNOCYTES IN GOUT } & 13\end{array}$

$\begin{array}{ll}\text { 1.11.1 MONOCYTE SUBPOPULATIONS } & 13\end{array}$

$\begin{array}{ll}\text { 1.11.2 MONOCYTE DIFFERENTIATION } & 14\end{array}$

$\begin{array}{ll}\text { 1.11.3 MONOCYTE ACTIVATION } & 16\end{array}$

$\begin{array}{ll}\text { 1.11.4 RESOLUTION OF GOUT } & 16\end{array}$

$\begin{array}{lc}\text { 1.11.5 MONOCYTE INVESTIGATION } & 18\end{array}$ 
1.12 INTERACTIONS BETWEEN THE INNATE AND ADAPTIVE IMMUNE SYSTEMS 18

$\begin{array}{ll}\text { 1.12.1 SPONTANEOUS ACTIVATION OF T LYMPHOCYTES } & 19\end{array}$

$\begin{array}{lc}1.13 \text { RESEARCH MODELS OF GOUT } & 20\end{array}$

1.13.1 THE MURINE PERITONEAL MODEL OF GOUT 20

1.13.2 MURINE AIR POUCH MODEL 21

1.14 OBJECTIVES OF THIS STUDY 21

CHAPTER 2.0: MATERIALS AND METHODS $\quad 23$

2.1 CHEMiCALS AND REAGENTS

2.2 BUFFERS AND SOLUTIONS

2.2.1 BUFFERS AND MEDIA FOR TISSUE PREPARATION AND CELL CULTURE 24

2.2.2 BUFFERS FOR CELL PURIFICATION 25

2.2.3 BUFFERS FOR FLOW CYTOMETRY 26

2.2.4 SOLUTIONS FOR PREPARATION OF CLODRONATE LIPOSOMES 26

$\begin{array}{ll}\text { 2.2.5 SOLUTIONS FOR IMMUNOHISTOCHEMISTRY } & 27\end{array}$

2.2.6 BUFFERS FOR PROTEIN OR ANTIBODY LABELLING 27

$\begin{array}{lr}2.3 \text { PROTEINS AND COPOLYMERS } & 27\end{array}$

$\begin{array}{ll}2.4 \text { KITS } & 28\end{array}$

2.5 PREPARATION OF MONOSODIUM URATE CRYSTALS

$\begin{array}{ll}2.6 \text { ENDOTOXIN LEVELS } & 30\end{array}$

2.6.1 LIMULUS AMEBOCYTE LYSATE (LAL) ASSAY TEST 30

2.6.2 PURCHASED REAGENTS 31

$\begin{array}{ll}2.7 & \text { ANIMAL STUDIES } \\ & 31\end{array}$

2.7.1 MAINTENANCE AND ETHIC APPROVALS 31

2.7.2 MICE 31

2.7.3 MURINE PERITONEAL MODEL OF MSU-INDUCED INFLAMMATION 31

2.7.4 FLUORESCENT STAINING OF MURINE CD11B ${ }^{+}$BLOOD CELLS IN VIVO 32

2.8 CELL hARVEST AND PROCESSING

2.8.1 PREPARATION OF CELLS FROM PERITONEAL LAVAGE 32

2.8.2 PREPARATION OF CELLS FROM MOUSE BLOOD 33

2.8.3 PREPARATION OF CELLS FROM SPLEENS AND LYMPH NODES 33

2.8.4 PREPARATION OF PRIMARY MOUSE PERITONEAL MESOTHELIAL CELLS 34

2.8.5 CULTURING PERITONEAL MESOTHELIAL CELLS 35

2.9 CELL PURIFICATION

2.9.1 ENRICHMENT OF MOUSE MONOCYTES AND NEUTROPHILS 35 
2.9.2 MAGNETIC SORTING OF CD11 $\mathrm{B}^{+}$CELLS

$\begin{array}{ll}2.10 \text { IN VITRO ASSAYS } & 37\end{array}$

$\begin{array}{ll}2.10 .1 \text { RESTIMULATION ASSAY } & 37\end{array}$

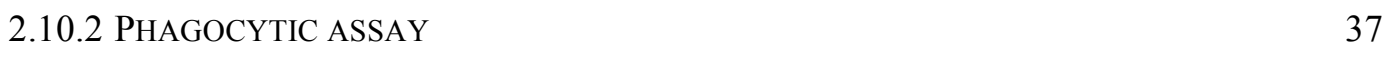

$\begin{array}{ll}2.10 .3 \text { PROLIFERATION ASSAY } & 38\end{array}$

2.10.4 SUPPRESSION ASSAY $\quad 39$

2.10.5 PERITONEAL MESOTHELIAL CELL STIMULATION ASSAY 39

2.10.6 PERITONEAL MEMBRANE STIMULATION ASSAY 39

2.10.7 PREPARATION OF NEUTROPHIL SECRETION $\quad 40$

2.10.8 CULTURING OF MONOCYTES WITH NEUTROPHIL SECRETIONS 40

2.11 FLOW CYTOMETRY 41

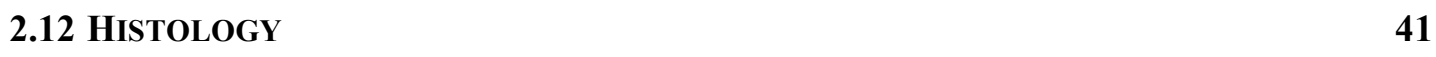

2.12.1 MORPHOLOGICAL HISTOLOGY FOR DIFFERENTIAL CELL COUNTS 41

2.12.2 IMMUNOFLUORESCENT HISTOLOGY 42

2.13 PREPARATION OF CLODRONATE LIPOSOMES

2.13.1 IN VIVO DEPLETION OF RESIDENT MACROPHAGES 43

2.14 FLUOROPHORE LABELLING OF ANTIBODIES AND PROTEINS

2.14.1 ANNEXIN-V AND PROPIDIUM IODIDE (PI) DOUBLE STAINING 44

2.15 IMAGE ANALYSIS AND EDITING 44

$\begin{array}{ll}2.16 \text { STATISTICAL ANALYSIS } & 45\end{array}$

CHAPTER 3.0: THE IMPACT OF THE INFLAMMATORY ENVIRONMENT ON THE DIFFERENTIATION AND FUNCTION OF RECRUITED MONOCYTES IN MSU-INDUCED INFLAMMATION $\quad 46$

$\begin{array}{lr}3.1 \text { INTRODUCTION } & 47\end{array}$

$\begin{array}{ll}\text { 3.1.1 THE INFLAMMATORY ENVIRONMENT IN TUMOURS } & 47\end{array}$

3.1.2 THE INFLAMMATORY ENVIRONMENT IN MULTIPLE SCLEROSIS 48

3.1.3 MONOCYTE RECRUITMENT IN AN MSU-INDUCED INFLAMMATORY ENVIRONMENT48

3.1.4 THE INFLAMMATORY MONOCYTE SUBPOPULATION 49

3.1.5 EFFECTS OF NEUTROPHILS ON THE INFLAMMATORY ENVIRONMENT 49

3.1.6 EFFECTS OF RESIDENT MACROPHAGE DEPLETION ON CELLULAR RECRUITMENT 49

3.1.7 OBJECTIVES $\quad 50$

3.2 RESULTS

3.2.1 PROFILING THE CELL SUBSETS OBSERVED IN MSU-INDUCED INFLAMMATION $\quad 50$ 
3.2.2 DEPLETION OF PERITONEAL RESIDENT MACROPHAGES WITH CLODRONATE LIPOSOMES

3.2.3 EFFECT OF MACROPHAGE DEPLETION ON MSU-INDUCED LEUKOCYTE RECRUITMENT

3.2.4 EFFECT OF MACROPHAGE DEPLETION ON THE PRODUCTION OF MSU-INDUCED CYTOKINES AND CHEMOKINES

3.2.5 CELL MORPHOLOGY OF MSU-RECRUITED MONOCYTES IN NORMAL VERSUS MACROPHAGE-DEPLETED MICE 60

3.2.6 EFFECTS OF MONOCYTE TRANSMIGRATION ON SURFACE MARKER EXPRESSION

3.2.7 CHANGES IN SURFACE MARKER EXPRESSION OF MONOCYTES RECRUITED INTO A MACROPHAGE-DEPLETED ENVIRONMENT

3.2.8 THE EFFECT OF MACROPHAGE DEPLETION ON MSU-RECRUITED MONOCYTE FUNCTION

3.2.9 CHANGES IN PHAGOCYTIC FUNCTION OF MONOCYTES RECRUITED INTO AN ABROGATED INFLAMMATORY ENVIRONMENT

3.2.10 CHANGES IN GROWTH FACTORS DETECTED IN THE LOCAL ENVIRONMENT AFTER DEPLETION OF RESIDENT MACROPHAGES

3.2.11 INFILTRATING NEUTROPHILS CONTRIBUTE TO INFLAMMATION IN MSU-TREATED MICE

3.2.12 SOLUBLE FACTORS PRODUCED BY INFILTRATING NEUTROPHILS SUPPRESS RECRUITED-MONOCYTE ACTIVITY

3.2.13 TGF- $\beta$ SECRETED BY INFILTRATING NEUTROPHILS MAY PLAY A ROLE IN SUPPRESSING RECRUITED MONOCYTE ACTIVITY

\subsection{DISCUSSION}

3.3.1 MSU-INDUCED MONOCYTE RECRUITMENT IN NORMAL AND MACROPHAGEDEPLETED MICE

3.3.2 EFFECT OF THE INFLAMMATORY ENVIRONMENT ON MONOCYTE DIFFERENTIATION AND FUNCTION

3.3.3 THE IMPACT OF COLONY-STIMULATING FACTORS ON RECRUITED MONOCYTE DIFFERENTIATION

3.3.4 NEUTROPHIL SUPPRESSION OF RECRUITED MONOCYTE PR-INFLAMMATORY CAPACITY DURING MSU-INDUCED INFLAMMATION 
CHAPTER 4.0: RECRUITMENT AND GLATIRAMER ACETATE BINDING OF MONOCYTES IN MSU-INDUCED INFLAMMATION

\subsection{MONOCYTE RECRUITMENT BY PERITONEAL MESOTHELIAL CELLS IN MSU-INDUCED} INFLAMMATION

4.1.1 LEUKOCYTE MIGRATION
95

4.1.1.1 Monocyte recruitment in MSU-induced inflammation 95

4.1.1.2 Activation of MCP-1 production by peritoneal mesothelial cells in MSUinduced inflammation

$\begin{array}{ll}\text { 4.1.1.3 Objectives } & 97\end{array}$

$\begin{array}{ll}\text { 4.1.2 RESUlts } & 97\end{array}$

4.1.2.1 MCP-1 production by the peritoneal membrane after MSU or LPS stimulation 97

4.1.2.2 Isolation, identification and culture of peritoneal mesothelial cells 98

4.1.2.3 Peritoneal mesothelial cells produce MCP-1 after MSU or LPS

$\begin{array}{ll}\text { stimulation } & 100\end{array}$

$\begin{array}{ll}\text { 4.1.3 DISCUSSION } & 101\end{array}$

4.2 THE EFFECTS OF GLATIRAMER ACETATE ON RECRUITED MONOCYTES IN MSU$\begin{array}{ll}\text { INDUCED INFLAMMATION } & 104\end{array}$

$\begin{array}{ll}\text { 4.2.1 MONOCYTES IN IMMUNITY } & 104\end{array}$

4.2.1.1 Myeloid-Derived Suppressor Cells 104

4.2.1.2 Glatiramer acetate associates with blood monocytes 104

$\begin{array}{ll}4.2 .1 .3 \text { Objectives } & 105\end{array}$

$\begin{array}{ll}\text { 4.2.2 RESUlts } & 105\end{array}$

4.2.2.1 T cell suppressive ability of MSU recruited monocytes 105

4.2.2.2 GA labelling of circulating monocytes 110

4.2.2.3 $\mathrm{GA}^{+}$monocytes can be recruited into the peritoneum in MSU-induced inflammation 112

4.2.2.4 Effects of GA treatment on T cell suppressive ability of MSU recruited monocytes 113

4.2.2.5 Effects of GA treatment on MSU recruited monocyte phenotype 115

4.2.2.6 Effects of GA treatment on MSU recruited monocyte phagocytic function 116

4.2.2.7 Effects of GA treatment on recruited monocyte functionality 117

$\begin{array}{ll}\text { 4.2.3 DISCUSSION } & 119\end{array}$ 
5.2 EARLY RECRUITED MONOCYTES ARE NON-RESPONSIVE TO MSU RESTIMULATION EX VIVO

5.3 RECRUITED MONOCYTE PHAGOCYTOSIS OF APOPTOTIC NEUTROPHILS AND THE RESOLUTION OF MSU-INDUCED INFLAMMATION.

5.4 IMPACT OF THE INFLAMMATORY ENVIRONMENT ON RECRUITED MONOCYTE DIFFERENTIATION AND FUNCTION 


\section{List of Figures, Diagrams and Tables}

Diagram 1.1: Acute and chronic gouty inflammation. 3

Diagram 1.2: Monosodium urate crystals. 4

Diagram 1.3: Schematic representation of the leukocyte adhesion cascade. 12

Table 2.1: Antibodies used for flow cytometry and immunofluorescent histology. 29

Diagram 2.1: Schematic representation of cell purification by Lympholyte ${ }^{\circledR}-M$ density

gradient.

Figure 3.1: Morphology of cells involved in the peritoneal model of MSU-induced inflammation.

Figure 3.2: Identification of cell types involved in MSU-induced inflammation in vivo.

Diagram 3.1: Schematic representation of clodronate liposome uptake by phagocytic cells.

Figure 3.3: Clodronate liposome depletion of peritoneal resident macrophages.

Figure 3.4: Total cell infiltration after clodronate liposome depletion of resident macrophages.

Figure 3.5: Neutrophil recruitment after resident macrophage depletion in MSUinduced inflammation.

Figure 3.6: Monocyte recruitment after resident macrophage depletion in MSUinduced inflammation.

Figure 3.7: Pro-inflammatory cytokines in the peritoneum after

MSU administration.

Figure 3.8: Pro-inflammatory chemokines in the peritoneum after MSU administration.

Figure 3.9: Morphology of recruited monocytes during the course of an MSU-induced inflammatory response.

Figure 3.10: The process of migrating from the bloodstream through the endothelium alters inflammatory monocyte surface marker expression.

Figure 3.11: Hemopoietic cell surface markers of monocytes recruited into a macrophage-depleted environment.

Figure 3.12: Differentiation cell surface markers of monocytes recruited into a macrophage-depleted environment.

Figure 3.13: Production of pro-inflammatory cytokines by recruited monocytes after restimulation. 
Figure 3.14: Production of pro-inflammatory chemokines by recruited monocytes after restimulation.

Figure 3.15: Phagocytosis of fluorescent beads by monocytes/macrophages.

Figure 3.16: MSU-recruited monocytes from macrophage-depleted mice display reduced phagocytic ability.

Figure 3.17: Phagocytic capacity of monocytes/macrophages.

Figure 3.18: Development of the capacity to phagocytose multiple fluorescent beads by monocytes recruited into a macrophage-depleted environment.

Figure 3.19: Macrophage-Colony Stimulating Factor levels in macrophagedepleted/non-depleted mice during MSU-induced inflammation.

Figure 3.20: Granulocyte-Macrophage-Colony Stimulating Factor levels in macrophage-depleted/non-depleted mice during MSU-inducedinflammation.

Figure 3.21: Co-incubation of neutrophils abrogates monocyte production of proinflammatory cytokines.

Figure 3.22: Co-incubation of neutrophils abrogates monocyte production of proinflammatory chemokines.

Figure 3.23: Pro-inflammatory cytokine production by restimulated monocytes is abrogated by co-incubation with neutrophil-conditioned medium.

Figure 3.24: Pro-inflammatory chemokine production by restimulated monocytes is abrogated by co-incubation with neutrophil-conditioned medium.

Figure 3.25: TGF- $\beta$ suppresses pro-inflammatory cytokine production by recruited monocytes during later stages of MSU-induced inflammation only.

Figure 3.26: TGF- $\beta$ suppresses pro-inflammatory chemokine production by recruited monocytes during later stages of MSU-induced inflammation only.

Figure 4.1: MSU-induced MCP-1 production by the peritoneal membrane is mediated by CD14.

Figure 4.2: Identification of peritoneal mesothelial cells.

Figure 4.3: MCP-1 production by cultured peritoneal mesothelial cells.

Figure 4.4: MSU-induced MCP-1 production by cultured peritoneal mesothelial cells is not mediated by CD14.

Figure 4.5: Monocyte suppression of splenocyte proliferation induced by DynaBead ${ }^{\circledR}$ expander beads.

Figure 4.6: Monocyte suppression of polyclonal $T$ cell proliferation induced by antimouse CD3/CD28 coated plates.

Figure 4.7: Resident macrophage suppression of polyclonal $T$ cell proliferation induced by anti-mouse CD3/CD28 coated plates. 
Figure 4.8: GA binds $C D 11 b^{+}$cell population after i.v. injection.

Figure 4.9: GA binding of blood monocytes is stable over 18 hours.

Figure 4.10: GA bound cells are recruited into the peritoneum after MSU induced inflammation.

Figure 4.11: GA bound monocytes does not alter their suppressive abilities on polyclonal $T$ cell proliferation.

Figure 4.12: GA bound monocytes do not alter their surface marker differentiation profile.

Figure 4.13: GA treatment does not alter phagocytic ability of MSU-recruited monocytes.

Figure 4.14: GA treatment does not alter phagocytic capacity of MSU-recruited monocytes.

Figure 4.15: GA bound monocytes do not alter their capacity to produce cytokines/chemokines after restimulation.

Diagram 5.1: Proposed updates on the MSU-induced inflammation model. 


\section{List of abbreviations}

\begin{tabular}{|c|c|}
\hline ADP & Adenosine diphosphate \\
\hline APC & Antigen presenting cell \\
\hline ATP & Adenosine triphosphate \\
\hline BSA & Bovine serum albumin \\
\hline BMDM & Bone marrow derived macrophage \\
\hline Clodronate & Dichloromethlene diphosphonic acid disodium salt $\left(\mathrm{Cl}_{2} \mathrm{MDP}\right)$ \\
\hline CNS & Central nervous system \\
\hline CSF & Colony stimulating factor \\
\hline DAG & Diacylglycerol \\
\hline $\mathrm{DC}$ & Dendritic cell \\
\hline $\mathrm{ddH}_{2} \mathrm{O}$ & Double-distilled (MilliQ) water \\
\hline DMSO & Dimethlsulfoxide \\
\hline D-PBS & Dulbecco's Phosphate-Buffered Saline \\
\hline EDTA & Ethylene diamine tetra acetate \\
\hline EGF & Epidermal growth factor \\
\hline FACS & Fluorescence activated cell sorter \\
\hline FBS & Foetal bovine serum \\
\hline FITC & Fluorescein isothiocyantae \\
\hline GA & Glatiramer acetate \\
\hline G-CSF & Granulocyte colony stimulating factor \\
\hline GLUT9 & Glucose transporter-9 \\
\hline GM-CSF & Granulocyte macrophage colony stimulating factor \\
\hline Gr-1 & Granulocyte antigen-1 \\
\hline ICAM1 & Intercellular adhesion molecule 1 \\
\hline IFN $\gamma$ & Interferon gamma \\
\hline Ig & Immunoglobulin \\
\hline IL & Interleukin \\
\hline IL-1 & Interleukin-1 \\
\hline IL-1 $\beta$ & Interleukin-1 beta \\
\hline IL-1R & Interluekin-1 receptor \\
\hline IL-6 & Interleukin-6 \\
\hline
\end{tabular}




\begin{tabular}{|c|c|}
\hline $\mathrm{InsP}_{3}$ & Inositol-1,4,5-trisphosphate \\
\hline LPS & Lippolysaccharide \\
\hline $\mathrm{KC}$ & Kertinocyte-derived chemokine \\
\hline MCP-1 & Monocyte chemoattractant protein-1 \\
\hline M-CSF & Macrophage colony stimulating factor \\
\hline MDSC & Myeloid derived suppressor cell \\
\hline MFI & Mean Fluorescence Index \\
\hline MIP & Macrophage inflammatory protein \\
\hline MMP-1 & Matrix metalloproteinase- 1 \\
\hline MSU & Monosodium urate \\
\hline NACHT & $\begin{array}{l}\text { NAIP (neuronal apoptosis inhibitory protein), CIITA (MHC } \\
\text { class II transcription activator), HET-E (incompatibility locus } \\
\text { protein from Podospora anserina) and TP1 (telomerase- } \\
\text { associated protein) }\end{array}$ \\
\hline NADPH & Nicotinamide adenine dinucleotide phosphate (reduced) \\
\hline & NACHT-leucine rich repeat-pyrin domain-containing protein- \\
\hline & 3 (NAPLP)-3 (cryopryin) \\
\hline NO & Nitric oxide \\
\hline NSAIDs & Non-steroidal anti-inflammatory drugs \\
\hline PAMP & Pathogen-associated molecular pattern \\
\hline $\mathrm{PE}$ & Phycoerythrin \\
\hline PECs & Peritoneal exudates cells \\
\hline PerCP & Peridinin chlorophyll protein \\
\hline PFA & Paraformaldehyde \\
\hline PI & Propidium Iodide \\
\hline PLC & Phospholipase C \\
\hline PMCs & Peritoneal mesothelial cells \\
\hline PSGL1 & P-selectin glycoprotein ligand 1 \\
\hline RANKL & Receptor activator of nuclear factor kappa B \\
\hline $\mathrm{RBC}$ & Red blood cells \\
\hline rpm & Revolutions per minute \\
\hline RT & Room temperature \\
\hline SA & Streptavidin \\
\hline
\end{tabular}


TADC

TAM

TG2

TGF- $\beta$

TNF $\alpha$

TLR

URAT1

VCAM1

WBC
Tumour associated dendritic cell

Tumour associated macrophage

Transglutaminase-2

Transforming growth factor- $\beta$

Tumour necrosis factor alpha

Toll-like receptor

Urate transporter-1

Vascular cell-adhesion molecule 1

White blood cell 
Chapter 1.0:

General Introduction 


\section{$1.1 \quad$ Gout}

Gout is one of the most painful forms of arthritis and has a recorded history stretching back several thousand years. It has captured the attention of numerous brilliant physicians over the ages, including the Egyptian Imhotep around 2640BC, and Hippocrates (400BC) whom referred to it as podagara, or "the unwalkable disease" (1).

Originally a disease associated with the wealthy and those of high society (1), the prevalence of gout now strikes across the full range of socio-economic groups, highlighting its emergence as a common disease in western society. The prevalence of gout in New Zealanders of European decent has increased dramatically from 3.1/1000 in 1958 to 29/1000 in $1992(2,3)$. Although not as dramatic, similar increases have been documented in the US where disease prevalence has risen from 2.9/1000 in 1990 to 5.2/1000 in 1999 (4). These statistics emphasize that the incidence of gout is on the rise worldwide.

\subsection{Acute gout}

The deposition of monosodium urate (MSU) crystals in one or more joints causes an acute inflammatory response within the joint synovium resulting in swelling, redness and pain (Diagram 1.1A). These symptoms result from increases in vascular permeability and an intense influx of leukocytes into the local area. Abrupt onset and spontaneous resolution are key characteristics of acute gouty attacks (5) and $60 \%$ of individuals who experience their first gout attack will also experience a second within one year (6). 

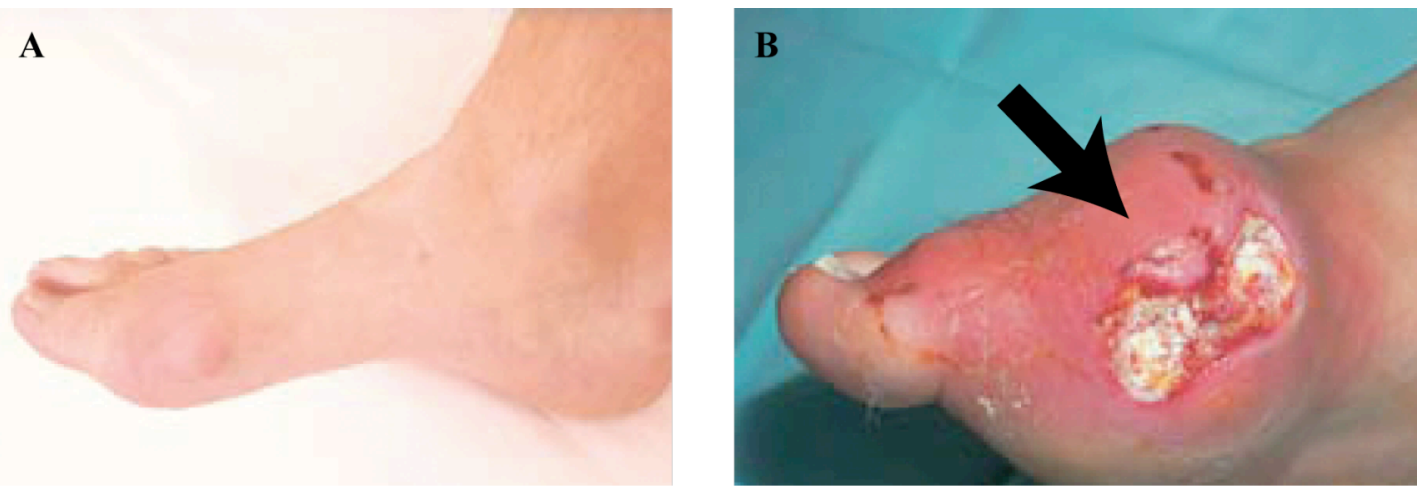

Diagram 1.1: Acute and chronic gouty inflammation. Joints from patients with A. acute gout and B. chronic gout. The arrow indicates the formation of tophi around the joint that have ruptured and caused ulceration through the skin. Photos used with permission from PHARMAC, NZ.

\subsection{Chronic gout}

Poor management of gout may push the disease into a chronic stage. This is characterised by the formation of tophi, masses of MSU crystals and immune cells, which have accumulated in the joints or soft tissue (Diagram 1.1B). These formations around the joints leads to polyarticular bone erosions and deformations (7). Chronic gout is often debilitating with some individuals experiencing periodic attacks of inflammation, known as "gout flares" for the rest of their lives (8).

\subsection{Monosodium urate crystals}

MSU crystals were confirmed as the inducing agent in a gout attack in the 1960s when Faires and McCarty injected synthetically prepared crystals into the synovial cavities of their own knees. The result was a full-fledged gout attack (9).

MSU crystals range in size from 10 to $25 \mu \mathrm{m}$ in length, are needle-like in shape and have the ability to polarize light, a quality known as birefringence (Diagram 1.2). Once precipitated, MSU crystals take a long time to be redissolved by the body and have been identified in the extracted synovial fluid of gout patients even in the absence of clinical inflammation $(10,11)$. 

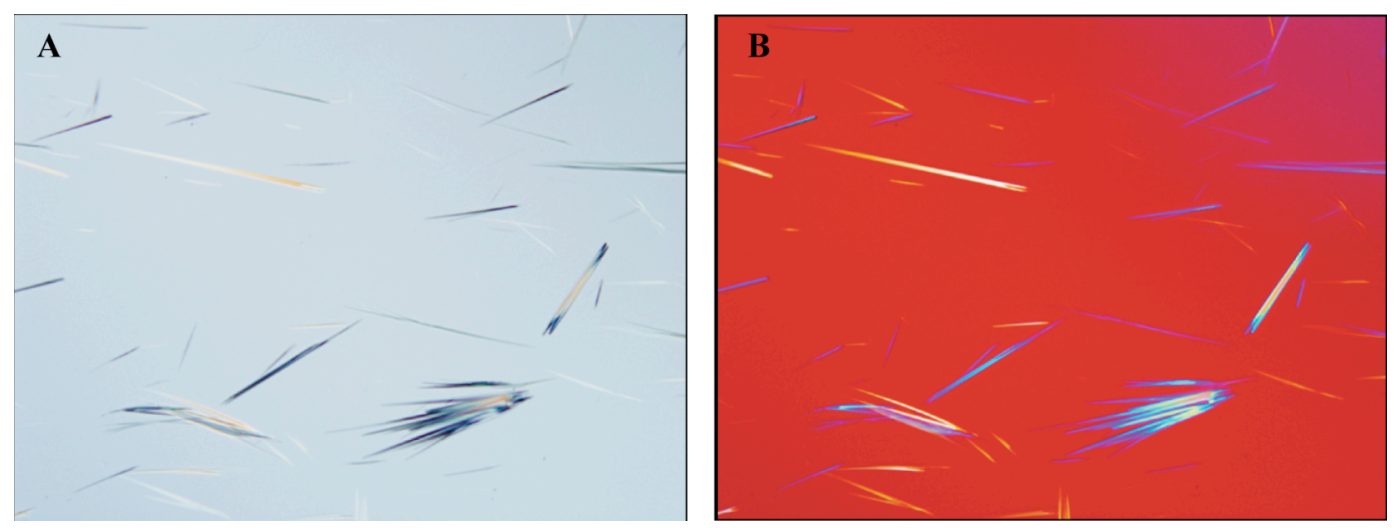

Diagram 1.2: Monosodium urate crystals. Monosodium urate crystals as visualised under a microscope in A. normal and B. polarized light fields. Diagram 1.1 was provided by Dr Willy-John Martin.

\subsection{Uric acid is a risk factor for gout}

Uric acid is a normal by-product of purine breakdown. Proteins, enzymes, and energy carrying molecules (such as NADP and ATP) all require purines as essential building blocks. In humans, renal excretion accounts for approximately $70 \%$ of uric acid excretion and $30 \%$ is removed through the intestine into the faeces (12).

Uricase is an enzyme responsible for the breakdown of uric acid to the more soluble molecule allantoin. The human gene encoding the enzyme was thought to be inactivated due to a mutation sometime during evolution $(13,14)$. Other mammals with functional uricase have significantly lower levels of serum uric acid compared with humans (14), and explains why gout is mainly a human disease.

A range of environmental, genetic and gender factors affect serum uric acid levels, the main predictor of gout risk and development. Normal uric acid levels fall into the range of $2.4-6.0 \mathrm{mg} / \mathrm{dL}$ in women and $3.4-7.0 \mathrm{mg} / \mathrm{dL}$ in men (15). Hyperuricemia is clinically diagnosed when concentrations exceed $7 \mathrm{mg} / \mathrm{dL}$. Previous studies have shown that when uric acid levels approach this concentration, individuals are at risk of MSU crystallization and the onset of gout (16). 


\subsection{Gout risk factors}

\subsubsection{Hyperuricemia}

Even though serum uric acid concentration is thought to be the main predictor of a gout attack, only $25 \%$ of individuals with hyperuricemia develop gout (16). Some individuals can be in a state of asymptomatic hyperuricemia for years without suffering a gout attack. MSU crystals can also be precipitated within the joints between attacks without eliciting an inflammatory response (17). The reasons for these phenomena remain unknown.

\subsubsection{Gender}

Gout is a predominantly male disease with adult men (particularly those aged over 40) being four times more likely than women to develop the disease $(18,19)$. It is believed that the uricosuric effects of estrogen protects women from gout, and is consistent with the observation that women rarely suffer gout attacks prior to menopause (20). Testosterone has also been shown to increase the expression of URAT1, a protein key in urate reabsorption (21).

\subsubsection{Diet}

Intake of purine rich foods (such as seafood, red meat and mushrooms) remains a significant contributor to serum uric acid levels and a major risk factor in gout $(22,23)$. Other foods associated with increased risk include high fructose beverages (such as soft drink and fruit juices), which interfere with ATP recycling, degrading it to uric acid rather than to $\operatorname{ADP}$ then $\operatorname{ATP}(24,25)$. Alcoholic beverages also cause an increase in the rate of purine synthesis within the liver during the metabolism of ethanol $(26,27)$.

\subsubsection{Co-morbid diseases}

Previous studies have made associations between hyperuricemia to not only hypertension but a wide variety of other diseases such as type II diabetes, obesity and cardiovascular diseases (28), collectively known as the metabolic syndrome (29). 


\subsubsection{Genetics}

In humans, genetic mutations causing an overproduction of purines are rare (30); therefore increased genetic risks are more likely due to differences in renal excretion. Recent studies have identified two genes linked to urate reabsorption that have a strong influence on the regulation of serum urate: URAT1 and GLUT9. Polymorphisms in the gene encoding URAT1 have been associated with both hyperuricemia and hypouricemia (31-33). Particular alleles of GLUT9 have been associated with reduced serum urate levels in various populations $(34,35)$.

\subsection{Treatment and management of gout}

There are three main approaches for management of gout. Glucocorticoids and nonsteroidal anti-inflammatory drugs (NSAIDs) can be used to alleviate the immediate inflammatory symptoms associated with an acute gouty attack. Uricostatic drugs (such as allopurinol) elicit their effects by lowering the production of uric acid, while uricosuric drugs (such as benzbromarone) increase renal excretion; both can be used as a long-term treatment for hyperuricemia. However, the easiest approach is a change in lifestyle; more specifically, a moderation of purine rich food intake.

\subsubsection{Diet}

Previous studies have recommended limiting the intake of purine-rich foods and uric acidpromoting beverages (such as alcohol) to prevent increasing serum uric acid levels and the risk of precipitation of MSU crystals $(26,36)$. Other studies have shown that a diet including low-fat dairy products (36), cherries (37), vitamin C (38) and even long-term coffee consumption (39) have been associated with small reductions in serum uric acid concentrations.

\subsection{Initiation of gouty inflammation}

When MSU crystals are deposited within the joint synovium, resident cells recognize and are subsequently activated to initiate an acute inflammatory response. Mast cells, macrophages and epithelial cells have been shown to produce pro-inflammatory molecules 
(such as IL-1 $\beta$, TNF $\alpha$, IL-8, IL-6 and S100 proteins) after MSU-activation (40-43). Complement proteins and various other chemoattractants (such as MCP-1) present in the serum are thought to be involved in the activation and recruitment of circulating inflammatory cells (44). These inflammatory mediators upregulate adhesion molecules on both local the epithelial cells and responding leukocytes, resulting in the recruitment of circulating leukocytes into the joint synovial space.

Neutrophils, once recruited into the synovium during the early phases of an inflammatory response, have been shown to phagocytose MSU crystals, resulting in the production of IL8 and S100 proteins, which leads to further recruitment of leukocytes (45). Other studies have also shown that neutrophils are able to produce destructive reactive oxygen species upon contact with MSU crystals as a part of their programmed responsibilities in the innate immune response (46).

\subsubsection{Recognition of MSU crystals as "danger signals" by immune cells}

Most antigens are poorly- or non-immunogenic by themselves and will not elicit a strong immune response. Therefore an adjuvant or "danger signal" must be present with the foreign particle in order to induce the production of cytokines from resident cells and enhance the overall inflammatory response (47). While lipopolysaccharide (LPS) from Gram-negative bacteria is a well-known danger signal mediated through the Toll-like receptor-4 (TLR4) pathway (48), cell death is also known to trigger adjuvant effects (49, 50). Previous studies have shown that uric acid released from dead cells is associated with causing adjuvant effects and that this process was dependent on MSU crystal formation (51). These results point to uric acid and/or MSU crystals as key endogenous adjuvant/danger signals in the initiation of acute inflammation.

\subsubsection{Innate cell recognition of MSU crystals}

In order for inflammation to occur, innate cells must recognize the MSU crystals. Studies have shown previously that in the joints of complement-deficient rabbits, resident macrophages fail to recognize MSU crystals, resulting in decreased production of the 
neutrophil chemokine kertinocyte-derived chemokine (KC), after urate crystal stimulation (52). In addition, precoating MSU crystals with IgG caused an increase in superoxide production in human polymorphonuclear leukocytes in vitro (46). These results indicate that the recognition of complement proteins on the surface of MSU crystals can lead to the activation of both resident macrophages and infiltrating neutrophils. However, this may not be the only method of recognition, as in the absence of serum, MSU crystals are still able to activate immune cells to produce pro-inflammatory molecules, indicating a more direct recognition of MSU crystals by innate cells (44).

Although the receptors for MSU crystals are not definitively known, there is evidence to support a role for CD14 in crystal recognition. Terkeltaub and colleagues have shown that bone marrow-derived macrophages (BMDMs) from TLR2 and TLR4 knockout mice exhibit impaired recognition and uptake of MSU crystals in vitro (53). Recognition of MSU crystals by the TLR2/TLR4 pathways was also indicated with evidence showing that the TLR4 co-receptor CD14 could bind directly to MSU crystals in vitro (54). In the same study, Liu-Bryan and colleagues showed that the absence of CD14 results in a drastic reduction in both neutrophil recruitment and IL-1 $\beta$ production after MSU crystal stimulation (54).

Contrary to the in vitro data above, Rock and colleagues have shown using a panel of TLR-, IL-1R- and MyD88-deficient mice that the MSU-induced inflammatory response is mediated by IL-1R and the MyD88 pathway but not the TLRs (55). This suggests that although CD14 plays a role in crystal recognition by macrophages, CD14-dependent signalling via the MyD88 pathway rather than TLRs is required for eliciting the production of pro-inflammatory cytokines in response to MSU stimulation. More recently, studies have also shown that macrophage phagocytosis of MSU crystals and subsequent rupturing of intracellular phagosomes is responsible for MSU-induced IL-1 $\beta$ production (56). Therefore these findings indicate that MSU-induced inflammation might occur through more than one pathway. Nevertheless, with the identification of the importance of MSU crystals in the initiation of innate inflammation, the recognition processes for crystals remain the focus of extensive research. 


\subsubsection{Macrophages and the NALP3 inflammasome}

Macrophages are the linchpin of innate immunity and are found throughout the tissues of the body, including Kupffer cells of the liver, alveolar macrophages of the lung, serosal macrophages of the peritoneum, type A cells in the joint synovium, and microglia of the central nervous system (CNS) (57). They play an important role in the clearance of pathogens through their phagocytic ability. Upon stimulation with MSU crystals in vitro, resident macrophages produce pro-inflammatory cytokines (including IL-1 $\beta$, TNF $\alpha$, IL18) and iNOS $(55,58-60)$. Previous studies have shown that the blockade of IL-1 $\beta$ abrogates inflammation, highlighting a key role for IL-1 $\beta$ in gouty inflammation (55).

The cleaving of preformed intracellular stores of pro-IL-1 $\beta$ into activated IL- $1 \beta$ requires the assembly of the NACHT-LRR-PYD-containing protein-3 (NALP3) inflammasome (61). This multi-protein complex is comprised of the intracellular pattern recognition receptor NALP3, the accessory protein ASC, and procaspase-1 (60). Following activation, NALP3 oligomerises and recruits ASC, then binds to procaspase-1 resulting in autocatalytic processing and activation (62). Caspase-1 activation leads to pro-IL-1 $\beta$ cleavage and the release tof mature pro-inflammatory IL-1 $\beta$ from the cell (61).

Chen and colleagues have shown previously that IL- $1 \beta$ production and IL- $1 \beta$ receptor (IL1R) activation are essential for neutrophil recruitment and MSU-induced inflammation, using a panel of knockout mice with deficiencies in the inflammasome (such as ASC, caspase-1 and NALP3) and the IL-1R (55). In addition, the blockade of IL-1R resulted in the resolution of gout symptoms in patients treated with anakinra - a recombinant version of IL-1 receptor antagonist. Together, these findings indicate the importance of the inflammasome and of IL- $1 \beta$ in gout.

\subsubsection{The profile of cytokines/chemokines in MSU-induced inflammation}

Previous studies in our group have shown that administration of MSU crystals via intraperitoneal injection induces the production of IL-1 $\beta$, IL-6, TNF $\alpha$ and MCP-1 (also known as CCL2) from activated resident macrophages (58). In addition MSU-elicited 
leukocyte recruitment peaked at 18 hours after MSU administration, with the monocytes recruited at a slower rate and in smaller numbers compared to the infiltrating neutrophils (58).

In humans, neutrophils recruited into inflamed tissue produce large amounts of the chemokine IL-8, resulting in neutrophil priming and further recruitment via the IL-8 receptor (CXCR-2) $(63,64)$. As mice do not have the gene that encodes IL-8 (65), previous studies of animal models of gout have shown a requirement for the neutrophil chemokine $\mathrm{KC}$, which is also known as chemokine ligand 1 (CXCL1), and IL-1 $\beta(55,60)$ for neutrophil recruitment while other molecules such as TNF $\alpha$, GM-CSF (66), S100A9 and S100A9 (67) and complement proteins (44) are believed to supplement immune cell recruitment. MSU-activated monocytes have been shown to produce mediators such as TNF $\alpha$ and IL-1 $\beta$ to upregulate adhesion molecules E-selectin, ICAM-1 and VCAM-1 on human endothelial cells, allowing circulating neutrophils to adhere to the endothelium in vitro (68). These studies indicate that the recruitment of neutrophils is dependent on a number of cell types that collectively contribute to the neutrophil response in MSUinduced acute inflammation.

While the monocyte chemoattractant MCP-1 was thought previously to be produced by macrophages (69), our studies have shown that depletion of macrophages prior to MSU administration significantly abrogated neutrophil but not monocyte recruitment (58). This indicated that while macrophages play a key role in neutrophil recruitment via the production of the chemokine $\mathrm{KC}$, other resident cells regulate the production of MCP-1. It has been shown previously that MCP-1 can be detected within the synovium of rabbit joints after MSU administration (70). Furthermore, in the same study, MCP-1 was detected in synovial lining cells using immunohistochemistry (70). Therefore, further research is required to investigate whether the local membrane cells are responsible for MCP-1 production and monocyte recruitment. 


\subsection{Leukocyte migration}

Monocyte and neutrophil recruitment is a key characteristic of acute inflammation in gout. The movement of leukocytes from the bloodstream to sites of inflammation is a strictly controlled process involving regulated steps collectively known as the adhesion cascade (71) (Diagram 1.3).

Leukocyte rolling is mediated by various selectins (such as L-selectin on leukocytes and Eselectin on inflamed endothelial cells) interacting with P-selectin glycoprotein ligand-1 (PSGL1) and other co-molecules that are expressed on endothelial cells (72). Binding of PSGL1 and selectins causes leukocyte adherence to inflamed endothelium in a pulse like fashion, with exceptionally high on- and off-rates of adherence which determines the speed at which leukocytes are 'rolling' (71).

Chemokines are a group of small molecules expressed by endothelial cells, which are responsible for initiating the arrest of 'rolling' leukocytes within the adhesion cascade. Monocyte chemoattractant protein-1 (MCP-1) is one such molecule and has been associated with the recruitment of monocytes through the endothelium in rheumatoid arthritis (73). MCP-1 binds with high affinity to specific G-protein transmembrane receptors on rolling monocytes, which results in rapid activation of phospholipase $\mathrm{C}$ (PLC), leading to an increase in intracellular $\mathrm{Ca}^{2+}$ and generation of inositol-1,4,5trisphosphate $\left(\mathrm{Ins}_{3}\right)$ and diacylglycerol (DAG) which are all required to initiate cell arrest (74). Previous findings have shown that the binding of leukocyte integrins to transmembrane proteins such as intercellular adhesion molecule-1 (ICAM1) and vascular cell-adhesion molecule-1 (VCAM1) $(75,76)$ are responsible for cell arrest and contribute to the strengthening of adhesion prior to migration from the blood vessels (74).

Three key mechanisms have been identified by which leukocytes may transmigrate through the endothelium; crawling, paracellular and transcellular migration (71). Crawling is performed by leukocytes seeking preferred sites of transmigration. This process involves the extension of leukocyte membrane protrusions into the endothelial cell body and triggering the ligation of ICAM1 by macrophage antigen-1 (MAC1). Eventually this leads to enhanced endothelial cell contraction and opening of inter-endothelial contacts (77). 
Paracellular migration involves the ligation of endothelial-cell adhesion molecules leading to reduced inter-endothelial contacts and therefore allowing the migration of leukocytes through endothelial cell-junctions. This process is facilitated by a gauntlet of interactions between leukocyte membrane compartments containing platelet/endothelial-cell adhesion molecules and cell junctions on the endothelium (77). The transcellular route is believed to occur in thin endothelium such as the central nervous system (CNS) (78) whereby leukocytes transmigrate through an intracellular channel within the endothelial cell mediated by ICAM1 ligation (79).

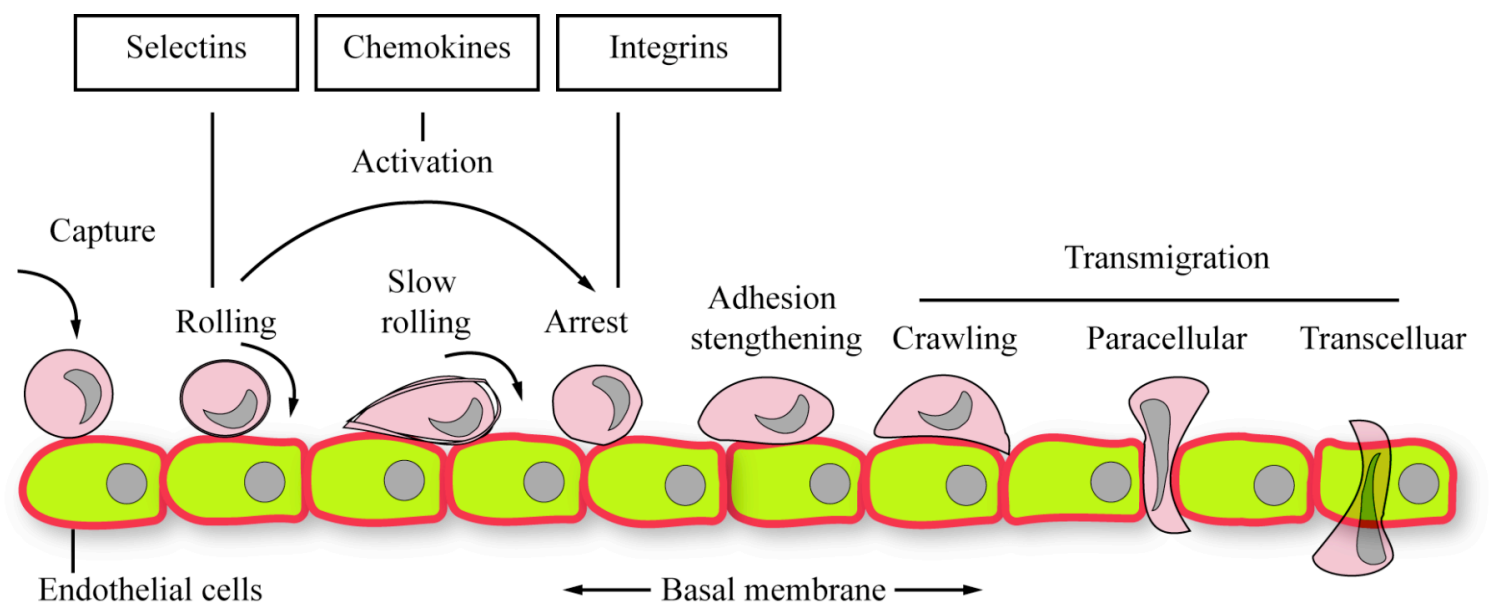

Diagram 1.3: Schematic representation of the leukocyte adhesion cascade. Rolling is mediated by selectins, activation is mediated by chemokines, and arrest, is mediated by integrins. Transmembrane migration can be separated into crawling, paracellular and transcellular migration. Diagram 1.3 was adapted from (71).

\subsection{Neutrophils in gout}

Neutrophils, also known as polymorphonuclear leukocytes (PMNs), comprise $40-70 \%$ of circulating leukocytes in humans. Under homeostatic conditions, following release from the bone marrow, neutrophils undergo apoptosis within $6-10$ hours $(80,81)$ and are cleared from the circulation by the liver and splenic macrophages (82).

Neutrophils are a part of the host's innate immune defence and are among the first leukocytes recruited into inflamed tissues. Once recruited to a site, their role involves the 
uptake of infectious matter, pathogens and debris, and production of hazardous molecules to kill and digest foreign organisms and remove damaged tissue (83).

Studies have shown previously that neutrophil recognition of foreign particles, bacteria or other inflammatory agents is mediated through pattern recognition receptors (such as TLRs) (84). Furthermore, foreign agents may become opsonised with antibodies and complement factors, which may be detected and phagocytosed by neutrophils through interactions with $\mathrm{F}_{\mathrm{c}}$ and complement receptors (85).

Neutrophil recognition and phagocytosis of inflammatory particles activates the respiratory burst enzyme, NADPH oxidase which leads to the production of extracellular superoxide from the cell that can lead to further bactericidal activity and tissue damage (86). In addition, neutrophils are able to produce pro-inflammatory cytokines (such as IL-8 (87) and S110A8/A9 (67)), which induces further neutrophil recruitment and amplifies the inflammatory response.

Within the inflamed tissue, exposure to cytokines (such as IL-1 $\beta$, TNF $\alpha$ and IFN $\gamma$ ), growth factors (such as G-CSF and GM-CSF) and MSU crystals have been shown to activate and subsequently prolong the half-life of recruited neutrophils from hours to days in vitro (88). This may result in an accumulation of normally short-lived neutrophils within the inflamed environment in order to induce a prolonged pro-inflammatory response.

In gout, neutrophils have been shown to be one of the main recruited leukocytes after MSU-crystal deposition within the joint (58) and play a key part in inducing the overall acute inflammatory response.

\subsection{Monocytes in gout}

\subsubsection{Monocyte subpopulations}

In $\mathrm{C} 57 \mathrm{~B} 1 / 6 \mathrm{~J}$ mice, circulating monocytes are primarily identified by their expression of CD11b, low levels of F4/80, and intermediate levels of Gr-1 (Ly6C and $\mathrm{Ly}^{-} \mathrm{G}^{+}$) (89). To 
date, two circulating monocyte subpopulations (expressing either Gr- $1^{\text {low }}$ or $\mathrm{Gr}-1^{\text {hi }}$ ) have been shown to reside in the blood (90). In mice, the former is further characterised as resident $\mathrm{CX}_{3} \mathrm{CR} 1^{\mathrm{hi}} \mathrm{CCR} 2^{-} \mathrm{Gr}^{-}$monocytes due to their ability to remain within the bloodstream during homeostasis, and take-up a "patrolling" role $(90,91)$. Under steadystate conditions, this subpopulation migrates between the bloodstream and the local tissue through the endothelium and "samples" the environment for danger signals (90). Further studies have found this migratory observation to be dependent on Fractalkine ( $\left.\mathrm{CX}_{3} \mathrm{CL} 1\right)$, a transmembrane chemokine expressed on endothelial cells, the ligand for $\mathrm{CX}_{3} \mathrm{CR} 1$ (91). Adoptive transfer studies have shown previously that $\mathrm{Gr}-1^{-}$monocytes migrate into tissues under homeostatic conditions and are believed to replenish resident cell populations under steady state conditions (91).

The Gr- $1^{\text {hi }}$ circulating “inflammatory” monocytes display a $\mathrm{CX}_{3} \mathrm{CR} 1^{\text {low }} \mathrm{CCR} 2^{+}$phenotype and are recruited to inflamed peripheral tissue by MCP-1, the ligand for CCR2 (57, 90, 91). Although inflammatory monocytes can differentiate into both macrophages and dendritic cells (DCs) in vitro, Auffray and colleagues has shown that inflammatory monocytes are predisposed to becoming DCs following LPS insult in vivo $(90,92)$. Alternatively, Chazaud and colleagues have shown that after skeletal muscle injury in mice, recruited monocytes differentiate into $\mathrm{F} 4 / 80^{\mathrm{hi}}$ macrophages to support myogenesis (93). Together, these findings indicate that the fate of differentiation of this particular subset of circulating monocytes is dependent on the type of stimulus and the inflammatory environment into which they are recruited.

\subsubsection{Monocyte differentiation}

Monocytes are phagocytic mononuclear cells that comprise approximately $2-10 \%$ of white blood cells in circulation in humans. Although they have been shown previously to produce pro-inflammatory mediators such as IL-8, TNF $\alpha$ and IL-6 after LPS stimulation in vitro, they are considered to be an undifferentiated myeloid precursor cell (94). Once monocytes are recruited from the bloodstream into peripheral tissues, they have the capacity to differentiate into a number of cell types depending on the soluble mediators present within the local environment. The influence of the inflammatory environment on 
the differentiation of recruited monocytes has been shown in a wide variety of disease models. For example, mononuclear precursors recruited into a tumour environment containing anti-inflammatory molecules (such as glucocorticoid hormones, IL-4, IL-13 and IL-10) are programmed to differentiate into alternatively activated macrophages, also known as tumour associated macrophages (TAMs) $(57,95,96)$. TAMs have been shown to produce anti-inflammatory cytokines (such as IL-10 (97) and TGF- $\beta$ (98)) and contribute to immune-tolerance and tumour angiogenesis (98). Alternatively, in the central nervous system (CNS) of experimental autoimmune encephalomyelitis (EAE) mice rich in IFN- $\gamma$, TNF $\alpha$ (99) and IL-17 (100, 101), recruited myeloid precursors have been shown to develop macrophage-like phenotypes (102) and produce reactive oxygen species (ROS) and other pro-inflammatory cytokines (103), leading to demyelination and axonal damage (104).

Granulocyte/macrophage-stimulating factor (GM-CSF, also known as CSF2) and macrophage-stimulating factor (M-CSF, also known as CSF1) are haematopoietic-cell growth factors defined by their abilities to generate colonies of mature myeloid cells from bone-marrow precursor cells in vitro. Warren and Vogel have shown that while monocytes cultured in M-CSF predominantly differentiate into macrophages (105), monocytes cultured in GM-CSF and IL-4 become dendritic cells (DCs) (106). Furthermore, monocytes cultured with RANKL and M-CSF differentiate into osteoclasts in vitro (107). More recently, M-CSF and IL-10 in the tumour environment have been proposed to programme the differentiation of recruited monocytes into TAMs by blocking their differentiation into DCs $(96,108)$. Higher concentrations of GM-CSF detected within bronchoalveolar lavage fluid have also been proposed to generate an unique population of DC-like macrophages in vivo (109). Together, these findings indicate a role for colony stimulating factors (CSFs) in determining the fate of recruited myeloid precursor cells. 


\subsubsection{Monocyte activation}

The activation of monocytes and macrophages can induce differential responses depending on the stimulus (57). Three common activation responses have been identified, based on in vitro activation of macrophages with different stimuli.

Classical activation occurs by the triggering of inflammatory responses by stimuli such as TLR ligands. Classically activated macrophages activated by pathogen-associated molecular patterns (PAMPs) such as LPS produce large amounts of pro-inflammatory cytokines (such as IFN- $\gamma$, IL-6 and TNF $\alpha$ ) and reactive oxygen species (ROS); have an enhanced capacity for antigen presentation and increased microbicidal activity $(57,89,95)$. Alternative activation by anti-inflammatory cytokines (i.e. in the presence of IL-4 and IL13) results in the promotion of tissue repair, low level production of anti-inflammatory cytokines (such as TGF $\beta$ ) and increased parasite killing $(57,95)$. In addition, type II activation of macrophages by immune complexes (e.g. Ig-opsonised antigens) drives the production of high levels of IL-10, increased nitric oxide (NO) production and enhanced presentation of antigens (95).

Monocyte activation appears to also depend on environmental stimuli; LPS-activated monocytes exhibit a similar pro-inflammatory profile to classically activated macrophages by their production of pro-inflammatory cytokines (such as IFN $\gamma$ and IL-6) via the MyD88 pathway (55). The differentiation of monocytes into macrophages in vivo may be associated with different types of activation.

\subsubsection{Resolution of gout}

Although the mechanism behind the spontaneous resolution of gout is not definitively known, there is evidence to indicate that mononuclear phagocytes mediate resolution of acute inflammation via the production of anti-inflammatory cytokines (such as TGF- $\beta$ ).

Barin and colleagues have shown previously that recombinant human transforming growth factor- $\beta$ (TGF- $\beta$ ) was able to suppress the inflammatory response induced by MSU administration, in a rat subcutaneous air pouch model (110). However, while showing that 
TGF- $\beta$ has an inhibitory effect on MSU-induced inflammation, this study did not identify a potential source of TGF- $\beta$ production.

Experiments by the Haskard group have shown using a panel of murine monocyte/macrophage cell lines that monocyte-like cells produced the pro-inflammatory cytokine TNF $\alpha$ after MSU stimulation in vitro, while mature macrophage-like cells were non-inflammatory when exposed to MSU crystals (111). In a followup experiment by the same group using human peripheral blood monocytes, differentiated by adherence in vitro over $1-7$ days, Haskard and colleagues showed that undifferentiated blood monocytes produced IL-6, IL-1 $\beta$ and TNF- $\alpha$ in response to MSU stimulation in vitro, while mature macrophages showed reduced levels of these pro-inflammatory cytokines and instead produced large amounts of TGF- $\beta(112,113)$. While these studies showed the importance of the stage of monocyte/macrophage differentiate in the production of "pro"- or "anti"inflammatory cytokines after MSU stimulation in vitro, the relevance of these findings relative to the inflammatory environment in vivo is not clear.

Further studies on the phagocytosis of apoptotic neutrophils by macrophages have indicated a role in the production of anti-inflammatory cytokines, contributing to the resolution of inflammation. Terkeltaub and colleagues have shown in $\mathrm{TG} 2^{-/-}$mice that are defective in the ability to phagocytosis apoptotic cells, that MSU administration i.p. exhibited a prolonged and more intense neutrophil infiltration compared with wild-type mice. This indicated that failed uptake of neutrophils by macrophages resulted in unresolved acute inflammation. Finally, in a previous study, human monocyte-derived macrophages that ingested apoptotic neutrophils produced anti-inflammatory mediators (including TGF- $\beta$ ) after LPS stimulation, while downregulating pro-inflammatory cytokines (such as $\mathrm{TNF} \alpha$ ) in vitro.

Although these studies together establish a model of resolution that incorporates macrophages switching to an anti-inflammatory phenotype after phagocytosis of apoptotic neutrophils and the involvement of TGF- $\beta$ as a suppressive cytokine, whether these mechanisms actually occur in gout has yet to be determined in vivo. 
A clear limitation of the in vitro studies is that they do not account for the functional changes that occur during cellular recruitment. As circulating monocytes transmigrate through the endothelium, ligand interactions result in an increase in the expression of several genes (including MMP-1, MCP-1, TG2 caveolin-1 and CD4) (114). Therefore, the in vitro conditions (using only M-CSF, GM-CSF or by adherence to culture plates) used in previous studies are completely different to the inflammatory environment (consisting of a complex mixture of cytokines/chemokines, serum, cells, and tissues) into which monocytes are recruited in vivo (115).

\subsubsection{Monocyte investigation}

Since recruited monocytes play an important role in both the "pro"- and "anti"- phases of inflammation, the mechanism that drives their differentiation and function has been a subject of interest in gout research. Common research approaches include the use of immortalized murine monocyte/macrophage cell lines or the differentiation of human peripheral blood monocytes in vitro, yet monocytes are never isolated during an inflammatory response. Therefore, a major area of investigation is how monocytes fit into the network of responses during an inflammatory response, working in concert and cooperation with other environmental/cellular mediators, such as neutrophils.

\subsection{Interactions between the innate and adaptive immune systems}

The innate and adaptive immune systems have been traditionally considered as separate but complementary mechanisms of defence against pathogenic challenges. However, advances in the understanding of the nature and functions of TLRs have sparked new appreciation of the interdependence and cross-talk that occurs in an immune response and has given rise to renewed interest in the interactions between the innate and adaptive branches of the immune network.

The common explanation for the death of lymphocyte-deficient hosts would be their lack of an adaptive immune response to effectively clear pathogens and therefore avoid acute infection (116). However, $\mathrm{Fu}$ and colleagues have shown that an un-regulated innate 
immune response in the form of a cytokine storm can also be a direct cause of death, after viral infection in T-cell- and lymphocyte- deficient mice (117). In addition, studies have shown recently that both effector and memory CD4+ T cells suppressed macrophage inflammasome-mediated caspase-1 activation and subsequent IL-1 $\beta$ release, using a variety of murine peritonitis models (including MSU crystals, alum and asbestos) (118). Furthermore, myeloid-derived suppressor cells (MDSCs) have been shown to inhibit T cell responses in tumour models (119), which may be an exacerbated phenotype of the baseline suppressive mechanism mediated by MDSCs during homeostasis. Nevertheless these findings may indicate a cross-regulatory role between the innate and adaptive systems, which may be necessary to temper immune responses and potentially control non-specific activation.

\subsubsection{Spontaneous activation of $T$ lymphocytes}

The cellular branch of adaptive immunity is mediated by $\mathrm{T}$ cells, which express antigen specific receptors (TCRs) and combat mostly intracellular pathogens (120). Circulating T cells can be activated by antigen presenting cells (APCs) (such as DCs) in lymphoid organs and differentiate into effector $\mathrm{T}$ cells. Based on $\mathrm{T}$ cell function and surface marker expression, they can be categorized into cytotoxic T lymphocytes (CTL also known as CD8 $\mathrm{T}$ cells) and $\mathrm{T}$ helper cells $\left(\mathrm{T}_{\mathrm{H}}\right.$ cells or CD4 T cells). CD8 $\mathrm{T}$ cells are responsible for inducing the apoptosis of infected host cells through granzymes or Fas-FasL interactions (120). CD4 T cells produce pro-inflammatory cytokines that can potentiate CTL responses or promote humoral immunity (121).

Studies have shown previously that spontaneous $\mathrm{T}$ cell proliferation can occur when naïve CD4 T cells are transferred into lymphopenic mice (122). In the same study, Paul and colleagues were able to show that "homeostatic proliferation" can occur by two distinguishable mechanisms, which they proposed might indicate fundamentally distinct roles in the maintenance of CD4 $\mathrm{T}$ cell homeostasis (122). In addition, spontaneous lymphocyte proliferation was observed in isolated CD4 and CD8 T cells from patients infected with T-cell lymphotropic virus type I $(123,124)$. These findings may indicate a possible non-antigen specific, spontaneous proliferation of resident $\mathrm{T}$ cells, which is only 
highlighted in an immune-compromised or artificial setting. Whether cells of the innate immune system play a role in the regulation of this background spontaneous $\mathrm{T}$ cell proliferation warrants further research.

\subsection{Research models of gout}

Research models of disease are essential in order to study acute gout or any other form of inflammatory disease. Faires and McCarty developed the first gout animal model that resulted in significant observations, whereby they injected synthetic MSU crystals into dog knees (9). The authors were able to replicate the same inflammatory symptoms that they observed when they self-administered MSU crystals into their own knees, such as pain, swelling and redness (9).

Since then, mouse and rat models have been developed in order to study gouty inflammation. As the sizes of murine joints are too small to make intrasynovial injections of MSU crystals, within murine models, alternative tissue sites are used to mimic the joint synovium.

\subsubsection{The murine peritoneal model of gout}

The peritoneal model of gout was first used in research by Getting and colleagues (42). The peritoneal cavity contains cell subsets in vivo similar to those in the joint synovium, including macrophages, epithelial cells, mast cells, fibroblasts and lymphocytes. Similar to the inflammatory response in gout, the injection of MSU crystals into the peritoneum induces a robust leukocyte infiltration (including neutrophils and monocytes), as well as the production of cytokines and chemokines. Limitations of this model include the absence of certain cell types such as osteoclasts and chondrocytes specific to the bone niches, as well as tissue extracellular matrixes such as cartilage and bone, which are more relevant for models of chronic gout. The peritoneal model is also limited by its relatively large size, with the cavity lacking the mechanically induced algesia due to oedema normally accompanied during a gout attack. Despite the disadvantages above, outcomes observed from this model has been used extensively through to clinical studies of the disease, confirming its usefulness and relevance in gouty inflammation. 


\subsubsection{Murine air pouch model}

The murine air pouch model is another research model of acute gout. A subcutaneous injection of sterile air on the back of rats (or mice) is performed in order to produce an air sac. With the air pouch mimicking the joint synovium, an MSU challenge induces the recruitment of leukocytes. This model has been used as an MSU-induced recruitment model of acute gout in previous studies (125).

The limitations of this particular model are similar to the peritoneal model; with an absence of cell subtypes normally found in the bone niche. In addition, the initial inflammation caused by the formation of the air pouch may cause an inflammatory background of altered cell numbers or cell phenotype, which may modify changes observed in subsequent inflammatory changes.

Therefore, the MSU-induced peritoneal inflammatory model was chosen for this research. It induces an un-altered inflammatory background with cellular infiltration, which mimics clinical gout. It is supported by the model's use in literature and relevancy in clinical translational experiments.

\subsection{Objectives of this study}

The research introduced has summarised the roles of infiltrating neutrophils, monocytes and macrophages in acute gout. My thesis aims to assess the effect of the recruiting inflammatory environment as well as the changes in the differentiation and functions of monocytes recruited into the peritoneum by MSU-induced inflammation.

The objectives of this study were:

Aim 1: $\quad$ To profile changes in recruited monocyte differentiation and functionality after the depletion of resident macrophages in MSUinduced inflammation. 
Aim 2: $\quad$ To investigate the impact of leukocyte transmigration on the suppressor phenotype of recruited monocytes.

Aim 3: $\quad$ To identify the cell subset responsible for the production of MCP-1 during MSU-induced inflammation. 
Chapter 2.0:

Materials and Methods 


\subsection{Chemicals and reagents}

Chemicals were purchased from the following companies as listed in section 2.2:

BDH Chemicals, NZ

Invitrogen, $\mathrm{NZ}$

Merck, Australia

PAA, Austria

Roche, NZ

Sigma-Aldrich, NZ

\subsection{Buffers and solutions}

\subsubsection{Buffers and media for tissue preparation and cell culture} Complete RPMI-1640 (cRPMI) media

$\begin{array}{lll}\text { Foetal Bovine Serum (FBS) } & 10 \%(\mathrm{v} / \mathrm{v}) & \text { Sigma-Aldrich, } \\ & & \text { Invitrogen } \\ \text { Glutamax } & 2 \mathrm{mM} & \text { Invitrogen } \\ \text { Penicillin-streptomycin } & 100 \mathrm{U} / \mathrm{mL} & \text { Invitrogen }\end{array}$

In sterile RPMI-1640 (Invitrogen). Stored at $4^{\circ} \mathrm{C}$ and used sterile.

\section{Collagenase II/DNase I solution}

Collagenase Type II

$1 \mathrm{mg} / \mathrm{mL} \quad$ Invitrogen

DNase I

$0.2 \mathrm{mg} / \mathrm{mL}$

Roche

In sterile cRPMI. Filter sterilized using $32 \mathrm{~mm}$ syringe filters with $0.2 \mu \mathrm{m}$ Supor ${ }^{\circledR}$ membrane (Pall Corporation, Newquay, Cornwall, UK), stored at $4^{\circ} \mathrm{C}$ and used sterile.

\section{Peritoneal Mesothelial Cell (PMC) growth media}

$\begin{array}{lll}\text { Epidermal Growth Factor } & 20 \mathrm{ng} / \mathrm{mL} & \text { Roche } \\ \text { FBS } & 10 \%(\mathrm{v} / \mathrm{v}) & \text { Sigma-Aldrich, } \\ & & \text { Invitrogen } \\ \text { Glutamax } & 2 \mathrm{mM} & \text { Invitrogen } \\ \text { Hydrocortisone } & 5 \mu \mathrm{M} & \text { Sigma-Aldrich }\end{array}$


Penicillin-streptomycin

$100 \mathrm{U} / \mathrm{mL}$

Invitrogen

In sterile RPMI-1640 (Invitrogen). Stored at $4^{\circ} \mathrm{C}$ and used sterile.

\section{Dulbecco's Phosphate Buffered Saline (D-PBS) in-house}

D-PBS powder

$95.5 \mathrm{~g} /$ package

Invitrogen

Made up to 10 litres in MilliQ water (Q-Grad® 1 Purification Pack, Millipore Corp., Billerica, MA, USA), pH adjusted to 7.1. Filter sterilized using $0.22 \mu \mathrm{m}$ vacuum driven disposable bottle top filters (Steritop ${ }^{\mathrm{TM}}$ Millipore Corp., Billerica, MA, USA), stored at $4^{\circ} \mathrm{C}$ and used sterile. This D-PBS was used for procedures that did not require D-PBS to be endotoxin free.

\section{Red Blood Cell (RBC) lysis buffer}

Solution 1

$\mathrm{NH}_{4} \mathrm{Cl} \quad 0.16 \mathrm{M} \quad$ Merck

Solution 2

Tris $\quad 0.17 \mathrm{M} \quad$ Invitrogen

In MilliQ water. Autoclave sterilized, stored at room temperature (RT) and used sterile. Working RBC lysis buffer was prepared by mixing Solutions 1 and 2 at a 9:1 ratio immediately before use.

\section{Serum-free RPMI-1640 media}

Glutamax

$2 \mathrm{mM}$

Invitrogen

Penicillin-streptomycin

$100 \mathrm{U} / \mathrm{mL}$

Invitrogen

In sterile RPMI-1640. Stored at $4^{\circ} \mathrm{C}$ and used sterile.

\subsubsection{Buffers for cell purification}

autoMACS ${ }^{\mathrm{TM}}$ rinsing buffer

EDTA

$2 \mathrm{mM}$

Invitrogen

In Dulbecco's Phosphate Buffered Saline (D-PBS, Invitrogen). Autoclave sterilized, stored at $4^{\circ} \mathrm{C}$ and used sterile. 
autoMACS ${ }^{\mathrm{TM}}$ running buffer

FBS

$1 \%(\mathrm{v} / \mathrm{v})$

PAA

EDTA

$2 \mathrm{mM}$

Invitrogen

In D-PBS. Autoclave sterilized, FCS added after sterilization stored at $4^{\circ} \mathrm{C}$ and used sterile.

Flow Comp ${ }^{\mathrm{TM}}$ isolation buffer

$\begin{array}{lll}\text { FBS } & 2 \%(\mathrm{v} / \mathrm{v}) & \text { PAA } \\ \text { EDTA } & 1 \mathrm{mM} & \text { Invitrogen }\end{array}$

In sterile D-PBS. Stored at $4^{\circ} \mathrm{C}$ and used sterile.

\subsubsection{Buffers for flow cytometry}

Annexin-V binding buffer

$\begin{array}{lll}\text { HEPES } & 10 \mathrm{mM} & \text { Sigma-Aldrich } \\ \mathrm{NaCl} & 140 \mathrm{mM} & \text { BDH } \\ \mathrm{CaCl}_{2} & 2.5 \mathrm{mM} & \text { BDH }\end{array}$

In D-PBS (in-house), $\mathrm{pH}$ adjusted to 7.4. Stored at $4^{\circ} \mathrm{C}$, used within one month of preparation.

\section{FACS buffer}

Bovine serums albumin (BSA) $\quad 0.1 \%(\mathrm{w} / \mathrm{v}) \quad$ Invitrogen

Sodium azide

$0.01 \%(\mathrm{v} / \mathrm{v}) \quad$ Sigma-Aldrich

In D-PBS (in-house). Stored at $4^{\circ} \mathrm{C}$.

\subsubsection{Solutions for preparation of clodronate liposomes}

\section{Cholesterol stock solution}

Cholesterol

$20 \mathrm{mg} / \mathrm{mL}$

Sigma-Aldrich

In chloroform (Merck). Stored at $-20^{\circ} \mathrm{C}$. 
Dichloromethlene diphophonate $\left(\mathrm{Cl}_{2} \mathrm{MDP}\right)$ solution

$\mathrm{Cl}_{2} \mathrm{MDP}$

$0.7 \mathrm{M}$

Sigma-Aldrich

In MilliQ water, $\mathrm{pH}$ adjusted to 7.1 . Stored at $-20^{\circ} \mathrm{C}$.

Phosphatidylcholine stock solution

L- $\alpha$-phosphatidylcholine

$100 \mathrm{mg} / \mathrm{mL}$

Sigma-Aldrich

In chloroform (Merck). Stored at $-20^{\circ} \mathrm{C}$.

\subsubsection{Solutions for immunohistochemistry}

Blocking buffer

$\begin{array}{lll}\text { Saponin } & 0.1 \%(\mathrm{w} / \mathrm{v}) & \text { Sigma-Aldrich } \\ \text { BSA } & 0.1 \%(\mathrm{w} / \mathrm{v}) & \text { Invitrogen } \\ \text { Sodium azide } & 0.01 \%(\mathrm{v} / \mathrm{v}) & \text { Sigma-Aldrich } \\ \text { FBS } & 10 \%(\mathrm{v} / \mathrm{v}) & \text { Sigma-Aldrich, } \\ & & \text { Invitrogen }\end{array}$

In D-PBS (in-house). Stored at $4^{\circ} \mathrm{C}$.

\subsubsection{Buffers for protein or antibody labelling}

Sodium bicarbonate $\left(\mathrm{NaHCO}_{3}\right.$ buffer)

$\mathrm{NaHCO}_{3} \quad 0.1 \mathrm{M} \quad$ Sigma-Aldrich

In MilliQ water. Prepared fresh before each use.

Tris buffer

Tris $0.1 \mathrm{M} \quad$ Invitrogen

In MilliQ water, $\mathrm{pH}$ adjusted to 8.5. Stored at RT.

\subsection{Proteins and copolymers}

Glatiramer Acetate (GA, Copaxone $\left.{ }^{\circledR}\right)$

Purchased from Teva pharmaceutical Industries Ltd. (Petach Tikva, Israel). 


\section{$2.4 \quad$ Kits}

Biotin/Avidin blocking kit

Bioplex bead array kit (IL-6, KC, MCP-1)

Diff-Quik kit

IL-1 $\beta$ ELISA kit

Limulus amebocyte lysate kit

Murine GM-CSF ELISA kit

Murine M-CSF ELISA kit

$\mathrm{TNF} \alpha$ ELISA kit
Invitrogen

Bio-Rad,

Hercules, CA, USA

Dade Behring,

Newark, USA

R\&D Systems,

Minneapolis, USA

Associates of

Cape Cod, Inc.,

Falmouth, MA, USA

PeproTech Inc.,

Rocky Hill, NJ, USA

PeproTech Inc.,

Rocky Hill, NJ, USA

BD Bioscience,

San Diego, CA, USA 
Table 2.1: Antibodies used for flow cytometry and immunofluorescent histology.

All antibodies for use in flow cytometry or immunofluorescent histology were titrated before use to determine the ideal concentration for use.

\begin{tabular}{|c|c|c|c|c|}
\hline Antigen & Clone & Source & Isotype & Fluorophore \\
\hline Annexin-V & & Pharmingen & & FITC \\
\hline $\mathrm{CD}^{\mathrm{a}}$ & $2 \mathrm{C} 11$ & eBioscience & Hamster IgG & SA-APC ${ }^{b}$ \\
\hline $\mathrm{CD} 28^{\mathrm{a}}$ & 37.51 & homemade & Hamster IgG & \\
\hline CD4 & RM4-5 & Pharmingen & Rat IgG2a, к & PerCP \\
\hline $\mathrm{CD} 8^{\mathrm{a}}$ & 2.43 & homemade & Rat IgG2b, $\kappa$ & $\mathrm{PE}$ \\
\hline CD11b & $\mathrm{M} 1 / 70$ & $\begin{array}{l}\text { Pharmingen, } \\
\text { eBioscience, } \\
\text { BioLegend }\end{array}$ & Rat IgG2b, $\kappa$ & $\begin{array}{l}\text { APC, FITC, PE, } \\
\text { PerCP-Cy5.5 }\end{array}$ \\
\hline $\mathrm{CD} 14^{\mathrm{c}}$ & $\begin{array}{l}\mathrm{Sa} 2-8 \\
\text { (unlabelled) } \\
\text { Sa14-2 }\end{array}$ & $\begin{array}{l}\text { eBioscience } \\
\text { BioLegend }\end{array}$ & Rat IgG2a, $\kappa$ & $\begin{array}{l}\text { PerCP-Cy5.5 } \\
\text { arat-Alexa555 } \\
\text { arat-Alexa488 }^{\mathrm{c}}\end{array}$ \\
\hline CD206 & MR5D3 & SeroTec & Rat IgG2a & $\mathrm{PE}$ \\
\hline Cytokeratin & $\begin{array}{l}\text { Polyclonal } \\
\text { (unlabelled) }\end{array}$ & abcam & Rabbit IgG & $\begin{array}{l}\text { arabbit-FITC }^{\mathrm{c}} \\
\text { arabbit-Alexa594c }^{\mathrm{c}}\end{array}$ \\
\hline F4/80 & $\begin{array}{l}\text { BM8 } \\
\text { CI:A3-1 }\end{array}$ & eBioscience & Rat IgG2a, $\kappa$ & PE, PerCP \\
\hline $\mathrm{FcR}^{\mathrm{a}}$ & 24G.2 & homemade & Rat IgG2b, к & \\
\hline Gr-1 & RB6-8C5 & Pharmingen & Rat IgG2b, $\kappa$ & APC \\
\hline Ly-6G & $1 \mathrm{~A} 8$ & Pharmingen & Rat IgG2a, $\kappa$ & $\mathrm{PE}$ \\
\hline MCP-1 & $4 \mathrm{E} 2$ & eBioscience & $\begin{array}{l}\text { Hamster } \\
\operatorname{IgG} 1, \kappa\end{array}$ & FITC $^{\mathrm{c}}$ \\
\hline Neutrophils & $7 / 4$ & SeroTec & IgG2a & FITC \\
\hline PI & & Pharmingen & & \\
\hline
\end{tabular}




\subsection{Preparation of monosodium urate crystals}

A supersaturated solution of uric acid (Sigma-Aldrich), adjusted to mildly basic conditions, was prepared to allow for the crystallization of MSU crystals. Briefly, 250mg uric acid was added to $45 \mathrm{~mL}$ of MilliQ water containing $300 \mu \mathrm{L}$ of $5 \mathrm{M} \mathrm{NaOH}$ (Sigma-Aldrich) and the solution was boiled until the uric acid dissolved. The solution was filter sterilized using $0.22 \mu \mathrm{m}$ vacuum driven disposable bottle top filters, following which, $1 \mathrm{~mL}$ of $5 \mathrm{M}$ $\mathrm{NaCl}(\mathrm{BDH})$ was added to the hot solution, and the solution stored at $26^{\circ} \mathrm{C}$ to allow crystallization. After seven days the MSU crystals were washed with ethanol (BDH) and acetone $(\mathrm{BDH})$ over a buchner flask then placed under sterile conditions and allowed to air dry. Physical characteristics typically displayed by MSU crystals include, a needle-like shape, lengths between $5-20 \mu \mathrm{m}$ and displaying optical birefringence under the microscope. MSU crystals were confirmed as endotoxin-free by LAL assay $(<0.01$ $\mathrm{EU} / 10 \mathrm{mg})$.

\subsection{Endotoxin levels}

As endotoxin can activate innate immune cells, reagents and glassware used were tested to ensure the cell cultures were not only sterile but also free from endotoxins. All plasticware were explicitly pyrogen (endotoxin)-free. Reagents used were either certified as low endotoxin or tested by LAL assay for endotoxin levels.

\subsubsection{Limulus amebocyte lysate (LAL) assay test}

The following were tested for endotoxin levels by LAL assay kit. The kit was run according to manufacturer's instructions.

MSU crystals

MilliQ water

Cleaned and autoclaved glassware
$<0.01 \mathrm{EU} / 10 \mathrm{mg}$

$<0.01 \mathrm{EU} / \mathrm{mL}$

$<0.01 \mathrm{EU} / \mathrm{mL}$

(The inside of a $250 \mathrm{~mL}$ piece of glassware was rinsed with $10 \mathrm{~mL}$ of water provided in the LAL kit and tested). 


\subsubsection{Purchased reagents}

$\begin{array}{lll}5 \mathrm{M} \mathrm{NaCl} & \text { Sigma-Aldrich } & <0.3 \mathrm{EU} / \mathrm{mL} \\ \text { D-PBS } & \text { Invitrogen } & <0.03 \mathrm{EU} / \mathrm{mL} \\ \text { RPMI-1640 } & \text { Invitrogen } & <0.03 \mathrm{EU} / \mathrm{mL} \\ \text { Puregene RBC lysis buffer } & \text { QIAGEN, } & <0.03 \mathrm{EU} / \mathrm{mL} \\ & \text { Valencia, CA, USA } & \\ \text { Fetal bovine serum } & \text { Invitrogen } & <0.08 \mathrm{EU} / \mathrm{mL} \\ & \text { Sigma-Aldrich } & <0.13 \mathrm{EU} / \mathrm{mL}\end{array}$

\subsection{Animal studies}

\subsubsection{Maintenance and ethic approvals}

All mice were bred and housed at the Biomedical Research Unit of the Malaghan Institute of Medical Research, based at Victoria University of Wellington, New Zealand. All experimental procedures were approved by the Victoria University Animal Ethics Committee in accordance with their guidelines, under the license codes 2005R7M (Inflammatory immune profiling of neutrophil mouse models of inflammation) or 2006R19 (The use of mouse tissues for multiple research projects).

\subsubsection{Mice}

C57Bl/6J mice were originally purchased from Jackson Laboratory (Bar Harbour, ME, USA). All animals used for experiments were male, aged between $8-11$ weeks.

\subsubsection{Murine peritoneal model of MSU-induced inflammation}

Mice were administered with an intraperitoneal (i.p.) injection of $3 \mathrm{mg}$ MSU crystals suspended in $0.5 \mathrm{~mL}$ D-PBS. At different time-points, mice were euthanased by carbon dioxide asphyxiation followed by cervical dislocation. 
Blood was then harvested by cardiac puncture into sterile $10 \mathrm{~mL}$ tubes (SARSTEDT, Australia Pty Ltd., Australia) containing $600 \mu \mathrm{L}$ volume of $125 \mathrm{U} / \mathrm{mL}$ heparin (Wellington Hospital, NZ) in D-PBS stored on ice.

Peritoneal leukocytes were harvested by peritoneal lavage. Mice were dowsed with 70\% ethanol and the skin covering the peritoneum carefully removed without disturbing the peritoneal membrane. D-PBS $(3 \mathrm{~mL})$ containing $25 \mathrm{U} / \mathrm{mL}$ heparin was injected into the peritoneal cavity. The inguinal fat pads were used as sites of injection to allow the injection hole to re-seal upon extraction of syringe needle. The peritoneum was then massaged for 20 seconds following which the heparin/D-PBS (containing peritoneal exudates cells) was extracted using the same syringe used for injection. The peritoneal lavage fluid was kept in the syringe to maintain sterility and then placed immediately on ice.

\subsubsection{Fluorescent staining of murine $\mathrm{CD}_{11} \mathrm{~b}^{+}$blood cells in vivo}

C57Bl/6J mice were administered with a $200 \mu \mathrm{L}$ i.v. tail injection of $7 \mu \mathrm{g} / \mathrm{mL}$ fluorescentlabelled protein (section 2.3), then left naïve or treated with MSU crystals (section 2.5). At different time-points, mice were euthanased by carbon dioxide asphyxiation followed by cervical dislocation. Blood and peritoneal exudates cells (PECs) were harvested as described for MSU crystal-induced inflammation above.

\subsection{Cell harvest and processing}

\subsubsection{Preparation of cells from peritoneal lavage}

PECs were harvested from naïve or treated mice at different time-points as described in 2.8.3. Under sterile conditions, the peritoneal lavage was expelled into a $15 \mathrm{~mL}$ sterile tube

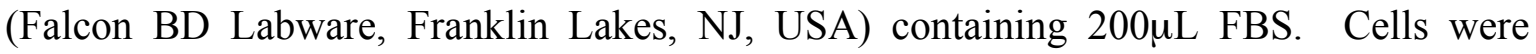
pelleted by centrifugation with a Heraeus Megafuge 2.0R (DJB Labcare, Buckinghamshire, England) for five minutes at $500 \mathrm{xg}$ (RT) and the lavage fluid stored for cytokine/chemokine analysis (section 2.4). Pelleted cells were washed three times in room temperature D-PBS before further culture or staining with fluorescent-labelled antibodies. 
Cytospin samples of the cells were prepared, stained with Diff-Quik (section 2.4) and differential cell counts performed (section 2.12.1). For counting PECs, 10 $\mu \mathrm{L}$ of cell suspension was mixed with $90 \mu \mathrm{L}$ of $0.4 \%$ Trypan Blue Stain (Invitrogen). The mixture was placed on a haemocytometer and the viable cells that excluded the dye were counted under 10x magnification with an Olympus BX40 (Olympus, Central Valley, PA, USA) microscope.

\subsubsection{Preparation of cells from mouse blood}

Blood was collected by cardiac puncture or by tail-bleeding from naïve or treated mice at different time-points. Under sterile conditions, RBCs were pelleted by centrifugation for five minutes at $500 \mathrm{x} \mathrm{g}(\mathrm{RT})$ and the serum stored for cytokine/chemokine analysis (section 2.4). Pelleted cells were gently resuspended in $5-10 \mathrm{~mL}$ of Puregene RBC lysis buffer (QIAGEN, Valencia, CA, USA) and incubated with atmospheric air and $5 \% \mathrm{CO}_{2}$ in a HERAcell 240 tissue culture incubator (Thermo Scientific, Cheshire, UK) for 15 minutes at $37^{\circ} \mathrm{C}$. Cells were then washed twice in D-PBS before further culture or antibody staining for analysis by flow cytometry (section 2.11). If RBC lysis was incomplete, the process was repeated.

\subsubsection{Preparation of cells from spleens and lymph nodes}

Mice from naïve or treated groups were euthanased by carbon dioxide asphyxiation followed by cervical dislocation. Mice were dowsed with 70\% ethanol and the peritoneal cavity was carefully opened. The inguinal, mesenteric lymph nodes or spleens were removed as required. The lymph nodes and spleens were placed in Collagenase II/DNase I solution (section 2.2.1) on ice until further use.

For investigating total cell populations in lymphoid tissues, spleens were injected with 1 $2 \mathrm{~mL}$ of Collagenase II/DNase I solution and the flow-through collected in 24 well plates (Falcon BD Labware, Franklin Lakes, NJ, USA). The spleens were then placed in the collected solution and incubated for 30 minutes at $37^{\circ} \mathrm{C}$. Harvested lymph nodes were placed directly in $1 \mathrm{~mL}$ Collagenase II/DNase I solution in 24 well plates and incubated as 
above. For the isolation of lymphocytes from spleens and lymph nodes, Collagenase II/DNase I digestion was excluded.

Following incubation, lymph nodes and spleens were disrupted with the back of the plunger from $1 \mathrm{~mL}$ syringes (BD, Singapore) and flushed with cRPMI through 40 $\mu$ m nylon cell strainers (Falcon BD Labware, Franklin Lakes, NJ, USA) into 50mL tubes (Falcon BD Labware, Franklin Lakes, NJ, USA). Suspensions were then centrifuged for five minutes at $800 \mathrm{x} \mathrm{g}$ (RT) and contaminating RBCs lysed with Puregene RBC lysis buffer as described (section 2.8.2). Cells were washed once and resuspended in cRPMI, filtered though $40 \mu \mathrm{m}$ cell strainers and placed on ice until further use. Cell counts were performed as described for PECs (section 2.8.1).

\subsubsection{Preparation of primary mouse peritoneal mesothelial cells}

Harvesting and culturing of primary mouse PMCs was performed as previously described $(126,127)$. Naïve $\mathrm{C} 57 \mathrm{Bl} / 6 \mathrm{~J}$ mice were euthanased by carbon dioxide asphyxiation followed by cervical dislocation. In a safety cabinet (class II, type A2) under sterile conditions, mice were sprayed with $70 \%$ ethanol and the skin and fur covering the peritoneum carefully separated without puncturing the peritoneal lining. Resident peritoneal leukocytes were removed by injecting $5 \mathrm{~mL}$ of $25 \mathrm{U} / \mathrm{mL}$ heparin in D-PBS into the peritoneal cavity, injecting near the inguinal fat pads to allow resealing and minimal leakage of the injection site upon needle extraction. After gently massaging the inflated peritoneum, the heparin/D-PBS (containing resident leukocytes) was extracted with a syringe, and the lavage repeated. $5 \mathrm{~mL}$ of $0.25 \%$ Trypsin/EDTA (Invitrogen) pre-warmed to $37^{\circ} \mathrm{C}$ was injected into the peritoneal cavity and allowed to sit for 15 minutes under a heat lamp with occasional massaging. The outside of the peritoneum was kept moist with D-PBS. The Trypsin/EDTA was extracted and transferred to a sterile $50 \mathrm{~mL}$ tube containing $200 \mu \mathrm{L}$ FBS. The peritoneum was then washed twice with cRPMI and the extracted cells expelled into the same $50 \mathrm{~mL}$ collection tube.

The harvested cells were pelleted by centrifugation for five minutes at $500 \mathrm{x} \mathrm{g}\left(20^{\circ} \mathrm{C}\right)$, washed twice in D-PBS. The final cell pellet was resuspended in peritoneal mesothelial cell (PMC) growth media (section 2.2.1) and cultured in $75 \mathrm{~cm}^{2}$ collagen-coated culture 
flasks (Falcon BD Labware, Franklin Lakes, NJ, USA). Cells were grown out to the third passage (section 2.8.5) before use in experiments. Cells were confirmed as mesothelial by a squamous cobble stone appearance and positive immunofluorescence staining (section 2.12.2) with a fluorescent-labelled anti-cytokeratin antibody.

\subsubsection{Culturing peritoneal mesothelial cells}

Primary mouse PMCs were harvested and cultured in $75 \mathrm{~cm}^{2}$ collagen-coated culture flasks (section 2.8.4). Just prior to cell confluence, the media was removed and the cells gently rinsed with D-PBS warmed to $37^{\circ} \mathrm{C}$. $10 \%$ Trypsin/EDTA in D-PBS was added to cover the bottom of the flask, and the solution incubated at $37^{\circ} \mathrm{C}$. After two minutes, cells detachment was inspected using an Olympus CK2 inverted microscope (Olympus, Central Valley, PA, USA). With gentle tapping, mesothelial cells detached from the culture flask while any contaminating macrophages remained adherent. The activity of Trypsin/EDTA was terminated by the addition of cRPMI $(2 \mathrm{~mL})$ to the flask. The harvested mesothelial cells were pelleted from the solution by centrifugation for five minutes at $500 \mathrm{x} g$ (RT) then resuspended in D-PBS and washed twice before further culture or preparation for immunofluorescent histology (section 2.12.2). Cells were counted as described for PECs (section 2.8.1).

\subsection{Cell purification}

\subsubsection{Enrichment of mouse monocytes and neutrophils}

Monocytes were isolated from PECs or peripheral blood from mice treated with MSU as described in sections 2.8.1 and 2.8.2 respectively. Individual samples were pooled in sterile $50 \mathrm{~mL}$ tubes, washed twice in D-PBS and resuspended in $5 \mathrm{~mL}$ D-PBS. $4 \mathrm{~mL}$ of Lympholyte ${ }^{\circledR}-\mathrm{M}$ cell separation media (Cedarlane Laboratories Ltd., Hornby, Canada) was added below the cell suspension using sterile pasteur pipettes (Volac, Bacto Laboratories Pty Ltd., NSW, Australia). The cells were centrifuged for 20 minutes at $1200 \mathrm{x}$ g (RT). Enriched monocytes formed a buffy layer above the gradient medium (Diagram 2.1). Monocytes were collected and transferred to fresh $50 \mathrm{~mL}$ tubes using transfer pipettes (Samco Scientific, San Fernando, CA, USA), washed twice, resuspended in cRPMI and 
placed on ice until further use.. This procedure resulted in a cell preparation that was greater than $75 \%$ monocytes.

Neutrophils were also isolated from PECs using Lympholyte ${ }^{\circledR}-\mathrm{M}$ cell density gradients as described for monocytes above. In contrast to monocytes, the neutrophil-rich fraction pelleted to the bottom of the gradient (Diagram 2.1). The supernatant was carefully removed by decanting, and the cells washed twice in D-PBS, then resuspended in cRPMI and placed on ice until further use. This procedure resulted in a preparation what was greater that $80 \%$ neutrophils.

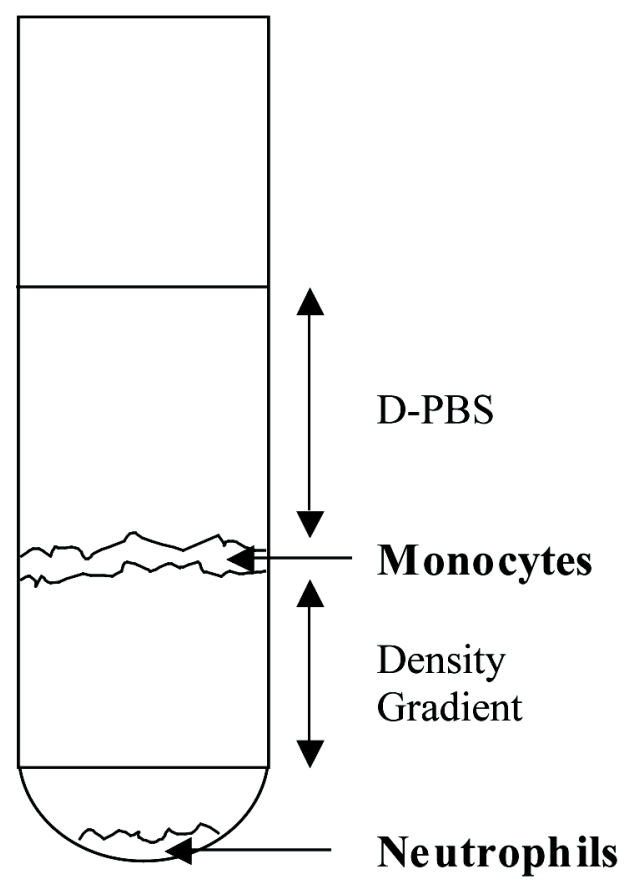

Diagram 2.1: Schematic representation of cell purification by Lympholyte $^{\circledR}-\mathrm{M}$ density gradient. After centrifugation for 20 minutes at $1200 \mathrm{x} g$ (RT) monocytes from blood or peritoneal exudate cells formed a buffy layer directly above the gradient medium. Neutrophils were pelleted at the bottom of the tube.

\subsubsection{Magnetic sorting of $\mathrm{CD}_{11} \mathrm{~b}^{+}$cells}

Monocytes were isolated from the blood using Lympholyte ${ }^{\circledR}-\mathrm{M}$ cell density gradients as described (section 2.9.1). The autoMACS ${ }^{\mathrm{TM}}$ cell separation system (Miltenyi Biotec, Bergisch Gladbach, Germany) was used for $\mathrm{CD}_{11 b^{+}}$cell isolation. The cells were 
resuspended in $2 \mathrm{~mL}$ autoMACS running buffer (section 2.2.2) and filtered through $30 \mu \mathrm{m}$ pre-separation filters (Miltenyi Biotec, Bergisch Gladbach, Germany). To remove dead cells and debris, a positive selection was performed using an autoMACS separator (program "possel", Miltenyi). The negative fraction was collected, washed once and resuspended in $200 \mu \mathrm{L}$ running buffer. PE-conjugated antibody specific for CD11b (Table 2.1) was added to a dilution factor of $1: 2,000 \mu \mathrm{L}$ and the cells incubated for 15 minutes on ice. The cells were then washed twice in $50 \mathrm{~mL}$ running buffer and resuspended in $200 \mu \mathrm{L}$. Anti-PE beads ((Miltenyi Biotec, Bergisch Gladbach, Germany) were added to a dilution factor of $1: 20 \mu \mathrm{L}$, and the cells incubated for 30 minutes at $4{ }^{\circ} \mathrm{C}$ under rotation. Cells were then washed and resuspended in $2 \mathrm{~mL}$ and separated using the autoMACS separator as above. The positive fraction contained the $\mathrm{CD}_{11} \mathrm{~b}^{+}$purified cells $\left(>80 \% \mathrm{CD} 1 \mathrm{~b}^{+}\right)$. The suspensions were then washed once and resuspended in cRPMI for cell culture.

\subsection{In vitro assays}

\subsubsection{Restimulation assay}

Harvested PECs from naïve, MSU- or thioglycollate- treated mice were purified by density gradient (section 2.9.1) and cultured in cRPMI at $5 \times 10^{5}$ cells $/ \mathrm{mL}, 200 \mu \mathrm{L}$ per well in 96 well flat-bottom plates (Falcon BD Labware, Franklin Lakes, NJ, USA). Cells were either left unstimulated, treated with $500 \mu \mathrm{g} / \mathrm{mL}$ MSU crystals, or with $100 \mathrm{ng} / \mathrm{mL}$ Lipopolysccharide, E.coli 0111:B4 (LPS, Biolab Scientific, Australia) and incubated for eight hours at $37^{\circ} \mathrm{C}$. Supernatants were then harvested and stored at $-20^{\circ} \mathrm{C}$ until analysis for cytokines and chemokines (section 2.4).

\subsubsection{Phagocytic assay}

PECs were harvested from naïve or MSU treated mice and purified by density gradient (section 2.9.1). Cells were resuspended in cRMPI at $5 \times 10^{5}$ cells $/ \mathrm{mL}$ and $2 \mathrm{~mL}$ of cell suspension was placed into sterile $10 \mathrm{~mL}$ tubes. FluoSpheres ${ }^{\circledR}$ fluorescent microspheres ( $2 \%$ solids, Invitrogen) were diluted in D-PBS and $50 \mu \mathrm{L}$ of the diluted beads were added to the cells with a final dilution factor of 1:20,000 of the original $2 \%$ solids solution. The 
cell suspensions with added beads were then placed on a rotator (200rpm, $15 \mathrm{~mm}$ radius) and incubated for 30 minutes at $37^{\circ} \mathrm{C}$. FBS at a volume of $1 \mathrm{~mL}$ was then added below the cell suspension using sterile pasteur pipettes and centrifuged for five minutes at $800 \mathrm{xg}$ (RT). This pelleted the cells while separating non-phagocytosed beads at the serummedium interface. The supernatant was then removed and the cells washed twice in FACS buffer, stained for the appropriate cell surface markers and analysed by flow cytometry (section 2.11).

\subsubsection{Proliferation assay}

Lymphocytes or splenocytes were isolated from naïve or treated mice as described (section 2.8.3). Cells were resuspended in cRPMI at $1 \times 10^{6}$ cells $/ \mathrm{mL}$ and $50 \mu \mathrm{L}$ of cell suspension

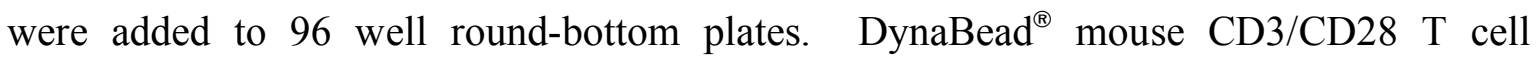
expander beads (Invitrogen) in D-PBS were added to the wells at a final concentration of $2.5 \times 10^{4}$ beads/well. The cultures were then incubated for 20 hours at $37^{\circ} \mathrm{C}$.

Alternatively, antibody-coated plates were used instead of expander beads. To coat 96well plates, anti-mouse CD3 antibody (Table 2.1) and anti-mouse CD28 (Table 2.1) were diluted to $2 \mu \mathrm{g} / \mathrm{mL}$ and $3 \mu \mathrm{g} / \mathrm{mL}$ respectively in D-PBS and incubated in the wells overnight at $37^{\circ} \mathrm{C}$. Lymphocytes were then added as described above and incubated for 20 hours at $37^{\circ} \mathrm{C}$.

After incubation, 25 $\mu \mathrm{L}\left[{ }^{3} \mathrm{H}\right]$-thymidine (GE Healthcare, Buckinghamshire, UK) diluted 1:100 in cRMPI (final concentration $0.5 \mathrm{mCi}$ ) was added to each well and the cultures incubated for a further eight hours. At different time points, cells were harvested from the culture plates onto printed glass fibre filter mats (PerkinElmer Lifesciences Inc., Boston, MA, USA) using a Tomtec automatic 96 well plate harvester (Tomtec, Hamden, CT, USA). Filter mats were dried in a microwave oven, placed into plastic sample bags (PerkinElmer, Lifesciences Inc., Boston, MA, USA) and scintillated with Beta Scint liquid scintillation cocktail (Wallac, Turku, Finland). The sample bags were sealed with a heat sealer (Wallac, Turku, Finland) and $\left[{ }^{3} \mathrm{H}\right]$-thymidine incorporation was analysed using a 1459 Microbeta Plus liquid scintillation counter (Wallac, Turku, Finland). 


\subsubsection{Suppression assay}

Monocytes from PECs or $\mathrm{CD}_{1} 1 \mathrm{~b}^{+}$cells from blood were purified as described (section 2.9.1/2.9.2 respectively). Cells were resuspended in cRPMI at $5 \times 10^{5}$ cells $/ \mathrm{mL}$ and $50 \mu \mathrm{L}$ of serial diluted $(2 \mathrm{x})$ cell suspensions were added to the appropriate wells containing lymphocytes or splenocytes and a proliferation assay carried out as described in section 2.11.3.

\subsubsection{Peritoneal mesothelial cell stimulation assay}

Cultured PMCs (section 2.8.5) were suspended in cRPMI at $1 \times 10^{5}$ cells $/ \mathrm{mL}$ and $200 \mu \mathrm{L}$ of cell suspension was placed into a sterile 96 well flat-bottom plate. Cells were incubated for six hours, after which the culture medium was replaced with fresh cRPMI. Anti-mouse CD14 blocking antibody (Table 2.1) was added to the appropriate wells at a final concentration of $20 \mu \mathrm{g} / \mathrm{mL}$ and incubated for one hour at $37^{\circ} \mathrm{C}$. After incubation, cells were either left unstimulated or treated with $500 \mu \mathrm{g} / \mathrm{mL}$ MSU crystals, or $100 \mathrm{ng} / \mathrm{mL}$ LPS and incubated for six hours at $37^{\circ} \mathrm{C}$. Supernatants were then harvested and stored at $-20^{\circ} \mathrm{C}$ until analysed for cytokine and chemokine production (section 2.4).

\subsubsection{Peritoneal membrane stimulation assay}

Naïve mice were sacrificed by carbon dioxide asphyxiation followed by cervical dislocation. Mice were sprayed with $70 \%$ ethanol and under sterile conditions the skin and fur covering the peritoneum was carefully separated without puncturing the peritoneal lining. The ventral tissue covering the peritoneal cavity was excised in approximately $1.5 \mathrm{~cm}^{2}$ pieces and placed into cRPMI in 24 well plates, on ice. In a 96 well round-bottom plate, with the appropriate wells containing $200 \mu \mathrm{L}$ cRPMI, wells were either left untreated, or added with $500 \mu \mathrm{g} / \mathrm{mL}$ MSU crystals or $100 \mathrm{ng} / \mathrm{mL}$ LPS. The visceral side of the excised peritoneal tissue was placed face down over the top of the wells and then the lid of the 96well plate placed over the top so as to form a seal between the tissue and the well.

Alternatively, for CD14 blocking permutations, a final concentration of $20 \mu \mathrm{g} / \mathrm{mL}$ of antimouse CD14 antibody was added to the wells and incubated with the appropriate peritoneal tissue for one hour at $37^{\circ} \mathrm{C}$ prior to the addition of stimulants. The plate was 
then inverted to allow the media containing stimulants to contact the peritoneal tissue. Tissues were then incubated for six hours at $37^{\circ} \mathrm{C}$, following which the supernatants were collected and assayed for cytokines/chemokines by ELISA and cytokine bead array (section 2.4).

\subsubsection{Preparation of neutrophil secretion}

Neutrophils were harvested from mice 18 hours after MSU administration and purified from PECs using Lympholyte ${ }^{\circledR}-\mathrm{M}$ cell density gradients as described (section 2.9.1). Control neutrophils were cultured in cRPMI to give a high level of neutrophil viability over the period of incubation. Neutrophils were suspended at $1 \times 10^{6}$ cells $/ \mathrm{mL}$ and $2 \mathrm{~mL}$ of cell suspension was placed into each well of sterile 24 well plates. Cells were either left unstimulated or treated with $500 \mu \mathrm{g} / \mathrm{mL}$ MSU crystals and incubated for eight hours at $37^{\circ} \mathrm{C}$. Supernatants were then harvested and stored at $-20^{\circ} \mathrm{C}$ until being assayed for cytokines and chemokines (section 2.4).

To induce apoptosis in neutrophils, neutrophils starved of serum by being cultured in serum-free cRPMI (section 2.2.1). Neutrophils were suspended at $1 \times 10^{6}$ cells $/ \mathrm{mL}$ and $2 \mathrm{~mL}$ of cell suspension was placed into a sterile $50 \mathrm{~mL}$ tube. Cells were then placed on a rotator (200rpm, $15 \mathrm{~mm}$ radius) and incubated for eight hours at $37^{\circ} \mathrm{C}$. Supernatants were harvested and stored at $-20^{\circ} \mathrm{C}$ until being assayed for cytokines and chemokines (section 2.4). The percentage of apoptotic neutrophils was determined by annexin-V/PI double staining (section 2.14.1) together with the appropriate fluorescent-labelled antibodies (Table 2.1) and analysed by flow cytometry (section2.12).

\subsubsection{Culturing of monocytes with neutrophil secretions}

Mixed PECs or purified monocyte populations $\left(5 \times 10^{5}\right.$ cells $/ \mathrm{mL}, 200 \mu \mathrm{L}$ per well) were suspended in either fresh cRPMI, cRPMI containing secretions from viable neutrophils (section 2.10.7), RPMI containing secretions from apoptotic neutrophils (section 2.10.7) with $10 \%$ FBS added to the monocyte culture (v/v), or untreated neutrophil secretions. Cells were either left unstimulated, treated with $500 \mu \mathrm{g} / \mathrm{mL}$ MSU crystals, or with 
$100 \mathrm{ng} / \mathrm{mL}$ LPS and incubated for eight hours at $37^{\circ} \mathrm{C}$. Supernatants were then harvested and stored at $-20^{\circ} \mathrm{C}$ until analysis for cytokines and chemokines (section 2.4).

\section{$2.11 \quad$ Flow cytometry}

Cell suspensions from various preparations were transferred in a volume of $200 \mu \mathrm{L}$ to a 96 well round-bottom plate. The cells were centrifuged for one minute at $1800 \times \mathrm{g}(\mathrm{RT})$, the supernatant flicked off and the cells resuspended and washed again in $200 \mu \mathrm{L}$ FACS buffer. The cells were incubated with anti-mouse FcR antibody (Table 2.1) in 50 $\mu \mathrm{L}$ FACS buffer for 10 minutes at RT. After incubation, cells were washed in FACS buffer, and subsequently incubated with the appropriate $50 \mu \mathrm{L}$ master mix of fluorophore-labelled antibodies (Table 2.1) for 10 minutes on ice. Cells were then washed in $200 \mu \mathrm{L}$ FACS buffer, filtered through gauze and transferred to Titertube ${ }^{\circledR}$ micro tubes (Bio-Rad, Hercules, CA, USA) .

Flow cytometric analysis was performed on a BD FACSort (BD, San Jose, CA, USA) or BD FACSCalibur (BD, San Jose, CA, USA). Unstained samples were used to distinguish between debris and living cells by their forward scatter (FSC) and side scatter (SSC) properties. Unstained samples were also used to adjust the voltage corresponding to all four channels and samples stained with single fluorescent conjugated antibodies were used to compensate for overflow of fluorescence into other channels. Data acquisition was carried out using the CellQuest ${ }^{\mathrm{TM}}$ Pro software (BD, San Jose, CA, USA) and the data analysed using the FlowJo software (tree Star Inc., Ashland, OR, USA).

\subsection{Histology}

\subsubsection{Morphological histology for differential cell counts}

Cell samples in a volume of $100 \mu \mathrm{L}\left(1 \times 10^{6}\right.$ cells $\left./ \mathrm{mL}\right)$ were centrifuged for five minutes at 800rpm (RT) onto glass slides (LabServ, Auckland, NZ) using a Shandon Cytospin 4 cytocentrifuge (Thermo Scientific, Cheshire, UK) and were allowed to air-dry. Cells were then fixed for five seconds in Diff-Quik Fixative $(1.8 \mathrm{mg} / \mathrm{mL}$ Triarylmethane dye methyl 
alcohol), stained for ten seconds in Diff-Quik Solution I (1g/L Xanthine dye) and then stained for seven seconds in Diff-Quik Solution II (0.625g/L Azure A, 0.625g/L Methylene blue). For differential cell counts, 200 cells were counted per sample.

\subsubsection{Immunofluorescent histology}

Immunoflourescent histology was performed as previously described (128). In brief, resident macrophages or PMCs (section 2.9.5) from naïve mice were suspended in cRPMI at $1 \times 10^{5}$ cell $/ \mathrm{mL}$ and $200 \mu \mathrm{L}$ placed in 8 chamber tissue culture glass slides (Falcon BD Labware, Franklin Lakes, NJ, USA). After incubation for one hour at $37^{\circ} \mathrm{C}$, GolgiStop (BD Biosciences, Mississauga, On, Canada) was added to the appropriate wells at a dilution factor of 1:1500. Cells were then either left unstimulated, treated with $500 \mu \mathrm{g} / \mathrm{mL}$ MSU crystals, or $100 \mathrm{ng} / \mathrm{mL}$ LPS and incubated for four hours at $37^{\circ} \mathrm{C}$.

Following incubation, supernatants were removed and cells fixed and permeabilized with $200 \mu \mathrm{L}$ ice-cold methanol and placed into the freezer at $-20^{\circ} \mathrm{C}$. After ten minutes, cells were washed once with in-house D-PBS (section 2.2.1). Non-specific antibody binding sites were blocked by incubation with block buffer (section 2.2.5) for 30 minutes at $37^{\circ} \mathrm{C}$. After incubation, chambers were washed once with D-PBS and the primary antibodies anti-mouse cytokeratin (Table 2.1), anti-mouse MCP-1 (Table 2.1) or anti-mouse CD14A555 (section 2.14) suspended in block buffer were added to the appropriate wells and incubated at $37^{\circ} \mathrm{C}$. After one hour, chambers were washed three times with D-PBS, and fluorophore conjugated secondary antibodies suspended in block buffer were added to the appropriate wells and incubated for 30 minutes at $37^{\circ} \mathrm{C}$. Cells were then washed three times and fixed with $4 \%$ formalin for ten minutes at RT. Cells were washed three times, the chambers removed, a drop of ProLong Gold anti-fade with DAPI (Invitrogen) applied and the slide mounted with a cover slip (Paul Marienfeld GmbH \& Co., LaudaKönigshofen, Germany). Slides were examined using an Olympus BX51 fluorescent microscope (Olympus, Central Valley, PA, USA) and images visualized using software as described (section 2.16). 


\subsection{Preparation of clodronate liposomes}

Preparation of clodronate liposomes was performed as previously described (129). In brief, $1.51 \mathrm{~mL}$ of phosphatidylcholine stock solution (section 2.2 .3 ) was added to $0.7 \mathrm{~mL}$ cholesterol stock solution (section 2.2.3) in a 500mL round bottom flask. The solvent was removed by placing the flask onto a rotary evaporator at $120 \mathrm{mbar}$ minimum (Vacuum controller V-850, Büchi) and 150rpm rotation (Rotavapor R-210, Büchi Labortechnik AG, Flawil, Switzerland). A thin milky-white phospholipid film formed against the inside of the flask. $17.5 \mathrm{~mL}$ of $0.7 \mathrm{M}$ clodronate solution (section 2.2.3) was added and then the film dispersed by gentle rotation (150rpm) at RT for 10 minutes. The resulting suspension was held under nitrogen gas for two hours at RT and then gently shaken and sonicated in a waterbath at 40kHz (Ultrasonic cleaner FXP8M, Unisonics, Manly Vale, NSW, Australia) for three minutes. The suspension was held under nitrogen gas overnight at $4^{\circ} \mathrm{C}$.

The liposome suspension was transferred to a $50 \mathrm{~mL}$ tube and centrifuged for 40 minutes at $1800 \times \mathrm{g}\left(10^{\circ} \mathrm{C}\right)$ with the brakes off. Liposome-encapsulated clodronate $(1-2 \%$ of the clodronate is encapsulated (129)) formed a white band at the top of the suspension. The non-encapsulated clodronate solution, which sat under the white band of clodronate liposomes, was carefully removed with a syringe. The clodronate solution was filtered through a $32 \mathrm{~mm}$ syringe filter with $0.2 \mu \mathrm{m}$ Supor ${ }^{\circledR}$ membrane and stored at $-20^{\circ} \mathrm{C}$ for repeated use (up to three times). The clodronate liposomes were then washed twice with $10 \mathrm{~mL}$ D-PBS $\left(20\right.$ minutes at $\left.1200 \mathrm{xg}, 10^{\circ} \mathrm{C}\right)$ and resuspended to a final volume of $7 \mathrm{~mL} \mathrm{D}$ PBS. Liposomes were stored at $4^{\circ} \mathrm{C}$ and used within three days of preparation.

\subsubsection{In vivo depletion of resident macrophages}

Mice were either left untreated or injected i.p. with $200 \mu \mathrm{L}$ clodronate liposomes (section 2.13). Three days following the first injection, both untreated and treated mice were challenged with an i.p. injection of $3 \mathrm{mgMSU}$ crystals suspended in $0.5 \mathrm{~mL}$ D-PBS following which PECs were harvested by lavage (section 2.8.1). Immediately before transfer to syringes and before injections, the clodronate liposome suspension was gently shaken to ensure even dispersion. 


\subsection{Fluorophore labelling of antibodies and proteins}

AlexaFluor-488 (Invitrogen), -555 (Invitrogen) or -647 (Invitrogen) were dissolved in Dimethlsulfoxide (DMSO, Sigma-Aldrich) at $20 \mathrm{mg} / \mathrm{mL}$, and stored at $-20^{\circ} \mathrm{C}$ in $10 \mu \mathrm{L}$ aliquots. Proteins (section 2.3 ) at $0.5 \mathrm{mg} / \mathrm{mL}$ or antibodies (Table 2.1) at $1 \mathrm{mg} / \mathrm{mL}$ were resuspended in freshly made $0.1 \mathrm{M} \mathrm{NaHCO}$ buffer (section 2.2.6), placed into Vivaspin 500 exclusion columns (Sartorius, AG, Göttingen, Germany) and centrifuged using a IEC Micromax microcentrifuge (Thermo Scientific, Waltham, MA, USA) at 10,000 rpm for ten minutes, this process was then repeated. Proteins or antibodies were transferred to $1.5 \mathrm{~mL}$ eppendorf tubes (Axygen, Union City, CA, USA) and resuspended to $1.5 \mathrm{mg} / \mathrm{mL}$ in $0.1 \mathrm{M}$ $\mathrm{NaHCO}_{3}$ buffer. While vortexing, $10 \mu \mathrm{g}$ of fluorescent dye was added per $50 \mu \mathrm{g}$ of protein or $100 \mu \mathrm{g}$ of antibody. The samples were then incubated for ten minutes at RT, transferred to Vivapsin 500 exclusion columns and washed with $0.1 \mathrm{M}$ Tris buffer (section 2.2.6) at $10,000 \mathrm{rpm}$ for ten minutes. Samples were then washed twice with D-PBS until the flowthrough became transparent. Labelling of proteins or antibodies was validated by flow cytometry.

\subsubsection{Annexin-V and propidium iodide (PI) double staining}

Purified neutrophils (section 2.9) were washed and stained with FITC-labelled annexin-V $(5 \mu \mathrm{L} / 100 \mu \mathrm{L}$ cells) and $10 \mu \mathrm{g} / \mathrm{mL}$ PI in annexin-V binding buffer (section 2.2.3). Staining was then determined by flow cytometry. Double negatives indicate viable cells, annexin-V single positive indicate early apoptotic cells. PI single positives are necrotic cells, while annexin-V/PI double positives are late stage dying and dead cells.

\subsection{Image analysis and editing}

Microscope images of cells were acquired and optimised using AnalySIS Life Imaging Software (Olympus Soft Imaging Solutions GmbH, Münster, Germany). Photoshop software (Adobe Systems Inc., San Jose, CA, USA) was used on microscopic images to remove debris from acquired images. 


\subsection{Statistical analysis}

To determine statistical significance when there were two treatment groups within an experiment, the Student's T-test was used. The one-way ANOVA and Tukey multiple comparison tests were used in experiments for which there were two or more groups. Statistical significance was determined when $P<0.05$. All statistical analysis were carried out with the software GraphPad Prism (GraphPad Software Inc., San Diego, CA, USA). 
Chapter 3.0:

The Impact of the

Inflammatory Environment on the Differentiation and Function of Recruited Monocytes in MSU-induced Inflammation 


\subsection{Introduction}

A key characteristic of mononuclear phagocytes is their plasticity, making it a challenge to clearly identify myeloid lineage-defined cell subsets. It is becoming increasingly clear that microenvironmental signals can influence the heterogeneity of monocyte-macrophage subsets. Examples of how the local inflammatory environment is capable of altering the monocyte/macrophage phenotype can be observed in a variety of disease conditions such as tumours, autoimmunity and acute inflammation.

\subsubsection{The inflammatory environment in tumours}

During inflammation, mononuclear precursors are recruited into a variety of local environments that influence cellular differentiation and function. For example, antiinflammatory molecules (such as glucocorticoid hormones, IL-4, IL-13 and IL-10) present within the tumour environment generate a population of tumour-associated macrophages (TAMs) $(57,95,96)$. These resident cells are responsible for the production of antiinflammatory cytokines TGF- $\beta$ (98) and IL-10 (97) and contribute to over-suppression of the immune response, leading to tumour angiogenesis (98).

Both the anti-inflammatory cytokine IL-10 released by resident TAMs and macrophagecolony stimulating factor (M-CSF) present in the tumour environment play a prominent role in driving the differentiation of recruited monocyte precursors into TAMs by blocking their differentiation into DCs $(96,108)$. This reprogramming of monocyte differentiation is thought to be an important determinant of the proportion of TAMs versus tumourassociated DCs (TADCs) and their relative distribution in different tumours (130).

Colony-stimulating factors (such as M-CSF) generated during tumour growth are also thought to play a role in the development of an immature myeloid cell population or myeloid-derived suppressor cells (MDSCs) $(96,131,132)$. MDSCs are potent suppressors of $\mathrm{T}$-cell responses, especially $\mathrm{CD}^{+} \mathrm{T}$ cells, and have been associated with tumour expansion in both tumour-bearing mice and cancer patients (133-135). 


\subsubsection{The inflammatory environment in multiple sclerosis}

Multiple sclerosis (MS) is an inflammatory autoimmune disease of the central nervous system (CNS). Experimental autoimmune encephalomyelitis (EAE) is a murine model of this disease extensively used in research. In the CNS of EAE mice, CD $11 b^{+}$Ly $_{6} \mathrm{C}^{\text {hi }}$ myeloid precursor cells (136) are recruited into a local environment rich in IFN- $\gamma$, TNF $\alpha$ (99) and IL-17 $(100,101)$. In these surroundings, recruited monocytes differentiate into macrophages (102) that can escalate disease symptoms through the production of reactive oxygen species and pro-inflammatory cytokines (103), leading to demyelination and axonal damage (104). The same subset of circulating myeloid precursor cells identified in the EAE model have also be found in the spleen and have been shown to suppress CD4 ${ }^{+} \mathrm{T}$ cell proliferation ex vivo (137). These myeloid cells with altered functions have been characterised as MDSCs $(119,137,138)$ and may have displayed differential functions simply because of the differences in recruitment environments (between the CNS and spleen in the EAE model).

\subsubsection{Monocyte recruitment in an MSU-induced inflammatory environment}

Gout is characterized by the deposition of needle-like MSU crystals within the joint synovium $(139,140)$. Using the murine model of gout, recent findings by other members of our group have shown that the initiation of MSU-induced acute inflammation is mediated by activated resident macrophages (58). The early inflammatory profile of gout involves a peak of pro-inflammatory cytokines (predominantly IL-1 $\beta$, IL-6, and TNF $\alpha$ ) within the local environment, which resolves within $4-6$ hours (58). Chemoattractants (such as MCP-1 and KC) have been shown to be responsible for the rapid influx of leukocytes into the peritoneum, peaking at approximately $18-24$ hours after stimulation.

Unlike tumour or EAE models, in MSU-induced inflammation circulating monocytes are recruited into a highly pro-inflammatory environment (IL-1 $\beta$, IL- 6 and TNF $\alpha$ ). Previous studies have proposed that following infiltration, MSU-recruited monocytes play a proinflammatory role through the production of cytokines such as IL- 6 and TNF $\alpha$ (40, 41, $43)$, and then become non-inflammatory as they differentiate into macrophages $(111,113)$. However, the results of these in vitro and ex vivo studies were based on immortalised cells 
expressing monocyte- and macrophage-like phenotypes. Recent ex vivo studies within our group indicate that MSU-recruited monocytes are non-responsive to MSU crystal restimulation and only become pro-inflammatory once they have acquired more macrophage-like characteristics (58). This indicates a different monocyte/macrophage phenotype generated in an acute inflammatory environment, which has not been previously investigated in vivo.

\subsubsection{The inflammatory monocyte subpopulation}

In $\mathrm{C} 57 \mathrm{~B} 1 / 6 \mathrm{~J}$ mice, circulating monocytes are primarily identified by their expression of $\mathrm{CD} 11 \mathrm{~b}$, low levels of F4/80, and intermediate levels of Gr-1 (Ly6C $\mathrm{C}^{-}$and $\mathrm{Ly}_{6 \mathrm{G}}^{+}$) (57). The Gr1-1 ${ }^{\text {hi }}$ circulating monocytes, displaying a $\mathrm{CX}_{3} \mathrm{CR} 1^{\text {low }} \mathrm{CCR} 2^{+}$phenotype, are recruited to inflamed peripheral tissue by MCP-1, the ligand for CCR2 $(57,90,91)$. Consequently, these monocytes are more commonly referred to as inflammatory monocytes (90). Recent findings have shown that these particular subpopulations of circulating monocytes display a tendency to "home" to sites of inflammation and differentiate into F4/80 hi macrophages $(57,141)$.

\subsubsection{Effects of neutrophils on the inflammatory environment}

As previously described, infiltrating neutrophils appear to contribute to the acute proinflammatory environment through the production of IL-8, superoxide and S100A8/A9 proteins $(67,87,103)$. This has the effect of recruiting more neutrophils and amplifies the overall inflammatory response. Neutrophils may also have a direct effect on the monocyte/macrophage phenotype. The phagocytosis of apoptotic neutrophils has been shown previously to drive monocyte/macrophage switching from a "pro"- to "anti"inflammatory phenotype $(113,142,143)$. This process is a common mechanism in the resolution of acute inflammation and may also serve to limit the degree of tissue damage.

\subsubsection{Effects of resident macrophage depletion on cellular recruitment}

Previous studies within our group have shown that in a macrophage-depleted environment, monocyte recruitment is unaffected, whilst neutrophil infiltration is significantly abrogated 
(58). Using MSU crystal stimulation as an in vivo method of monocyte recruitment, this provides an opportunity to examine the impact of a less pro-inflammatory environment (in the absence of resident macrophages and infiltrating neutrophils) on the differentiation and function of MSU-recruited monocytes.

\subsubsection{Objectives}

The aim of this chapter was to determine the effect of different acute inflammatory environments on MSU-recruited monocyte differentiation and function, using a mouse model of MSU-induced peritoneal inflammation. To achieve this, the differentiation pathway of MSU-recruited monocytes in normal $\mathrm{C} 57 \mathrm{Bl} / 6 \mathrm{~J}$ mice was profiled, and compared to the differentiation pathway in macrophage-depleted mice. In addition, the functional phenotype of MSU-recruited monocytes from normal and macrophage-depleted mice was compared during MSU-induced inflammation.

\subsection{Results}

\subsubsection{Profiling the cell subsets observed in MSU-induced inflammation}

To profile the recruited leukocytes involved in MSU-induced inflammation, methods were established to distinguish the different cell populations morphologically. Peritoneal lavages were carried out on naïve or MSU-treated mice and the cells centrifuged onto glass slides, fixed with a DiffQuik staining kit and examined under a microscope. Compared to the resident macrophages from naïve mice (Figure 3.1A), MSU-recruited monocytes were distinguishable by their smaller, less foamy, bean shaped nuclei (Figure 3.2B). While infiltrating neutrophils contained 'multi-lobular'-like nuclei typical of polymorphonuclear cells (Figure 3.1B). 

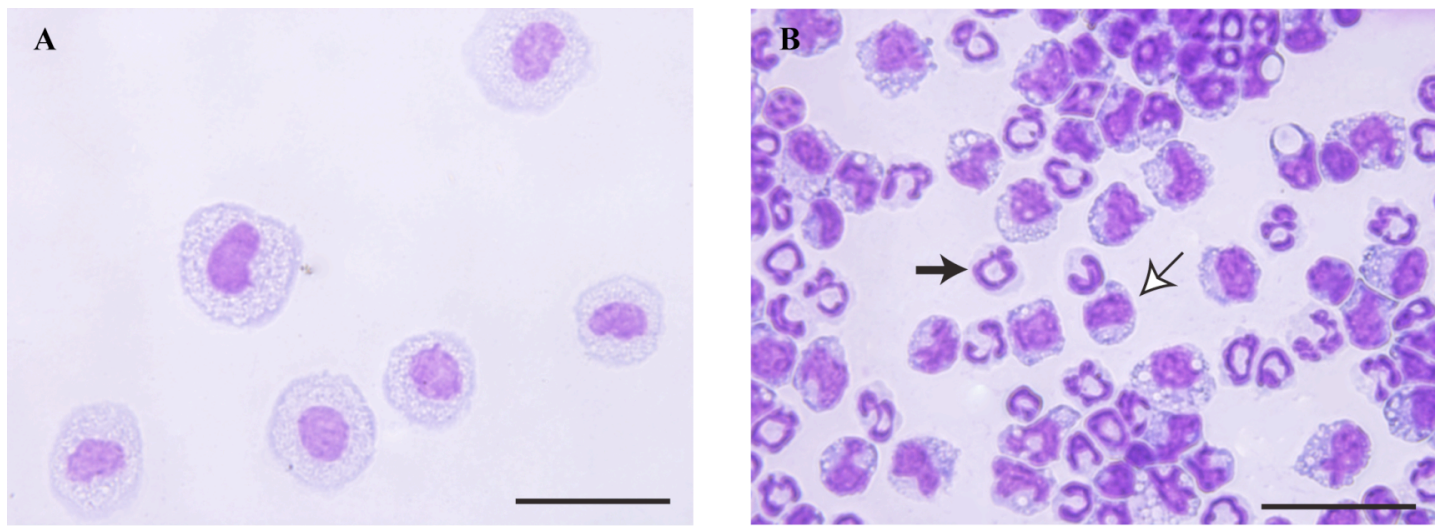

\section{Figure 3.1: Morphology of cells involved in the peritoneal model of MSU-induced}

inflammation. C57B1/6J mice were administered with an i.p. injection of MSU crystals $(3 \mathrm{mg} / 0.5 \mathrm{~mL})$. After 18 hours, peritoneal exudate cells were harvested by lavage. Cells were resuspended in cRMPI 100ul of cell suspension centrifuged onto glass slides using a cytocentrifuge. Cells were then fixed with a DiffQuik staining kit and examined under a microscope. A. Resident macrophages B. MSU-recruited monocytes (white arrow) and neutrophils (black arrow). Original magnification 100x. Scale bar represents 50um. Results are representative of three independent experiments.

Although cell morphology showed an obvious distinction between newly recruited monocytes and fully mature macrophages, clear identification of phenotypic changes at different stages of cell differentiation can be difficult. Therefore, further examination of cell surface markers using flow cytometry was also used in order to distinguish between different cell populations, and more accurately identify the different stages of monocyte to macrophage differentiation in MSU-induced inflammation.

To develop a flow cytometry gating strategy to identify different monocytes/macrophage populations, $\mathrm{C} 57 \mathrm{Bl} / 6 \mathrm{~J}$ mice were administered with an i.p. injection of MSU crystals $(3 \mathrm{mg} / 0.5 \mathrm{~mL})$. After four hours, PECs were harvested and stained for the murine macrophage marker F4/80 and the myeloid antigens Gr-1 and 7/4. Monocyte and neutrophil populations could then be distinguished from each other based on their relative expression of Gr-1 and 7/4, with monocytes expressing Gr- $1^{\text {int }}, 7 / 4^{\text {hi }}$ (Figure 3.2A, R1) and neutrophils displaying Gr-1 $1^{\mathrm{hi}}, 7 / 4^{\mathrm{hi}}$ (Figure $3.2 \mathrm{~A}, \mathrm{R} 2$ ). In addition, the two subsets could be further characterised by their F4/80 expression, with monocytes staining F4/80 int (Figure 3.2A, R3), and neutrophils staining F4/80 (Figure 3.2A, R4). 
Resident macrophages were also harvested from naïve C57Bl/6J mice by peritoneal lavage and stained with the same cell surface markers in order to distinguish them from MSUrecruited monocytes. Resident macrophages expressed high levels of F4/80 (Figure 3.2B, gate R5) and stained negative for both Gr-1 and 7/4 (Figure 3.2B, gate R6).

A
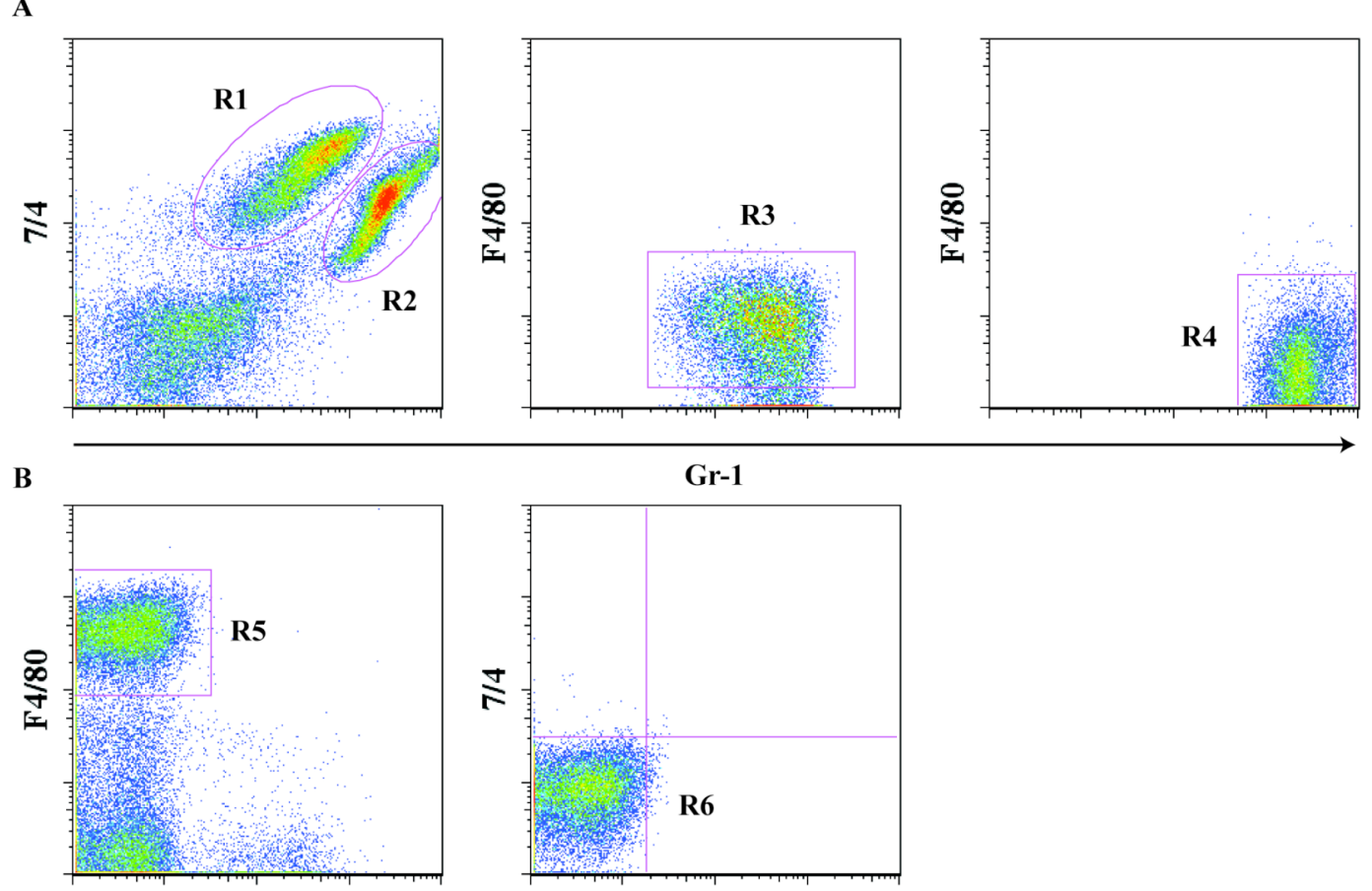

Gr-1

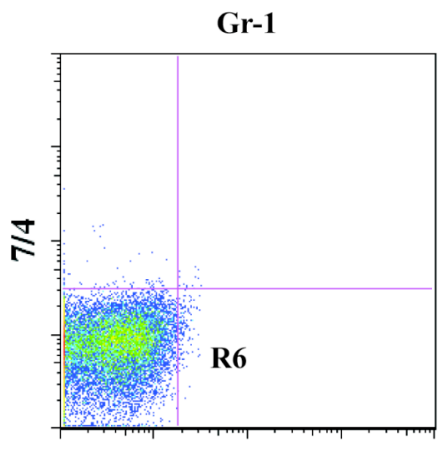

Gr-1

\section{Figure 3.2: Identification of cell types involved in MSU-induced inflammation in vivo.}

$\mathrm{C} 57 \mathrm{Bl} / 6 \mathrm{~J}$ mice were administered with an i.p. injection of MSU crystals $(3 \mathrm{mg} / 0.5 \mathrm{~mL})$. After four hours, peritoneal exudate cells were harvested, stained for cell surface antigen Gr-1, 7/4 and F4/80 and samples of individual mice analysed by flow cytometry. A. Monocytes were identified as Gr- $1^{\text {int }}, 7 / 4^{\text {hi }}$ (gate R1) and F4/80 int (gate R3) while neutrophils were Gr-1 ${ }^{\text {hi }}, 7 / 4^{\text {hi }}$ (gate R2) and F4/80- (gate R4). B. F4/80 hi resident macrophages (gate R5) were 7/4 and Gr-1 ${ }^{-}$(gate R6) was identified in naïve mice. Results are representative of at least three independent experiments.

Therefore, from the analysis of cell surface marker expression, the staining strategies applied were: monocytes $\left(\mathrm{Gr}-1^{\text {int }}, 7 / 4^{\mathrm{hi}}, \mathrm{F} 4 / 80^{\text {int }}\right)$, neutrophils $\left(\mathrm{Gr}-1^{\mathrm{hi}}, 7 / 4^{\mathrm{hi}}, \mathrm{F} 4 / 80^{-}\right)$and macrophages (Gr-1', 7/4- F4/80 ${ }^{\text {hi }}$ ). 


\subsubsection{Depletion of peritoneal resident macrophages with clodronate liposomes}

To investigate the influence of the inflammatory environment on MSU-recruited monocytes, we sought to deplete the resident peritoneal macrophage population using clodronate liposomes $(129,141)$. This process removed the predominant initiators of acute inflammation prior to MSU crystal administration, thereby altering the inflammatory environment into which monocytes were recruited. Encapsulated clodronate (Diagram 3.1A) has been previously used in experimental models to deplete phagocytes both in the blood $(141,144)$ and in local tissues (145). Upon phagocytosis, intracellular lysosomal phospholipases disrupt the phospholipid bilayers of the vesicles, release the drug (Diagram 3.1B) and elicit apoptosis (146-149). Non-specific depletion of cells with limited phagocytic abilities (such as neutrophils and epithelial cells) does not occur as they do not have the necessary intracellular biochemical machinery required to sequester toxic levels of intracellular clodronate (150).

A

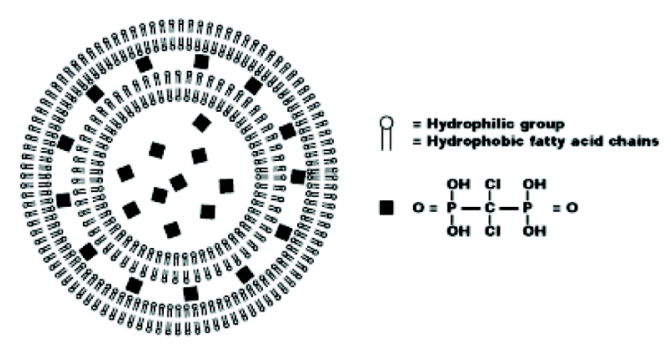

B

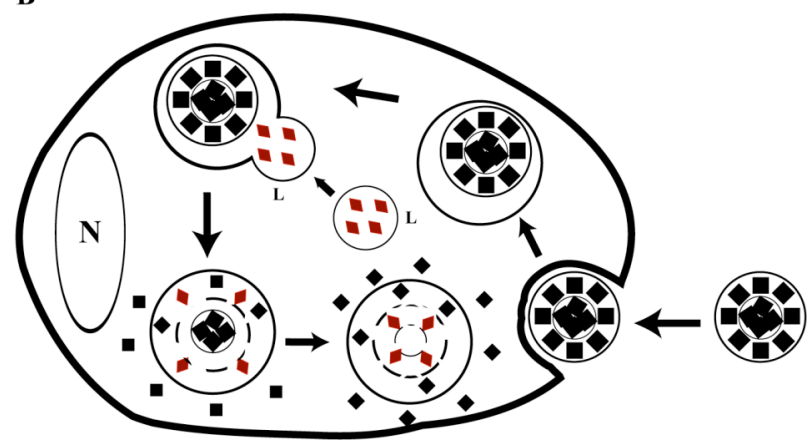

Diagram 3.1: Schematic representation of clodronate liposome uptake by phagocytic

cells. A. Liposomes are artificially prepared fatty vesicles, constructed from phospholipid bilayers separated by aqueous compartments. Hydrophilic molecules such as clodronate dispersed in water are encapsulated during the formation of the liposomes. B. Clodronate encapsulated liposomes (squares) are phagocytosed by macrophages via endocytosis. Fusion with lysosomes (L) results in the disruption of the liposome bilayers by phospholipases (diamonds). Increased amounts of clodronate are released as more concentric bilayers are disrupted. ( $\mathrm{N}=$ macrophage nucleus). Diagram 3.1 was modified from (129).

Preliminary experiments were conducted to determine the time-course and efficiency of the depletion process. Naïve C57B1/6J mice were injected intraperitoneally with a $200 \mu \mathrm{L}$ $(0.7 \mathrm{M})$ solution of clodronate-encapsulated liposomes. As shown in Figure 3.3, liposome 
treatment depleted over $95 \%$ of resident $\mathrm{F} 4 / 80^{+}$cells three days after liposome administration as previously described (141). The small proportion of remaining F4/80 cells observed represented a small number (5\%) of migrating monocytes (Figure 3.3B).

A

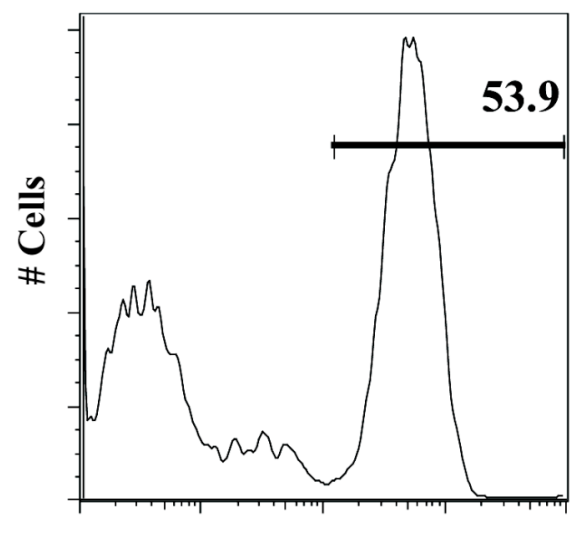

B

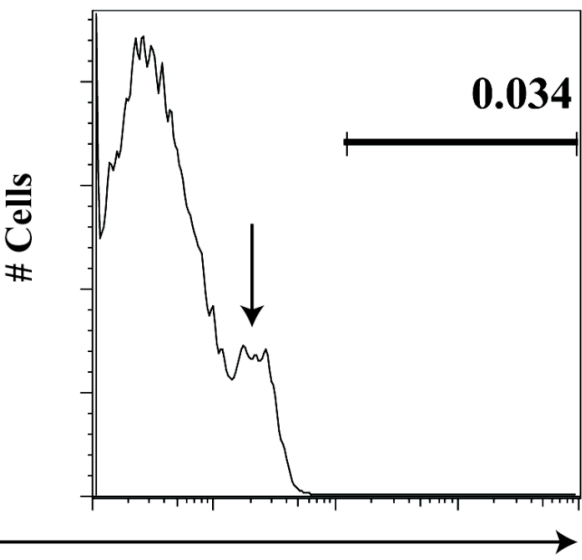

F4/80

Figure 3.3: Clodronate liposome depletion of peritoneal resident macrophages.

C57B1/6J mice were either A. left untreated or B. injected intraperitoneally with $200 \mu \mathrm{L}$ of clodronate liposomes. After three days, peritoneal exudate cells were harvested by peritoneal lavage, stained for cell surface antigens Gr-1 and F4/80 and samples of individual mice analysed by flow cytometry. Arrow represents a small population (5\%) of migrating $\mathrm{F} 4 / 80^{\text {int }}$ cells. Plots are gated on $\mathrm{Gr}^{-}$and $\mathrm{F} 4 / 80^{\mathrm{hi}}$ macrophages. Results are representative of at least three independent experiments.

Therefore, to induce acute inflammation after depleting the resident macrophages, MSU crystal administration was given at three days post intraperitoneal treatment; this represented the time of maximum macrophage depletion.

\subsubsection{Effect of macrophage depletion on MSU-induced leukocyte recruitment}

As the previous depletion experiment was conducted for a single time-point only, we subsequently sought to investigate the effects of macrophage depletion on cell recruitment over the course of an entire MSU-induced inflammatory response.

C57B1/6J mice were either left untreated or injected intraperitoneally with clodronate liposomes. Three days post-liposome treatment, mice were injected i.p. with $3 \mathrm{mg} / 0.5 \mathrm{~mL}$ 
of MSU crystals. At different time-points, PECs were recovered and the total number of viable cells determined using trypan blue exclusion. In the conventional MSU-induced inflammatory model, total cell infiltration peaked at 18 hours after MSU administration and began to resolve after 24 hours (Figure 3.4). However, after the depletion of resident macrophages, the peak of MSU-induced cellular recruitment was significantly abrogated (Figure 3.4).

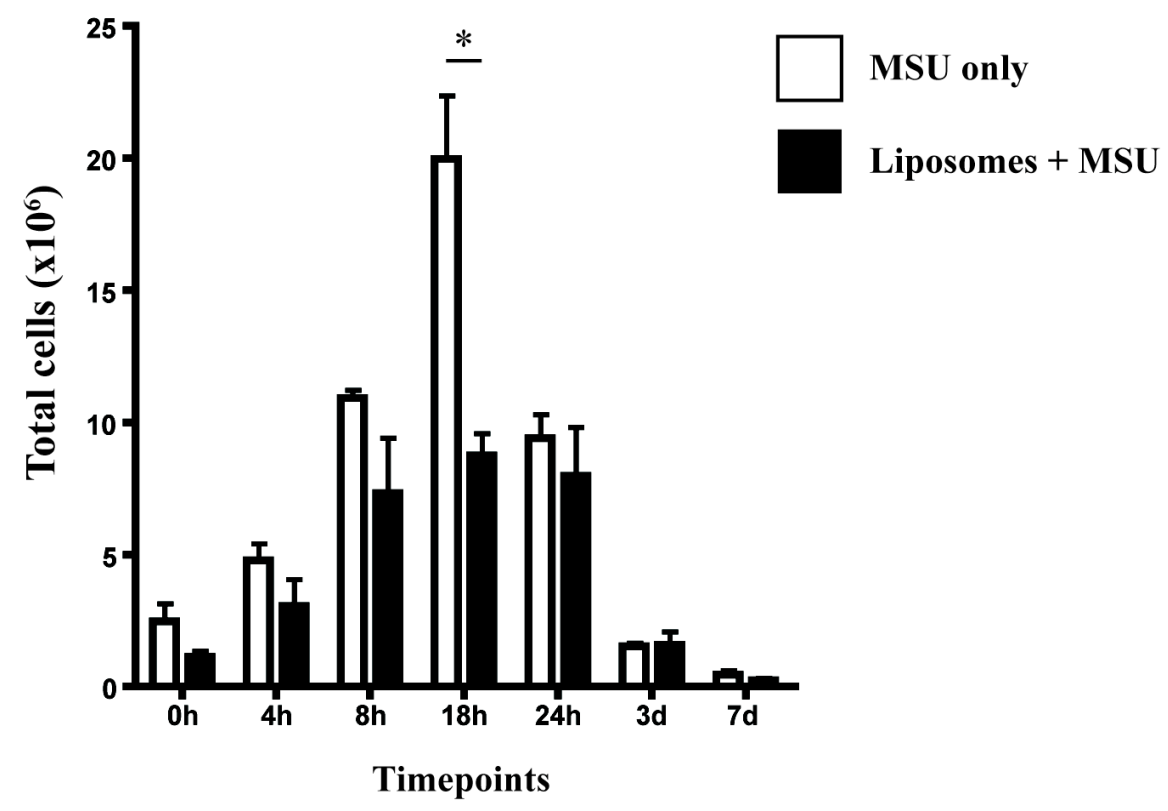

Figure 3.4: Total cell infiltration after clodronate liposome depletion of resident macrophages. $\mathrm{C} 57 \mathrm{Bl} / 6 \mathrm{~J}$ mice were either left untreated or administered with an i.p. injection of $200 \mu \mathrm{L}$ clodronate liposomes. After three days, mice were injected i.p. with MSU crystals $(3 \mathrm{mg} / 0.5 \mathrm{~mL})$. At different time-points, peritoneal exudate cells were harvested and total cell numbers determined by mixing $10 \mu \mathrm{L}$ of cell suspension with $90 \mu \mathrm{L}$ of Trypan Blue Stain. The mixture was placed on a hemocytometer and viable cells that excluded the dye were counted under a microscope. Values represent mean \pm S.E.M. ${ }^{*}=P$ $<0.05$ as determined by two-tailed unpaired Student t-test. Results are representative of two independent experiments with four mice per group.

We next asked the question: whether the change in total cell numbers was due to a general reduction in cell infiltration or a decrease in the recruitment of a specific cell type?

In MSU-induced inflammation, cellular infiltration is largely comprised of monocytes and neutrophils; with the latter been shown to migrate in greater numbers compared to 
monocytes (58). To determine the profile of different infiltrating cells after MSU stimulation, PECs slides were prepared, fixed with DiffQuik staining kit and differential counts performed based on cell morphology as previously described (section 3.2.1). Analysis of recruited leukocyte populations showed that depletion of resident macrophages significantly abrogated neutrophil infiltration into the peritoneum (Figure 3.5).

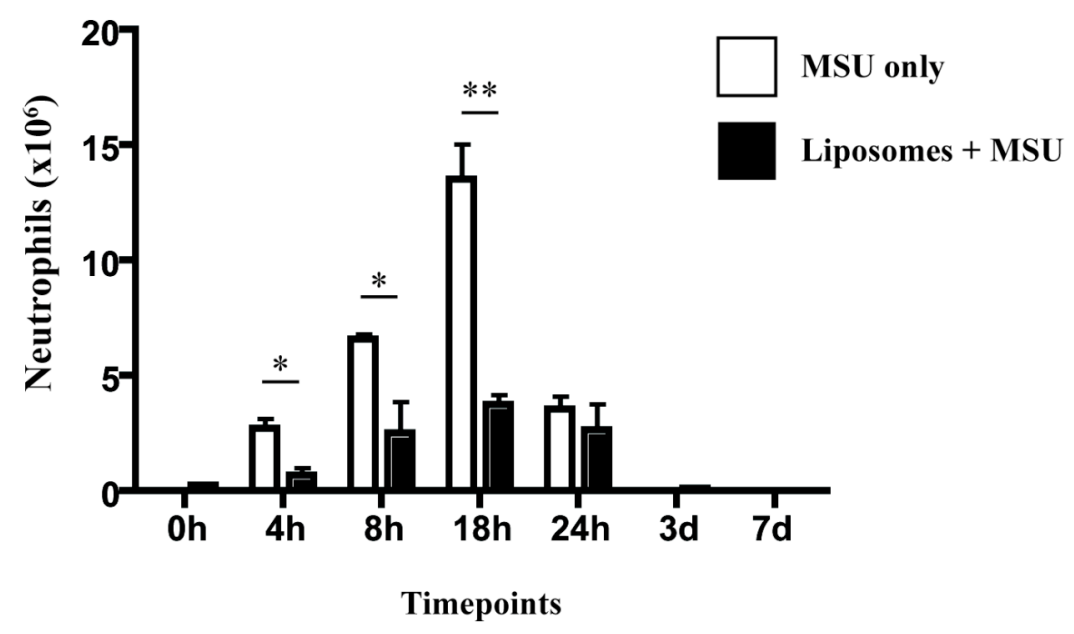

Figure 3.5: Neutrophil recruitment after resident macrophage depletion in MSUinduced inflammation. C57B1/6J mice were either left untreated or administered with an i.p. injection of $200 \mu \mathrm{L}$ clodronate liposomes. After three days, mice were injected i.p. with MSU crystals $(3 \mathrm{mg} / 0.5 \mathrm{~mL})$. At different time-points, peritoneal exudate cells were harvested and $100 \mu 1$ of cell suspension centrifuged onto glass slides using a cytocentrifuge. Cells were then fixed with a DiffQuik staining kit and differential cell counts performed, 200 cells were counted per sample. Values represent mean \pm S.E.M. $*=P<0.05 * *$ $=P<0.005$ as determined by two-tailed unpaired Student t-test. Results are representative of two independent experiments with four mice per group.

Whilst infiltrating neutrophil numbers were significantly reduced after macrophage depletion, the total number of monocytes recruited remained unaffected in vivo (Figure 3.6). The profile of monocyte recruitment was similar between normal and macrophagedepleted mice across the entire inflammatory response (Figure 3.6). 


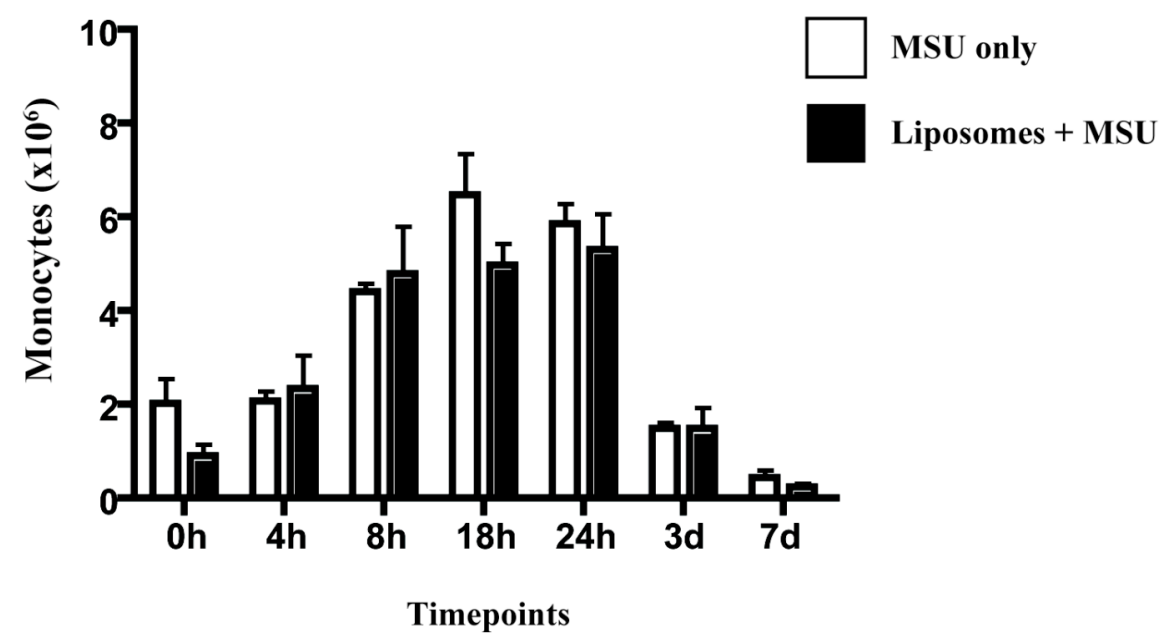

Figure 3.6: Monocyte recruitment after resident macrophage depletion in MSUinduced inflammation. $\mathrm{C} 57 \mathrm{~B} 1 / 6 \mathrm{~J}$ mice were either left untreated or administered with an i.p. injection of $200 \mu \mathrm{L}$ clodronate liposomes. After three days, mice were injected i.p. with MSU crystals $(3 \mathrm{mg} / 0.5 \mathrm{~mL})$. At different time-points, peritoneal exudate cells were harvested and $100 \mu l$ of cell suspension centrifuged onto glass slides using a cytocentrifuge. Cells were then fixed with a DiffQuik staining kit and differential cell counts performed, 200 cells were counted per sample. Values represent mean \pm S.E.M. Results are representative of two independent experiments with four mice per group.

These results were also consistent with our group's previous findings that macrophage activation is responsible for neutrophil but not monocyte recruitment (58).

\subsubsection{Effect of macrophage depletion on the production of MSU- induced cytokines and chemokines}

We next investigated the impact of resident macrophage depletion on the cytokine/chemokine milieu within the peritoneum after MSU stimulation. Analysis of the levels of pro-inflammatory cytokines in the supernatants within the PBS lavage fluid in normal MSU-treated mice showed that IL-1 $\beta$ and IL-6 levels in the peritoneum all peaked within four hours of MSU administration, returning to normal within 24 hours (Figure 3.7A and Figure 3.7B). TNF $\alpha$ showed a biphasic pattern over the course of the inflammatory response (Figure 3.7C). 
Depletion of resident macrophages prior to MSU administration significantly decreased IL-6 and IL-1 $\beta$ production compared to the normal (Figure 3.7A and Figure 3.7B). There were no significant differences in TNF $\alpha$ levels detected between the macrophage-depleted and non-depleted groups (Figure 3.7C).
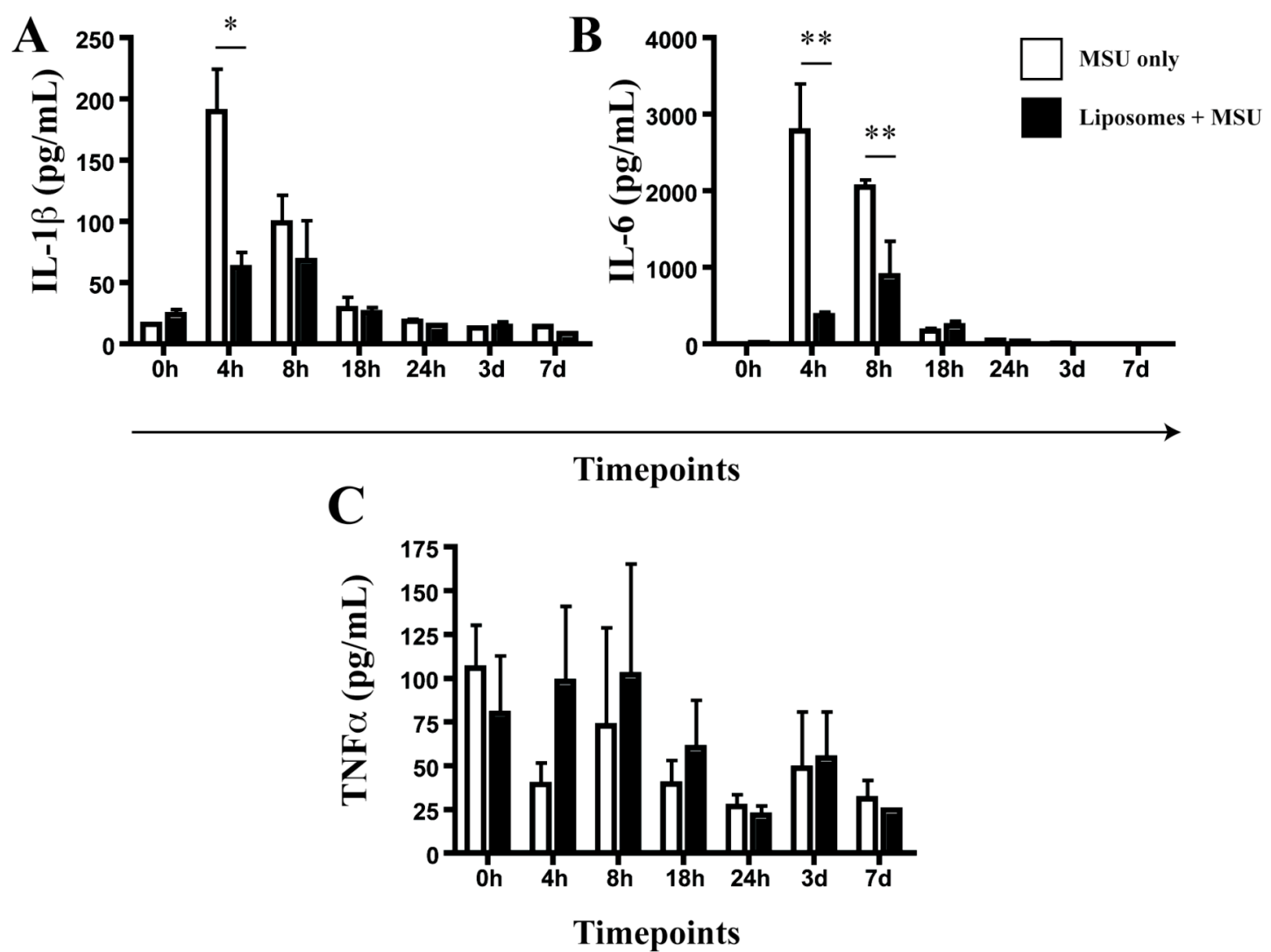

Figure 3.7: Pro-inflammatory cytokines in the peritoneum after MSU administration.

C57B1/6J mice were either left untreated or administered with an i.p. injection of $200 \mu \mathrm{L}$ clodronate liposomes. After three days, mice were injected i.p. with MSU crystals $(3 \mathrm{mg} / 0.5 \mathrm{~mL})$. At different timepoints, peritoneal lavages were performed and samples centrifuged. Supernatants from each sample were collected and levels of IL-1 $\beta$, IL-6 and TNF $\alpha$ determined by ELISA or Bioplex bead array. Values represent mean \pm S.E.M. $*=P<0.05 * *=P<0.005$ as determined by two-tailed unpaired Student $\mathrm{t}$ test. Results are representative of two independent experiments with four mice per group.

As the depletion of resident macrophages decreased neutrophil infiltration in response to MSU stimulation but left monocyte recruitment unaffected, we next looked at the chemokine levels detected in the peritoneal lavage fluid. 
Recruitment of neutrophils into the peritoneum during MSU-induced inflammation is associated with the production of the neutrophil chemokine $\mathrm{KC}$ (the mouse ortholog to the gene encoding human IL-8 (65)), whereas monocyte infiltration is mediated by the chemoattractant MCP-1. Like the pro-inflammatory cytokines, MCP-1 and KC levels in the peritoneum of normal MSU-treated mice also peaked within four hours of MSU administration, and returned to normal levels within 24 hours (Figure 3.8A and Figure $3.8 \mathrm{~B})$.

Following depletion of resident macrophages MSU crystal administration significantly abrogated MCP-1 and KC production in vivo (Figure 3.8A and Figure 3.8B).
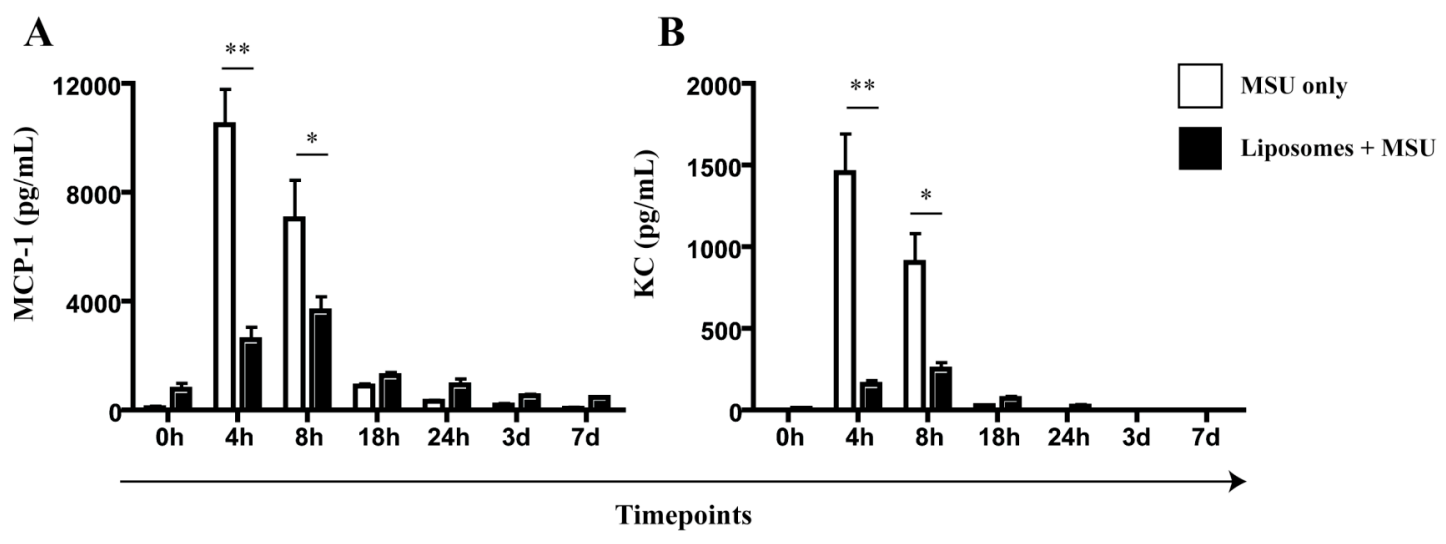

Figure 3.8: Pro-inflammatory chemokines in the peritoneum after MSU

administration. C57B1/6J mice were either left untreated or administered with an i.p. injection of $200 \mu \mathrm{L}$ clodronate liposomes. After three days, mice were injected i.p. with MSU crystals $(3 \mathrm{mg} / 0.5 \mathrm{~mL})$. At different time-points, peritoneal lavages were performed and samples centrifuged. Supernatants from each sample were collected and levels of MCP-1 and KC determined by Bioplex bead array. Values represent mean \pm S.E.M. $*=P<0.05 * *=P<0.005$ as determined by two-tailed unpaired Student $\mathrm{t}$ test. Results are representative of two independent experiments. Results are representative of two independent experiments with four mice per group.

Together these results showed that macrophage depletion abrogates MSU-induced neutrophil infiltration without affecting monocyte recruitment and confirmed that monocytes recruited by MSU-stimulation in the absence of resident macrophages migrate into a significantly less inflammatory environment. This led to the question: do the monocytes migrating into the abrogated inflammatory environment differentiate or function differently to those recruited into a normal inflammatory environment? 


\subsubsection{Cell morphology of MSU-recruited monocytes in normal versus macrophage-depleted mice}

To investigate whether the MSU-recruited monocytes from normal and macrophagedepleted mice exhibited variations in differentiation, normal and clodronate liposometreated $\mathrm{C} 57 \mathrm{Bl} / 6 \mathrm{~J}$ mice were injected with $\mathrm{MSU}$ crystals $(3 \mathrm{mg} / 0.5 \mathrm{~mL})$ and the PECs harvested at different time-points. The different recruited monocyte populations were then characterised based on morphology, cell surface marker expression and functional phenotype.

After four hours, infiltrating monocytes from both normal and macrophage-depleted mice displayed similar cell morphologies (Figure 3.9). However, at 24 hours, monocytes from untreated mice displayed larger cytoplasmic volume compared to monocytes from macrophage-depleted mice (Figure 3.9). One week after MSU administration, monocytes recruited in macrophage-depleted mice were clearly smaller in size than monocytes recruited into a normal MSU-induced inflammatory environment (Figure 3.9). 


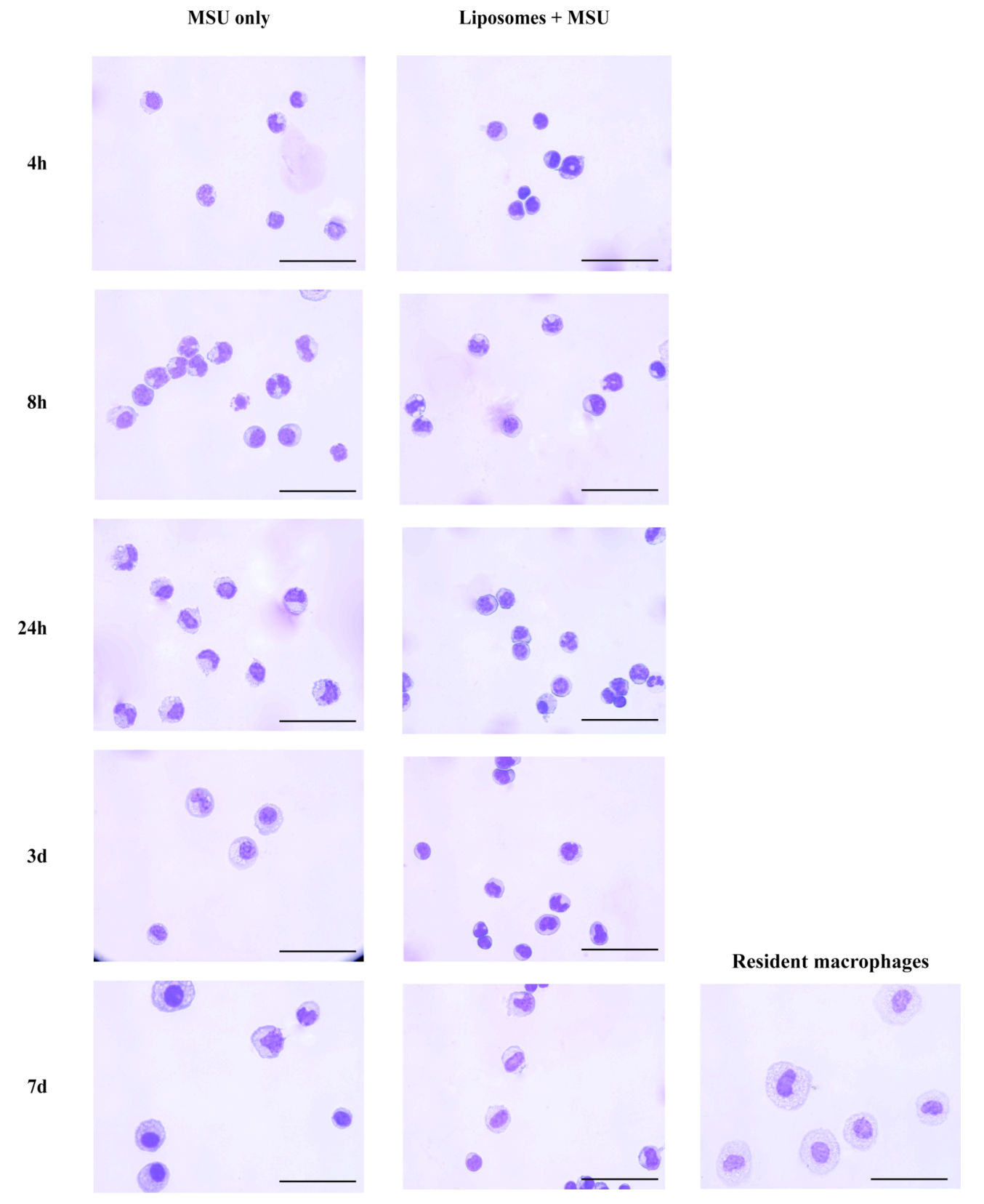

Figure 3.9: Morphology of recruited monocytes during the course of an MSU-induced inflammatory response. C57B1/6J mice were either left untreated or administered with an i.p. injection of $200 \mu \mathrm{L}$ clodronate liposomes. After three days, mice were injected i.p. with MSU crystals $(3 \mathrm{mg} / 0.5 \mathrm{~mL})$. At different time-points, peritoneal exudate cells were harvested and $100 \mu 1$ of cell suspension centrifuged onto glass slides using a cytocentrifuge. Cells were then fixed with a DiffQuik staining kit and analysed under a microscope. Original magnification 100x. Scale bar represents 50um. Results are representative of three independent experiments.

The cell morphologies shown above indicated a delay in MSU-recruited monocyte to macrophage differentiation in macrophage-depleted compared to normal mice. 


\subsubsection{Effects of monocyte transmigration on surface marker expression}

We next sought to investigate whether cell surface markers associated with differentiation correlated with the observed variations in cell morphology.

Before investigating the expression of MSU-recruited monocyte differentiation markers at different time-points along the inflammatory response, the expression of key monocyte/macrophage cell surface antigens on both blood and (4-hour) MSU-recruited monocytes was first studied.

F4/80 is a key macrophage cell surface marker used to distinguish between the different stages of monocyte differentiation. This provides an additional marker in conjunction with other cell surface antigens such as 7/4 and Gr-1 (hemopoietic markers), CD206 (mannose receptor) and CD14 (TLR4 complement molecule) to profile the stages of monocyte to macrophage differentiation after MSU-induced recruitment.

Naïve C57Bl/6J mice were tail-bled and the RBCs lysed with ACT lysis buffer. Cell suspensions were then stained with fluorescent antibodies for the monocyte surface antigens $\mathrm{Gr}-1,7 / 4$, and $\mathrm{CD} 11 \mathrm{~b}$, and also for macrophage differentiation markers $\mathrm{F} 4 / 80$, CD14 and CD206, and analysed by flow cytometry. The same staining protocol was utilized for PECs harvested from mice administered with MSU crystals i.p. for four hours.

MSU-recruited monocytes showed no decrease in the hemopoietic cell marker Gr-1; however, 7/4 was significantly downregulated (Figure 3.10). In terms of the differentiation markers, F4/80 expression on monocytes was unaltered following recruitment whilst CD14 expression increased and CD11b expression decreased (Figure 3.10). CD206 expression was barely detectable on both blood and recruited monocytes (Figure 3.10). These data indicated that the process of recruitment alone alters inflammatory monocyte surface phenotype. 
Gr-1

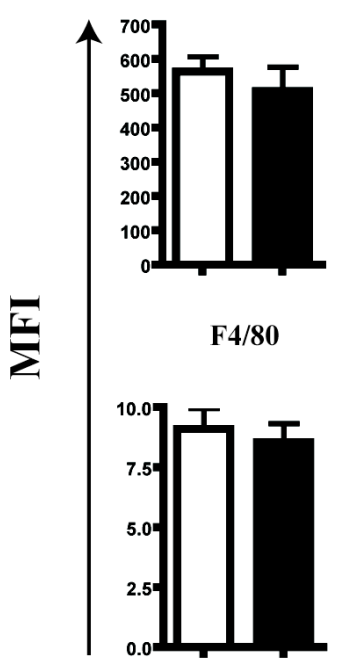

$7 / 4$

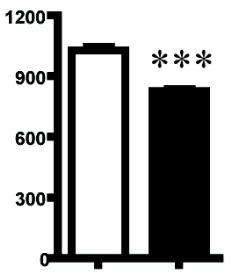

CD14

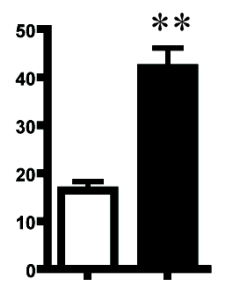

CD11b

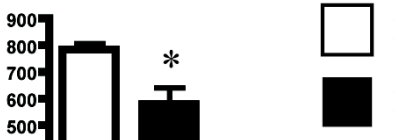

Blood monocytes

Recruited monocytes

Figure 3.10: The process of migrating from the bloodstream through the endothelium alters inflammatory monocyte surface marker expression. C57B1/6J mice were tail-bled prior to being administered with an i.p. injection of MSU crystals $(3 \mathrm{mg} / 0.5 \mathrm{~mL})$. After four hours, peritoneal exudate cells were harvested, stained for cell surface antigens and samples of individual mice analysed by flow cytometry. Plots are gated on $\mathrm{Gr} 1^{\text {in }}$ and $\mathrm{F} 4 / 80^{\text {int }}$ monocytes. Values represent mean \pm S.E.M. $*=P<$ $0.05^{* *}=P<0.005 * * *=P<0.0005$ as determined by two tailed unpaired Student t-test. Results are representative of at least three independent experiments with three mice per group.

\subsubsection{Changes in surface marker expression of monocytes recruited into a macrophage-depleted environment}

In order to further compare the profile of MSU-recruited monocytes from normal and macrophage-depleted mice, PECs were isolated from normal and macrophage-depleted mice at different time-points and the surface phenotypes compared. In the normal MSUinduced inflammation model, recruited $\mathrm{F} 4 / 80^{\mathrm{int}}$ monocytes rapidly downregulated hemopoietic cell surface markers Gr-1 and 7/4 within $24-72$ hours after recruitment (Figure 3.11). Monocytes recruited into a macrophage-depleted environment showed a trend towards a delay in the downregulation of these surface antigens (Figure 3.11).

As observed previously in section 3.2 .2 , the small proportion of $F 4 / 80^{\text {int }}$ monocytes $(5 \%)$ present in the peritoneum of macrophage-depleted mice at 0-hours was also observed during flow cytometry analysis. 
Gr-1

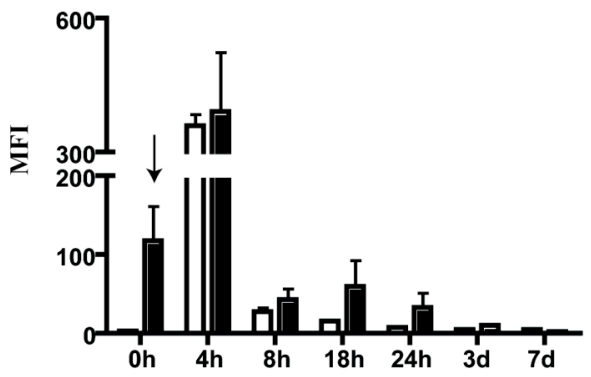

$7 / 4$

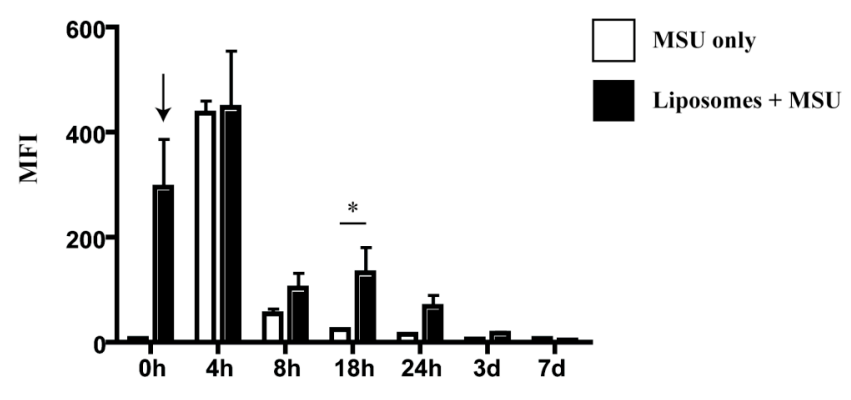

Timepoints

Figure 3.11: Hemopoietic cell surface markers of monocytes recruited into a macrophage-depleted environment. $\mathrm{C} 57 \mathrm{Bl} / 6 \mathrm{~J}$ mice were either left untreated or administered with an i.p. injection of $200 \mu \mathrm{L}$ clodronate liposomes. After three days, mice were injected i.p. with MSU crystals $(3 \mathrm{mg} / 0.5 \mathrm{~mL})$. At different time-points, peritoneal exudate cells were harvested,stained for cell surface antigens Gr-1 and 7/4 and samples of individual mice analysed by flow cytometry. Arrows represent a small population (5\%) of migrating $\mathrm{F} 4 / 80^{\text {int }}$ cells. Plots are gated on $\mathrm{Gr} 1^{\text {in }}$ and $\mathrm{F} 4 / 80^{\text {int }}$ monocytes. Values represent mean \pm S.E.M. $*=P<0.05$ as determined by two tailed unpaired Student t-test. Results are representative of two independent experiments with four mice per group.

Following MSU-administration, infiltrating monocytes were analysed for their expression of common macrophage differentiation markers (F4/80, CD14, CD11b and CD206). Resident macrophages were used as a positive control $\left(\mathrm{F} 4 / 80^{\mathrm{hi}}, \mathrm{CD} 14^{\mathrm{hi}}, \mathrm{CD} 11 \mathrm{~b}{ }^{\mathrm{hi}}\right.$ and $\mathrm{CD}^{206^{\mathrm{hi}}}$ ) (Figure 3.12, 0h). MSU-recruited monocytes from both normal and macrophage-depleted mice increased surface expression of F4/80, and CD11b as well as the mannose receptor CD206 and the TLR4 complement molecule CD14 over-time with no significant differences between the two profiles (Figure 3.12). 
F4/80

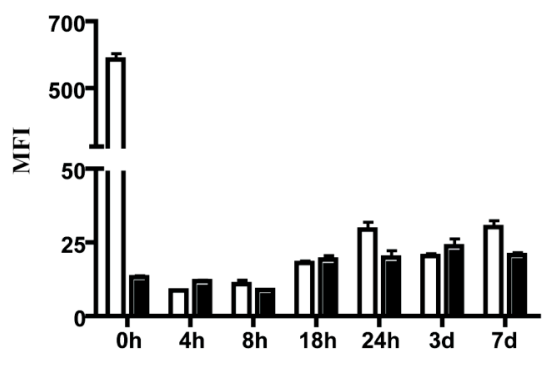

CD14

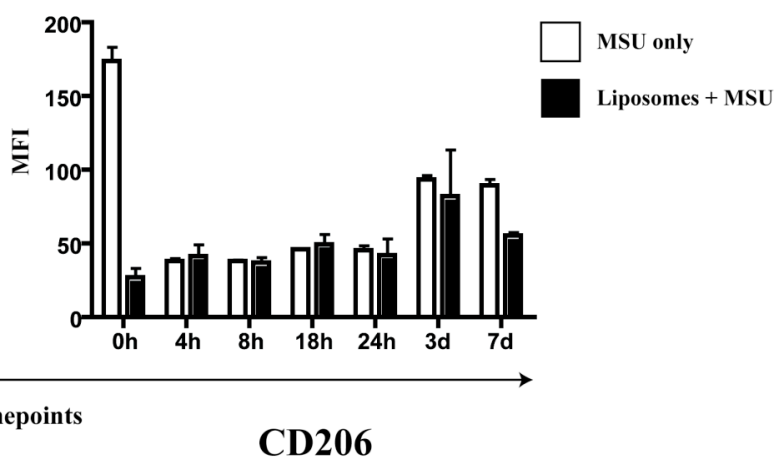

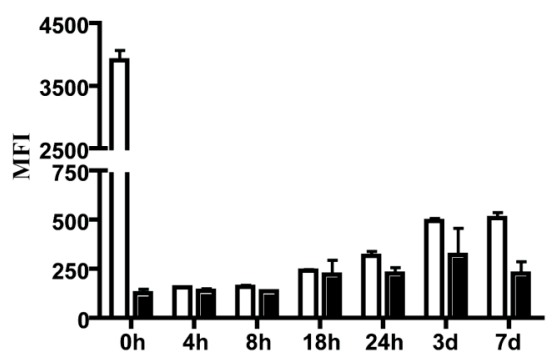

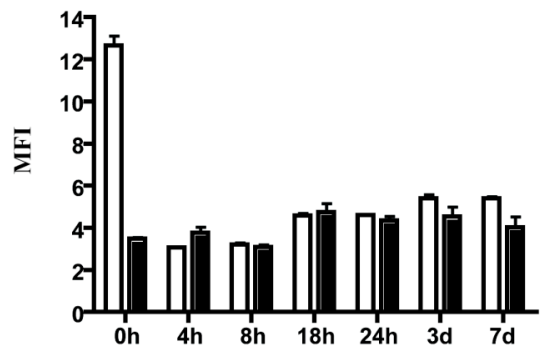

Timepoints

\section{Figure 3.12: Differentiation cell surface markers of monocytes recruited into a} macrophage-depleted environment. $\mathrm{C} 57 \mathrm{Bl} / 6 \mathrm{~J}$ mice were either left untreated or administered with an i.p. injection of $200 \mu \mathrm{L}$ clodronate liposomes. After three days, mice were injected i.p. with MSU crystals $(3 \mathrm{mg} / 0.5 \mathrm{~mL})$. At different time-points, peritoneal exudate cells were harvested, stained for cell surface antigens F4/80, CD14, CD11b and CD206 and samples of individual mice analysed by flow cytometry. Plots are gated on $\mathrm{Gr} 1^{\text {in }}$ and $\mathrm{F} 4 / 80^{\text {int }}$ monocytes. Values represent mean \pm S.E.M. Results are representative of two independent experiments with four mice per group.

In summary, MSU-recruited monocytes entering a macrophage-depleted environment showed no significant trends towards delayed surface marker expression associated with monocyte to macrophage differentiation. 


\subsubsection{The effect of macrophage depletion on MSU-recruited monocyte function}

We next sought to investigate whether MSU-induced recruitment of monocytes into a normal compared to a macrophage-depleted environment altered monocyte function.

Normal or macrophage-depleted mice were administered MSU i.p. and at different timepoints PECs were harvested. Recruited monocytes were purified using a Lympholyte ${ }^{\circledR}-\mathrm{M}$ density gradient $\left(>75 \%\right.$ monocytes based on flow cytometry analysis of F4/80 ${ }^{\text {int }} \mathrm{Gr}-1^{\text {hi }}$ population). Purified MSU-recruited monocytes were then cultured ex vivo in the presence of MSU crystals $(500 \mu \mathrm{g} / \mathrm{mL})$ or LPS $(100 \mathrm{ng} / \mathrm{mL})$, and the supernatant analysed for proinflammatory cytokines by ELISA or Bioplex bead array. As a positive control, resident macrophages were harvested from naïve mice and stimulated with MSU crystals or LPS (Figure 3.13A and Figure 3.13B, 0h).

MSU-recruited monocytes from normal mice at the early stages of inflammation (4 hours) did not produce pro-inflammatory cytokines after MSU restimulation. However, as the inflammatory response progressed (8 hours onwards), MSU-recruited monocytes increased their capacity to produce IL-1 $\beta$ and IL-6 (Figure 3.13A and Figure 3.13B). Similar trends were observed for LPS restimulation.

Monocytes recruited into a macrophage-depleted environment did not produce IL-1 $\beta$ and IL-6 after MSU restimulation until time-points 24 hours to 1 week (Figure 3.13A and Figure 3.13B).

While there was no significant difference in LPS elicited IL-1 $\beta$ production by recruited monocytes from macrophage-depleted mice compared to normal mice (Figure 3.13A). Recruited monocytes from macrophage-depleted mice produced significantly less IL-6 following restimulation 18 hours after MSU administration (Figure 3.13A). 


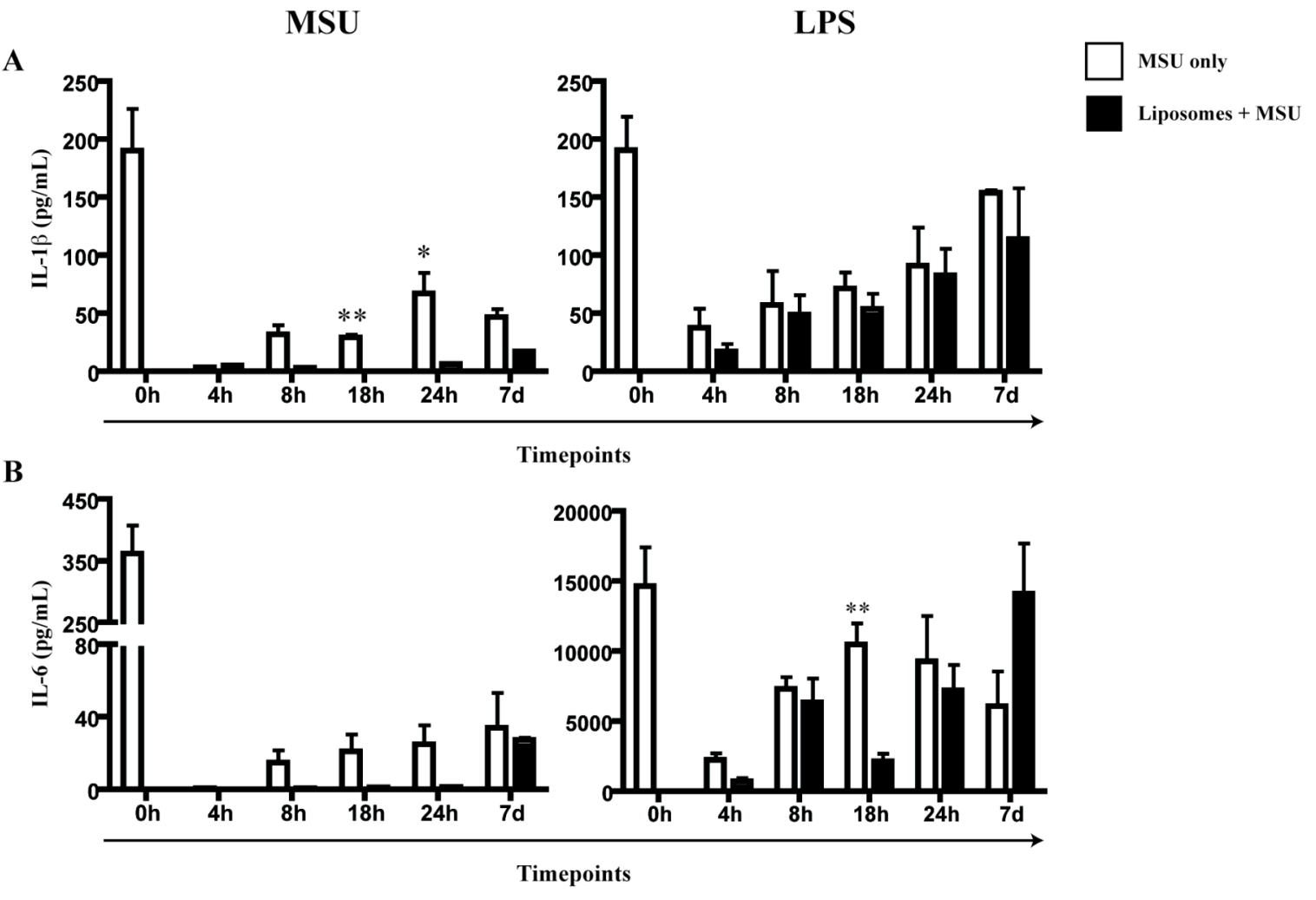

Figure 3.13: Production of pro-inflammatory cytokines by recruited monocytes after

restimulation. C57B $1 / 6 \mathrm{~J}$ mice were either left untreated or administered with an i.p. injection of $200 \mu \mathrm{L}$ clodronate liposomes. After three days, mice were injected i.p. with MSU crystals $(3 \mathrm{mg} / 0.5 \mathrm{~mL})$. At different time-points, peritoneal exudate cells were harvested and purified by Lympholyte ${ }^{\circledR}-\mathrm{M}$ density gradient ( $>75 \%$ monocytes). Cells were resuspended at $5 \times 10^{5}$ cells $/ \mathrm{mL}$ in cRPMI and $200 \mu \mathrm{L}$ was cultured in the presence of MSU $(500 \mathrm{\mu g} / \mathrm{mL})$ or LPS $(100 \mathrm{ng} / \mathrm{mL})$ for eight hours. After the incubation period, levels of IL- $1 \beta$ and IL- 6 were determined by ELISA and Bioplex bead array. Negative controls for IL-1 $\beta(1-5 \mathrm{pg} / \mathrm{mL})$ and IL-6 $(5-15 \mathrm{pg} / \mathrm{mL})$ were omitted from the graph. Measurements performed in quadruplicate. Values represent mean \pm S.E.M. $*=P<0.05 * *=P<0.005$ as determined by one-way ANOVA and Tukey multiple comparison test. Results are representative of the accumulated data from two independent experiments with eight mice per group. 
The capacity of recruited monocytes to produce the chemoattractants MCP-1 and KC after restimulation with MSU crystals or LPS was next analyzed. Again as a positive control, resident macrophages were shown to produce large amounts of both MCP-1 and KC after stimulation with MSU crystals or LPS (Figure 3.14A and Figure 3.14B, 0h).

Monocytes recruited into a normal MSU-induced inflammatory environment did not respond to MSU or LPS restimulation immediately after migration into the peritoneum. However, as the inflammatory response progressed, recruited monocytes produced both MCP-1 and KC in response to MSU crystal and LPS restimulation; this ability to produce MCP-1 was lost after one week.

Monocytes recruited into a macrophage-depleted environment produced lower levels of MCP-1 and $\mathrm{KC}$ in response to MSU restimulation compared to monocytes recruited into a normal MSU-induced inflammatory environment (Figure 3.14A and Figure 3.14B).

Exposure of early (4-hour) recruited-monocytes from macrophage-depleted mice to LPS elicited low levels of MCP-1 compared to normal mice, however this ability was regained at later time-points during the inflammatory response (Figure 3.14A). Production of KC by MSU-recruited monocytes from macrophage-depleted mice restimulated with LPS was significantly abrogated 18 hours after MSU stimulation (Figure 3.14B). 

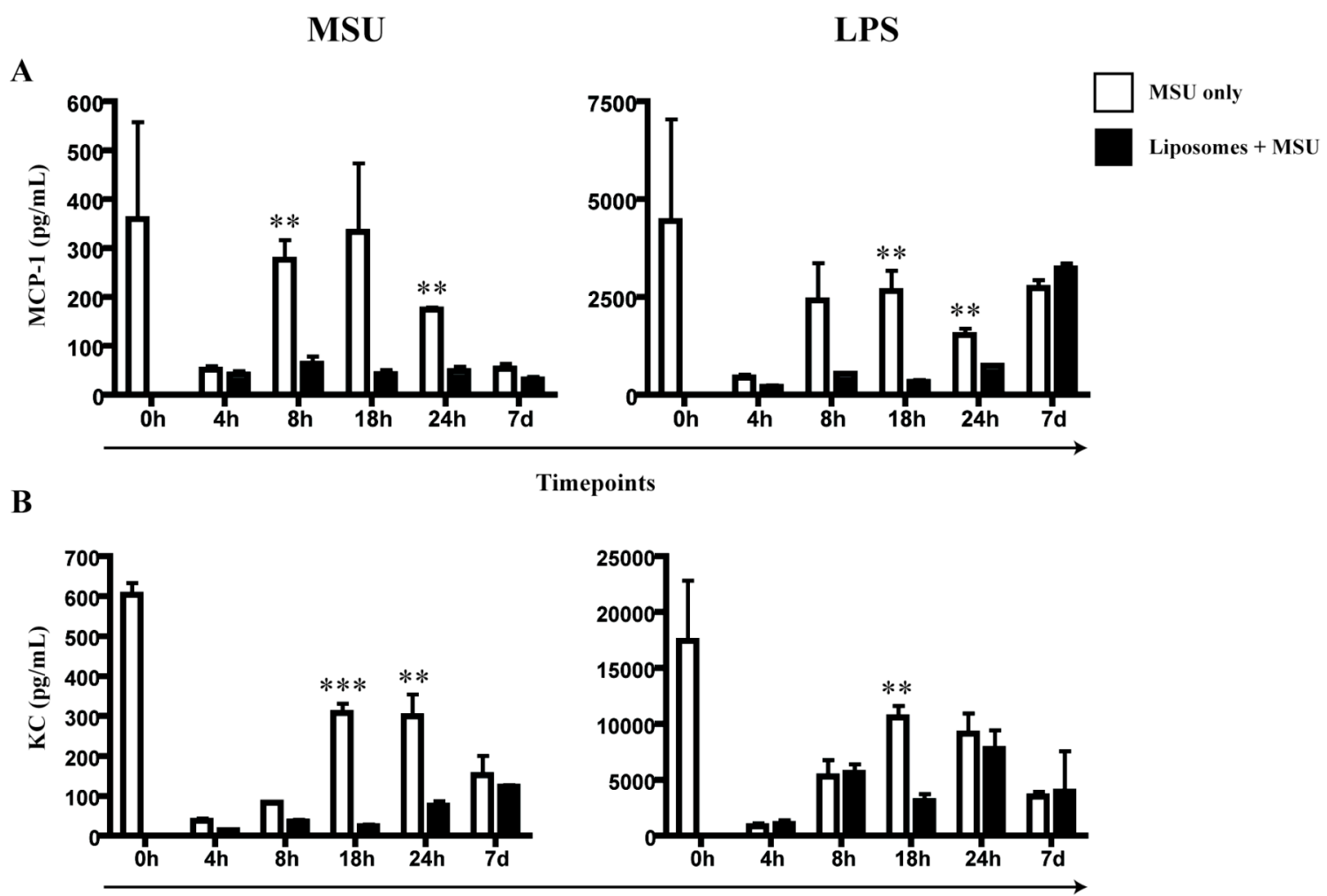

Timepoints

Figure 3.14: Production of pro-inflammatory chemokines by recruited monocytes

after restimulation. $\mathrm{C} 57 \mathrm{Bl} / 6 \mathrm{~J}$ mice were either left untreated or administered with an i.p. injection of $200 \mu \mathrm{L}$ clodronate liposomes. After three days, mice were injected i.p. with MSU crystals ( $3 \mathrm{mg} / 0.5 \mathrm{~mL})$. At different time-points, peritoneal exudate cells were harvested and purified by Lympholyte ${ }^{\circledR}-\mathrm{M}$ density gradient ( $>75 \%$ monocytes). Cells were resuspended at $5 \times 10^{5}$ cells $/ \mathrm{mL}$ in cRPMI and $200 \mu \mathrm{L}$ was cultured in the presence of MSU $(500 \mathrm{\mu g} / \mathrm{mL})$ or LPS $(100 \mathrm{ng} / \mathrm{mL})$ for eight hours. After the incubation period, levels of MCP-1 and KC were determined by Bioplex bead array. Negative controls for MCP-1 (20-80pg/mL) and $\mathrm{KC}(20-70 \mathrm{pg} / \mathrm{mL})$ were omitted from the graph. Measurements performed in quadruplicate. Values represent mean \pm S.E.M. $* *=P<0.005 * * *=P<0.0005$ as determined one-way ANOVA and Tukey multiple comparison test. Results are representative of the accumulated data from two independent experiments with eight mice per group.

In summary, these results demonstrated that purified MSU-recruited monocytes from normal mice had increased ability to produce pro-inflammatory cytokines over time and transiently produced MCP-1 and KC. Recruited monocytes from the macrophage-depleted model displayed an abrogated ability to produce cytokine/chemokines in response to MSU restimulation. In addition, there were fundamental differences in the functional responses of recruited monocytes depending on the stimulus (MSU crystals or LPS). 


\subsubsection{Changes in phagocytic function of monocytes recruited into an abrogated inflammatory environment}

The development of phagocytic ability is an important characteristic of monocyte/macrophage differentiation. Expression of the receptor CD206 is a commonly used determinant of phagocytic ability. However, as CD206 is a receptor used to recognize mannose sugars on bacterial cell walls rather than crystals and was not upregulated on MSU-recruited monocytes, a more relevant method of measuring phagocytosis was deemed necessary. Fluorescent bead uptake was therefore used as an alternative approach to determine phagocytic function.

PECs were recovered at different time-points after MSU administration and monocytes isolated by density gradient separation. Purified MSU-recruited monocytes were incubated with fluorescent beads for 30 minutes then stained for the surface marker F4/80 and analysed by flow cytometry. Phagocytic ability was measured as the percentage of $\mathrm{F} 4 / 80^{+}$ bead $^{+}$cells in culture. In Figure 3.15 and Figure $3.16(0 \mathrm{~h}) \mathrm{F} 4 / 80^{\text {hi }}$ resident macrophages were used as a positive control $\left(43 \%\right.$ bead $\left.^{+}\right)$.

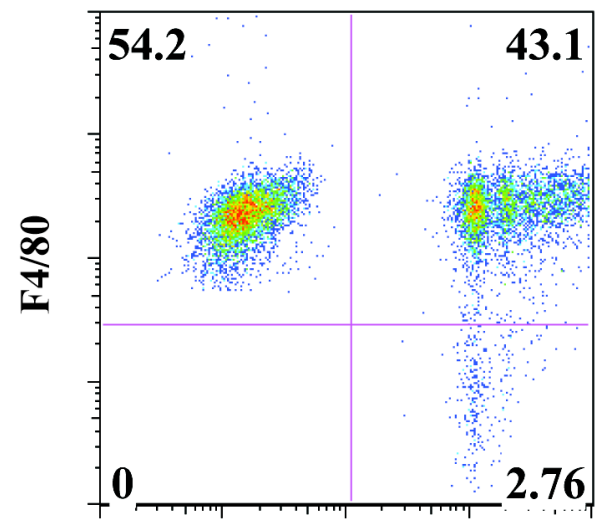

Beads

Figure 3.15: Phagocytosis of fluorescent beads by monocytes/macrophages. Naïve resident macrophages were harvested by peritoneal lavage from C57B1/6J mice. Cells were resuspended at $5 \times 10^{5}$ cells $/ \mathrm{mL}$ and $2 \mathrm{~mL}$ of the suspension was incubated with fluorescent beads for 30 minutes. Excess beads were washed off, cells were stained for the cell surface antigen F4/80 and samples of individual mice analysed by flow cytometry. Results are representative of one experiment with four mice per group. 
As shown in Figure 3.16, approximately $30 \%$ of MSU-recruited F4/80 int monocytes (at 4hours) isolated from normal mice displayed bead uptake, increasing to almost $50 \%$ bead $^{+}$ after 24-hours.

MSU-recruited monocytes purified from macrophage-depleted mice exhibited slightly lower and slower induction of phagocytic ability compared to monocytes from untreated mice, only beginning to show increased uptake of fluorescent beads between $18-24$ hours (Figure 3.16).

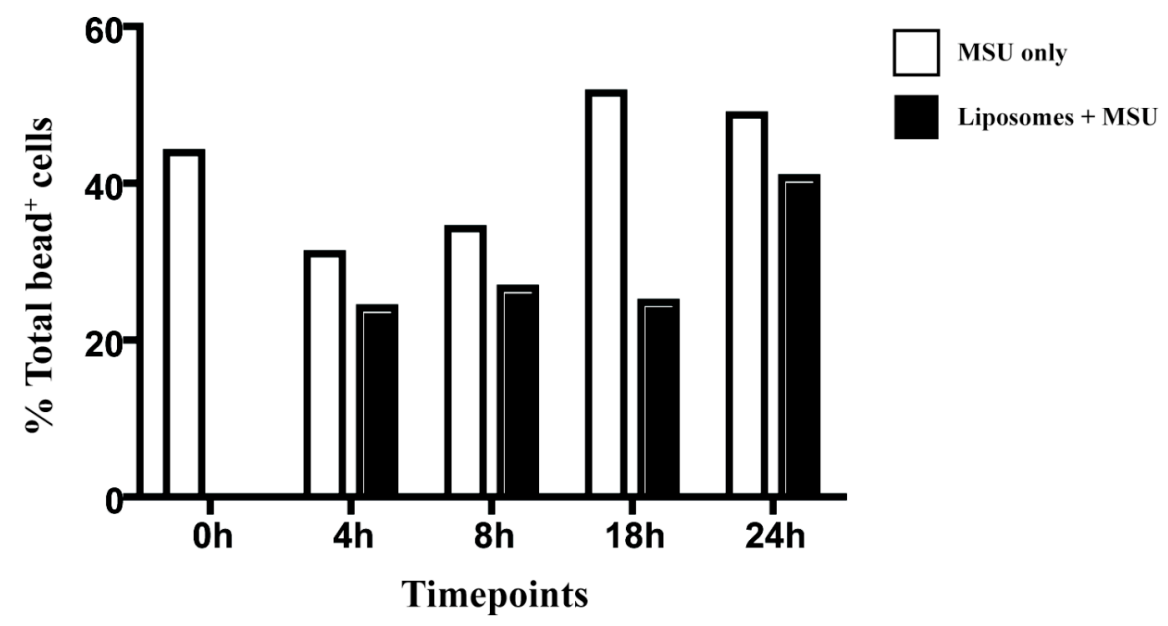

Figure 3.16: MSU-recruited monocytes from macrophage-depleted mice display reduced phagocytic ability. $\mathrm{C} 57 \mathrm{~B} 1 / 6 \mathrm{~J}$ mice were either left untreated or administered with an i.p. injection of $200 \mu \mathrm{L}$ clodronate liposomes. After three days, mice were injected i.p. with MSU crystals $(3 \mathrm{mg} / 0.5 \mathrm{~mL})$. At different time-points, peritoneal exudate cells were harvested and purified by Lympholyte ${ }^{\circledR}$-M density gradient ( $>75 \%$ monocytes). Cells were resuspended at $5 \times 10^{5}$ cells $/ \mathrm{mL}$ and $2 \mathrm{~mL}$ of the suspension was incubated with fluorescent beads for 30 minutes. Excess beads were washed off, cells were stained for the cell surface antigen F4/80 and samples of individual mice analysed by flow cytometry. Values are representative of pooled samples. Plots are gated on $\mathrm{F} 4 / 80^{\text {int }}$ monocytes. Results are representative of one experiment with four mice per group.

Upon observing a delay in phagocytic ability, the capacity of recruited monocyte phagocytosis i.e. the percentage of phagocytic cells to take up more than one fluorescent bead was subsequently exammed. This involved gating on the bead ${ }^{+}$population and then sub-gating on subpopulations displaying a second fluorescent spike. In resident 
macrophages, $52 \%$ of $\mathrm{F} 4 / 80^{+}$bead $^{+}$cells were able to phagocytose multiple beads (Figure 3.17 and Figure 3.18, 0h).

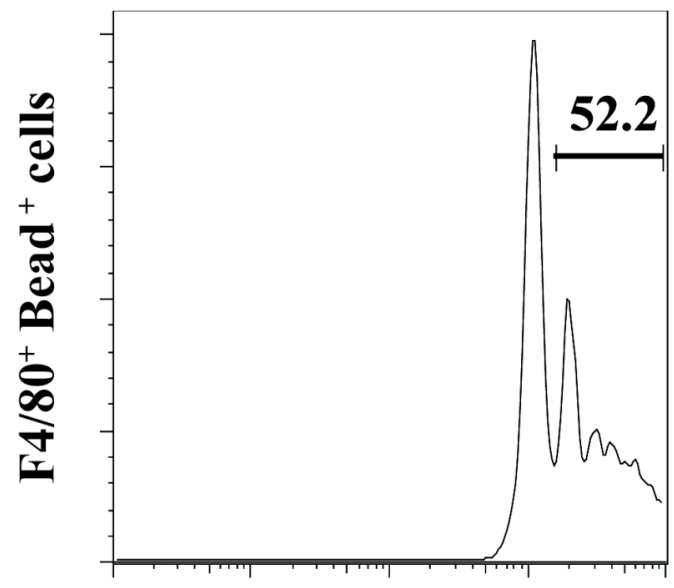

\section{Beads}

Figure 3.17: Phagocytic capacity of monocytes/macrophages. Naïve resident macrophages were harvested by peritoneal lavage from C57B1/6J mice. Cells were resuspended at $5 \times 10^{5}$ cells $/ \mathrm{mL}$ and $2 \mathrm{~mL}$ of the suspension was incubated with fluorescent beads for 30 minutes. Excess beads were washed off, cells were stained for the cell surface antigen F4/80 and samples of individual mice analysed by flow cytometry. Plots are gated on $\mathrm{F} 4 / 80^{+} \mathrm{Bead}^{+}$macrophages. Results are representative of one experiment with four mice per group.

Compared with monocytes isolated from the normal MSU-induced inflammatory response, $\mathrm{F} 4 / 80^{\text {int }}$ bead $^{+}$monocytes from macrophage-depleted mice displayed a delayed development of the ability to take up multiple fluorescent beads at 18-hours, only reaching similar phagocytic capacities to MSU-recruited monocytes from normal mice after 24hours (Figure 3.18). 


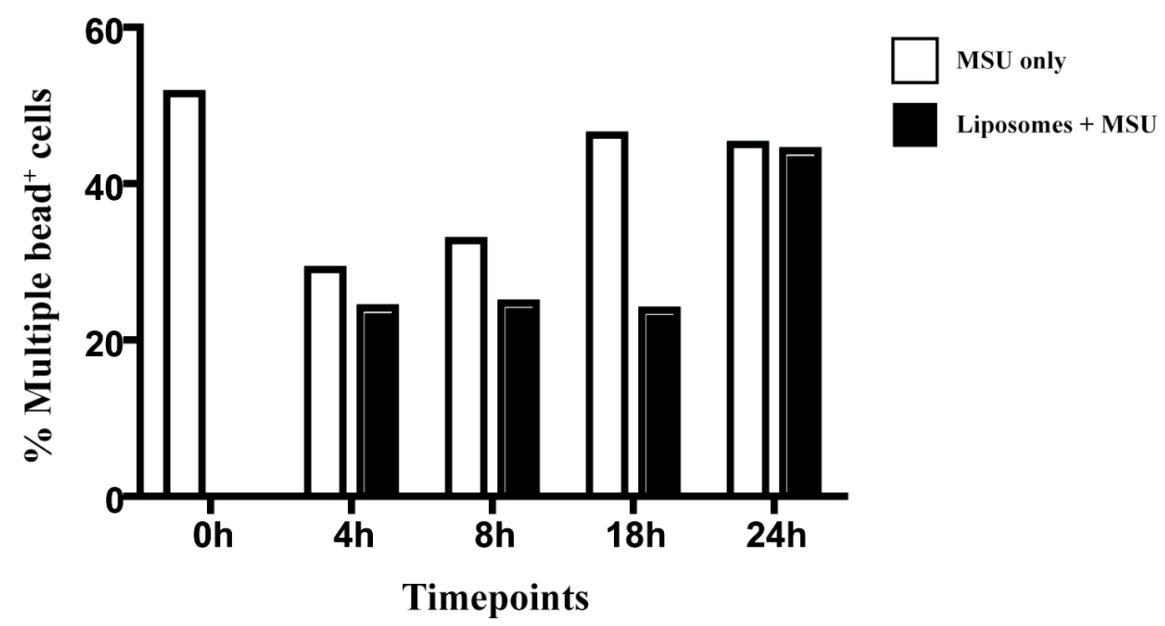

Figure 3.18: Development of the capacity to phagocytose multiple fluorescent beads by monocytes recruited into a macrophage-depleted environment. Total $\mathrm{F} 4 / 80^{+}$and $\mathrm{Bead}^{+}$ cells in Figure 3.16 were analysed for their ability to phagocytose more than one bead using histogram peaks in flow cytometry. Measurements were performed in duplicate. Values are representative of pooled samples. Results are representative of one experiment with four mice per group.

In summary, in normal mice MSU-recruited monocytes increased phagocytic function with time but, this profile was delayed for recruited monocytes from macrophage-depleted mice

\subsubsection{Changes in growth factors detected in the local environment after depletion of resident macrophages}

The next approach was to investigate the possible mechanisms responsible for the observed delay in recruited monocyte differentiation. Colony-stimulating factors (CSFs) such as granulocyte/macrophage colony-stimulating factor (GM-CSF) and macrophage colony-stimulating factor (M-CSF) are critical haematopoietic-cell growth factors. They are defined by their ability to generate in vitro colonies of mature myeloid cells from bonemarrow precursors; both granulocytic and macrophage colonies in the case of GM-CSF and macrophages alone for M-CSF (151). This lead to the question: were CSF levels altered in the macrophage depletion model?

To answer this question, GM-CSF and M-CSF levels were measured in the peritoneal lavage and different time-points after MSU administration in normal and macrophagedepleted mice. In the normal MSU-induced inflammatory model, M-CSF peaked at four 
hours and returned to normal levels within three days (Figure 3.19). Interestingly, the depletion of resident macrophages caused elevated levels of background M-CSF before MSU administration (Figure 3.19, 0h). Four hours after MSU-administration, M-CSF levels dropped in macrophage-depleted mice and increased again at eight hours before slowly returning to baseline levels (Figure 3.19).

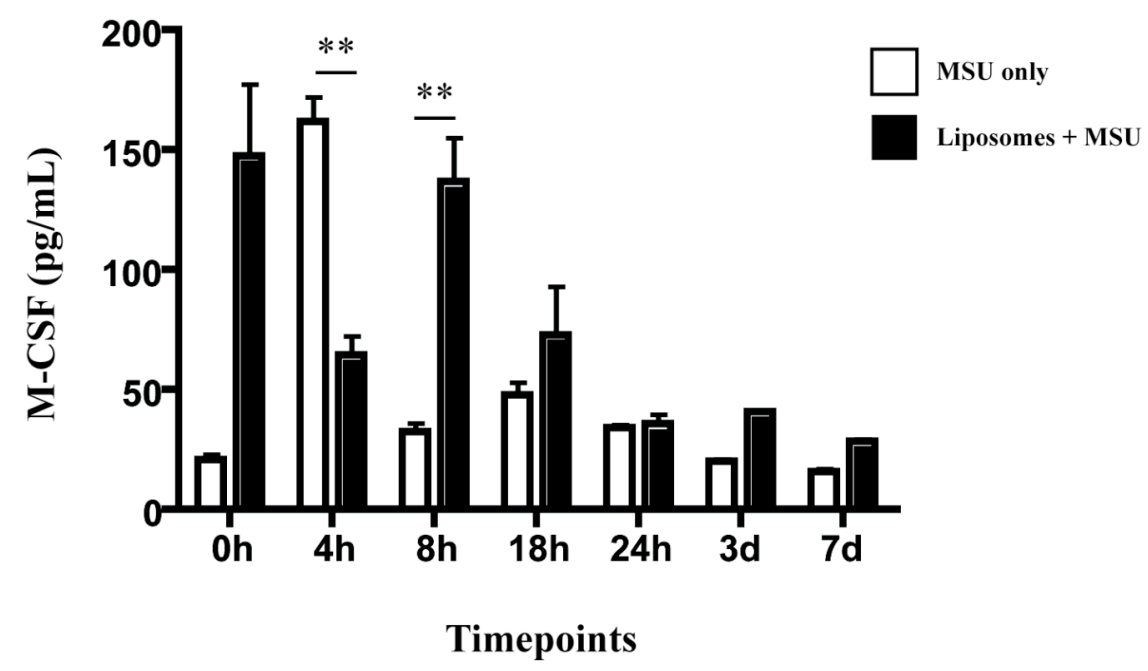

Figure 3.19: Macrophage-Colony Stimulating Factor levels in macrophagedepleted/non-depleted mice during MSU-induced inflammation. C57Bl/6J mice were either left untreated or administered with an i.p. injection of $200 \mu \mathrm{L}$ clodronate liposomes. After three days, mice were injected i.p. with MSU crystals $(3 \mathrm{mg} / 0.5 \mathrm{~mL}$ ). At different time-points, peritoneal lavages were performed and samples centrifuged. Supernatants from each sample were collected and levels of GM-CSF determined by ELISA. Plates were read after 25 minutes. Values represent mean \pm S.E.M. Values represent mean \pm S.E.M. $*=P<0.05 * *=P<0.005$ as determined by two tailed unpaired Student $\mathrm{t}$-test. Results are representative of one experiment with four mice per group. ELISA was conducted by Dr Mischa Walton.

In normal mice, GM-CSF levels decreased over 24 hours following MSU administration and slowly increased again over the course of one week (Figure 3.20). In macrophagedepleted mice, GM-CSF levels were significantly lower following MSU administration (4and 8-hours) compared to normal mice, but showed a similar pattern of recovery during latter stages of the inflammatory response (Figure 3.20). 


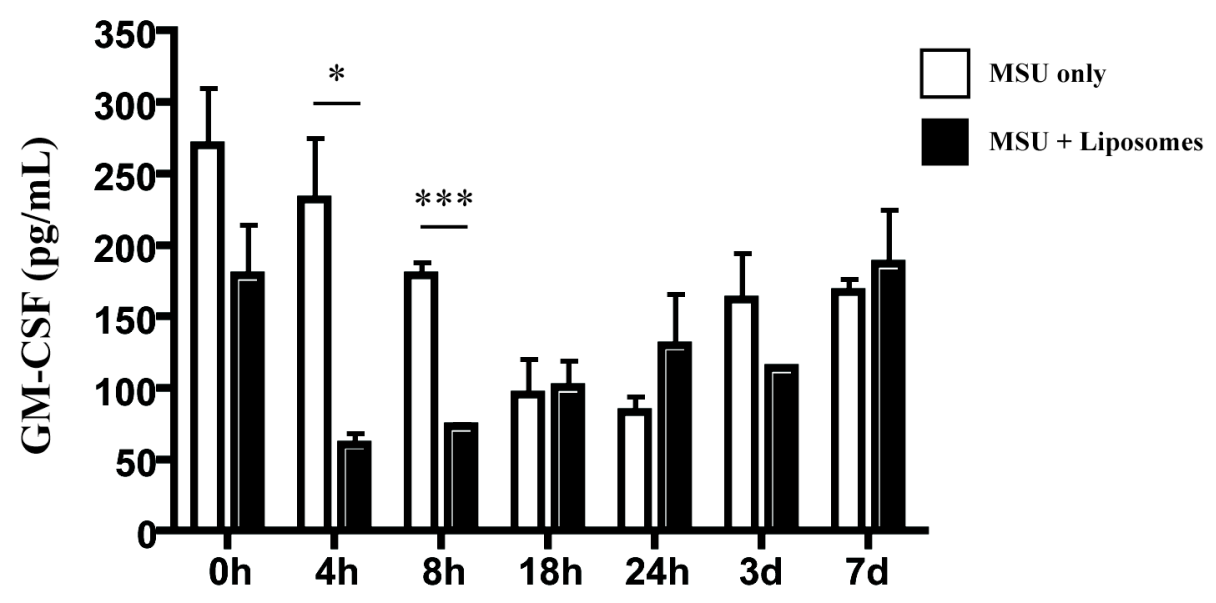

Timepoints

Figure 3.20: Granulocyte-Macrophage-Colony Stimulating Factor levels in macrophage-depleted/non-depleted mice during MSU-induced inflammation. C57B1/6J mice were either left untreated or administered with an i.p. injection of $200 \mu \mathrm{L}$ clodronate liposomes. After three days, mice were injected i.p. with MSU crystals $(3 \mathrm{mg} / 0.5 \mathrm{~mL})$. At different time-points, peritoneal lavages were performed and samples centrifuged. Supernatants from each sample were collected and levels of M-CSF determined by ELISA. Plates were read after 25 minutes. Values represent mean \pm S.E.M. $*=P$ $<0.05 * *=P<0.005$ as determined by two tailed unpaired Student t-test. Results are representative of one experiment with four mice per group. ELISA was conducted by Dr Mischa Walton.

These results indicated that the depletion of resident macrophages significantly altered the profiles of CSFs, which may have contributed to the observed patterns of monocyte differentiation. Macrophage-depletion not only abrogated the inflammatory environment during MSU-induced inflammation, but also increased the background levels of M-CSF while significantly decreasing GM-CSF levels during the acute phase of inflammation.

\subsubsection{Infiltrating neutrophils contribute to inflammation in MSU- treated mice}

As shown in section 3.2.3, the depletion of resident macrophages also significantly inhibited neutrophil infiltration after MSU stimulation in vivo. Neutrophils play a key role in establishing the acute inflammatory microenvironment during the early stages of MSUinduced inflammation by potentiating further neutrophil recruitment through IL-8 production (87) and decreasing inflammation by inducing TGF- $\beta$ production from phagocytes after their apoptosis (142). Therefore it was possible that the absence of 
infiltrating neutrophils in macrophage-depleted mice contributed to delayed MSUrecruited monocyte differentiation and function.

To determine the effect of neutrophils on recruited monocyte function, MSU-recruited cells were harvested from mice eight hours and one week after i.p. MSU administration. PECs were either left as a heterogenous population or the recruited monocytes purified using a density gradient. Both the mixed cell and monocyte preparations were then restimulated with either MSU crystals or LPS and incubated for eight hours (as described in section 3.2.8). Supernatants from the different cell culture systems were analysed for levels of IL-1 $\beta$, IL-6, MCP-1 and KC by ELISA and Bioplex bead array. Cytokine levels were then normalized to the number of monocytes present in each culture, as calculated by flow cytometry analysis ( $\%$ of $\mathrm{F} 4 / 80^{\text {int }} \mathrm{Gr}-1^{+} \mathrm{x}$ total cell number).

Eight hours after MSU administration in vivo, purified monocytes restimulated with MSU crystals produced higher amounts of IL- $1 \beta$ but not IL- 6 compared to the mixed cell population (Figure 3.21A and Figure 3.21B). One week after MSU administration in vivo, (purified recruited-monocytes/macrophages) co-cultured with MSU crystals produced higher levels of pro-inflammatory cytokines IL-1 $\beta$ and IL-6 compared to the mixed cell population (Figure 3.21A and Figure 3.21B). However, it is important to note that aneweek background levels of IL-6 also increased, possibly due to non-specific activation resulting from the isolation process.

Interestingly, after LPS restimulation, purified monocytes produced significantly higher levels of IL-1 $\beta$ compared to mixed cell cultures following re-exposure to MSU crystals (Figure 3.21A). Also, while recruited monocytes isolated at eight hours after MSU administration produced increased levels of IL-6, both purified and mixed cell populations isolated one week after MSU administration produced similar levels of IL-16 after LPS restimulation ex vivo (Figure 3.21B). 


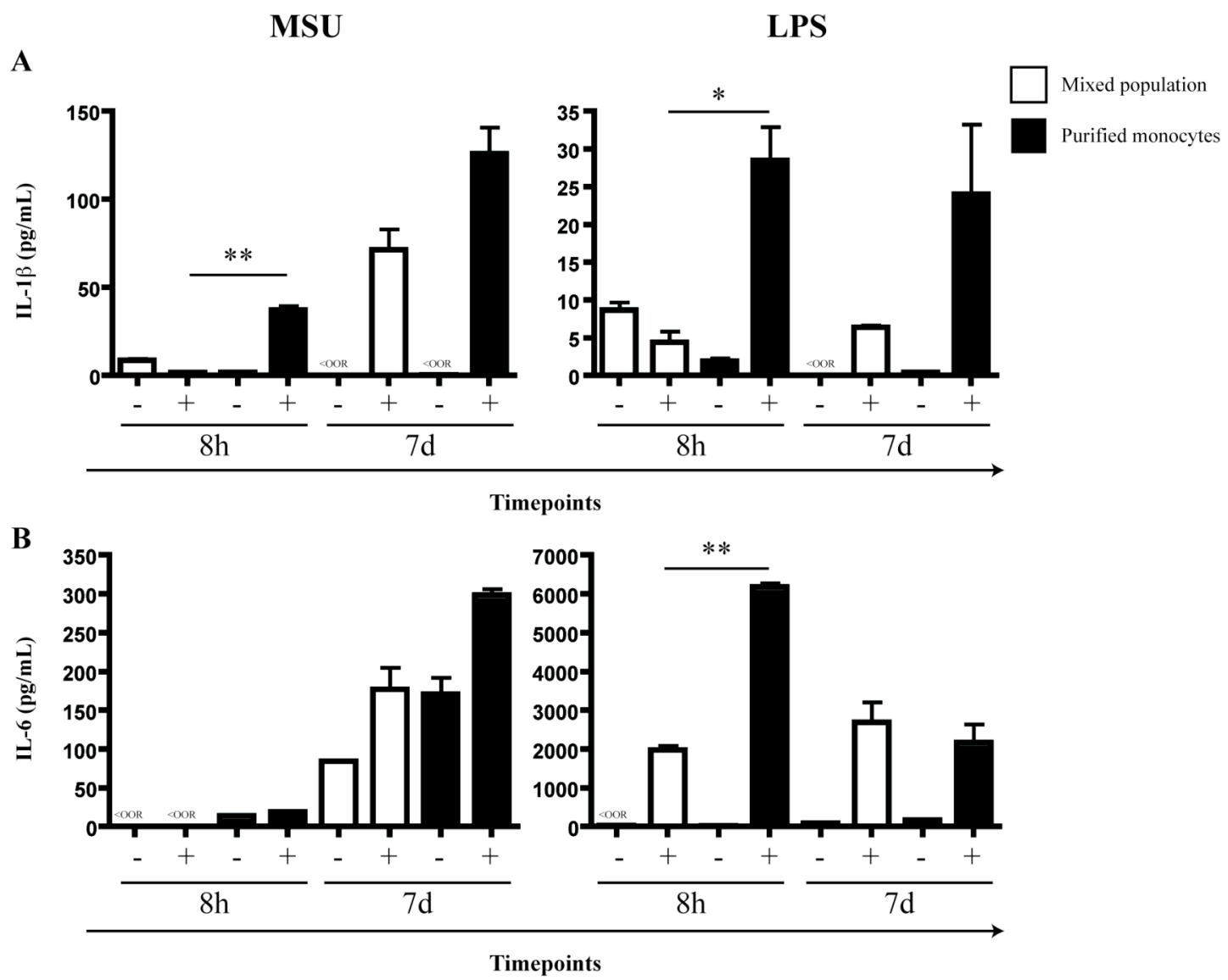

Figure 3.21: Co-incubation of neutrophils abrogates monocyte production of pro-

inflammatory cytokines. C57Bl/6J mice were administered with an i.p. injection of $200 \mu \mathrm{L}$ clodronate liposomes. After three days, mice were injected i.p. with MSU crystals $(3 \mathrm{mg} / 0.5 \mathrm{~mL})$ for eight hours and one week. Peritoneal exudate cells were harvested and either left in a heterogenous population or purified by Lympholyte ${ }^{\circledR}-\mathrm{M}$ density gradient ( $>75 \%$ monocytes). Cells were resuspended at $5 \times 10^{5}$ cells $/ \mathrm{mL}$ in cRPMI and $200 \mu \mathrm{L}$ was cultured in the presence of MSU $(500 \mu \mathrm{g} / \mathrm{mL})$ or LPS $(100 \mathrm{ng} / \mathrm{mL})$ for eight hours. After the incubation period, levels of IL-1 $\beta$ and IL- 6 were determined by ELISA or Bioplex bead array. Cytokine levels were then normalized for the percentage of monocytes presented within the cultures, determined from flow cytometry analysis. Measurements performed in duplicate. Values represent mean \pm S.E.M. $*=P<$ $0.05 * *=P<0.005$ as determined by one-way ANOVA and Tukey multiple comparison test. Results are representative of two independent experiments with five mice per group.

Chemokine levels were also determined by Bioplex bead array from supernatants obtained from the above assays. 
Recruited monocytes purified eight hours after MSU administration increased their capacity to produce both MCP-1 and KC after MSU restimulation compared to mixed cell cultures (Figure 3.22A and Figure 3.22B). There were no significant differences between the purified and mixed cell cultures harvested one week after MSU stimulation in vivo in the production of either MCP-1 or KC (Figure 3.22A and Figure 3.22B). It should be noted that background levels of MCP-1 detected were quite high at both the eight and one week time-points.

Restimulation of purified monocytes harvested eight hours post MSU administration in vivo showed increased production of MCP-1 and $\mathrm{KC}$ compared to the mixed cell cultures (Figure 3.22A and Figure 3.22B). However, there were no significant differences in MCP1 or $\mathrm{KC}$ production between either the purified or mixed populations harvested one week after MSU-administration (Figure 3.22A and Figure 3.22B). 

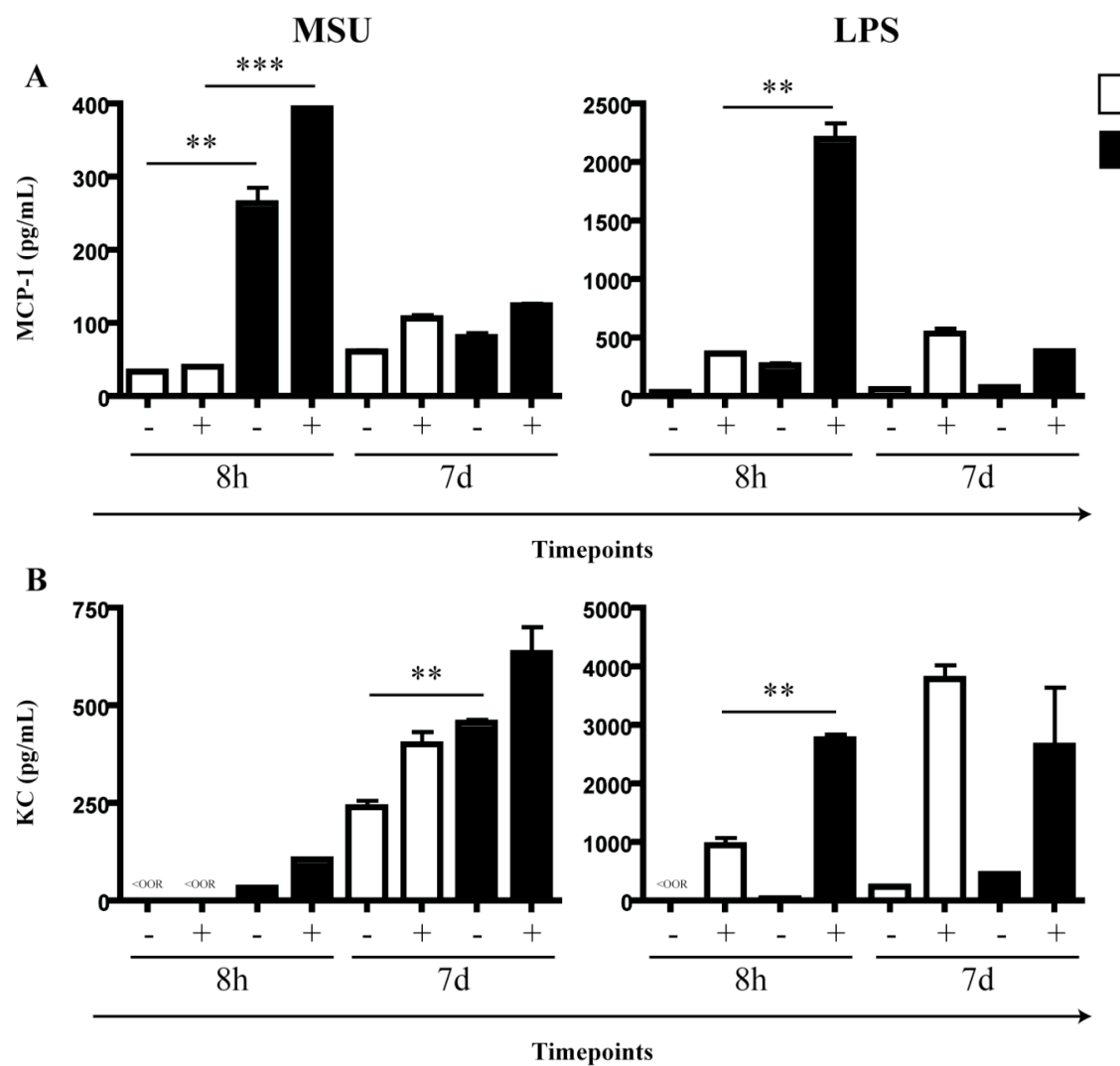

Figure 3.22: Co-incubation of neutrophils abrogates monocyte production of pro-

inflammatory chemokines. $\mathrm{C} 57 \mathrm{Bl} / 6 \mathrm{~J}$ mice were administered with an i.p. injection of $200 \mu \mathrm{L}$ clodronate liposomes. After three days, mice were injected i.p. with MSU crystals $(3 \mathrm{mg} / 0.5 \mathrm{~mL})$ for eight hours and one week. Peritoneal exudate cells were harvested and either left in a heterogenous population or purified by Lympholyte ${ }^{\circledR}-\mathrm{M}$ density gradient ( $>75 \%$ monocytes). Cells were resuspended at $5 \times 10^{5}$ cells $/ \mathrm{mL}$ in cRPMI and $200 \mu \mathrm{L}$ was cultured in the presence of MSU $(500 \mu \mathrm{g} / \mathrm{mL})$ or LPS $(100 \mathrm{ng} / \mathrm{mL})$ for eight hours. After the incubation period, levels of MCP-1 and KC were determined by Bioplex bead array. Cytokine levels were then normalized for the percentage of monocytes presented within the cultures, determined from flow cytometry analysis. Measurements performed in duplicate. Values represent mean \pm S.E.M. ${ }^{* *}=P<$ $0.005 * * *=P<0.0005$ as determined by one-way ANOVA and Tukey multiple comparison test. Results are representative of two independent experiments with five mice per group.

In summary, these results suggested that infiltrating neutrophils might exert a suppressive effect on the pro-inflammatory abilities of recruited monocytes during MSU-induced inflammation. Whether this phenomenon was the result of neutrophil phagocytosis or a soluble factor secreted by neutrophils was then investigated. 


\subsubsection{Soluble factors produced by infiltrating neutrophils suppress recruited-monocyte activity}

Previous studies have shown that phagocytosis of apoptotic neutrophils is a common mechanism of resolution in acute inflammation $(143,152)$. Therefore, uptake of apoptotic neutrophils by MSU-recruited monocytes could have been the reason for the lower proinflammatory cytokines/chemokines detected in mixed cell cultures. However, infiltrating neutrophils have also been shown to produce soluble factors that can influence mononuclear phagocyte function (153) and these secreted molecules could exert a suppressive effect on recruited monocytes.

To determine whether neutrophil secreted soluble factors were responsible for the decreased cytokine/chemokine production in mixed cell populations compared to purified monocytes following MSU restimulation, the effects of different conditioned supernatants from purified neutrophil cultures on isolated recruited-monocytes restimulated with MSU crystals were investigated.

Neutrophils were purified by running PECs harvested from mice administered with MSU i.p. for 18 hours through a density gradient and collecting the resultant cell pellet $(>80 \%$ neutrophils). The purified neutrophils were divided and either apoptosed (cultured in serum free RPMI), activated (cultured with $500 \mu \mathrm{g} / \mathrm{mL}$ MSU crystals in cRPMI), or left untreated (cultured in cRPMI) and incubated for eight hours. The conditioned neutrophil culture supernatants were then stored at $-20^{\circ} \mathrm{C}$ until further use.

$\mathrm{C} 57 \mathrm{Bl} / 6 \mathrm{~J}$ mice were given an i.p. injection of MSU crystals and left for eight hours and one week. MSU-recruited monocytes were purified using Lympholyte ${ }^{\circledR}-\mathrm{M}$ density gradient. The purified monocytes were then resuspended in either cRPMI or the conditioned supernatants from apoptotic or activated neutrophils. The purified monocytes were then restimulated with either MSU crystals $(500 \mu \mathrm{g} / \mathrm{mL})$ or LPS $(100 \mathrm{ng} / \mathrm{mL})$ ex vivo and incubated for eight hours. Supernatants from the co-cultures were analysed for levels of IL-1 $\beta$, IL-6, MCP-1 and KC by ELISA and/or Bioplex bead array. Cytokines were then normalized to the number of monocytes present within the cultures (described in section 3.2.11). 
As shown in Figure 3.23A and Figure 3.23B, early recruited monocytes did not show any differences in their production of IL-1 $\beta$ or IL- 6 when in the presence of neutrophil conditioned supernatants. However, exposure to neutrophil supernatants significantly decreased IL-1 $\beta$ and IL-6 production by purified MSU-recruited monocytes harvested oneweek post MSU-stimulation.

LPS restimulation elicited increased IL-1 $\beta$ and IL-6 levels from purified monocytes compared to unstimulated control (Figure 3.23A and Figure 3.23B). However, there were no significant differences from purified monocyte populations co-incubated with neutrophil-conditioned medium and restimulated with LPS compared with monocytes in cRPMI alone (Figure 3.23A and Figure 3.23B). 


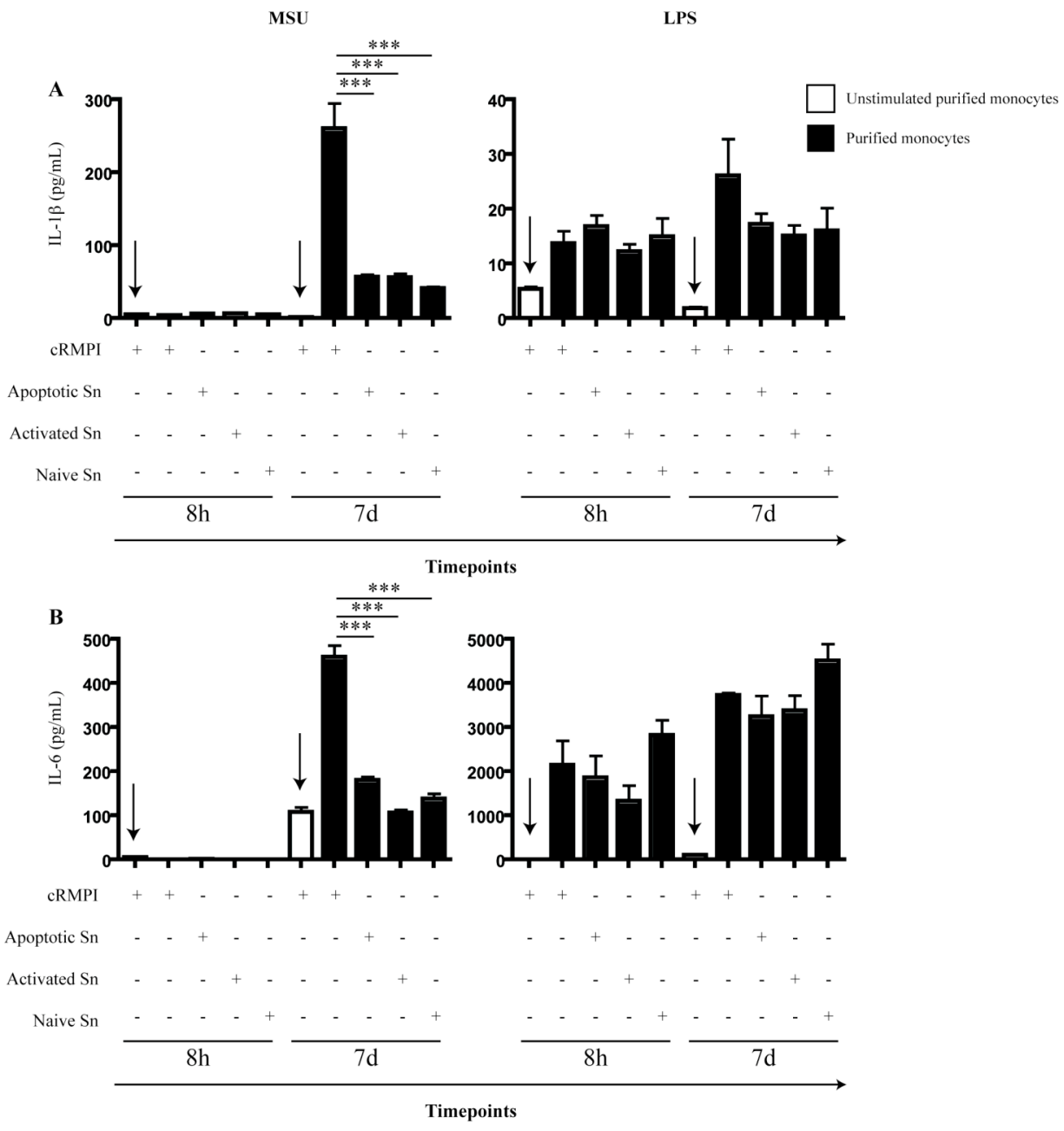

Figure 3.23: Pro-inflammatory cytokine production by restimulated monocytes is abrogated by co-incubation with neutrophil-conditioned medium. C57B1/6J mice were administered with an i.p. injection of MSU crystals $(3 \mathrm{mg} / 0.5 \mathrm{~mL})$ for 18 hours. Peritoneal exudate cells were harvested and purified by Lympholyte ${ }^{\circledR}-\mathrm{M}$ density gradient $\left(>80 \%\right.$ neutrophils). Neutrophils $\left(2 \times 10^{6} / 2 \mathrm{~mL}\right)$ were then either apoptosed (incubated with serum free RPMI), activated (incubated with $500 \mu \mathrm{g} / \mathrm{mL}$ MSU crystals in cRPMI), or left nave (incubated in cRPMI) for eight hours. The supernatants were then collected and stored at $-20^{\circ} \mathrm{C}$. Another group of $\mathrm{C} 57 \mathrm{Bl} / 6 \mathrm{~J}$ mice were then administered with an i.p. injection of MSU crystals $(3 \mathrm{mg} / 0.5 \mathrm{~mL})$ for eight hours and one week. Peritoneal exudate cells were harvested and either left in a heterogenous population or purified by Lympholyte ${ }^{\circledR}-\mathrm{M}$ density gradient $(>75 \%$ monocytes). Cells were resuspended at $5 \times 10^{5}$ cells $/ \mathrm{mL}$ in either cRPMI or prepared neutrophil supernatant and $200 \mu \mathrm{L}$ was cultured in the presence of MSU $(500 \mu \mathrm{g} / \mathrm{mL})$ or LPS $(100 \mathrm{ng} / \mathrm{mL})$ for eight hours. After the incubation period, levels of IL-1 $\beta$ and IL- 6 were determined by ELISA or Bioplex bead array. Cytokine levels were then normalized for the percentage of monocytes present within the cultures determined from flow cytometry analysis. Measurements performed in triplicate. Arrows represent unstimulated control. Values represent mean \pm S.E.M. $* * *=P<0.0001$ as determined by one-way ANOVA and Tukey multiple comparison test. Results are representative of one experiment with ten mice per group. 
Co-incubation of purified monocytes with neutrophil-conditioned supernatants significantly decreased MCP-1 and KC levels detected in culture one week after MSUinduced inflammation (Figure 3.24A and Figure 3.24B).

Whilst LPS restimulation induced increases in $\mathrm{MCP}-1$ and $\mathrm{KC}$ production from purified monocytes compared to unstimulated control (Figure 3.24A and Figure 3.24B), there were no significant differences in MCP-1 or KC production from monocytes co-incubated with neutrophil-conditioned medium and restimulated with LPS compared with monocytes in cRMPI alone (Figure 3.24A and Figure 3.24B). 

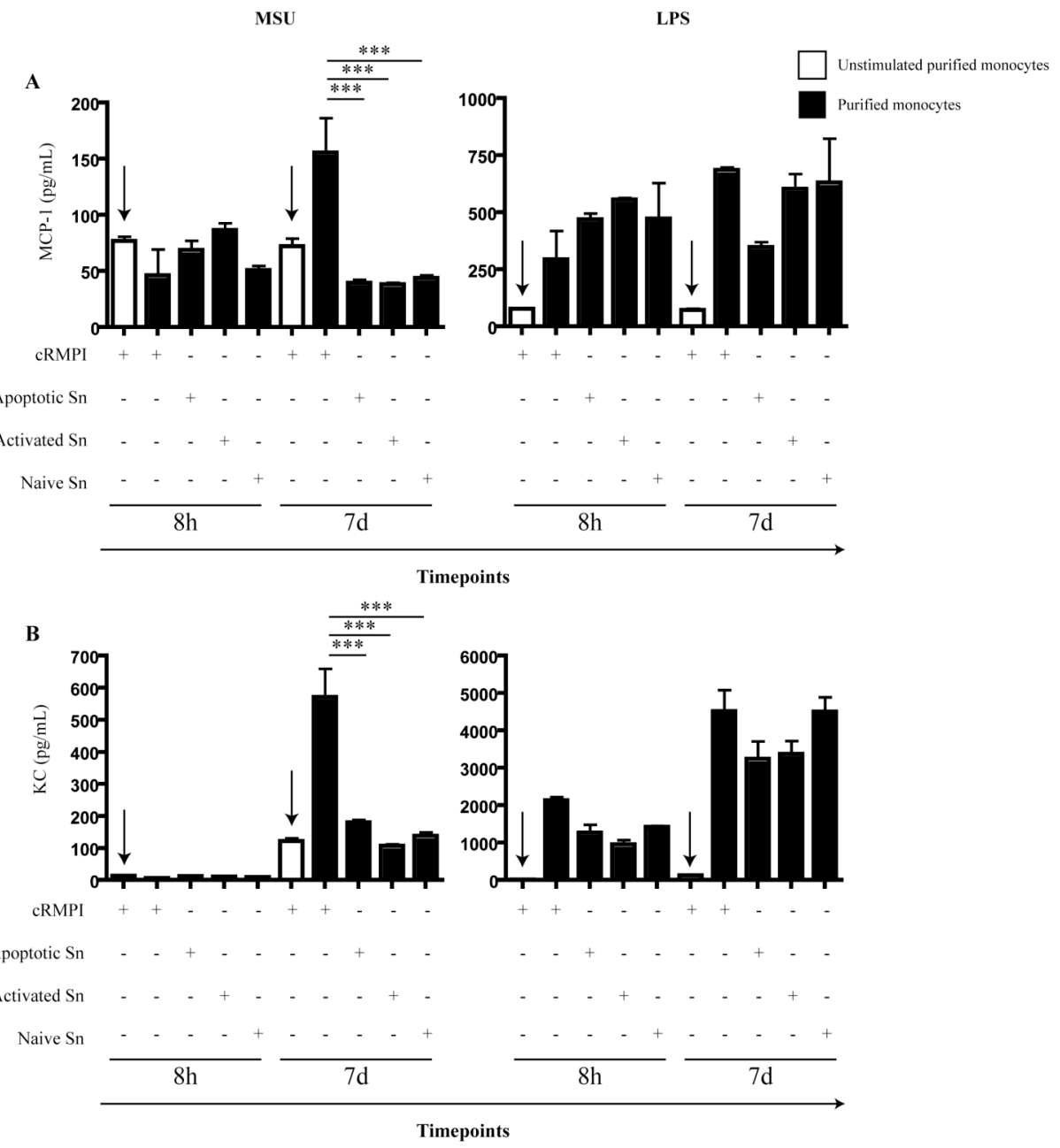

Figure 3.24: Pro-inflammatory chemokine production by restimulated monocytes is abrogated by co-incubation with neutrophil-conditioned medium. C57B1/6J mice were administered with an i.p. injection of MSU crystals $(3 \mathrm{mg} / 0.5 \mathrm{~mL})$ for 18 hours. Peritoneal exudate cells were harvested and purified by Lympholyte ${ }^{\circledR}-\mathrm{M}$ density gradient $\left(>80 \%\right.$ neutrophils). Neutrophils $\left(2 \times 10^{6} / 2 \mathrm{~mL}\right)$ were then either apoptosed (incubated with serum free RPMI), activated (incubated with $500 \mu \mathrm{g} / \mathrm{mL}$ MSU crystals in cRPMI), or left nave (incubated in cRPMI) for eight hours. The supernatants were then collected and stored at $-20^{\circ} \mathrm{C}$. Another group of $\mathrm{C} 57 \mathrm{~B} 1 / 6 \mathrm{~J}$ mice were then administered with an i.p. injection of MSU crystals $(3 \mathrm{mg} / 0.5 \mathrm{~mL})$ for eight hours and one week. Peritoneal exudate cells were harvested and either left in a heterogenous population or purified by Lympholyte ${ }^{\circledR}-\mathrm{M}$ density gradient $(>75 \%$ monocytes). Cells were resuspended at $5 \times 10^{5}$ cells $/ \mathrm{mL}$ in either cRPMI or prepared neutrophil supernatant and $200 \mu \mathrm{L}$ was cultured in the presence of MSU $(500 \mu \mathrm{g} / \mathrm{mL})$ or LPS $(100 \mathrm{ng} / \mathrm{mL})$ for eight hours. After the incubation period, levels of MCP-1 and KC were determined by Bioplex bead array. Cytokine levels were then normalized for the percentage of monocytes presented within the cultures, determined from flow cytometry analysis. Measurements performed in triplicate. Values represent mean \pm S.E.M. $* * *=P<0.0001$ as determined by one-way ANOVA and Tukey multiple comparison test. Results are representative of one experiment with ten mice per group. 
These results indicated that neutrophil-secreted soluble factors might play a role in the suppression of pro-inflammatory cytokines/chemokines produced by MSU-recruited monocytes. However, whether one specific agent or a combination of molecules within the conditioned supernatants mediated the observed suppression was unclear.

\subsubsection{TGF- $\beta$ secreted by infiltrating neutrophils may play a role in suppressing recruited monocyte activity}

TGF- $\beta$ is a powerful pleiotropic anti-inflammatory cytokine. Although the cause of resolution in gout still remains unclear, some studies have suggested a role played for TGF- $\beta$ (142). Neutrophils have been shown to release a wide range of soluble molecules, including superoxide, HBP proteins and small amounts of TGF- $\beta$ (153).

To determine if blocking TGF- $\beta$ would affect monocyte/macrophage production of proinflammatory cytokines/chemokines in response to MSU stimulation, mice were administered i.p. with MSU crystals $(3 \mathrm{mg} / 0.5 \mathrm{~mL})$ for four hours and one week and the recruited PECs collected. Harvested PECs were left in a heterogenous population in cRPMI and restimulated with either MSU crystals or LPS in the presence or absence of $\alpha$ TGF- $\beta$ blocking antibody and incubated for eight hours. Supernatants from the cocultures were analysed for IL-1 $\beta$, IL-6, MCP-1 and KC by ELISA and Bioplex bead array.

Early (4-hours) infiltrating PECs did not produce IL-1 $\beta$ or IL-6 in response to MSU restimulation with or without the TGF- $\beta$ blockade (Figure 3.25A and Figure 3.25B). However, IL-1 $\beta$ and IL-6 production were increased in the one-week mixed cell cultures after MSU restimulation in the presence of TGF- $\beta$ blocking antibody (Figure 3.25A and $3.25 B)$.

There were no statistically significant differences between levels of IL-1 $\beta$ and IL- 6 with or without the blockade of TGF- $\beta$ produced by either early or later-stage MSU-recruited leukocytes after LPS restimulation (Figure 3.25A and Figure 3.25B). Interestingly, in the absence of stimulating agents, TGF- $\beta$ antibody treatment alone resulted in elevated IL- 6 but not IL-1 $\beta$ production. 

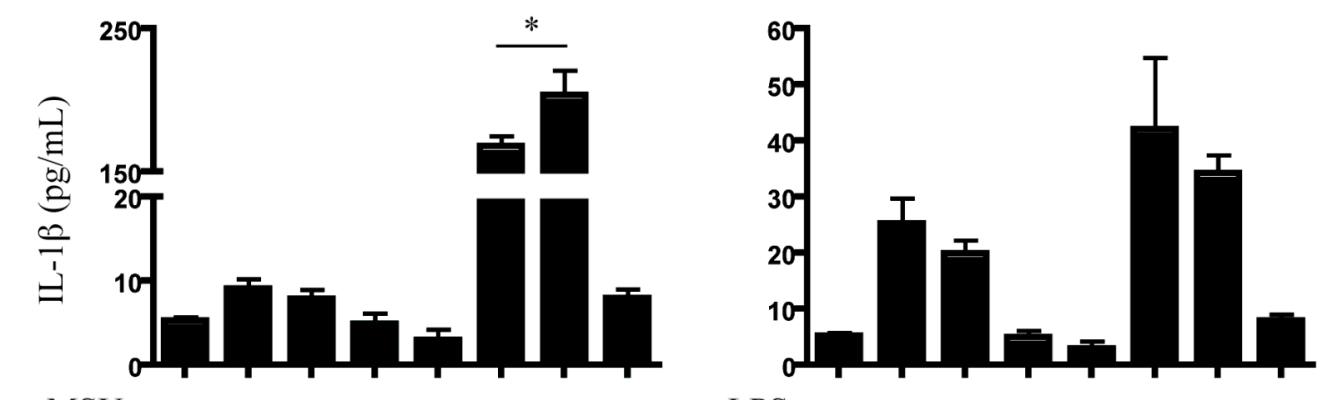

MSU

LPS
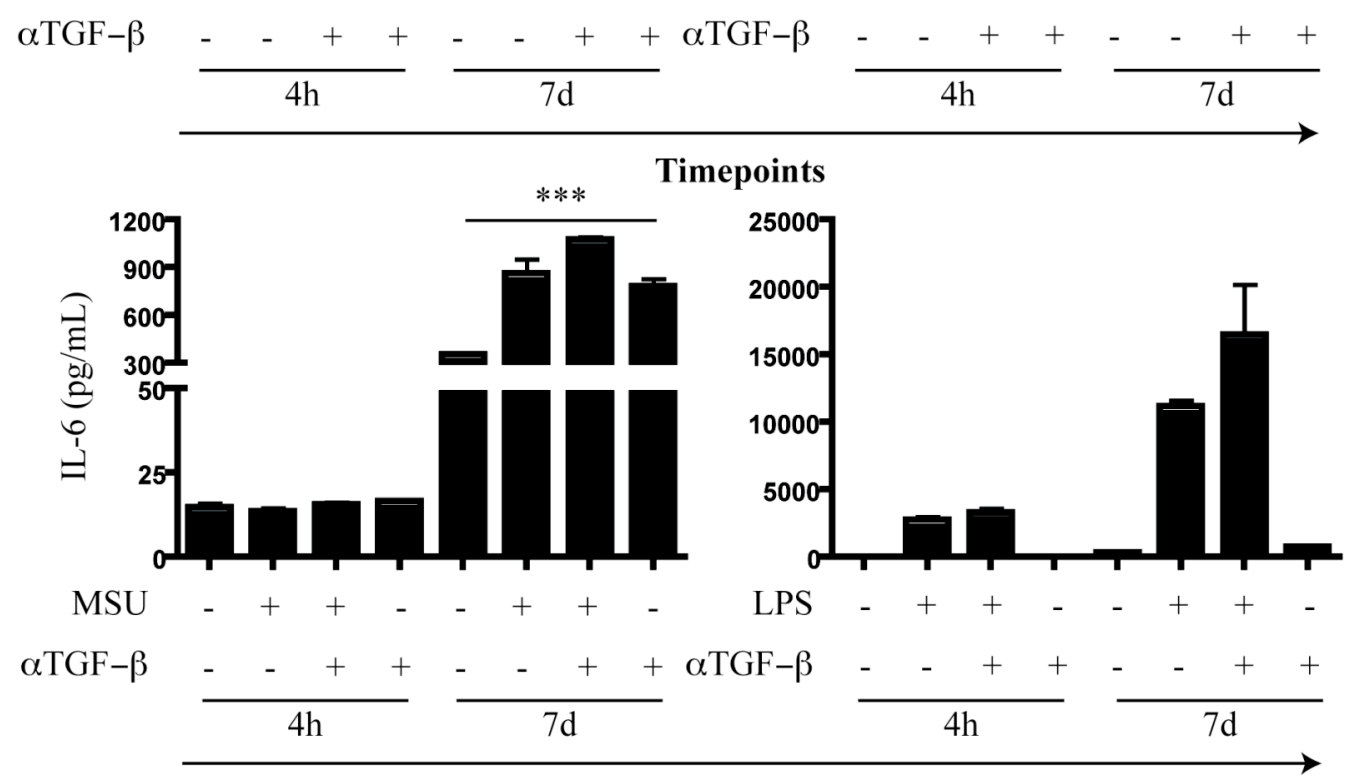

Timepoints

Figure 3.25: TGF- $\beta$ suppresses pro-inflammatory cytokine production by recruited monocytes during later stages of MSU-induced inflammation only. C57Bl/6 $\mathrm{J}$ mice were administered with an i.p. injection of MSU crystals $(3 \mathrm{mg} / 0.5 \mathrm{~mL})$ for four hours and one week. Peritoneal exudate cells were resuspended at $5 \times 10^{5}$ cells $/ \mathrm{mL}$ in cRPMI $200 \mu \mathrm{L}$ was cultured with or without $\alpha \mathrm{TGF}-\beta$ blocking antibody $(32 \mu \mathrm{g} / \mathrm{mL})$ in the presence of MSU $(500 \mu \mathrm{g} / \mathrm{mL})$ or LPS $(100 \mathrm{ng} / \mathrm{mL})$ for eight hours. After the incubation period, levels of IL-1 $\beta$ and IL- 6 were determined by ELISA or Bioplex bead array. Measurements performed in triplicate. Values represent mean \pm S.E.M. $\quad *=P<0.05 * * *=P<0.0001$ as determined by one-way ANOVA and Tukey multiple comparison test. Results are representative of one experiment with ten mice per group. 
Blocking TGF- $\beta$ did not affect the levels of pro-inflammatory chemokines produced after MSU crystal restimulation by mixed recruited leukocyte cultures harvested at the early stages of MSU-induced inflammation (Figure 3.26A and Figure 3.26B). However in later stages, blockade of TGF- $\beta$ significantly increased the levels of MCP-1 and KC produced after restimulation with MSU (Figure 3.26A and Figure 3.26B).

As shown in Figure 3.26A and Figure 3.26B, mixed cell cultures harvested during the early stages of MSU-induced inflammation incubated with TGF- $\beta$ and restimulated with LPS produced similar levels of MCP-1 and KC. While the former was unaffected with TGF- $\beta$ blockade (Figure 3.26A), production of KC was significantly increased after LPS restimulation (Figure 3.26B). 


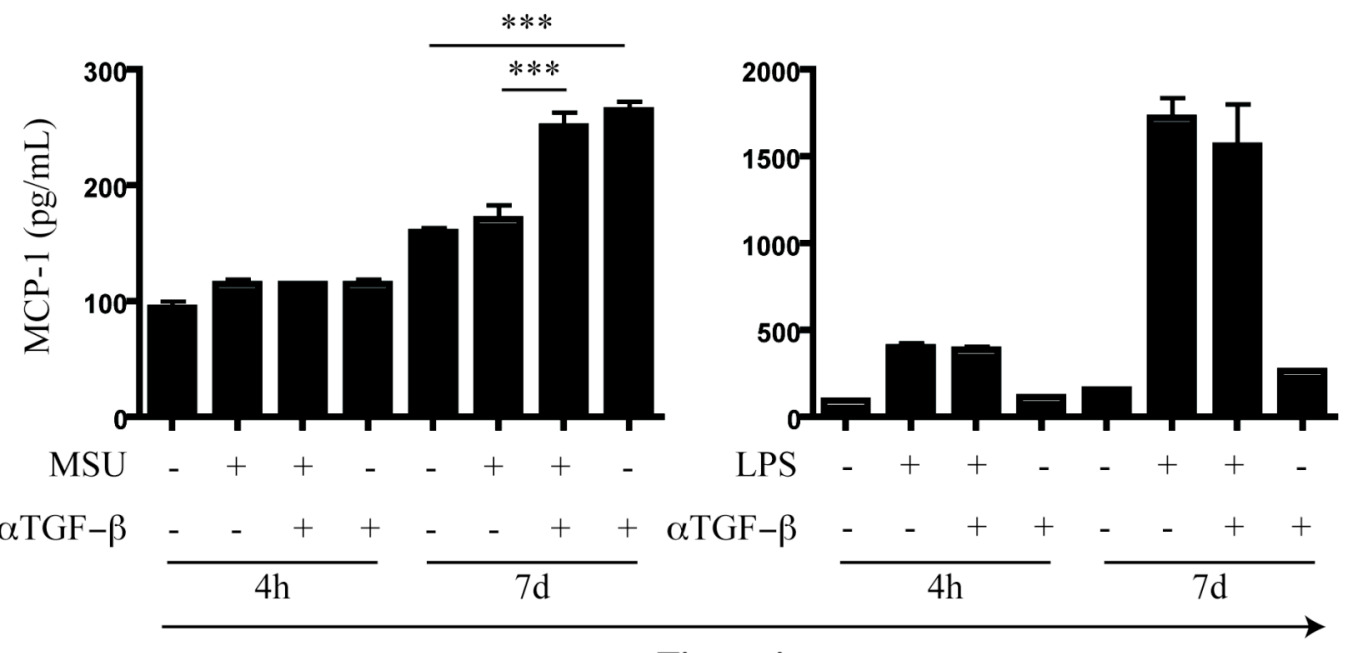

Timepoints

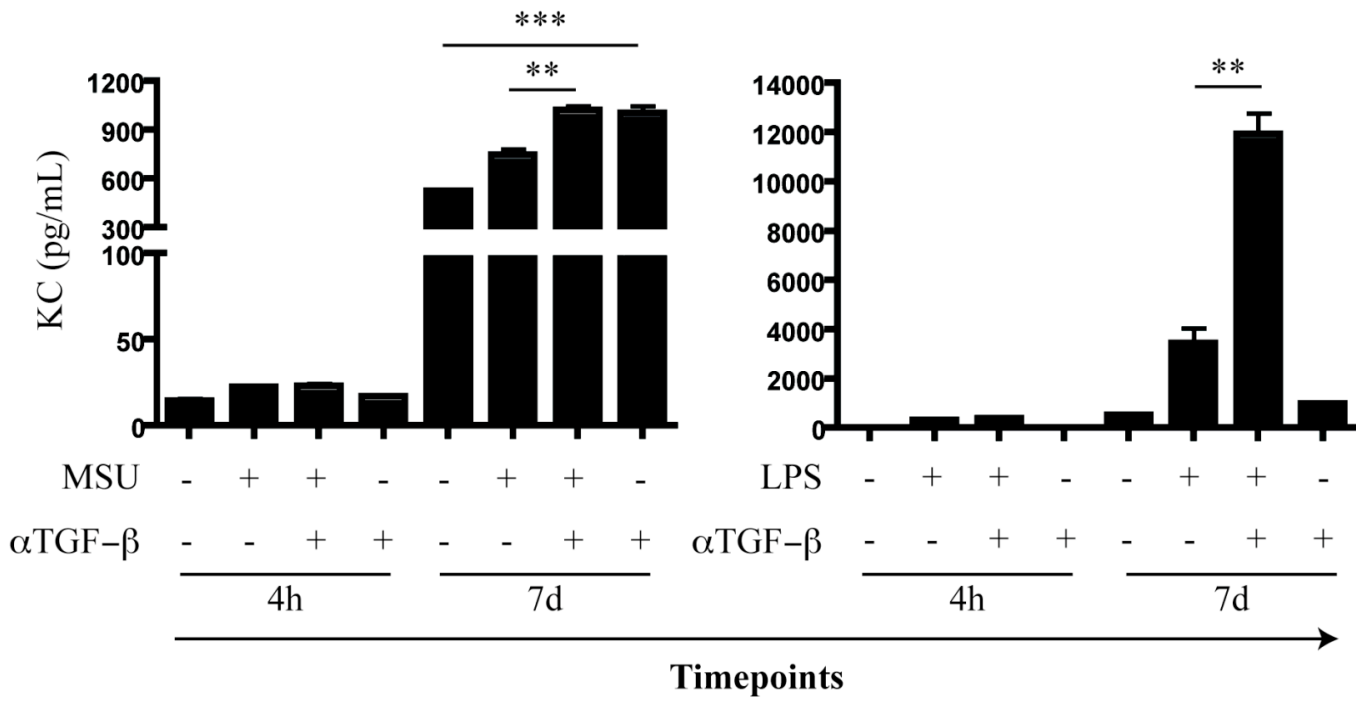

Figure 3.26: TGF- $\beta$ suppresses pro-inflammatory chemokine production by recruited monocytes during later stages of MSU-induced inflammation only. C57B1/6J mice were administered with an i.p. injection of MSU crystals $(3 \mathrm{mg} / 0.5 \mathrm{~mL})$ for four hours and one week. Peritoneal exudate cells were resuspended at $5 \times 10^{5}$ cells $/ \mathrm{mL}$ in cRPMI $200 \mu \mathrm{L}$ was cultured with or without $\alpha$ TGF- $\beta$ blocking antibody $(32 \mu \mathrm{g} / \mathrm{mL})$ in the presence of MSU $(500 \mu \mathrm{g} / \mathrm{mL})$ or LPS $(100 \mathrm{ng} / \mathrm{mL})$ for eight hours. After the incubation period, levels of MCP-1 and $\mathrm{KC}$ were determined by Bioplex bead array. Measurements performed in triplicate. Values represent mean \pm S.E.M. $* *=P<0.001 * * *=P<0.0001$ as determined by one-way ANOVA and Tukey multiple comparison test. Results are representative of one experiment with ten mice per group. 
These results indicated that the anti-inflammatory cytokine TGF- $\beta$ did not affect the production of pro-inflammatory molecules by early-recruited leukocytes after MSU or LPS restimulation. However, blocking TGF- $\beta$ and restimulating with MSU increased the levels of IL-1 $\beta, \mathrm{MCP}-1$ and $\mathrm{KC}$ produced by leukocytes harvested at the later stages of the inflammatory response. While restimulation with LPS in the presence of $\alpha$ TGF- $\beta$ antibody induced an increase in KC production by PECs, blocking TGF- $\beta$ did not affect IL-1 $\beta$, IL-6 or MCP-1 production.

\subsection{Discussion}

MSU-induced peritoneal inflammation is a commonly used murine model of the acute and highly pro-inflammatory environment that occurs during a gout attack. Mononuclear phagocytes are highly plastic cells capable of differentiating into various subsets with completely different roles depending on the programming signals. The findings presented within this chapter give insight into the impact of resident macrophages, infiltrating neutrophils, and the inflammatory microenvironment in general on the differentiation and function of MSU-recruited monocytes.

\subsubsection{MSU-induced monocyte recruitment in normal and macrophage-depleted mice}

The depletion of resident macrophages decreased the levels of monocyte chemoattractant MCP-1 detected within the peritoneum during early inflammation after MSU administration (section 3.2.4). However, the low MCP-1 levels did not correspond with a decrease in the number of monocytes recruited into the local environment (section 3.2.3). This may be attributed to a MCP-1 threshold which once reached allows for optimum monocyte infiltration into the peritoneal cavity. Alternatively, another mediator (such as MIPs $(154)$ or M-CSF $(155,156))$ other than MCP-1 is produced by resident cells after exposure to MSU crystals and is also responsible for the recruitment of monocytes from the circulation during acute inflammation. 


\subsubsection{Effect of the inflammatory environment on monocyte differentiation and function}

Although macrophage depletion abrogated IL-1 $\beta$ and IL-6 production within the MSUinduced pro-inflammatory environment (section 3.2.4), TNF $\alpha$ levels remained unaffected. This could be attributed to production by epithelial cells (such as endothelial cells (157) and fibroblasts (158)), which can maintain the production of TNF $\alpha$ upon stimulation.

The inflammatory environment plays a vital role in driving the differentiation of recruited monocytes to macrophages. Morphologically (section 3.2.5), monocytes recruited into a macrophage-depleted environment in response to MSU showed stalled development of physiological phenotypes (such as decreased cytoplasmic volume) compared to MSUrecruited monocytes from normal mice. Interestingly, this delay in differentiation only weakly correlated with changes in the cell surface phenotype. While the downregulation of recruited monocyte hemopoietic markers were notably hampered (section 3.2.7), this was not associated with a delay in the upregulation of macrophage markers (section 3.2.8). Hence, this serves as a caveat on not solely relying on cell surface markers to distinguish differences in cell differentiation, and that fundamental methods, although subjective, can sometimes contribute to the overall picture via more basic and unobstructed observations.

During the early stages (4-hour) of MSU-induced inflammation, monocytes recruited into a macrophage-depleted inflammatory environment produced pro-inflammatory cytokines in response to LPS but not MSU crystal restimulation (section 3.2.8). Production of IL-1 $\beta$ and IL- 6 by monocytes isolated from both normal and macrophage-depleted mice in response to LPS restimulation indicate that the TLR pathways that mediate responses to LPS may only be marginally affected by an abrogation in the acute inflammatory response. Whilst the delayed production of pro-inflammatory cytokines by recruited monocytes from macrophage-depleted mice after MSU crystal restimulation could be due to impaired phagocytic ability (section 3.2.9), Latz and colleagues have shown that the encapsulation of MSU crystals leads to damage and subsequent rupture of the intracellular lysosomes in phagocytes(56). Resulting in NALP3 inflammasome activation and the production of IL$1 \beta$ by caspase- 1 cleavage of pro-IL- $1 \beta(60,159)$. 
Consistent with this, impaired uptake of fluorescent beads was also shown (section 3.2.9). Therefore it appears that the inflammatory environment plays a role in the development of phagocytic function in monocytes, which enables recruited cells to recognize MSU crystals and produce pro-inflammatory cytokines. It is possible that delayed development of phagocytic function observed in MSU-recruited monocytes from macrophage-depleted mice is responsible for decreased stimulation of monocytes via the classical NALP3 inflammasome pathway.

\subsubsection{The impact of colony-stimulating factors on recruited monocyte differentiation}

The depletion of resident macrophages caused an elevation of background levels of MCSF (section 3.2.10). M-CSF has been shown previously to circulate at detectable levels at steady state and have been shown previous to give rise to osteoclast lineage cells in conjunction with RANKL (receptor activation of NFKB ligand) (160). Therefore at baseline levels, M-CSF may play a role in inducing myeloid differentiation, contributing to background tissue growth and healing. The depletion of resident macrophages may trigger secretion of intracellular reservoirs of M-CSF by epithelial resident cells such as fibroblasts and endothelial cells (151). As CSFs have chemotactic properties $(155,156)$, elevated baseline levels of M-CSF may contribute to the recruitment of circulating myeloid precursors designed to replenish the depleted resident macrophage population.

In an abrogated inflammatory environment depleted of macrophages, the levels of M-CSF and GM-CSF were significantly decreased during the early phases of MSU-induced inflammation (section 3.2.10). Previous studies examining lineage-development has shown that GM-CSF and M-CSF can compete with each other to generate different cellular phenotypes when co-cultured with myeloid precursor cells (161). Stimulation of immature macrophage-lineage populations with GM-CSF and IL-4 generate a DC-like phenotype (162-164), whereas stimulation with both M-CSF and GM-CSF results in a more macrophage-like population (161). In this study, the change in levels of both M-CSF and GM-CSF within the inflamed peritoneum of macrophage-depleted mice may have altered programming signals required for efficient monocyte to macrophage differentiation. Therefore future directions may warrant investigation into the ratios of GM-CSF and M- 
CSF required for monocyte/macrophage differentiation, possibly by using knockout mice or antibody depletion. This may give insight into which CSF plays a more predominant role in generating macrophage- or DC-like phenotypes from MSU-recruited monocytes.

\subsubsection{Neutrophil suppression of recruited monocyte pro- inflammatory capacity during MSU-induced inflammation}

During the early stages (8-hours) of MSU-induced inflammation, co-incubation of infiltrating neutrophils with recruited monocytes had an immunosuppressive effect on the pro-inflammatory capabilities of the monocyte population (section 3.2.11). Neutrophil derived products have been previously shown to regulate monocyte/macrophage activity (165). However, as co-incubation of neutrophil-derived soluble factors did not affect the pro-inflammatory capabilities of the early (8-hour) MSU-recruited monocytes (section 3.2.12), it is possible that infiltrating monocytes may drive resolution of acute inflammation via neutrophil uptake and switching to a non-inflammatory phenotype. This finding is consistent with previous studies showing that the uptake of apoptotic neutrophils can downregulate the production of pro-inflammatory cytokines from phagocytosing mononuclear cells in acute inflammation (142). In addition, Terkeltaub and colleagues has shown that macrophage phagocytosis of apoptotic thymocytes prior to MSU administration decreases macrophage responsiveness to stimulation in vivo (166). Although it is a widely accepted phenomenon in macrophages (167), there has been no evidence of the phagocytosis of apoptotic neutrophils switching recruited monocytes from a "pro"- to "non"- inflammatory phenotype.

At the later (1-week) time-point of MSU-induced inflammation, neutrophil-derived soluble factors were able to suppress the production of pro-inflammatory cytokines by purified monocytes/macrophages after MSU but not LPS restimulation (section 3.2.12). It is possible that neutrophil-derived products may limit the inflammatory response by suppressing differentiated monocytes/macrophages from being reactivated by MSU crystals still deposited within the peritoneal cavity. As neutrophils have been previously shown to produce TGF- $\beta$ (168), administering TGF- $\beta$ antibody in conjunction with MSU crystals in the rat air pouch model inhibited the overall acute inflammatory response (110). In this study, adding anti-TGF- $\beta$ blocking antibody to mixed PEC cultures inhibited the 
suppressive effects of neutrophil-secreted TGF- $\beta$ and increased monocyte production of cytokines/chemokines after MSU restimulation (section 3.2.13). Hence, this indicates that neutrophil-derived soluble factors (including TGF- $\beta$ ) can suppress recruited monocyte/macrophage responsiveness to MSU crystal restimulation, which may contribute to the resolution of MSU-induced inflammation.

An interesting finding of this study was that the purification of MSU-recruited monocytes resulted in increased background levels of cytokines/chemokines. This could be possibly due to the isolation of recruited monocytes from a suppressive effect of other cells/molecules (such as neutrophil TGF- $\beta$ ) released from the local environment as an inflammatory regulator. Another possibility for the elevated background levels could be due to non-specific activation of monocytes from the density gradient purification procedure. While the specific cause remains currently unclear, the density gradient purification process is a better method than AutoMACs or cell sorting in providing an isolated monocyte population from PECs while limiting non-specific cellular activation. Nevertheless, these data indicate the importance of investigating cell function in "natural" mixed cell environments. When investigating purified cell populations as the ex vivo mixed population results differ significantly from earlier in vitro data, using isolated cell populations.

In summary, this study showed that depletion of resident macrophages abrogated the inflammatory environment into which monocytes were recruited in response to MSU stimulation. Over the course of the MSU-induced inflammation, recruited monocytes from macrophage-depleted mice displayed delayed differentiation into macrophages. This phenotypic change in differentiation correlated with stalled development of phagocytic function. Despite developing the ability to produce pro-inflammatory cytokines and chemokines in response to LPS, these altered monocytes do not show the same sensitivity to MSU restimulation, indicating that the inflammatory environment can alter the development of MSU recognition pathways independent of TLR signalling. The uptake of neutrophils by recruited monocytes may be the key mechanism in initiating resolution in MSU-induced inflammation. In addition, TGF- $\beta$ may play a role in suppressing the MSUinduced inflammatory response and help maintain homeostatic regulatory control. 
Chapter 4.0:

Recruitment and the effects of Glatiramer acetate on Monocytes in MSU-induced inflammation 


\subsection{Monocyte recruitment by peritoneal mesothelial cells in MSU-induced inflammation}

\subsubsection{Leukocyte migration}

Leukocyte recruitment from the bloodstream to the site of inflammation occurs through a strictly regulated pathway known as the adhesion cascade (described in Chapter 1).

In brief, various selectins (such as L- and E- selectins) on circulating leukocytes interact with selectins (such as PSGL1) and other co-molecules expressed on endothelial cells creating a "rolling" effect along the inner walls of blood vessels (72). While chemokines (such as MCP-1) are responsible for the arrest of the "rolling" leukocytes (71), integrins expressed on circulating cells interact with adhesion molecules along the blood vessels contributing to further strengthening of adhesion prior to transmigration (74).

Leukocytes transmigrate from the bloodstream through the endothelium and into inflamed tissue by three mechanisms; crawling, paracellular and transcellular migration (71). Crawling occurs when "rolling" leukocytes are seeking preferred sites of transmigration (77). Paracellular migration occurs when ligands expressed on the leukocytes interact with adhesion molecules on the endothelium leading to reduced inter-endothelial contacts and allowing migration of cells through the vessel walls (77). Transcellular migration occurs mainly in the thin endothelium within the CNS, whereby cells may transmigrate through intracellular channels within the endothelial cells mediated by various ligand-to-adhesion molecule interactions (78).

\subsubsection{Monocyte recruitment in MSU-induced inflammation}

Early inflammation in acute gout is associated with the infiltration of circulating monocytes into the joint synovium (125). In the MSU-induced model of acute gout, activated macrophages have been identified as a key cell type involved in the recruitment of cells from the bloodstream through the production of monocyte - (MCP-1) and neutrophil - (KC) attracting chemokines (69). Previous studies in our group have shown that the depletion of peritoneal resident macrophages significantly abrogated neutrophil 

induced inflammation

infiltration in MSU-induced inflammation, while monocyte infiltration remained unchanged (58). This indicated that monocyte recruitment in gout could be regulated by a mechanism independent of activated resident macrophages and that other resident cells may orchestrate monocyte recruitment in response to MSU stimulation. 
Preliminary studies in our group have shown that whole peritoneal tissue stimulated with MSU crystals produces the chemokine MCP-1 but not other pro-inflammatory cytokines such as TNF $\alpha$, IL-1 $\beta$ and IL-6 (unpublished data). However, several aspects remain unknown 1) whether this response was specific to MSU crystals 2) which cell type on the peritoneal lining was responsible for MCP-1 production and 3) which pathway(s) might be involved in MSU-induced MCP-1 production in the membrane.

\subsubsection{Objectives}

The aim of this section was to determine whether MCP-1 produced by mesothelial cells could be responsible for monocyte recruitment in response to direct MSU crystal stimulation. To address this question, we investigated the production of MCP-1 by both the peritoneal membrane and cultured mesothelial cells, and whether the TLR4 pathway plays a role.

\subsubsection{Results}

\subsubsection{MCP-1 production by the peritoneal membrane after MSU or LPS stimulation}

To investigate the phenomenon of macrophage independent MSU-induced MCP-1 production, excised peritoneal membranes were recovered from naïve $\mathrm{C} 57 \mathrm{Bl} / 6 \mathrm{~J}$ mice and stimulated with MSU crystals or LPS for 6 hours in vitro. This incubation period correlates with acute MCP-1 production in vivo (58). Separate membrane preparations were also stimulated with MSU crystals or LPS in the presence of an anti-CD14 blocking antibody in order to determine whether the MCP-1 production was dependent on the TLR4 pathway. The culture supernatants were then analysed for MCP-1 levels by Bioplex bead array.

Consistent with previous observations by other members of our group, excised peritoneal tissues produced significant levels of MCP-1 when exposed to MSU crystals (Figure 4.1). This response was also observed after stimulation with LPS. The production of MCP-1 
induced by either MSU crystals or LPS was significantly abrogated following blockade of CD14.

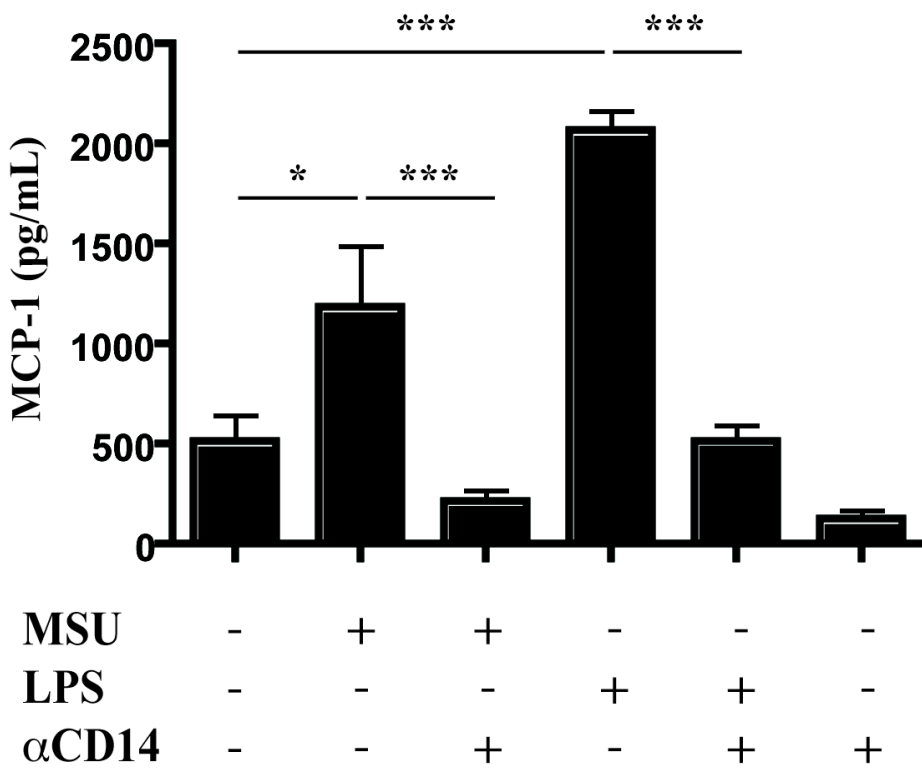

Figure 4.1: MSU-induced MCP-1 production by the peritoneal membrane is mediated by CD14. Peritoneal membranes were excised from naïve C57B1/6J mice and either left untreated or incubated with or without anti-mouse CD14 blocking antibody $(20 \mu \mathrm{g} / \mathrm{mL})$ in the presence of MSU crystals $(500 \mu \mathrm{g} / \mathrm{mL})$ or LPS $(100 \mathrm{ng} / \mathrm{mL})$. After six hours incubation, MCP-1 levels were determined by Bioplex bead array. Measurements performed in triplicate. Values represent mean \pm S.E.M. $*=P<0.05$ *** $=P<0.001$ as determined by one-way ANOVA and Tukey multiple comparison test. Results are representative of two independent experiments.

These results showed that the peritoneal membrane could produce MCP-1 in response to direct MSU stimulation, in the absence of resident macrophages. Therefore, this indicated that the cells lining the peritoneal membrane may be a key source of the chemoattractant MCP-1 responsible for MSU-induced monocyte recruitment.

\subsubsection{Isolation, identification and culture of peritoneal mesothelial cells}

Mesothelial cells are one of the cell types present on peritoneal membranes. The first aim was to stain the whole peritoneal membrane to identify the presence of cytokeratin ${ }^{+}$ mesothelial cells. However, the staining antibodies non-specifically bound to collagen 
fibres covering the membrane resulting in high levels of background fluorescence when analysed microscopically.

As an alternative approach we looked at isolating and culturing mesothelial cells for subsequent in vitro stimulation with MSU crystals. In order to harvest PMCs $(126,127)$, the peritoneums of naïve $\mathrm{C} 57 \mathrm{Bl} / 6 \mathrm{~J}$ mice were first washed with heparin/PBS to remove the resident leukocytes. A second peritoneal lavage was performed using $0.25 \%$ Trypsin/EDTA $(3 \mathrm{~mL})$ and left in the peritoneum for 15 minutes and the exudate cells were collected. The process was repeated for a third time using cRPMI to maximise mesothelial cell collection. Mesothelial cells were then resuspended in cRPMI cultured in vitro for six to eight days in the presence of epithelial growth factor. Cells were identified as mesothelial cells (Figure 4.2) by their large size $(60-80 \mu \mathrm{m}$ in diameter), tendency to form monolayers, and cobblestone appearance $(126,174)$.

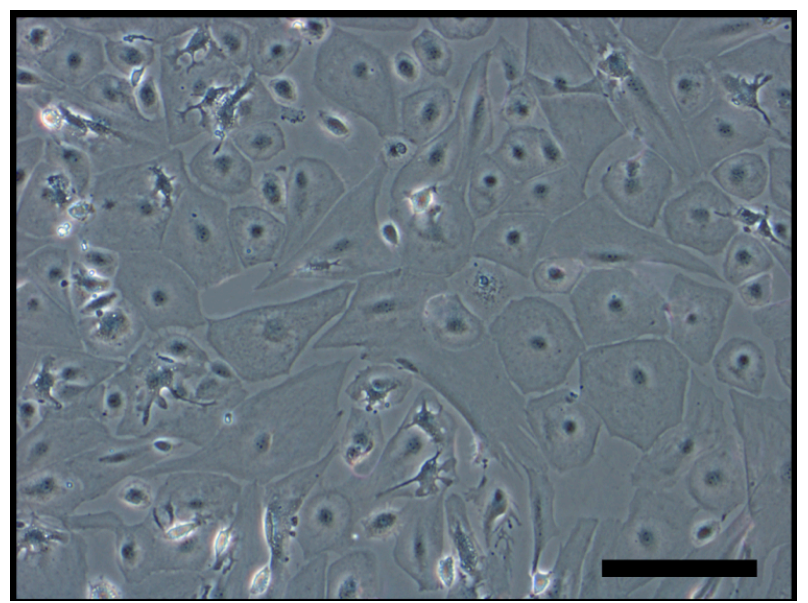

Figure 4.2: Identification of peritoneal mesothelial cells. Peritoneal mesothelial cells were harvested from naïve C57B1/6J mice by treatment with $0.25 \%$ Trypsin/EDTA in vivo and cultured in vitro. A phase contrast picture was taken to identify peritoneal mesothelial cells by the squamous cobble stone appearance. Original magnification 40x. Scale bar represents $100 \mu \mathrm{m}$. Results representative of at least three independent experiments. Figure 4.2 was provided by Dr Willy-John Martin. 
To confirm the production of MCP-1 by mesothelial cells after stimulation with MSU crystals or LPS, cells were stained for the mesothelial cell marker cytokeratin and MCP-1 and analysed by immunofluorescent histology. As shown in Figure 4.3, both MSU and LPS stimulated cytokeratin ${ }^{+}$mesothelial cells to produce MCP-1.
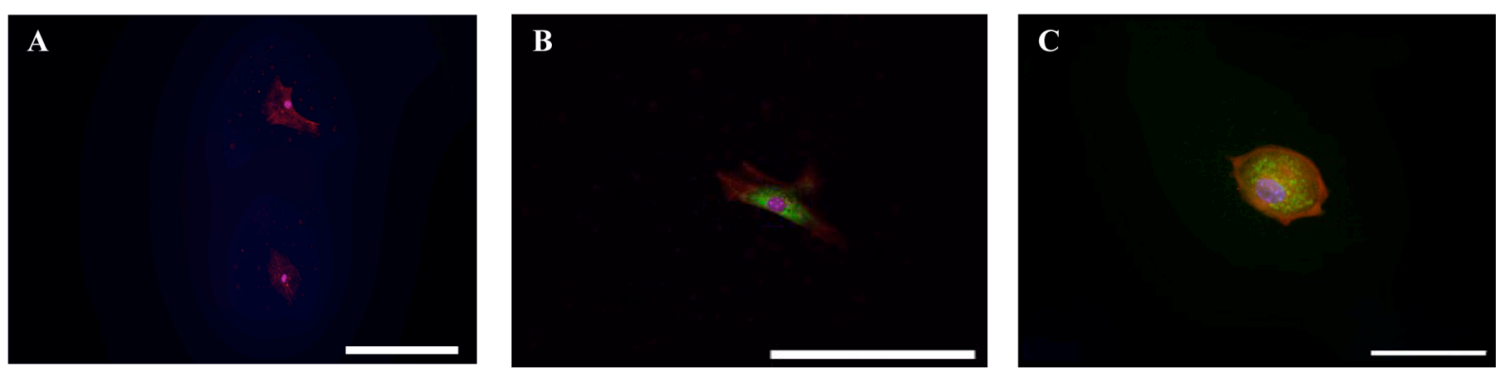

Figure 4.3: MCP-1 production by cultured peritoneal mesothelial cells. Peritoneal mesothelial cells were harvested from naïve C57B1/6J mice by treatment with $0.25 \%$ Trypsin/EDTA in vivo and cultured in vitro. A. Cells were either left untreated or B. exposed to MSU $(500 \mu \mathrm{g} / \mathrm{mL})$ or C. LPS $(100 \mathrm{ng} / \mathrm{mL})$ in the presence of GolgiStop for four hours. Production of MCP-1 (green) by cytokeratin positive (red) peritoneal mesothelial cells was then determined by fluorescent antibody staining. Original magnification 20x, 20x and 40x respectively. Scale bar represents $100 \mu \mathrm{m}$. Results representative of two independent experiments.

\subsubsection{Peritoneal mesothelial cells produce MCP-1 after MSU or LPS stimulation}

To confirm whether CD14 was involved in MSU-induced MCP-1 production by mesothelial cells, cultured mesothelial cells were activated with MSU or LPS in the presence of CD14 blocking antibody, and the MCP-1 levels within the supernatant determined. Consistent with the whole peritoneal tissue experiments, mesothelial cells produced significant levels of MCP-1 after stimulation with either MSU or LPS (Figure 4.4). However, while MCP-1 elicited by LPS was significantly abrogated following CD14 blockade, MCP-1 production after MSU crystal stimulation was unaffected. 


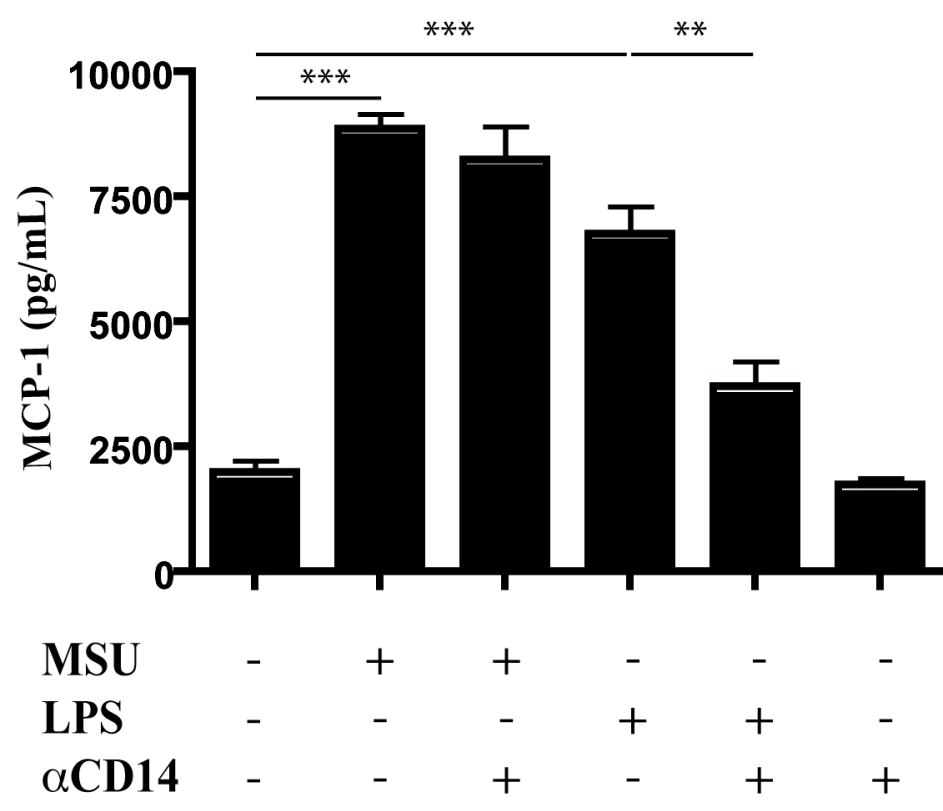

Figure 4.4: MSU-induced MCP-1 production by cultured peritoneal mesothelial cells is not mediated by CD14. Peritoneal mesothelial cells were harvested from naïve $\mathrm{C} 57 \mathrm{Bl} / 6 \mathrm{~J}$ mice by treatment with $0.25 \%$ Trypsin/EDTA in vivo and cultured in vitro. Cells were either left untreated or incubated with or without anti-mouse CD14 blocking antibody $(20 \mu \mathrm{g} / \mathrm{mL})$ in the presence of MSU crystals $(500 \mu \mathrm{g} / \mathrm{mL})$ or LPS $(100 \mathrm{ng} / \mathrm{mL})$. After six hours incubation, MCP-1 levels were determined by Bioplex bead array. Measurements performed in triplicate. Values represent mean \pm S.E.M. $* *=P<0.01 * * *=P$ $<0.001$ as determined by one-way ANOVA and Tukey multiple comparison test. Results are representative of two independent experiments.

\subsubsection{Discussion}

Recruitment of pro-inflammatory leukocytes from the bloodstream into the peritoneum is considered to be an important aspect of inflammation and resolution in gout $(91,113)$. Therefore, it is especially important to understand the mechanisms involved in their recruitment as possible targets to be used in clinical therapy.

Activated macrophages were previously thought to play a role in monocyte recruitment (69) however; studies in our group have shown otherwise. Our observations have shown that while activated resident macrophages play a pivotal role in neutrophil recruitment (58) (also described in chapter 3), another mechanism is responsible for monocyte migration. 
The findings presented within this section have shown that the peritoneal membrane was responsible for MCP-1 production upon stimulation with MSU crystals in vitro and that this process was CD14 dependent. However, while cytokeratin ${ }^{+}$cultured mesothelial cells produced MCP-1 after stimulation with MSU crystals or LPS, CD14 antibodies blocked only the LPS response. The differential responses of PMCs to MSU and LPS in vitro suggests that the CD14-dependent TLR4 pathway does not mediate the recognition of MSU crystals by mesothelial cells. This was surprising in light of the apparent dependence on CD14 for the peritoneal membrane to produce MCP-1.

As culturing cells in vitro may alter the function of primary cells, the mesothelial cells may have lost components of the pathways that respond to MSU crystals. It is also possible that the cell culturing process may have selected a population of MSU-crystal-tolerant mesothelial cells while leaving the TLR4 pathway untouched.

Another possibility is that another cell population lining the peritoneal membrane (such as fibroblasts) is predominantly responsible for MCP-1 production upon stimulation, and is mediated by the CD14-dependent TLR4 pathway. This may explain the observation of CD14 blocking the elicited MCP-1 response of the membrane, but not the cultured mesothelial cells.

A follow-up experiment to address these questions could be conducted by excising whole peritoneal tissue, placing pieces with the visceral side of the membrane face down over the top of the culture well already filled with trypsin/EDTA. The lid is then placed over the top in order to provide a seal between the tissue and the well. This experiment would enable the extraction of cells lining the membrane rather than harvesting from the peritoneal cavity as a heterogeneous population. Results should help to confirm whether the cells specifically lining the visceral side of the peritoneum membrane and not those lining the internal organs are responsible for the production of MCP-1 through the CD14dependent TLR4 pathway after stimulation.

Results presented within this section may also warrant a revisit of cytokeratin staining of the peritoneal membrane and identification of MCP-1 producing cells. This would give a 

induced inflammation

further indication of which cell types (other than mesothelial cells) lining the peritoneal membrane are capable in producing MCP-1 upon stimulation.

In summary, these findings have shown that MSU crystals can interact directly with peritoneal membrane cells to induce MCP-1 production, thereby playing a key role in monocyte recruitment during inflammation. The results also indicate that cultured mesothelial cells may be altered in some way or that, although capable of producing MCP1 , they are not predominantly responsible for CD14-sensitive MCP-1 production observed in the whole peritoneal membrane. 


\subsection{The effects of Glatiramer acetate on recruited monocytes in MSU-induced inflammation}

\subsubsection{Monocytes in immunity}

There are many subpopulations of monocytes, however, all are recognized as mononuclear leukocytes of the myeloid lineage. They originate from the bone marrow as myeloid progenitors and are subsequently released into the bloodstream (175). Monocytes either remain in circulation or migrate into peripheral tissues under homeostatic conditions or in response to inflammatory signals (175). A key role of monocytes/macrophages is the phagocytosis and clearance of pathogens and debris, while also contributing to the resolution of inflammation by the production of TGF- $\beta(57,93)$.

\subsubsection{Myeloid-Derived Suppressor Cells}

One phenotype of the recruited inflammatory monocyte/macrophage subset is the myeloidderived suppressor cell (MDSC). MDSCs comprises of a heterogenous population of macrophage precursors, granulocytes, DCs and other myeloid cells at early stages of differentiation $(119,138)$. In murine models of tumours and multiple sclerosis (MS), infiltrating MDSCs have been shown to suppress $\mathrm{CD}^{+} \mathrm{T}$ cell expansion $(137,176,177)$. In addition, recent studies have described circulating MDSCs to be significantly increased in tumour-bearing mice and in cancer patients $(119,176)$. In mice MDSCs are broadly defined as $\mathrm{Gr}-1^{+} \mathrm{CD} 11 \mathrm{~b}^{+}$cells, have been found to accumulate in other models of disease such as sepsis, acute and chronic inflammation, autoimmunity and trauma (137, 176-178). In tumour models especially, different subsets of MDSCs are exploited by the neoplastic tissue to suppress different aspects of the immune system depending on the type of tumour, how the cells were differentiated and the target cell population $(119,132)$.

\subsubsection{Glatiramer acetate associates with blood monocytes}

Multiple sclerosis is an inflammatory autoimmune disease of the CNS, resulting in various neurological deficits such as sensory loss, cognitive defects and paralysis (179). Pathologically, recruited monocytes have been identified as a key cell subset responsible for the production of pro-inflammatory cytokines within the inflamed CNS (180). 
Glatiramer acetate (GA) is an effective treatment of multiple sclerosis and has been shown to suppress experimental autoimmune encephalomyelitis (EAE), the murine model of MS. The drug appears to elicit its effects by altering circulating monocyte functions, which leads to the production of anti-inflammatory cytokines such as IL-10, TFG- $\beta$ or IL-1 receptor antagonist (181-183).

Intravenous administration of Alexa488-GA in mice by members of our group has identified $\mathrm{GA}^{+}$monocytes in the blood expressing $\mathrm{CD} 11 \mathrm{~b}^{+} \mathrm{Gr}-1^{+} \mathrm{F} 4 / 80^{\text {int }} \mathrm{Ly}^{-} \mathrm{C}^{-}$(Appendix 1). Once purified, this circulating population was capable of suppressing $T$ cell proliferation in vitro (Appendix 2). This monocyte phenotype is characteristic of the MDSCs $(119,184)$. However, to distinguish the $\mathrm{GA}^{+}$monocytes from tumour MDSCs, the $\mathrm{CD} 11 \mathrm{~b}^{+} \mathrm{Gr}-1^{\text {hi }}$ cells isolated from the bloodstream in this study will be referred to as suppressor monocytes.

\subsubsection{Objectives}

The aim of this section was to determine if the enhanced $\mathrm{T}$ cell suppressive abilities exhibited by $\mathrm{GA}^{+}$blood suppressor monocytes was retained following recruitment into inflamed tissues. To address this question, we sought to compare the differentiation and $\mathrm{T}$ cell suppressive function between MSU-recruited monocytes from GA-treated and nontreated mice.

\subsubsection{Results}

\subsubsection{T cell suppressive ability of MSU recruited monocytes}

To measure the effect of MSU-recruited monocytes on T cell responses, we chose to set up a $\mathrm{T}$ cell expansion assay based on thymidine uptake $(185,186)$. In this assay, $\mathrm{T}$ cell activation and proliferation is induced by the binding of anti-CD3 and anti-CD28 ligands to their corresponding receptors on the cell surface. This process induces rapid expansion of the $\mathrm{T}$ cell population, which can be quantified by the incorporation of $\left[{ }^{3} \mathrm{H}\right]$-thymidine into the DNA of the dividing cell. The amount of cell proliferation correlates to the levels of radiation that can be measured by a scintillation counter. 
To investigate if the suppressive ability of blood monocytes is retained following recruitment into the peritoneum in MSU-induced inflammation, we first isolated and purified MSU-recruited monocytes at different time-points. C57Bl/6J mice were injected intraperitoneally with $3 \mathrm{mg} / 0.5 \mathrm{~mL}$ MSU crystals. At different time-points, recruited monocytes were isolated from PECs using a Lympholyte ${ }^{\circledR}-\mathrm{M}$ density gradient (described in chapter 3). This purified population comprised between $75 \%$ to $80 \%$ monocytes, as determined by Gr-1 and F4/80 surface marker staining and analysis by flow cytometry.

To obtain a $\mathrm{T}$ cell population for the suppression assay, spleens from naïve C57B1/6J mice were injected with $1-2 \mathrm{~mL}$ of Collagenase II/DNase I solution and incubated for 30 minutes. Following incubation, spleens were disrupted with the back of a syringe plunger and filtered through $40 \mu \mathrm{m}$ cell strainers with cRMPI. Contaminating RBCs were lysed and the remaining splenocytes washed and resuspended in cRMPI. The suppression assay was conducted by co-culture of splenocytes with the purified, recruited monocytes at different cell-to-cell ratios in the presence of $\alpha \mathrm{CD} 3 / \alpha \mathrm{CD} 28$ coated "expander" beads.

As shown in Figure 4.5, early (4 hours) MSU-recruited monocytes were able to completely suppress $T$ cell expansion at 1:1 and 1:2 ratios with decreasing efficacy as the number of splenocytes outnumbered the monocytes (1:4 to 1:16). The recruited monocytes isolated later in the inflammatory response (from 16 hours to 48 hours) displayed an ability to suppress $\mathrm{T}$ cell activation even at 1:8 and 1:16 ratios (Figure 4.5). 
4h

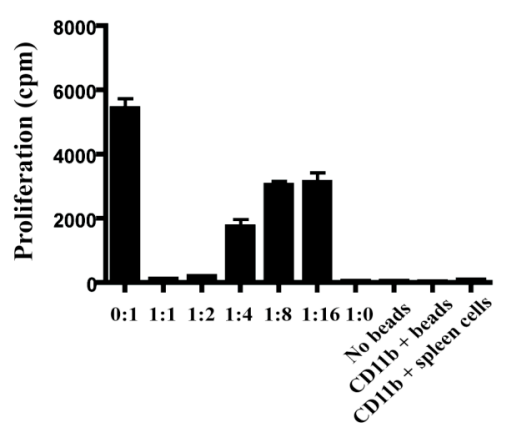

24h

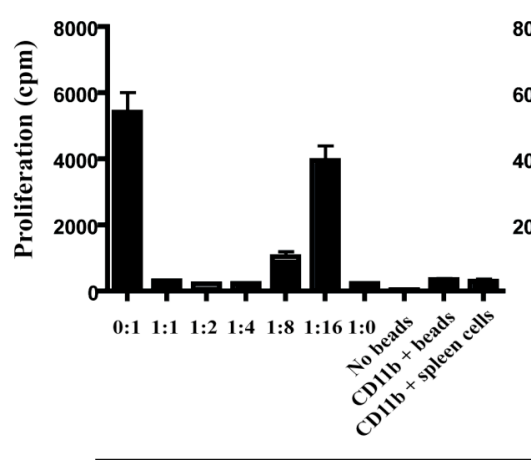

$8 h$

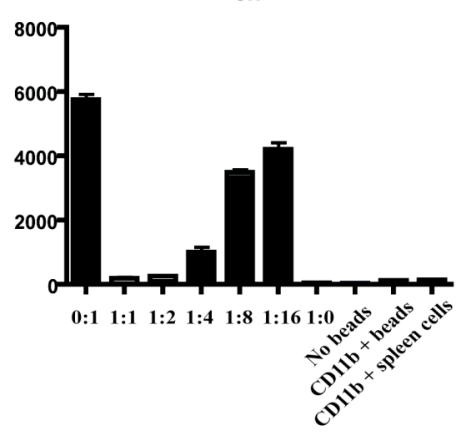

CD11b:Splenocyte ratio

48h
$16 \mathrm{~h}$

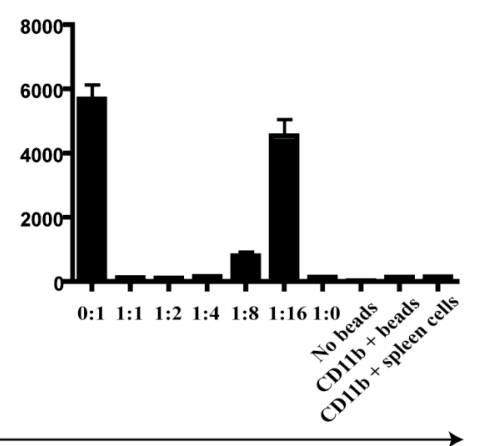

CD11b:Splenocyte ratio
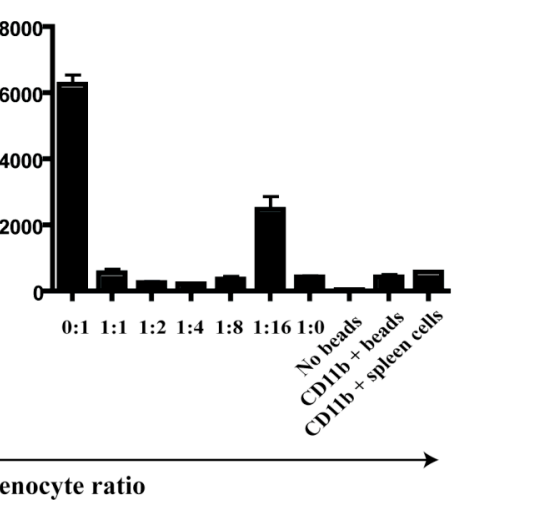

Figure 4.5: Monocyte suppression of splenocyte proliferation induced by DynaBead ${ }^{\circledR}$

expander beads. C57Bl/6J mice were administered with an i.p. injection of MSU crystals $(3 \mathrm{mg} / 0.5 \mathrm{~mL})$. At different time-points, peritoneal exudate cells were harvested and purified by Lympholyte ${ }^{\circledR}-\mathrm{M}$ density gradient $\left(>75 \%\right.$ monocytes). The monocytes were co-cultured with $5 \times 10^{4} \mathrm{C} 57 \mathrm{Bl} / 6 \mathrm{~J}$ splenocytes at the indicated ratios in the presence of DynaBead ${ }^{\circledR} \mathrm{CD} 3 / \mathrm{CD} 28 \mathrm{~T}$ cell expander beads in 96 well round-bottom plates. The cells were incubated for 20 hours, and an additional eight hours in the presence of $\left[{ }^{3} \mathrm{H}\right]$ thymidine. T cell expansion was measured by $\left[{ }^{3} \mathrm{H}\right]$-thymidine incorporation. Measurements performed in triplicate. Values represent mean \pm S.E.M. Results are representative of two independent experiments with four mice per group.

In chapter 3 (section 3.2.9), MSU-recruited monocytes were shown to increase phagocytic function over time. Therefore, it was possible that the observed increase in suppressive activity by monocytes at later time-points was the result of increased uptake of expander beads, thereby decreasing $\mathrm{T}$ cell expansion. To address this issue, a separate proliferation assay was set up where anti-CD3/anti-CD28 antibodies were used to coat culture plates overnight prior to co-incubation of monocytes and $\mathrm{T}$ cells. 
As shown in Figure 4.6, $\mathrm{T}$ cell expansion in response to anti-CD3/anti-CD28 was suppressed by MSU-recruited monocytes isolated from mice 16 hours or more after MSU administration, compared to 4-hour monocytes in the expander bead assays. This result indicated that phagocytosis of expander beads in the previous assay made a significant contribution to the overall level of suppressive activity and particularly for 4- and 8-hour monocytes.

Therefore, in all subsequent suppression assays $\mathrm{T}$ cell expansion was triggered using antiCD3/anti-CD28 coated plates.

$4 h$

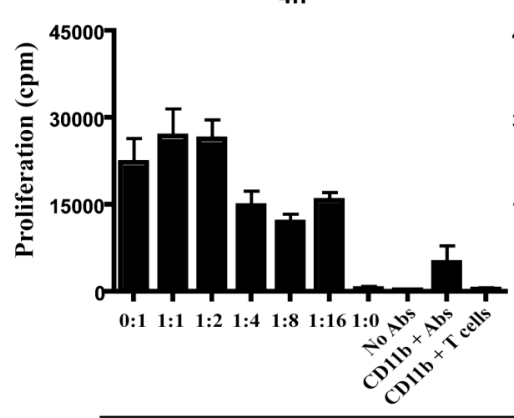

24h

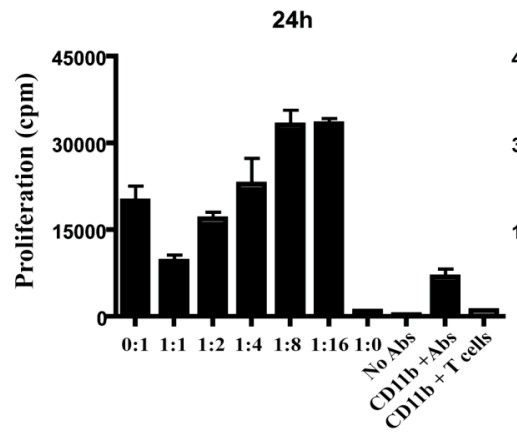

$8 h$

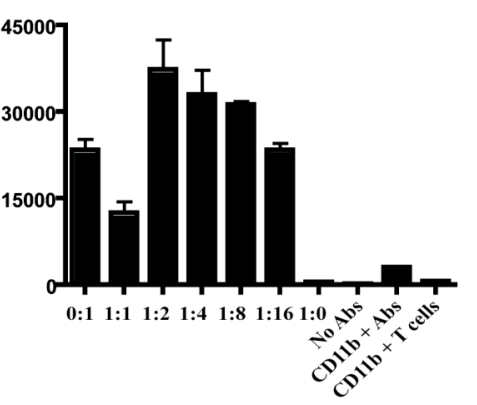

CD11b:T cell ratio

$48 h$

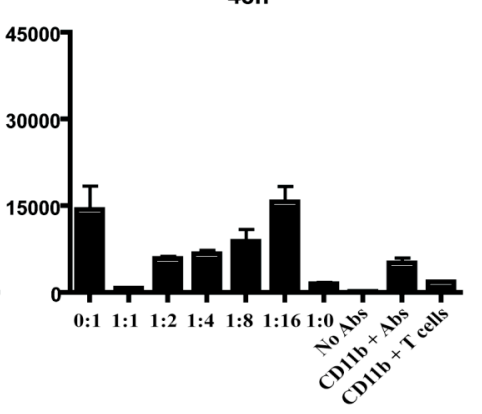

$96 \mathrm{~h}$

$16 h$
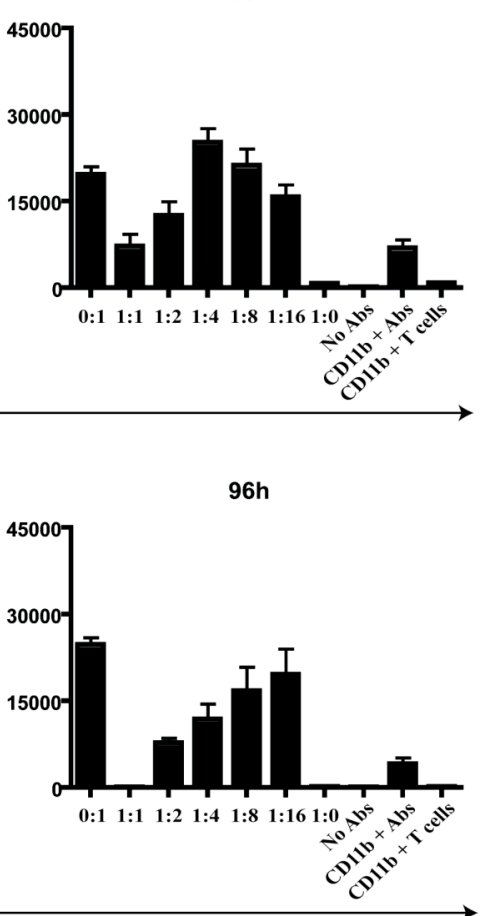

CD11b:T cell ratio

Figure 4.6: Monocyte suppression of polyclonal $T$ cell proliferation induced by anti-

mouse CD3/CD28 coated plates. C57B1/6J mice were administered with an i.p. injection of MSU crystals $(3 \mathrm{mg} / 0.5 \mathrm{~mL})$. At different time-points, peritoneal exudate cells were harvested and purified by Lympholyte ${ }^{\circledR}-\mathrm{M}$ density gradient ( $>75 \%$ monocytes). The monocytes were co-cultured with $5 \times 10^{4} \mathrm{C} 57 \mathrm{Bl} / 6 \mathrm{~J}$ lymphocytes at the indicated ratios in 96 well round-bottom plates pre-coated with anti-mouse CD3 and CD28 antibodies $(2 \mu \mathrm{g} / \mathrm{mL}$ and $3 \mu \mathrm{g} / \mathrm{mL}$ respectively) in D-PBS the night prior. The cells were incubated for 20 hours, and an additional eight hours in the presence of $\left[{ }^{3} \mathrm{H}\right]$-thymidine. T cell expansion was measured by $\left[{ }^{3} \mathrm{H}\right]$-thymidine incorporation. Measurements performed in triplicate. Values represent mean \pm S.E.M. Results are representative of three independent experiments with four mice per group. 
In addition, to improve the purity of the $\mathrm{T}$ cell population, all subsequent suppression assays were carried out using $\mathrm{T}$ cell preparations from naïve inguinal lymph nodes. To obtain the $\mathrm{T}$ cell population, inguinal lymph nodes from naïve $\mathrm{C} 57 \mathrm{Bl} / 6 \mathrm{~J}$ mice were disrupted with the back of a syringe plunger and filtered through $40 \mu \mathrm{m}$ cell strainers with cRMPI. Contaminating RBCs were lysed and the lymphocytes washed and resuspended in cRMPI.

As the suppressive ability of recruited monocytes appeared to increase as they differentiated to more macrophage-like cells, we next sought to investigate whether resident macrophages displayed an innate ability to suppress $\mathrm{T}$ cell proliferation. PECs were harvested from naïve $\mathrm{C} 57 \mathrm{BBl} / 6 \mathrm{~J}$ mice, however they were not put through a density gradient as resident macrophages (approximately $50-70 \mu \mathrm{m}$ in diameter), due to their relatively large size, cannot be separated by this particular method. As the peritoneal lavage normally contains around $50 \%$ or more macrophages, the exudate cells were washed and co-cultured with $\mathrm{T}$ cells from naïve mice in anti-CD3/anti-CD28 pre-coated culture plates. After the overnight incubation, T cell proliferation was measured by $\left[{ }^{3} \mathrm{H}\right]-$ thymidine incorporation. The results showed that resident macrophages displayed an ability to suppress $\mathrm{T}$ cell proliferation when macrophage and $\mathrm{T}$ cell numbers are approximately equal (Figure 4.7). However, the suppressive ability was lost when $\mathrm{T}$ cell numbers far outnumbered the macrophage population (Figure 4.7). 


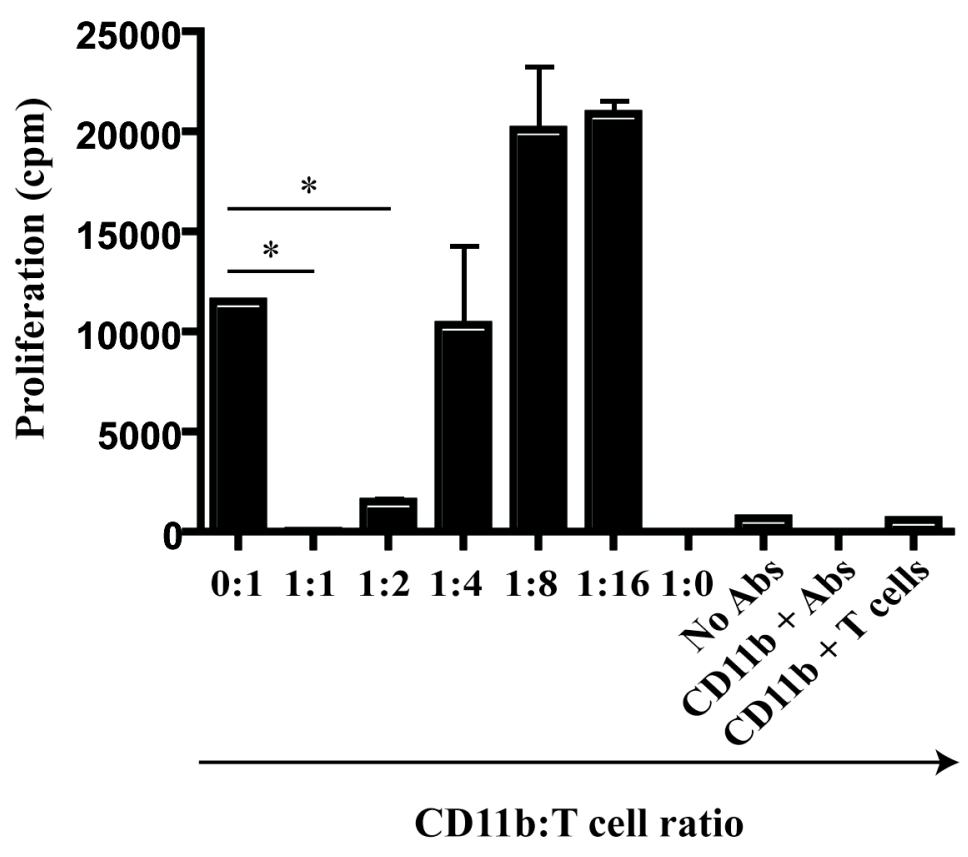

Figure 4.7: Resident macrophage suppression of polyclonal $T$ cell proliferation induced by anti-mouse CD3/CD28 coated plates. Peritoneal exudate cells were harvested from naïve C57B1/6J mice. The cell suspension (>50\% macrophages) was then co-cultured with $5 \times 10^{4} \mathrm{C} 57 \mathrm{Bl} / 6 \mathrm{~J}$ lymphocytes at the indicated ratios in 96 well round-bottom plates pre-coated with anti-mouse CD3 and CD28 antibodies $(2 \mu \mathrm{g} / \mathrm{mL}$ and $3 \mu \mathrm{g} / \mathrm{mL}$ respectively) in D-PBS the night prior. The cells were incubated for 20 hours, and an additional eight hours in the presence of $\left[{ }^{3} \mathrm{H}\right]$-thymidine. T cell expansion was measured by $\left[{ }^{3} \mathrm{H}\right]$-thymidine incorporation. Measurements performed in duplicate. Values represent mean \pm S.E.M. $*=P$ $<0.05$ as determined by one-way ANOVA and Tukey multiple comparison test. Results are representative of two independent experiments with five mice per group.

In summary, these results showed that infiltrating monocytes do not retain their $\mathrm{T}$ cell suppressive abilities immediately after recruitment. However, at later time-points along the inflammatory response they develop the ability to suppress $T$ cell expansion. This indicated that as monocytes differentiate into more macrophage-like cells, they are able to non-specifically suppress $\mathrm{T}$ cell proliferation.

\subsubsection{GA labelling of circulating monocytes}

We next set out to determine whether MSU-recruited $\mathrm{GA}^{+}$monocytes could enhance T cell suppressive activity compared to normal MSU-recruited monocytes. 

induced inflammation

Before investigating the effects of $\mathrm{GA}^{+}$monocytes in the MSU-induced inflammatory model, we first confirmed the phenomenon of in vivo labelling of circulating monocytes by GA. C57Bl/6J mice were injected i.v. with GA-Alexa488. After thirty minutes, white blood cells were isolated from blood, stained with CD11b FACS antibody and analysed by flow cytometry (Figure 4.8).

The $\mathrm{GA}^{+}$cells in the blood were confirmed as expressing high levels of CD11b $\left(\mathrm{CD} 11 \mathrm{~b}^{+}\right)$ and were located to the mononuclear fraction on the side scatter (SSC ${ }^{\text {low }}$ ). Studies by other members of our group looking at other cell surface antigens further classified these cells as Ly6G, Gr- $1^{+}$and $\mathrm{F} 4 / 80^{\text {int }}$ (Appendix 1), thus confirming $\mathrm{GA}^{+}$cells as circulating inflammatory monocytes rather than neutrophils

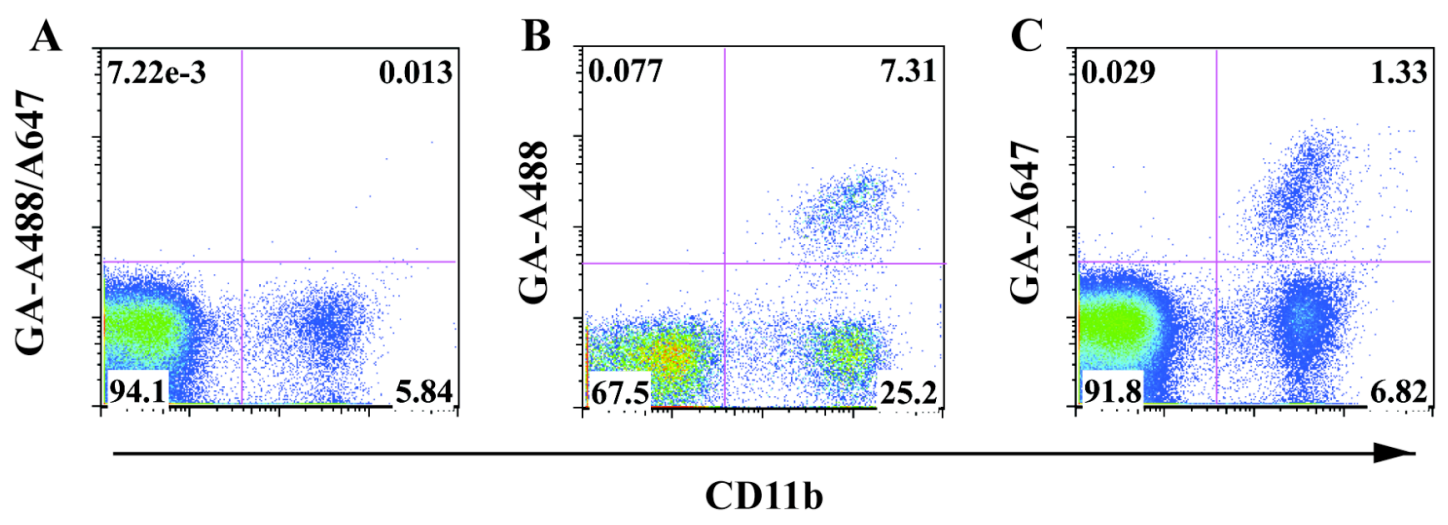

Figure 4.8: GA binds $\mathrm{CD} 11 \mathrm{~b}^{+}$cell population after i.v. injection. C57B1/6J mice were either A. left untreated or administered with an i.v. injection of B. Alexa488- or C. Alexa647-labelled GA $(7 \mu \mathrm{g} / \mathrm{mL})$ in D-PBS. Cells were recovered 30 minutes after the injections, stained for cell surface antigens and samples of individual mice analysed by flow cytometry. Results are representative of at least three independent experiments.

To determine how long $\mathrm{GA}^{+}$monocytes remained in circulation, Alexa488-GA was injected intravenously, and blood cells of individual mice collected at different time-points and analysed by flow cytometry.

These results demonstrated that administration of GA-Alexa488 i.v. resulted in the specific labelling of $90-100 \%$ of circulating monocytes and that the $\mathrm{GA}^{+}$cells remained present in the bloodstream for up to 18 hours after injection. However, the mean fluorescence index 
(MFI) of GA bound to monocytes began to decline after an hour. At 48 hours, the number of $\mathrm{GA}^{+}$monocytes was close to undetectable and even then the fluorescence intensity from the dye was relatively low.

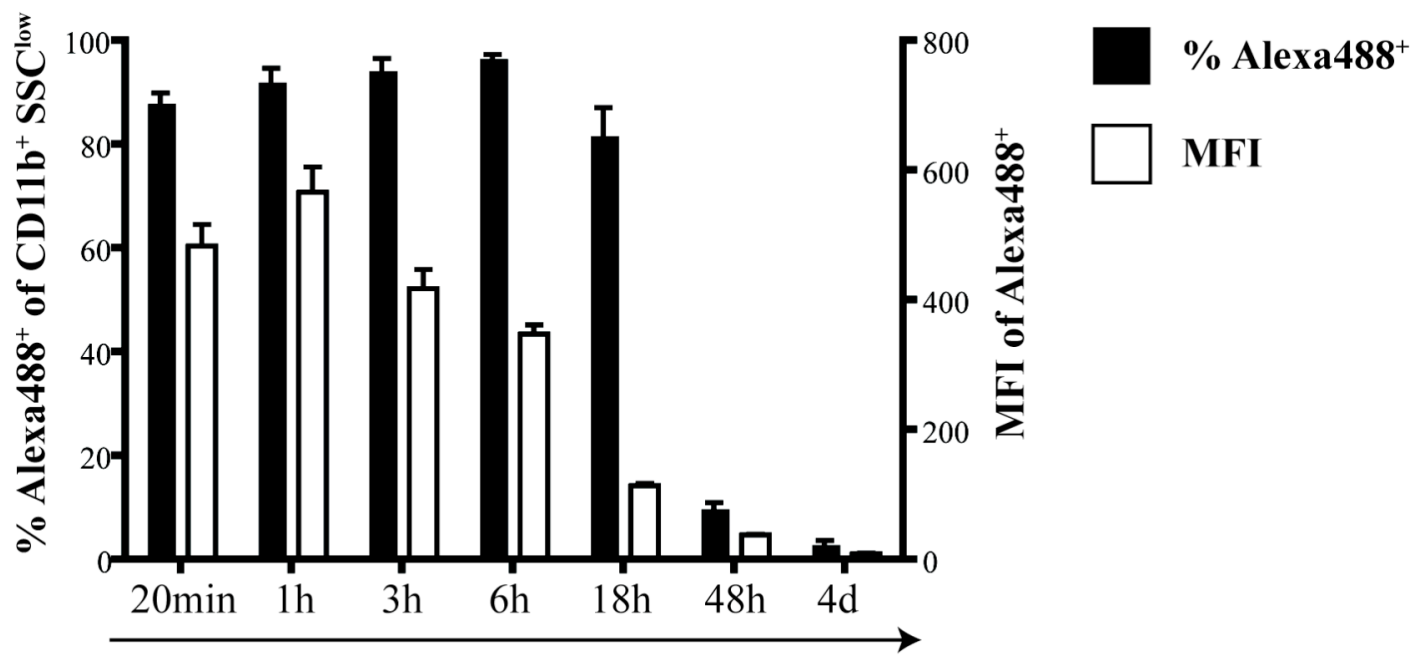

Timepoints

Figure 4.9: GA binding of blood monocytes is stable over 18 hours. C57B1/6J mice were administered with or without an i.v. injection of GA-Alexa488 $(7 \mu \mathrm{g} / \mathrm{mL})$ in D-PBS. After the injections, mice were tail-bled at various time-points and the cells stained for cell surface antigens and samples of individual mice analysed by flow cytometry. Values represent mean \pm S.E.M. Time-courses were split into two separate experiments, for time ranges of 20 minutes to six hours and from six hours to 96 hours. Results are representative of four independent experiments (both time-courses each performed twice) with three mice per group.

To take full advantage of the period when $\mathrm{GA}^{+}$cells remained in the bloodstream, GA i.v. injections were immediately followed by i.p. MSU administration. This ensured that circulating $\mathrm{GA}^{+}$cells in circulation were available for MSU-induced recruitment.

\subsubsection{3 $\mathrm{GA}^{+}$monocytes can be recruited into the peritoneum in MSU- induced inflammation.}

To determine whether the $\mathrm{GA}^{+}$monocytes could be recruited into the peritoneum by MSUinduced inflammation, GA-A488 was injected intravenously into $\mathrm{C} 57 \mathrm{Bl} / 6 \mathrm{~J}$ mice, followed immediately by an intraperitoneal injection of MSU crystals. After 6 hours, cells were harvested and stained for CD11b, F4/80 and Gr-1 and analysed by flow cytometry. FACS plots showed that GA-untreated mice did not stain positive for Alexa488 (Figure 4.10A, 
gate R1) compared to GA-treated mice. GA-treated mice exhibited a distinct population of $\mathrm{CD}_{11 \mathrm{~b}^{+} \text {GA-Alexa488 }}^{+}$double positive cells (Figure 4.10B, gate R1). The CD11b $\mathrm{GA}^{+}$ population was confirmed as recruited monocytes by their intermediate expression of the macrophage marker F4/80 and showed both a Gr- ${ }^{\text {hi }}$ and $\mathrm{Gr}-1^{\text {int }}$ population (Figure $4.10 \mathrm{C}$, gate R1). This is consistent with the presence of both $\left(\mathrm{Gr}-1^{+}\right)$newly recruited monocytes and recruited monocytes that have downregulated Gr-1 as they differentiate into macrophages observed in chapter 3, section 3.2.7.

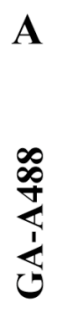

A

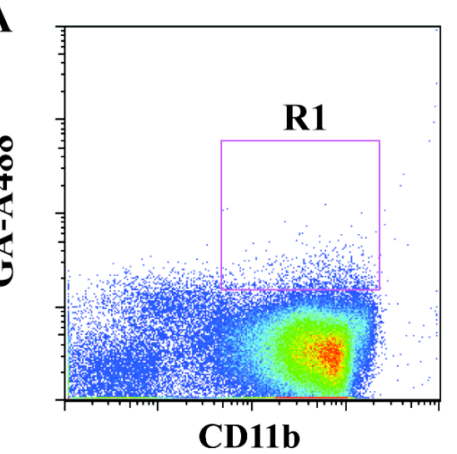

B

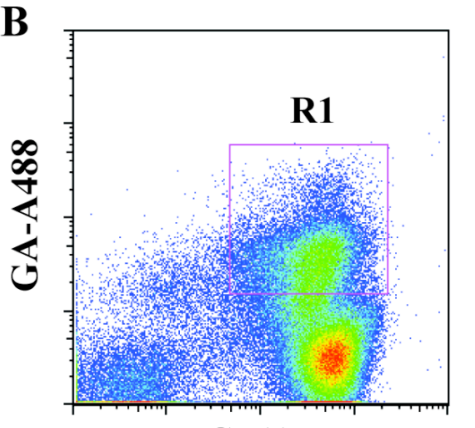

CD11b

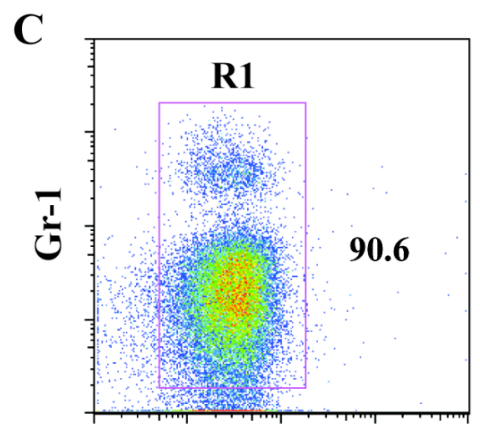

F4/80

Figure 4.10: GA bound cells are recruited into the peritoneum after MSU induced

inflammation. C57B1/6J mice were administered with or without an i.v. injection of GA-A488 $(7 \mu \mathrm{g} / \mathrm{mL})$ in D-PBS. After 20 minutes, mice were administered with MSU crystals $(3 \mathrm{mg} / 0.5 \mathrm{~mL})$. Peritoneal exudate cells were then harvested after six hours, stained for CD11b, F4/80 and Gr-1 cell surface antigens and analysed by flow cytometry. A. Peritoneal exudate cells from GA untreated mice were used as negative controls (gate R1). B. Peritoneal exudate cells from GA-A488 labelled mice were identified as CD1 $1 \mathrm{~b}^{+}$and GA-A $488^{+}$(gate R1). C. Frequency of GA-A488 ${ }^{+}$cells (gate R1) showing monocyte surface marker phenotypes, $\mathrm{Gr}-1^{\text {int }}$ and $\mathrm{F} 4 / 80^{\text {low }}$. Results are representative of at least three independent experiments.

\subsubsection{Effects of GA treatment on $\mathrm{T}$ cell suppressive ability of MSU recruited monocytes}

The results above confirmed that MSU-induced inflammation was able to recruit $\mathrm{GA}^{+}$ monocytes from the blood stream into the peritoneum. We next asked the question; do $\mathrm{GA}^{+}$monocytes recruited from the blood exhibit enhanced T cell suppressive activity?

Mice were given GA i.v. immediately prior to MSU administration i.p and PECs were harvested at 6 and 18 hours to align with period of maximum staining of GA-monocytes in the bloodstream (Figure 4.9). The MSU-recruited monocytes were purified using the 

induced inflammation

lympholyte ${ }^{\circledR}-\mathrm{M}$ density gradient and tested for their ability to suppress CD3/CD28 induced $\mathrm{T}$ cell expansion. As previously described in Figure 4.6, non-GA-treated recruited monocytes exhibited suppressive activity at 18 hours but not at 6 hours (Figure 4.11A). Interestingly, GA-treatment appeared to exacerbate $\mathrm{T}$ cell expansion at both time-points (Figure 4.11B

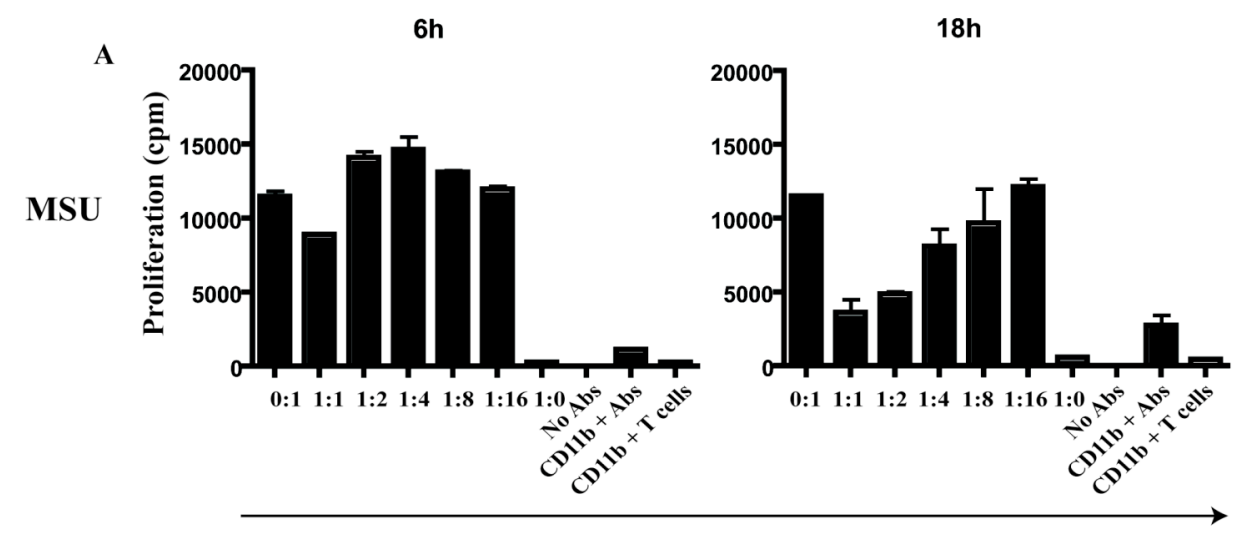

CD11b: T cell ratio

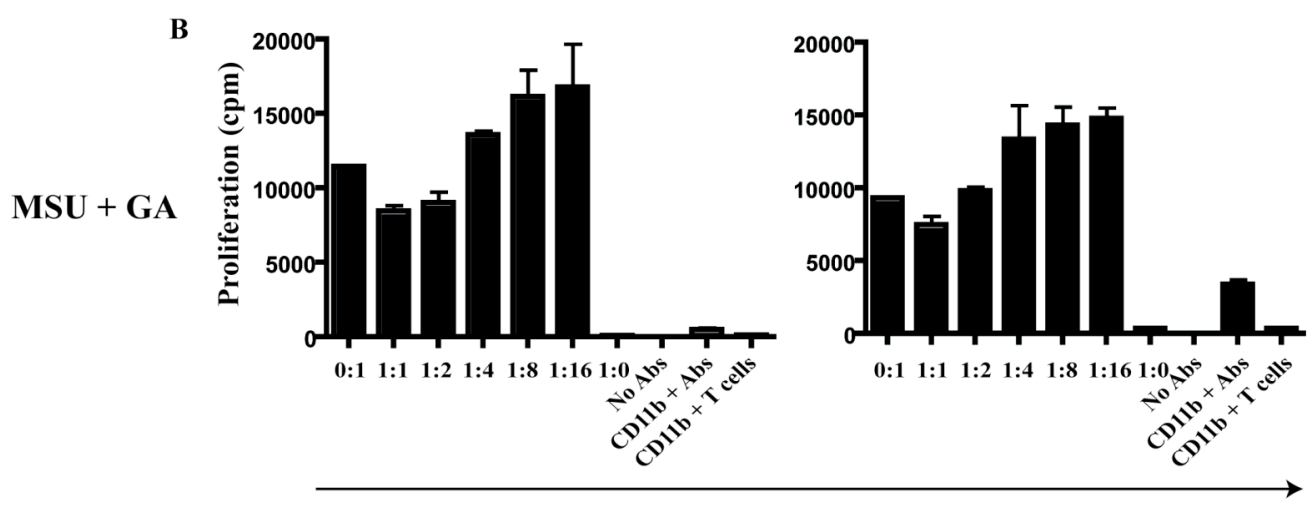

CD11b:T cell ratio

Figure 4.11: GA bound monocytes does not alter their suppressive abilities on polyclonal $\mathbf{T}$ cell proliferation. $\mathrm{C} 57 \mathrm{~B} 1 / 6 \mathrm{~J}$ mice were administered with or without an i.v. injection of GA-A488 $(7 \mu \mathrm{g} / \mathrm{mL})$ in D-PBS. After 20 minutes, mice were administered with MSU crystals $(3 \mathrm{mg} / 0.5 \mathrm{~mL})$ for six and 18 hours. Peritoneal exudate cells were harvested and purified by Lympholyte ${ }^{\circledR}-\mathrm{M}$ density gradient ( $>75 \%$ monocytes). The monocytes were co-cultured with $5 \times 10^{4}$ C57B1/6J lymphocytes at the indicated ratios in 96 well round-bottom plates pre-coated with anti-mouse CD3 and CD28 antibodies $(2 \mu \mathrm{g} / \mathrm{mL}$ and $3 \mu \mathrm{g} / \mathrm{mL}$ respectively) in D-PBS the night prior. The cells were incubated for 20 hours, and an additional eight hours in the presence of $\left[{ }^{3} \mathrm{H}\right]$-thymidine. T cell expansion was measured by $\left[{ }^{3} \mathrm{H}\right]$-thymidine incorporation. Measurements performed in triplicate. Values represent mean \pm S.E.M. Results are representative of two independent experiments with four mice per group. 

induced inflammation

\subsubsection{Effects of GA treatment on MSU recruited monocyte phenotype}

Next we sought to determine if GA altered other phenotypic features on MSU-recruited monocytes. To do this, we compared the differentiation markers of MSU-recruited monocytes from GA-treated to untreated mice. As in the previous experiments, PECs were harvested and stained with cell surface markers CD11b, F4/80 and Gr-1 and analysed by flow cytometry. GA bound monocytes displayed no differences in the main differentiation cell surface markers compared to non-GA-treated monocytes (Figure 4.12).

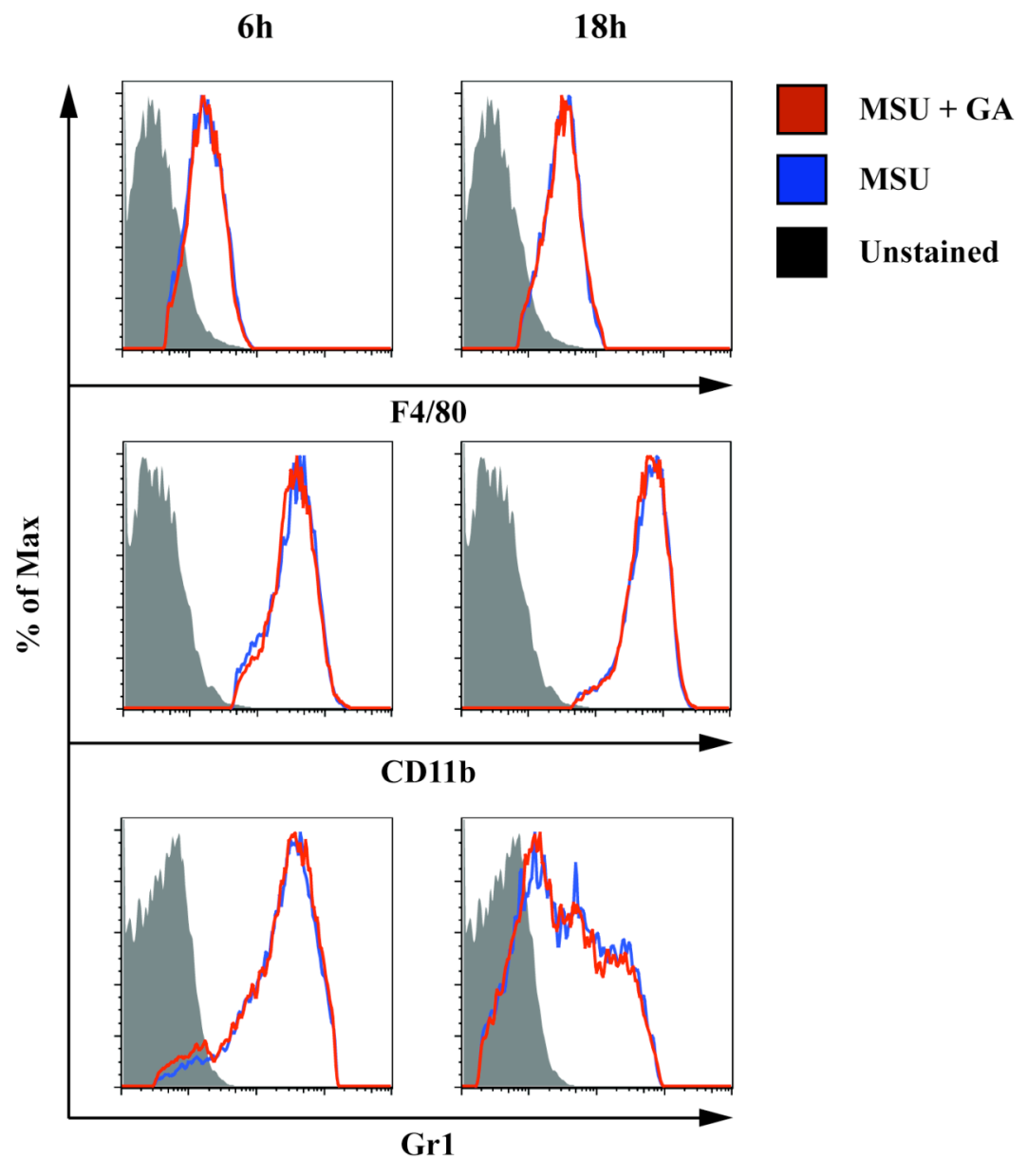

Figure 4.12: GA bound monocytes do not alter their surface marker differentiation profile. C57Bl/6J mice were administered with or without an i.v. injection of GA-A488 $(7 \mu \mathrm{g} / \mathrm{mL})$ in DPBS. After 20 minutes, mice were administered with MSU crystals $(3 \mathrm{mg} / 0.5 \mathrm{~mL})$ for six and 18 hours. Peritoneal exudate cells were harvested and stained for cell surface antigens and samples of individual mice analysed by flow cytometry. Plots are gated on $\mathrm{Gr}^{\text {in }}$ and $\mathrm{F} 4 / 80^{\text {int }}$ monocytes. Results are representative of three independent experiments. 


\subsubsection{Effects of GA treatment on MSU recruited monocyte phagocytic function}

To determine whether phagocytic function of MSU-recruited monocytes was altered after GA treatment, PECs were recovered 6 and 18 hours after MSU administration and recruited monocytes purified by density gradient separation. Monocytes were then incubated with fluorescent beads, stained for the cell surface antigen F4/80 (to identify the bead $^{+}$monocyte population) and analysed by flow cytometry.

As shown in Figure 4.14, the percentages of bead ${ }^{+}$monocytes were similar in both GAtreated and non-treated groups. This indicated that GA treatment did not alter the phagocytic ability of recruited monocytes.
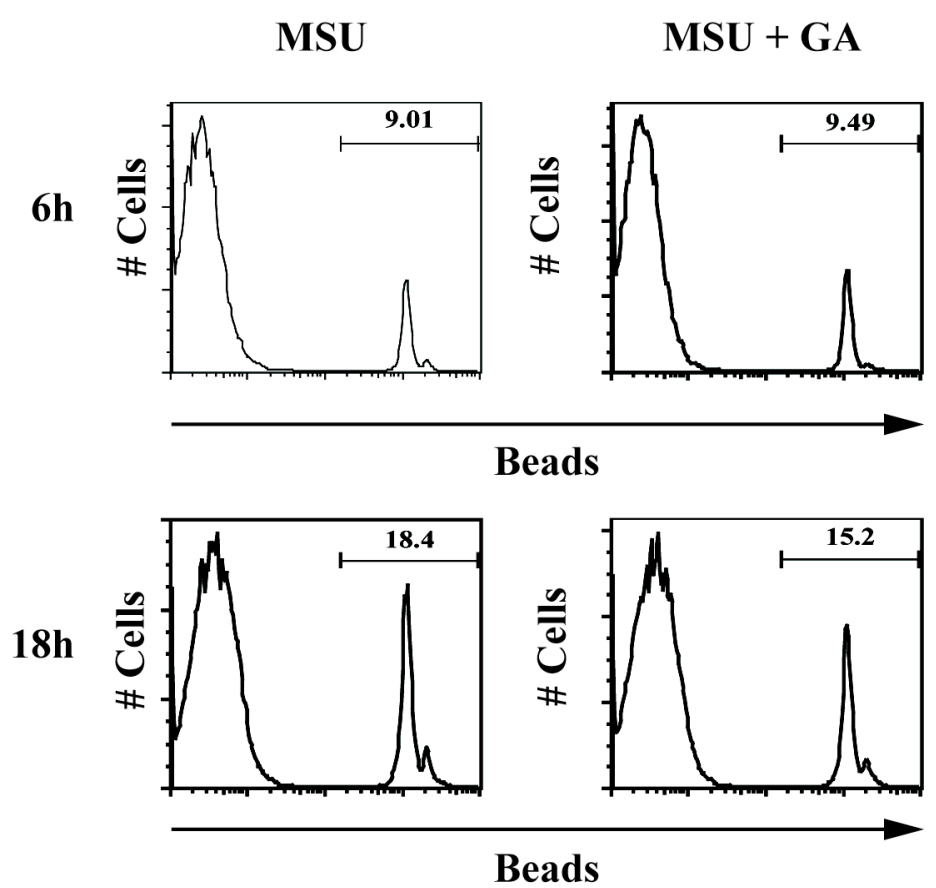

Figure 4.13: GA treatment does not alter phagocytic ability of MSU-recruited monocytes. $\mathrm{C} 57 \mathrm{~B} 1 / 6 \mathrm{~J}$ mice were administered with or without an i.v. injection of GA-A $488(7 \mu \mathrm{g} / \mathrm{mL})$ in D-PBS. After 20 minutes, mice were administered with MSU crystals $(3 \mathrm{mg} / 0.5 \mathrm{~mL})$ for six and 18 hours. Peritoneal exudate cells were harvested and purified by Lympholyte ${ }^{\circledR}-\mathrm{M}$ density gradient ( $>75 \%$ monocytes). Cells were resuspended at $5 \times 10^{5}$ cells $/ \mathrm{mL}$ and $2 \mathrm{~mL}$ of the suspension was incubated with fluorescent beads for 30 minutes. Excess beads were washed off and cells were stained for the cell surface antigen F4/80 and samples of individual mice analysed by flow cytometry. Plots are gated on F4/80 int monocytes. Results are representative of one experiment with three mice per group. 
In the same experiment, the capacity of monocytes to take up more than one bead was also evaluated (chapter 3, section 3.2.9). This involved gating on the bead-positive population and then sub-gating on the populations displaying a second fluorescent spike. The ability of MSU-recruited $\mathrm{GA}^{+}$monocytes to phagocytose more than one bead was comparable to normal recruited monocytes (Figure 4.14).

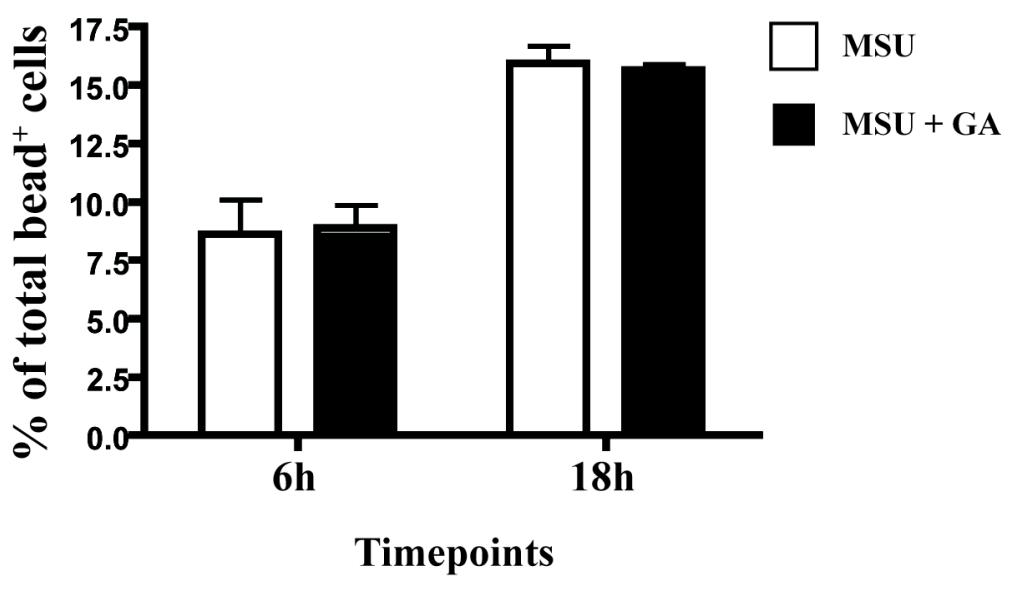

Figure 4.14: GA treatment does not alter phagocytic capacity of MSU-recruited monocytes. Total $\mathrm{F} 4 / 80^{+}$and $\mathrm{Bead}^{+}$cells in Figure 4.11 were analysed for their ability to phagocytose more than one bead using histogram peaks in flow cytometry. Measurements were performed in duplicate. Values represent mean \pm S.E.M. Results are representative of one experiment with three mice per group.

\subsubsection{Effects of GA treatment on recruited monocyte functionality}

We next looked at the effect of GA on the functional response by restimulating recruited monocytes with MSU crystals or LPS. GA-A488 was injected intravenously into naïve C57B1/6J mice followed by an intraperitoneal injection of MSU crystals. At 6 and 18 hours, PECs were harvested and purified through a density gradient. Monocytes were then cultured in the presence of LPS or MSU crystals for 8 hours, and the supernatant analysed for cytokine/chemokines levels by ELISA or Bioplex bead array.

There were no significant increases in the production of IL-6, MCP-1 or KC after MSU crystal or LPS restimulation by MSU-recruited monocytes from GA-treated compared to non-GA-treated mice (Figure 4.15). 

induced inflammation

A

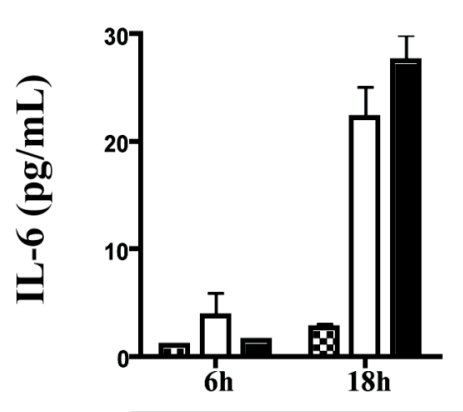

\section{LPS}

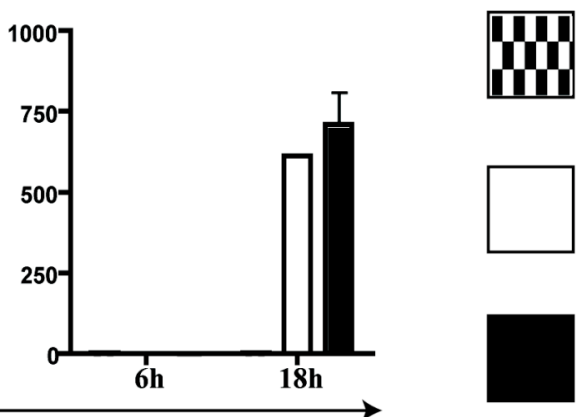

Unstimulated

MSU

MSU + GA

B
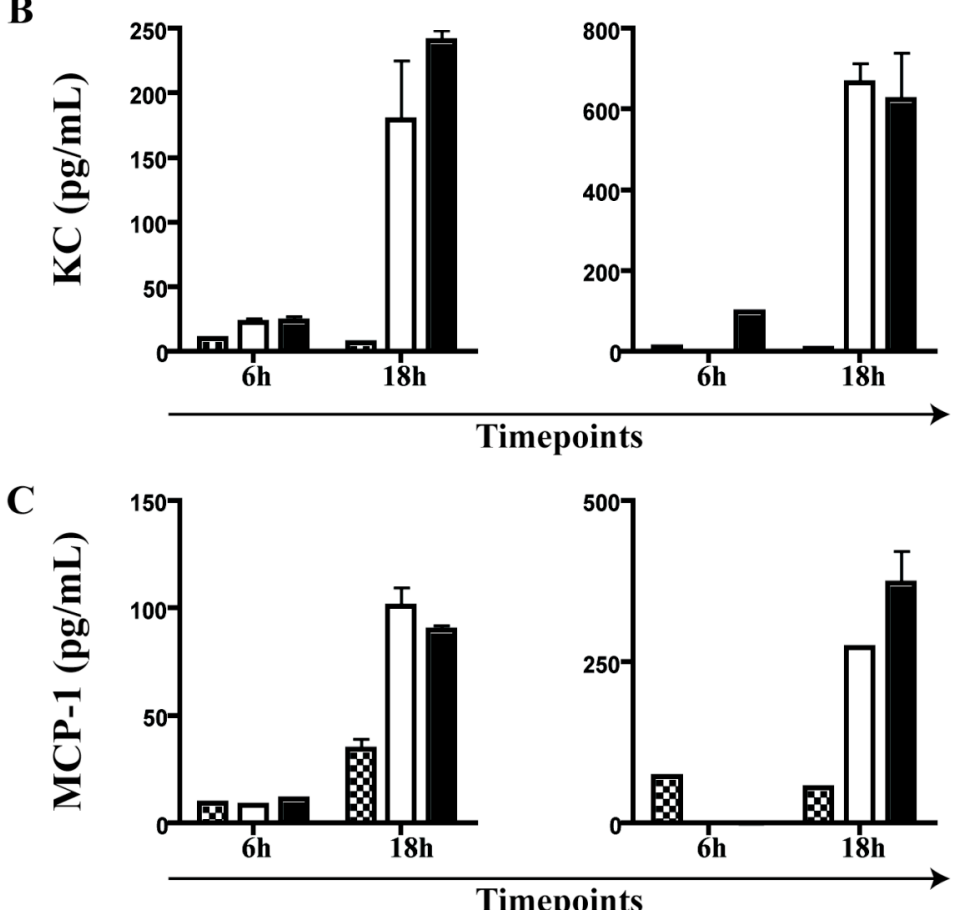

Timepoints

Figure 4.15: GA bound monocytes do not alter their capacity to produce cytokines/chemokines after restimulation. C57Bl/6J mice were administered with or without an i.v. injection of GA-A488 $(7 \mu \mathrm{g} / \mathrm{mL})$ in D-PBS. After 20 minutes, mice were administered with MSU crystals $(3 \mathrm{mg} / 0.5 \mathrm{~mL})$ for six and 18 hours. Peritoneal exudate cells were harvested and purified by Lympholyte ${ }^{\circledR}-\mathrm{M}$ density gradient ( $>75 \%$ monocytes). Cells were resuspended at $5 \times 10^{5}$ cells $/ \mathrm{mL}$ and $200 \mu \mathrm{L}$ was cultured in the presence of MSU $(500 \mu \mathrm{g} / \mathrm{mL})$ or LPS $(100 \mathrm{ng} / \mathrm{mL})$ for eight hours. After the incubation period, the levels of A. IL-6 B. KC C. MCP-1 were determined by ELISA and Bioplex bead array. Measurements performed in duplicate. Values represent mean \pm S.E.M. Results are representative of one experiment with three mice per group. 


\subsubsection{Discussion}

Purified naïve blood monocytes have been shown previously by other members of our group to display an intrinsic ability to suppress expanding polyclonal $\mathrm{T}$ cells in vitro. While MDSCs also display monocyte-like phenotypes $\left(\mathrm{Gr}-1^{+} \mathrm{CD} 11 \mathrm{~b}^{+}\right)$and have the ability to suppress $\mathrm{T}$ cell proliferation, it is a common perception that these are induced by nonhost derived factors, such as tumours $(138,187,188)$.

In this study, the importance of choosing the correct in vitro method of activation was made clear during the optimisation of the suppression assay. When purified MSUrecruited monocytes were co-cultured with $\mathrm{T}$ cells in the presence of expander beads, the development of monocyte suppressive ability at the different time-points were almost methodical. In fact, the results were artificial effects of increases in monocyte phagocytic function, correlating with the differentiation into more macrophage-like cells. The uptake of the expander beads by monocytes blocked $\mathrm{T}$ cell expansion giving a false and exaggerated profile of $\mathrm{T}$ cell suppressive activity. These observations serve as a technical caveat, emphasizing that $\alpha \mathrm{CD} 3 / \alpha \mathrm{CD} 28$ expander beads can be used in $\mathrm{T}$ cell expansion assays with non-phagocytic cells, however when working with phagocytes such as monocytes/macrophages, the use of antibody coated plates avoids the possibility of false positive results.

During the early stages of MSU-induced inflammation, monocytes migrate into a chaotic environment filled with infiltrated neutrophils $(189,190)$, debris and a mixture of proinflammatory cytokines/chemokines $(40,41,43)$ and growth factors (151). Unlike their naïve counterparts in blood, early recruited monocytes do not suppress $\mathrm{T}$ cell activation. This may be due to the specific inflammatory environment into which monocytes are recruited; for example, the tumour environment (IL-17 and TNF $\alpha$ ) $(191,192)$ may support or drive the monocyte suppressive phenotype often associated with immune tolerance $(119,176,188)$. In the acute MSU-induced inflammatory environment consisting of predominately IL-6, IL-1 $\beta$, TNF $\alpha(40,43)$ and soluble factors secreted by neutrophils (such as IL-8); these pro-inflammatory signals could override the recruited-monocyte T cell suppressive functions. 
Over time MSU-recruited monocytes did regain their suppressive phenotype; this appeared to correlate with the loss of hemopoietic markers (such as 7/4 and Gr-1) and increase in F4/80 expression as they differentiated into macrophages (chapter 3, section 3.2.7). While the changes in monocyte surface markers indicate gradual development of more macrophage-like characteristics, it appears that the acute pro-inflammatory environment can induce a state of inactivity from the monocytes until after the acute inflammatory response resolves. This may correlate with the uptake of neutrophils by recruited monocytes in order to initiate the resolution of the inflammatory response (described in chapter 3). By holding back the development of the suppressive phenotype, the inflammatory response is allowed to run its course before "semi-differentiated" monocytes can continue with progression in functionality.

One of the key findings of this study was that MSU-recruited $\mathrm{GA}^{+}$monocytes did not display any $\mathrm{T}$ cell suppressive ability but instead exacerbated $\mathrm{T}$ cell expansion compared to monocytes from non-GA-treated mice. The monocyte populations from GA-treated and non-treated mice did not differ in their differentiation profiles, phagocytic functions or capacity to produce IL-6, MCP-1 or KC. In Chapter 3, I demonstrated that monocytes recruited into an MSU-induced acute inflammatory environment are programmed to develop macrophage-like phenotypes and pro-inflammatory functions. This microenvironment could, in the process of driving monocyte to macrophage differentiation, alter GA's modulatory effects on its target cell type. It is possible that the modulatory effects of GA on recruited monocytes is specific to the EAE microenvironment (IFN- $\gamma$ and IL-17), which promotes the differentiation of $\mathrm{GA}^{+}$monocytes to a phenotype that can suppress $\mathrm{T}$ cell expansion. Further studies to investigate whether the inflammatory environment plays a key role in GA's modulatory effects on recruited monocytes could involve investigating the activity of arginase-1, which has been indicated as a mechanism of suppression by MDSCs in EAE $(193,194)$, and comparing its activity between $\mathrm{GA}^{+}$monocytes in an MSU-induced acute inflammatory and the EAE autoimmune environment. 

induced inflammation

Another possible explanation for the loss of function may be a change in GA's modulatory effects on monocytes as they transmigrate through the endothelium. It has been previously shown that interactions with endothelial cells can cause the altered expression of several genes (such as MMP-1, MCP-1 and TG2) on human monocytes (114). A similar modification may have occurred with the $\mathrm{GA}^{+}$monocytes after recruitment into the peritoneum resulting in the loss of their abilities to suppress $\mathrm{T}$ cell activation.

In summary, the results presented in this section have shown that the $\mathrm{T}$ cell suppressive activities of MSU-recruited monocytes develop as they differentiate into macrophages. In addition, $\mathrm{GA}^{+}$monocytes do not suppress and in fact exacerbate $\mathrm{T}$ cell expansion in an MSU-induced inflammatory environment. 
Chapter 5.0:

General Discussion 
There have been many studies in different disease models exploring the differentiation and functional contributions of recruited monocytes to pathology. The objectives of this study offer insight into the importance of an acute inflammatory environment on the differentiation and function of recruited monocytes. The profiling of monocytes recruited into a microenvironment devoid of resident macrophages and infiltrating neutrophils provided a means to study monocyte differentiation and function in an attenuated inflammatory environment.

\subsection{Initiation of MSU-induced leukocyte recruitment}

In the murine model of acute gout, MSU administration elicits activation of resident macrophages and induces the recruitment of circulating neutrophils and monocytes into the peritoneal cavity.

Infiltrating neutrophils are important potentiators of acute inflammation through the production of IL-8 (87) and superoxide (46). The recruitment of neutrophils from the bloodstream was previously believed to be mediated by the production of TNF $\alpha$ by macrophages after LPS administration in the air pouch model (195). However, other studies have shown that when resident macrophages are depleted prior to MSU crystal stimulation i.p., abrogation of neutrophil recruitment correlated with a similar decrease in the chemokine KC while TNF $\alpha$ levels were unaffected (58). The results presented within this study are consistent with the latter finding, showing that depletion of resident macrophages in vivo results in abrogated neutrophil recruitment and decreased $\mathrm{KC}$ production within the peritoneum during MSU-induced inflammation (chapter 3, section 3.2.3). This indicates that resident macrophages play a prominent role in neutrophil recruitment through the production of $\mathrm{KC}$ in the murine peritoneal model of MSU-induced acute inflammation.

The mechanisms responsible for MSU-induced monocyte recruitment into inflamed tissues are less well understood. In this study, activation of resident macrophages after MSU administration was observed in conjunction with increased levels of the monocyte 
chemokine MCP-1 in vivo (chapter 3, section 3.2.4). However, the source of MCP-1 production was unclear.

Data reported by Olivier and colleagues indicate that murine macrophage cell-lines exposed to MSU crystals transiently increase mRNA levels of various chemokines (including MCP-1) (69). Neutropenic mice are also reported to be unable to mobilize inflammatory monocytes from the circulation after LPS stimulation in a subcutaneous air pouch model (165). Consistent with this, rabbits made neutropenic following antibody administration are also unable to retain recruited monocytes in the alveolar space in a model of pulmonary inflammation (169). Together these data would support a potential role for both resident macrophages and infiltrating neutrophils in monocyte recruitment in acute MSU-induced inflammation.

However, other in vivo findings have shown that activated macrophages do not produce MCP-1 after MSU-stimulation (58) and that in LFA-1 ${ }^{-/}$mice, neither MCP-1 release nor monocyte recruitment is affected by reduced neutrophil migration after thioglycollate stimulation (170). In this study, although the depletion of resident macrophages resulted in the abrogation of neutrophil infiltration, monocyte recruitment remained unchanged (chapter 3, section 3.2.3). These findings indicate that a macrophage- and neutrophilindependent pathway exists to mediate $\mathrm{MCP}-1$ production within the peritoneum during MSU-induced inflammation.

A possible source of macrophage-/neutrophil-independent MSU-induced MCP-1 production in the model is the surrounding membrane. The role of epithelial cells in leukocyte recruitment has been previously investigated in neoplastic tissue, whereby stromal production of $\mathrm{MCP}-1$ induces tumour-associated macrophage infiltration and contributes to tumour progression (196). In addition, as mentioned in chapter 4 (section 4.1.1.1), synoviocytes isolated from rabbit joints have been shown to produce MCP-1 after LPS stimulation using immunohistochemistry (70). These findings indicate that the local membrane can potentially contribute to the production of MCP-1 resulting in monocyte recruitment during an inflammatory response. The results of chapter 4 section 4.1.2.1 demonstrated that the peritoneal membrane was a major source of MCP-1 after direct MSU 
stimulation. In addition, the production of MCP-1 appears to be mediated in a CD14dependent manner. In vitro culture of primary PMCs isolated from the peritoneum produced high levels of MCP-1 after both MSU crystal and LPS stimulation (chapter 4, section 4.1.2.3). This is consistent with studies by Matsuo and colleagues showing that murine PMCs produce MCP-1 through a TLR4/NFkB-dependent mechanism after LPS challenge (173). Interestingly, cultured PMC production of MCP-1 in response to MSU crystal stimulation appeared to be mediated by a CD14-independent mechanism. This may indicate that in vitro culture had altered the function of the primary cell, allowing the PMCs to lose components of the MSU crystal response pathway. Alternatively, this may suggest that PMCs are not the only source of MCP-1 in vivo. Future directions may involve further characterisation of other $\mathrm{MCP}-1^{+}$cells isolated from the digested peritoneal membrane, or a revisit of direct MCP-1 immunofluorescent histology on whole excised peritoneal tissue to identify cells producing CD14-restricted MCP-1.

In this study, MSU-induced monocyte recruitment remained unaffected even in an inflammatory environment with decreased levels of MCP-1. It is possible that within the local environment, a threshold MCP-1 concentration exists, which once reached will enable the maximum recruitment of circulating monocytes into the peritoneum after MSU administration. Nevertheless, decreased MCP-1 production does indicate a possible role for resident macrophages in the augmentation of $\mathrm{MCP}-1$ production by resident membrane cells. Previous studies have shown that murine peritoneal mesothelium express the IL-1 receptor (197), which may be activated by IL-1 $\beta$ produced by MSU-activated macrophages, and may be responsible for augmenting production of the MCP-1 chemokine by membrane cells.

Another reason for unaffected monocyte recruitment despite a local decrease in MCP-1 levels could be that resident cells may produce mediators of monocyte recruitment other than MCP-1. Fibroblasts and endothelial cells are also able to produce the cytokine macrophage-stimulating factor (M-CSF) (198-200), which has been shown previously to have chemotactic properties $(155,156)$. Transient elevation of M-CSF within the peritoneum after MSU administration was observed in this study (chapter 3, section 3.2.10), indicating a possible role for M-CSF in monocyte recruitment in vivo. In addition, 
thrombin - an enzyme that participates in fibrin formation, has been shown to stimulate production of the chemokine macrophage inflammatory protein-2 (MIP-2) by rat glomerular epithelial cells in vitro (201).

\subsection{Early recruited monocytes are non-responsive to MSU restimulation ex vivo}

Previous in vitro and ex vivo studies have proposed that undifferentiated monocytes are key cells involved in the pro-inflammatory phase of MSU-induced inflammation, and switch to an anti-inflammatory phenotype as they differentiate into macrophages (111113). These studies have shown using monocyte/macrophage cell lines and differentiated human peripheral blood monocytes, that undifferentiated monocytes produce proinflammatory cytokines (such IL-6 and TNF $\alpha$ ) in response to MSU crystal stimulation, whereas mature macrophages do not produce IL-6 and TNF $\alpha(111,112)$. In addition, a study mixing leukocyte populations harvested from human blisters has shown a similar pattern of switching from a "pro"- to an "anti"-inflammatory response when monocytes/macrophages were stimulated with MSU ex vivo (113). However, as these previous studies were based on immortalized murine cells lines and human monocytederived macrophages differentiated over 7 days in vitro, their physiological relevance to the in vivo inflammatory environment remains unclear.

In chapter 3 (section 3.2.8), newly recruited monocytes were shown to be unresponsive to MSU crystal restimulation ex vivo. Contrary to previous in vitro studies, the ability of recruited monocytes/macrophages to produce pro-inflammatory cytokines/chemokines after MSU crystal restimulation developed at later stages of the inflammation profile, coinciding with differentiation towards a macrophage-like phenotype.

This indicated that MSU recognition/activation pathways may be compromised in recruited monocytes and develop during differentiation to macrophages. One such recognition pathway involves the co-factor $\mathrm{CD} 14$, which is believed to be primarily responsible for presenting LPS to TLR4 and subsequent initiation of pro-inflammatory cytokine production via the NFKB pathway $(60,61,202)$. Recombinant soluble CD14 has also been shown to coat the surface of MSU crystals in vitro (54) and appears to facilitate 
the recognition and uptake of MSU crystals by resident phagocytes. Liu-Bryan and colleagues have shown that soluble CD14-coated MSU crystals were able to restore the production of IL-1 $\beta$ in $\mathrm{CD} 14^{-/-}$bone marrow-derived macrophages in vitro (54).

The results in chapter 3 (section 3.2.6) demonstrated that newly recruited monocytes expressed higher levels of CD14 after transmigration through the endothelium; however, these levels were still significantly lower compared to resident macrophages. This may indicate that there are insufficient levels of CD14 on the surface of recruited monocytes to enable recognition of MSU crystals, however responsiveness to LPS restimulation indicated the level of CD14 expression was adequate for functional engagement with the TLR4 pathway. Alternatively it has been shown recently that there are differential requirements for the activation of the NALP3 inflammasome in monocytes and macrophages (203). Therefore, it is possible that CD14 expression is not required for the production of pro-inflammatory cytokines in recruited monocytes after MSU crystal stimulation but becomes a regulatory component as monocytes differentiate into macrophages.

Another possible reason for the lack of response by early recruited monocytes to MSU restimulation could be due to the lower initial phagocytic capacity (chapter 3, section 3.2.9), a function that develops as recruited monocytes differentiate into macrophages. Studies by Mossman and Hansen first observed the phenomenon of "frustrated" phagocytosis with the inability of alveolar macrophages to efficiently phagocytose long thin crystal particles resulting in disrupted plasma membranes and the production of proinflammatory molecules (204). Recently, studies have shown that phagocytosis of crystal structures such as MSU by macrophages results in the subsequent rupture of intracellular lysosomes (56). This process activates the multi-protein complex NALP3 inflammasome that in turn binds and activates procaspase-1 (62). Activated caspase-1 cleaves preformed stores of proIL-1 $\beta$ into active IL-1 $\beta$ allowing it to be secreted from the cell (60). Therefore, stalled development of phagocytic capability may delay the ability of newly recruited monocytes to respond to MSU restimulation. 
The link between low phagocytic capacity and lack of MSU-induced restimulation of newly recruited monocytes may be further supported by the macrophage-depletion model, whereby monocytes recruited into a macrophage-depleted environment exhibited a delay in the development of phagocytic function compared to MSU-recruited monocytes from normal mice. The length of time that recruited monocytes were un-responsive to MSU restimulation correlated with the different rates of development in phagocytic function. While recruited monocytes from normal mice gradually increased responsiveness to MSU crystals after 24 hours, monocytes from macrophage-depleted mice only began to respond after one week.

Future research to confirm the importance of uptake in the recognition of MSU crystals could involve the use of a phagocytosis inhibitor on monocyte/macrophage populations, which should obstruct their responsiveness to MSU crystals thereby suppressing cytokine/chemokine production. Alternatively, as monocytes differentiate into macrophages, the expression and function of the NALP3 inflammasome could be analysed to investigate possible differential response mechanisms to MSU crystals.

\subsection{Recruited monocyte phagocytosis of apoptotic neutrophils and the resolution of MSU-induced inflammation.}

A characteristic feature of a gout attack is the spontaneous resolution of inflammation. Although the cause is not conclusively known, there is indirect evidence to show that mononuclear phagocytes mediate resolution of inflammation in gout through antiinflammatory molecules (including TGF- $\beta)(167,168)$.

In situations where there is delayed neutrophil apoptosis and clearance by macrophages, an accumulation of infiltrating neutrophils within tissues occurs, resulting in recurrent sterile inflammatory complications (152). This has been observed previously in patients with chronic granulomatous disease (CGD), who possess circulating neutrophils that are more resistant to apoptosis in vitro (152). In addition, other studies have shown that is there an increased and prolonged neutrophil influx in $\mathrm{TG}^{-/-}$mice - deficient in macrophage uptake of apoptotic cells (166). These studies indicate that dysfunctional macrophage 
phagocytosis of apoptotic neutrophils can result in unresolved and extended inflammation in vivo. Furthermore, Fadok and colleagues have shown that human monocyte-derived macrophage phagocytosis of apoptotic neutrophils produce anti-inflammatory mediators (including TGF- $\beta$ ) while downregulating pro-inflammatory cytokines (such as TNF $\alpha$ ) in vitro (142). Together these findings indicate that the resolution of inflammation not only requires the removal of apoptotic cells but also the active suppression of the inflammatory environment. However, as these studies have been conducted in vitro, their relevance to the resolution of MSU-induced inflammation in vivo has yet to be demonstrated.

In this study, the peak of neutrophil infiltration at 18 hours after MSU administration in vivo coincided with increased phagocytic ability of differentiated monocytes/macrophages ex vivo (chapter 3, section 3.2.9). When MSU-recruited monocytes were isolated from the infiltrating neutrophils, monocytes produced increased levels of cytokines/chemokines (chapter 3, section 3.2.11). This indicates that the phagocytosis of apoptotic neutrophils by recruited monocytes, may have switched them from a "pro"- to "non"-inflammatory phenotype ex vivo.

In addition to neutrophil uptake, the resolution of inflammation in gout is also thought to involve the anti-inflammatory cytokine TGF- $\beta(113,167)$. Previous studies have shown that administration of recombinant human TGF- $\beta$ abrogated MSU-induced inflammation in a rat subcutaneous air pouch model (110). In addition, Henson and colleagues have shown that anti-TGF- $\beta$ antibody restores cytokine production in LPS-stimulated macrophages that had previously phagocytosed apoptotic cells (142). This indicates a role for TGF- $\beta$ in suppressing the pro-inflammatory capabilities of phagocytes involved in the uptake of apoptotic cells. In this study, direct suppression of monocyte pro-inflammatory capabilities by neutrophil secreted soluble factors was shown when isolated, recruited monocytes were cultured in neutrophilic secretions; this resulted in the suppression of proinflammatory cytokines/chemokines production by the recruited monocytes after MSUrestimulation (chapter 3 , section 3.2.12). In addition, TGF- $\beta$ was shown to play a role in the suppressive effects when TGF- $\beta$ blocking antibody was added to neutrophil and monocyte co-cultures, resulting in the increased production of cytokines/chemokines from the mixed cultures (chapter 3, section 3.2.13). Interestingly, this suppressive effect was 
only observed in later stage recruited pro-inflammatory monocyte/macrophages. However, as early recruited monocytes exhibit no response to MSU crystal restimulation, it is not surprising that TGF- $\beta$ depletion has no real effect on this cell population; supporting the hypothesis that early monocytes may possess dysfunctional pathways in recognising MSU crystals.

Therefore, this phenomenon needs to be examined in further detail, possibly by identifying what soluble factors neutrophils make and then adding each to recruited monocyte cultures to observe suppressive effects. In addition, the importance of TGF- $\beta$ in the initiation and resolution of MSU-induced inflammation could be investigated following the in vivo depletion of TGF- $\beta$. Future research to investigate whether monocytes phagocytose apoptotic neutrophils, contributing to resolution in vivo could involve the i.p. injection of CFSE labelled neutrophils at the peak of MSU-induced inflammation and at different timepoints analysis of monocytes/macrophages for CFSE and monocyte marker co-localisation by flow cytometry.

\subsection{Impact of the inflammatory environment on recruited monocyte differentiation and function}

Until now, there has been very little research into the differentiation and function of monocytes recruited into an acute inflammatory environment $(58,175)$. Even fewer studies have investigated the differences in differentiation and function of recruited monocytes in an abrogated inflammatory environment in vivo. One of the novel findings of this study is the effect of an abrogated acute inflammatory environment on MSUrecruited monocyte differentiation and function. In this model, the process of monocyte to macrophage differentiation is delayed, resulting in functional deviations.

In normal mice, the first 24 hours after monocytes are recruited into an MSU-induced inflammatory environment is characterised by the downregulation of the hemopoietic surface markers Gr-1 and 7/4 (chapter 3, section 3.2.7). At this point, recruited monocytes begin to develop a macrophage-like morphology and upregulate the expression of macrophage surface phenotypes F4/80 and CD11b (macrophage markers), CD206 (mannose receptor) and CD14 (TLR4 co-factor). Although there were no observed 
differences in these surface markers on monocytes isolated from macrophage-depleted compared to normal mice over time, monocytes recruited into the macrophage-depleted environment by MSU administration displayed a delayed development of macrophage morphological phenotypes compared to monocytes from normal mice. Interestingly, there was also a notable decrease in the phagocytic function and capacity to produce proinflammatory cytokines in monocytes isolated from macrophage-depleted compared to normal mice. This indicated a fundamental difference that would have been missed if only cell surface markers were used to determine monocyte differentiation.

Differentiation of recruited monocytes is driven by a variety of molecules; including the hemopoietic-cell growth factors GM-CSF/M-CSF that have been shown to play a key role in driving myeloid cell differentiation (151). The different ratios of CSFs within the inflammatory environment may play a key role in directing the differentiation pathway of recruited monocytes. For example, M-CSF within the tumour environment is believed to play a prominent role in driving the differentiation of recruited monocytes to TAMs by blocking their differentiation into DCs $(96,108)$. Studies by Merad and colleagues has shown that CSF-receptor deficient mice failed to reconstitute the LC pool in inflamed skin in vivo (92). In addition, GM-CSF levels within the bronchoalveolar lavage fluid has been proposed to contribute to monocytes differentiating into a unique DC-like macrophage population (109). Furthermore, an inflammatory environment elicited by bacterial infection appears to preferentially programme recruited monocytes to differentiate into DCs (90).

GM-CSF and M-CSF are two candidate cytokines that were evaluated for their possible effects on recruited monocyte differentiation in this study. During the early phases of MSU-induced inflammation, GM-CSF decreased while M-CSF increased, indicating that the inflammatory microenvironment is positioned towards macrophage differentiation (chapter 3, section 3.2.10). In macrophage-depleted mice, this ratio is altered whereby both GM-CSF and M-CSF levels are abrogated after MSU-stimulation; the decreased MCSF levels may have delayed the programming of monocyte differentiation to macrophages in vivo. Further investigation into the ratios of CSFs within the inflammatory 
environment could include profiling recruited monocyte differentiation in CSF-KO mice or following specific cytokine depletion in vivo.

\subsection{Summary}

The findings presented in this thesis extend the current model of MSU-induced acute inflammation by introducing novel insight into the impact of the microenvironment on the recruitment, differentiation and function of blood monocytes. A schematic representation of my proposed model of MSU-induced inflammation incorporating my findings is presented in Diagram 5.1.

The deposition of MSU crystals within the peritoneum induces the production of MCP-1 from the local membrane. This process may be augmented by the production of IL-1R agonists (such as IL-1 $\beta$ ) produced by activated resident macrophages. In addition, the process of leukocyte recruitment may be mediated by other chemoattractants such as MCSF. As circulating monocytes transmigrate through the endothelium, they display altered expression of surface antigens and low phagocytic capacity, which correlates with a lack of responsiveness to MSU crystal restimulation. The ratio of GM-CSF and M-CSF within the inflammatory environment appears to be responsible for programming monocyte to macrophage differentiation. While not conclusively known, recruited monocytes may contribute to the resolution of acute inflammation by phagocytosing apoptotic neutrophils and producing anti-inflammatory cytokines (such as TGF- $\beta$ ). Furthermore, TGF- $\beta$ secreted by neutrophils appears to contribute to the suppression of overall inflammation. Over the course of a week, recruited monocytes developed phenotypes characteristic of resident macrophages such as phagocytic function and begin to respond to both MSU crystal and LPS restimulation. This indicates that controlled clearance of the existing inflammation may be necessary before the local immune environment can be reset in preparation for future inflammatory insults. 


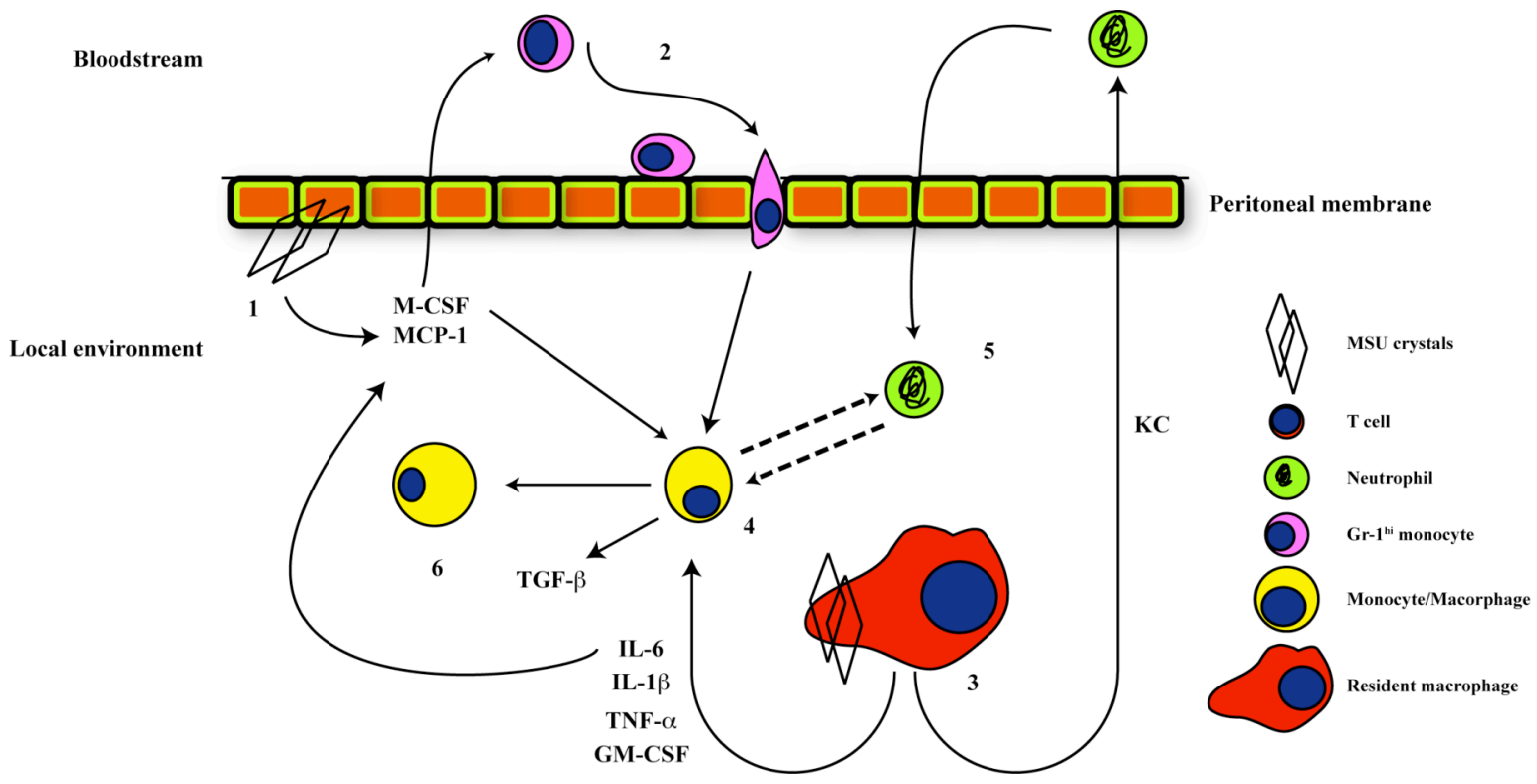

Diagram 5.1: Proposed updates on the MSU-induced inflammation model. 1. Direct exposure of MSU crystals to the peritoneal membrane elicits the production of monocyte chemoattractants MCP-1 and M-CSF. This process is augmented by the inflammatory environment, thereby further increasing chemokine production. 2. Blood monocytes undergo transmigration into the peritoneum and display lower phagocytic capacity and altered surface marker expression. 3. Uptake of MSU crystals by resident macrophages results in the production of inflammatory cytokines including IL-1 $\beta$, IL-6, TNF- $\alpha$ and GMCSF. This creates a highly pro-inflammatory environment into which the blood monocytes are recruited. Activated resident macrophages also produce the chemokine $\mathrm{KC}$, which recruits neutrophils from the bloodstream into the peritoneum. 4. Monocytes recruited into the acute inflammatory environment are nonresponsive to MSU restimulation. Phagocytosis of neutrophils by monocytes switches them into an "anti"inflammatory phenotype able to produce anti-inflammatory cytokines (such as TGF- $\beta$ ). 5 . Infiltrating neutrophils amplify the inflammatory response by producing IL-8 and superoxide. As neutrophils apoptose, they are able to contribute to the resolution of inflammation by secreting soluble factors (including TGF- $\beta$ ). 6. Over the course of a week, recruited monocytes differentiate and develop macrophage-like phenotypes, eventually replenishing the resident macrophage population. 


\section{References}

1. Nuki, G., and P. A. Simkin. 2006. A concise history of gout and hyperuricemia and their treatment. Arthritis Res Ther 8 Suppl 1:S1.

2. Lennane, G. A., B. S. Rose, and I. C. Isdale. 1960. Gout in the Maori. Ann Rheum Dis 19:120-125.

3. Klemp, P., S. A. Stansfield, B. Castle, and M. C. Robertson. 1997. Gout is on the increase in New Zealand. Ann Rheum Dis 56:22-26.

4. Wallace, K. L., A. A. Riedel, N. Joseph-Ridge, and R. Wortmann. 2004. Increasing prevalence of gout and hyperuricemia over 10 years among older adults in a managed care population. J Rheumatol 31:1582-1587.

5. Wallace, S. L., H. Robinson, A. T. Masi, J. L. Decker, D. J. McCarty, and T. F. Yu. 1977. Preliminary criteria for the classification of the acute arthritis of primary gout. Arthritis Rheum 20:895-900.

6. Ferraz, M. B., and B. O'Brien. 1995. A cost effectiveness analysis of urate lowering drugs in nontophaceous recurrent gouty arthritis. $J$ Rheumatol 22:908-914.

7. Nakayama, D. A., C. Barthelemy, G. Carrera, R. W. Lightfoot, Jr., and R. L. Wortmann. 1984. Tophaceous gout: a clinical and radiographic assessment. Arthritis Rheum 27:468-471.

8. Singh, J. A. 2009. Quality of life and quality of care for patients with gout. Curr Rheumatol Rep 11:154-160.

9. Faires, J. S., and D. J. McCarty Jr. 1962. Acute arthritis in man and dog after intrasynovial injection of sodium urate crystals. The Lancet 2:682-685.

10. Pascual, E. 1991. Persistence of monosodium urate crystals and low-grade inflammation in the synovial fluid of patients with untreated gout. Arthritis Rheum 34:141-145.

11. Pascual, E., and V. Jovani. 1995. A quantitative study of the phagocytosis of urate crystals in the synovial fluid of asymptomatic joints of patients with gout. $\mathrm{Br} J$ Rheumatol 34:724-726.

12. Vitart, V., I. Rudan, C. Hayward, N. K. Gray, J. Floyd, C. N. Palmer, S. A. Knott, I. Kolcic, O. Polasek, J. Graessler, J. F. Wilson, A. Marinaki, P. L. Riches, X. Shu, B. Janicijevic, N. Smolej-Narancic, B. Gorgoni, J. Morgan, S. Campbell, Z. Biloglav, L. Barac-Lauc, M. Pericic, I. M. Klaric, L. Zgaga, T. Skaric-Juric, S. H. Wild, W. A. Richardson, P. Hohenstein, C. H. Kimber, A. Tenesa, L. A. Donnelly, L. D. Fairbanks, M. Aringer, P. M. McKeigue, S. H. Ralston, A. D. Morris, P. Rudan, N. D. Hastie, H. Campbell, and A. F. Wright. 2008. SLC2A9 is a newly identified urate transporter influencing serum urate concentration, urate excretion and gout. Nat Genet 40:437-442.

13. Wu, X. W., C. C. Lee, D. M. Muzny, and C. T. Caskey. 1989. Urate oxidase: primary structure and evolutionary implications. Proc Natl Acad Sci USA 86:9412-9416.

14. Wu, X. W., D. M. Muzny, C. C. Lee, and C. T. Caskey. 1992. Two independent mutational events in the loss of urate oxidase during hominoid evolution. $J \mathrm{Mol}$ Evol 34:78-84.

15. Dincer, H. E., A. P. Dincer, and D. J. Levinson. 2002. Asymptomatic hyperuricemia: to treat or not to treat. Cleve Clin J Med 69:594, 597, 600-592 passim. 
16. Campion, E. W., R. J. Glynn, and L. O. DeLabry. 1987. Asymptomatic hyperuricemia. Risks and consequences in the Normative Aging Study. Am J Med 82:421-426.

17. Pascual, E., E. Batlle-Gualda, A. Martinez, J. Rosas, and P. Vela. 1999. Synovial fluid analysis for diagnosis of intercritical gout. Ann Intern Med 131:756-759.

18. Roubenoff, R. 1990. Gout and hyperuricemia. Rheum Dis Clin North Am 16:539550.

19. Hall, A. P., P. E. Barry, T. R. Dawber, and P. M. McNamara. 1967. Epidemiology of gout and hyperuricemia. A long-term population study. Am J Med 42:27-37.

20. Nicholls, A., M. L. Snaith, and J. T. Scott. 1973. Effect of oestrogen therapy on plasma and urinary levels of uric acid. Br Med $J$ 1:449-451.

21. Li, T., J. R. Walsh, F. K. Ghishan, and L. Bai. 2004. Molecular cloning and characterization of a human urate transporter (hURAT1) gene promoter. Biochim Biophys Acta 1681:53-58.

22. Griebsch, A., and N. Zollner. 1974. Effect of ribomononucleotides given orally on uric acid production in man. Adv Exp Med Biol 41:443-449.

23. Coe, F. L., E. Moran, and A. G. Kavalich. 1976. The contribution of dietary purine over-consumption to hyperpuricosuria in calcium oxalate stone formers. J Chronic Dis 29:793-800.

24. Choi, H. K., and G. Curhan. 2008. Soft drinks, fructose consumption, and the risk of gout in men: prospective cohort study. BMJ 336:309-312.

25. Choi, J. W., E. S. Ford, X. Gao, and H. K. Choi. 2008. Sugar-sweetened soft drinks, diet soft drinks, and serum uric acid level: the Third National Health and Nutrition Examination Survey. Arthritis Rheum 59:109-116.

26. Choi, H. K., K. Atkinson, E. W. Karlson, W. Willett, and G. Curhan. 2004. Alcohol intake and risk of incident gout in men: a prospective study. Lancet 363:1277-1281.

27. Choi, H. K., and G. Curhan. 2004. Beer, liquor, and wine consumption and serum uric acid level: the Third National Health and Nutrition Examination Survey. Arthritis Rheum 51:1023-1029.

28. Messerli, F. H., E. D. Frohlich, G. R. Dreslinski, D. H. Suarez, and G. G. Aristimuno. 1980. Serum uric acid in essential hypertension: an indicator of renal vascular involvement. Ann Intern Med 93:817-821.

29. Puig, J. G., and L. M. Ruilope. 1999. Uric acid as a cardiovascular risk factor in arterial hypertension. J Hypertens 17:869-872.

30. Sutton, H. E., and R. P. Wagner. 1975. Mutation and enzyme function in humans. Annu Rev Genet 9:187-212.

31. Ichida, K., M. Hosoyamada, I. Hisatome, A. Enomoto, M. Hikita, H. Endou, and T. Hosoya. 2004. Clinical and molecular analysis of patients with renal hypouricemia in Japan-influence of URAT1 gene on urinary urate excretion. J Am Soc Nephrol 15:164-173.

32. Shima, Y., K. Teruya, and H. Ohta. 2006. Association between intronic SNP in urate-anion exchanger gene, SLC22A12, and serum uric acid levels in Japanese. Life Sci 79:2234-2237.

33. Vazquez-Mellado, J., A. L. Jimenez-Vaca, S. Cuevas-Covarrubias, V. AlvaradoRomano, G. Pozo-Molina, and R. Burgos-Vargas. 2007. Molecular analysis of the SLC22A12 (URAT1) gene in patients with primary gout. Rheumatology (Oxford) 46:215-219.

34. Li, S., S. Sanna, A. Maschio, F. Busonero, G. Usala, A. Mulas, S. Lai, M. Dei, M. Orru, G. Albai, S. Bandinelli, D. Schlessinger, E. Lakatta, A. Scuteri, S. S. Najjar, 
J. Guralnik, S. Naitza, L. Crisponi, A. Cao, G. Abecasis, L. Ferrucci, M. Uda, W. M. Chen, and R. Nagaraja. 2007. The GLUT9 gene is associated with serum uric acid levels in Sardinia and Chianti cohorts. PLoS Genet 3:e194.

35. Stark, K., W. Reinhard, K. Neureuther, S. Wiedmann, K. Sedlacek, A. Baessler, M. Fischer, S. Weber, B. Kaess, J. Erdmann, H. Schunkert, and C. Hengstenberg. 2008. Association of common polymorphisms in GLUT9 gene with gout but not with coronary artery disease in a large case-control study. PLoS ONE 3:e1948.

36. Choi, H. K., K. Atkinson, E. W. Karlson, W. Willett, and G. Curhan. 2004. Purinerich foods, dairy and protein intake, and the risk of gout in men. $N$ Engl J Med 350:1093-1103.

37. Jacob, R. A., G. M. Spinozzi, V. A. Simon, D. S. Kelley, R. L. Prior, B. HessPierce, and A. A. Kader. 2003. Consumption of cherries lowers plasma urate in healthy women. J Nutr 133:1826-1829.

38. Huang, H. Y., L. J. Appel, M. J. Choi, A. C. Gelber, J. Charleston, E. P. Norkus, and E. R. Miller, 3rd. 2005. The effects of vitamin C supplementation on serum concentrations of uric acid: results of a randomized controlled trial. Arthritis Rheum 52:1843-1847.

39. Choi, H. K., W. Willett, and G. Curhan. 2007. Coffee consumption and risk of incident gout in men: a prospective study. Arthritis Rheum 56:2049-2055.

40. Di Giovine, F. S., S. E. Malawista, G. Nuki, and G. W. Duff. 1987. Interleukin 1 (IL 1) as a mediator of crystal arthritis. Stimulation of T cell and synovial fibroblast mitogenesis by urate crystal-induced IL 1. J Immunol 138:3213-3218.

41. di Giovine, F. S., S. E. Malawista, E. Thornton, and G. W. Duff. 1991. Urate crystals stimulate production of tumor necrosis factor alpha from human blood monocytes and synovial cells. Cytokine mRNA and protein kinetics, and cellular distribution. J Clin Invest 87:1375-1381.

42. Getting, S. J., R. J. Flower, L. Parente, R. de Medicis, A. Lussier, B. A. Woliztky, M. A. Martins, and M. Perretti. 1997. Molecular determinants of monosodium urate crystal-induced murine peritonitis: a role for endogenous mast cells and a distinct requirement for endothelial-derived selectins. J Pharmacol Exp Ther 283:123-130.

43. Guerne, P. A., R. Terkeltaub, B. Zuraw, and M. Lotz. 1989. Inflammatory microcrystals stimulate interleukin-6 production and secretion by human monocytes and synoviocytes. Arthritis Rheum 32:1443-1452.

44. Russell, I. J., C. Mansen, L. M. Kolb, and W. P. Kolb. 1982. Activation of the fifth component of human complement (C5) induced by monosodium urate crystals: C5 convertase assembly on the crystal surface. Clin Immunol Immunopathol 24:239250.

45. Ryckman, C., C. Gilbert, R. de Medicis, A. Lussier, K. Vandal, and P. A. Tessier. 2004. Monosodium urate monohydrate crystals induce the release of the proinflammatory protein S100A8/A9 from neutrophils. J Leukoc Biol 76:433-440.

46. Abramson, S., S. T. Hoffstein, and G. Weissmann. 1982. Superoxide anion generation by human neutrophils exposed to monosodium urate. Arthritis Rheum 25:174-180.

47. Matzinger, P. 1994. Tolerance, danger, and the extended family. Annu Rev Immunol 12:991-1045.

48. Akira, S., K. Takeda, and T. Kaisho. 2001. Toll-like receptors: critical proteins linking innate and acquired immunity. Nat Immunol 2:675-680. 
49. Shi, Y., W. Zheng, and K. L. Rock. 2000. Cell injury releases endogenous adjuvants that stimulate cytotoxic T cell responses. Proc Natl Acad Sci U S A 97:14590-14595.

50. Shi, Y., and K. L. Rock. 2002. Cell death releases endogenous adjuvants that selectively enhance immune surveillance of particulate antigens. Eur J Immunol 32:155-162.

51. Shi, Y., J. E. Evans, and K. L. Rock. 2003. Molecular identification of a danger signal that alerts the immune system to dying cells. Nature 425:516-521.

52. Tramontini, N., C. Huber, R. Liu-Bryan, R. A. Terkeltaub, and K. S. Kilgore. 2004. Central role of complement membrane attack complex in monosodium urate crystal-induced neutrophilic rabbit knee synovitis. Arthritis Rheum 50:2633-2639.

53. Liu-Bryan, R., P. Scott, A. Sydlaske, D. M. Rose, and R. Terkeltaub. 2005. Innate immunity conferred by Toll-like receptors 2 and 4 and myeloid differentiation factor 88 expression is pivotal to monosodium urate monohydrate crystal-induced inflammation. Arthritis Rheum 52:2936-2946.

54. Scott, P., H. Ma, S. Viriyakosol, R. Terkeltaub, and R. Liu-Bryan. 2006. Engagement of CD14 mediates the inflammatory potential of monosodium urate crystals. J Immunol 177:6370-6378.

55. Chen, C. J., Y. Shi, A. Hearn, K. Fitzgerald, D. Golenbock, G. Reed, S. Akira, and K. L. Rock. 2006. MyD88-dependent IL-1 receptor signaling is essential for gouty inflammation stimulated by monosodium urate crystals. J Clin Invest 116:22622271.

56. Hornung, V., F. Bauernfeind, A. Halle, E. O. Samstad, H. Kono, K. L. Rock, K. A. Fitzgerald, and E. Latz. 2008. Silica crystals and aluminum salts activate the NALP3 inflammasome through phagosomal destabilization. Nat Immunol 9:847856.

57. Gordon, S., and P. R. Taylor. 2005. Monocyte and macrophage heterogeneity. Nat Rev Immunol 5:953-964.

58. Martin, W. J., M. Walton, and J. Harper. 2009. Resident macrophages initiating and driving inflammation in a monosodium urate monohydrate crystal-induced murine peritoneal model of acute gout. Arthritis Rheum 60:281-289.

59. Jaramillo, M., P. H. Naccache, and M. Olivier. 2004. Monosodium urate crystals synergize with IFN-gamma to generate macrophage nitric oxide: involvement of extracellular signal-regulated kinase 1/2 and NF-kappa B. J Immunol 172:57345742.

60. Martinon, F., V. Petrilli, A. Mayor, A. Tardivel, and J. Tschopp. 2006. Goutassociated uric acid crystals activate the NALP3 inflammasome. Nature 440:237241.

61. Petrilli, V., C. Dostert, D. A. Muruve, and J. Tschopp. 2007. The inflammasome: a danger sensing complex triggering innate immunity. Curr Opin Immunol 19:615622.

62. Mariathasan, S., K. Newton, D. M. Monack, D. Vucic, D. M. French, W. P. Lee, M. Roose-Girma, S. Erickson, and V. M. Dixit. 2004. Differential activation of the inflammasome by caspase-1 adaptors ASC and Ipaf. Nature 430:213-218.

63. Lee, J., G. Cacalano, T. Camerato, K. Toy, M. W. Moore, and W. I. Wood. 1995. Chemokine binding and activities mediated by the mouse IL-8 receptor. J Immunol 155:2158-2164.

64. Terkeltaub, R., S. Baird, P. Sears, R. Santiago, and W. Boisvert. 1998. The murine homolog of the interleukin-8 receptor CXCR-2 is essential for the occurrence of 
neutrophilic inflammation in the air pouch model of acute urate crystal-induced gouty synovitis. Arthritis Rheum 41:900-909.

65. Karin, M. 2005. Inflammation and cancer: the long reach of Ras. Nat Med 11:2021.

66. Hachicha, M., P. H. Naccache, and S. R. McColl. 1995. Inflammatory microcrystals differentially regulate the secretion of macrophage inflammatory protein 1 and interleukin 8 by human neutrophils: a possible mechanism of neutrophil recruitment to sites of inflammation in synovitis. $J$ Exp Med 182:20192025.

67. Ryckman, C., S. R. McColl, K. Vandal, R. de Medicis, A. Lussier, P. E. Poubelle, and P. A. Tessier. 2003. Role of S100A8 and S100A9 in neutrophil recruitment in response to monosodium urate monohydrate crystals in the air-pouch model of acute gouty arthritis. Arthritis Rheum 48:2310-2320.

68. Chapman, P. T., H. Yarwood, A. A. Harrison, C. J. Stocker, F. Jamar, R. H. Gundel, A. M. Peters, and D. O. Haskard. 1997. Endothelial activation in monosodium urate monohydrate crystal-induced inflammation: in vitro and in vivo studies on the roles of tumor necrosis factor alpha and interleukin-1. Arthritis Rheum 40:955-965.

69. Jaramillo, M., M. Godbout, P. H. Naccache, and M. Olivier. 2004. Signaling events involved in macrophage chemokine expression in response to monosodium urate crystals. J Biol Chem 279:52797-52805.

70. Matsukawa, A., S. Miyazaki, T. Maeda, S. Tanase, L. Feng, S. Ohkawara, M. Yoshinaga, and T. Yoshimura. 1998. Production and regulation of monocyte chemoattractant protein-1 in lipopolysaccharide- or monosodium urate crystalinduced arthritis in rabbits: roles of tumor necrosis factor alpha, interleukin-1, and interleukin-8. Lab Invest 78:973-985.

71. Ley, K., C. Laudanna, M. I. Cybulsky, and S. Nourshargh. 2007. Getting to the site of inflammation: the leukocyte adhesion cascade updated. Nat Rev Immunol 7:678689.

72. McEver, R. P., and R. D. Cummings. 1997. Role of PSGL-1 binding to selectins in leukocyte recruitment. J Clin Invest 100:S97-103.

73. Iwamoto, T., H. Okamoto, Y. Toyama, and S. Momohara. 2008. Molecular aspects of rheumatoid arthritis: chemokines in the joints of patients. FEBS $J$ 275:44484455.

74. Hyduk, S. J., J. R. Chan, S. T. Duffy, M. Chen, M. D. Peterson, T. K. Waddell, G. C. Digby, K. Szaszi, A. Kapus, and M. I. Cybulsky. 2007. Phospholipase C, calcium, and calmodulin are critical for alpha4beta1 integrin affinity up-regulation and monocyte arrest triggered by chemoattractants. Blood 109:176-184.

75. Campbell, J. J., S. Qin, K. B. Bacon, C. R. Mackay, and E. C. Butcher. 1996. Biology of chemokine and classical chemoattractant receptors: differential requirements for adhesion-triggering versus chemotactic responses in lymphoid cells. J Cell Biol 134:255-266.

76. Campbell, J. J., J. Hedrick, A. Zlotnik, M. A. Siani, D. A. Thompson, and E. C. Butcher. 1998. Chemokines and the arrest of lymphocytes rolling under flow conditions. Science 279:381-384.

77. Schenkel, A. R., Z. Mamdouh, and W. A. Muller. 2004. Locomotion of monocytes on endothelium is a critical step during extravasation. Nat Immunol 5:393-400. 
78. Feng, D., J. A. Nagy, K. Pyne, H. F. Dvorak, and A. M. Dvorak. 1998. Neutrophils emigrate from venules by a transendothelial cell pathway in response to FMLP. $J$ Exp Med 187:903-915.

79. Engelhardt, B., and H. Wolburg. 2004. Mini-review: Transendothelial migration of leukocytes: through the front door or around the side of the house? Eur J Immunol 34:2955-2963.

80. Athens, J. W., O. P. Haab, S. O. Raab, A. M. Mauer, H. Ashenbrucker, G. E. Cartwright, and M. M. Wintrobe. 1961. Leukokinetic studies. IV. The total blood, circulating and marginal granulocyte pools and the granulocyte turnover rate in normal subjects. J Clin Invest 40:989-995.

81. Dancey, J. T., K. A. Deubelbeiss, L. A. Harker, and C. A. Finch. 1976. Neutrophil kinetics in man. $J$ Clin Invest 58:705-715.

82. Shi, J., G. E. Gilbert, Y. Kokubo, and T. Ohashi. 2001. Role of the liver in regulating numbers of circulating neutrophils. Blood 98:1226-1230.

83. Cassatella, M. A. 1995. The production of cytokines by polymorphonuclear neutrophils. Immunol Today 16:21-26.

84. Ozinsky, A., D. M. Underhill, J. D. Fontenot, A. M. Hajjar, K. D. Smith, C. B. Wilson, L. Schroeder, and A. Aderem. 2000. The repertoire for pattern recognition of pathogens by the innate immune system is defined by cooperation between tolllike receptors. Proc Natl Acad Sci U S A 97:13766-13771.

85. Tjelle, T. E., T. Lovdal, and T. Berg. 2000. Phagosome dynamics and function. Bioessays 22:255-263.

86. Hampton, M. B., A. J. Kettle, and C. C. Winterbourn. 1998. Inside the neutrophil phagosome: oxidants, myeloperoxidase, and bacterial killing. Blood 92:3007-3017.

87. Harada, A., N. Sekido, T. Akahoshi, T. Wada, N. Mukaida, and K. Matsushima. 1994. Essential involvement of interleukin-8 (IL-8) in acute inflammation. $J$ Leukoc Biol 56:559-564.

88. Akgul, C., D. A. Moulding, and S. W. Edwards. 2001. Molecular control of neutrophil apoptosis. FEBS Lett 487:318-322.

89. Gordon, S. 2007. The macrophage: past, present and future. Eur J Immunol 37 Suppl 1:S9-17.

90. Auffray, C., D. Fogg, M. Garfa, G. Elain, O. Join-Lambert, S. Kayal, S. Sarnacki, A. Cumano, G. Lauvau, and F. Geissmann. 2007. Monitoring of blood vessels and tissues by a population of monocytes with patrolling behavior. Science 317:666670.

91. Geissmann, F., S. Jung, and D. R. Littman. 2003. Blood monocytes consist of two principal subsets with distinct migratory properties. Immunity 19:71-82.

92. Ginhoux, F., F. Tacke, V. Angeli, M. Bogunovic, M. Loubeau, X. M. Dai, E. R. Stanley, G. J. Randolph, and M. Merad. 2006. Langerhans cells arise from monocytes in vivo. Nat Immunol 7:265-273.

93. Arnold, L., A. Henry, F. Poron, Y. Baba-Amer, N. van Rooijen, A. Plonquet, R. K. Gherardi, and B. Chazaud. 2007. Inflammatory monocytes recruited after skeletal muscle injury switch into antiinflammatory macrophages to support myogenesis. $J$ Exp Med 204:1057-1069.

94. van Furth, R., and Z. A. Cohn. 1968. The origin and kinetics of mononuclear phagocytes. J Exp Med 128:415-435.

95. Edwards, J. P., X. Zhang, K. A. Frauwirth, and D. M. Mosser. 2006. Biochemical and functional characterization of three activated macrophage populations. $J$ Leukoc Biol 80:1298-1307. 
96. Mantovani, A., S. Sozzani, M. Locati, P. Allavena, and A. Sica. 2002. Macrophage polarization: tumor-associated macrophages as a paradigm for polarized M2 mononuclear phagocytes. Trends Immunol 23:549-555.

97. Pestka, S., C. D. Krause, D. Sarkar, M. R. Walter, Y. Shi, and P. B. Fisher. 2004. Interleukin-10 and related cytokines and receptors. Annu Rev Immunol 22:929-979.

98. Derynck, R., R. J. Akhurst, and A. Balmain. 2001. TGF-beta signaling in tumor suppression and cancer progression. Nat Genet 29:117-129.

99. Wohler, J. E., S. S. Smith, K. R. Zinn, D. C. Bullard, and S. R. Barnum. 2009. gammadelta $\mathrm{T}$ cells in EAE: Early trafficking events and cytokine requirements. Eur J Immunol.

100. Komiyama, Y., S. Nakae, T. Matsuki, A. Nambu, H. Ishigame, S. Kakuta, K. Sudo, and Y. Iwakura. 2006. IL-17 plays an important role in the development of experimental autoimmune encephalomyelitis. J Immunol 177:566-573.

101. O'Connor, R. A., C. T. Prendergast, C. A. Sabatos, C. W. Lau, M. D. Leech, D. C. Wraith, and S. M. Anderton. 2008. Cutting edge: Th1 cells facilitate the entry of Th17 cells to the central nervous system during experimental autoimmune encephalomyelitis. J Immunol 181:3750-3754.

102. Deshpande, P., I. L. King, and B. M. Segal. 2007. Cutting edge: CNS CD11c+ cells from mice with encephalomyelitis polarize Th17 cells and support CD25+CD4+ T cell-mediated immunosuppression, suggesting dual roles in the disease process. $J$ Immunol 178:6695-6699.

103. Segal, B. M. 2005. CNS chemokines, cytokines, and dendritic cells in autoimmune demyelination. $J$ Neurol Sci 228:210-214.

104. Bar-Or, A., E. M. Oliveira, D. E. Anderson, and D. A. Hafler. 1999. Molecular pathogenesis of multiple sclerosis. J Neuroimmunol 100:252-259.

105. Warren, M. K., and S. N. Vogel. 1985. Bone marrow-derived macrophages: development and regulation of differentiation markers by colony-stimulating factor and interferons. J Immunol 134:982-989.

106. Zhou, L. J., and T. F. Tedder. 1996. CD14+ blood monocytes can differentiate into functionally mature CD83+ dendritic cells. Proc Natl Acad Sci U S A 93:25882592.

107. Udagawa, N., N. Takahashi, T. Akatsu, H. Tanaka, T. Sasaki, T. Nishihara, T. Koga, T. J. Martin, and T. Suda. 1990. Origin of osteoclasts: mature monocytes and macrophages are capable of differentiating into osteoclasts under a suitable microenvironment prepared by bone marrow-derived stromal cells. Proc Natl Acad Sci U S A 87:7260-7264.

108. Allavena, P., A. Sica, A. Vecchi, M. Locati, S. Sozzani, and A. Mantovani. 2000. The chemokine receptor switch paradigm and dendritic cell migration: its significance in tumor tissues. Immunol Rev 177:141-149.

109. Guth, A. M., W. J. Janssen, C. M. Bosio, E. C. Crouch, P. M. Henson, and S. W. Dow. 2009. Lung environment determines unique phenotype of alveolar macrophages. Am J Physiol Lung Cell Mol Physiol 296:L936-946.

110. Liote, F., F. Prudhommeaux, C. Schiltz, R. Champy, A. Herbelin, E. Ortiz-Bravo, and T. Bardin. 1996. Inhibition and prevention of monosodium urate monohydrate crystal-induced acute inflammation in vivo by transforming growth factor betal. Arthritis Rheum 39:1192-1198.

111. Yagnik, D. R., P. Hillyer, D. Marshall, C. D. Smythe, T. Krausz, D. O. Haskard, and R. C. Landis. 2000. Noninflammatory phagocytosis of monosodium urate 
monohydrate crystals by mouse macrophages. Implications for the control of joint inflammation in gout. Arthritis Rheum 43:1779-1789.

112. Landis, R. C., D. R. Yagnik, O. Florey, P. Philippidis, V. Emons, J. C. Mason, and D. O. Haskard. 2002. Safe disposal of inflammatory monosodium urate monohydrate crystals by differentiated macrophages. Arthritis Rheum 46:30263033.

113. Yagnik, D. R., B. J. Evans, O. Florey, J. C. Mason, R. C. Landis, and D. O. Haskard. 2004. Macrophage release of transforming growth factor betal during resolution of monosodium urate monohydrate crystal-induced inflammation. Arthritis Rheum 50:2273-2280.

114. Thomas-Ecker, S., A. Lindecke, W. Hatzmann, C. Kaltschmidt, K. S. Zanker, and T. Dittmar. 2007. Alteration in the gene expression pattern of primary monocytes after adhesion to endothelial cells. Proc Natl Acad Sci U S A 104:5539-5544.

115. Fleetwood, A. J., T. Lawrence, J. A. Hamilton, and A. D. Cook. 2007. Granulocyte-macrophage colony-stimulating factor (CSF) and macrophage CSFdependent macrophage phenotypes display differences in cytokine profiles and transcription factor activities: implications for CSF blockade in inflammation. $J$ Immunol 178:5245-5252.

116. Tipping, P. G. 2006. Toll-like receptors: the interface between innate and adaptive immunity. J Am Soc Nephrol 17:1769-1771.

117. Kim, K. D., J. Zhao, S. Auh, X. Yang, P. Du, H. Tang, and Y. X. Fu. 2007. Adaptive immune cells temper initial innate responses. Nat Med 13:1248-1252.

118. Guarda, G., C. Dostert, F. Staehli, K. Cabalzar, R. Castillo, A. Tardivel, P. Schneider, and J. Tschopp. 2009. T cells dampen innate immune responses through inhibition of NLRP1 and NLRP3 inflammasomes. Nature.

119. Youn, J. I., S. Nagaraj, M. Collazo, and D. I. Gabrilovich. 2008. Subsets of myeloid-derived suppressor cells in tumor-bearing mice. J Immunol 181:57915802.

120. Barry, M., and R. C. Bleackley. 2002. Cytotoxic T lymphocytes: all roads lead to death. Nat Rev Immunol 2:401-409.

121. Abbas, A. K., K. M. Murphy, and A. Sher. 1996. Functional diversity of helper T lymphocytes. Nature 383:787-793.

122. Min, B., H. Yamane, J. Hu-Li, and W. E. Paul. 2005. Spontaneous and homeostatic proliferation of CD4 $\mathrm{T}$ cells are regulated by different mechanisms. J Immunol 174:6039-6044.

123. Matsumoto, M., M. Sugimoto, H. Nakashima, F. Imamura, O. Kawano, E. Uyama, K. Takatsu, and S. Araki. 1990. Spontaneous T cell proliferation and release of soluble interleukin-2 receptors in patients with HTLV-I-associated myelopathy. Am J Trop Med Hyg 42:365-373.

124. Prince, H. E., J. York, J. Golding, S. M. Owen, and R. B. Lal. 1994. Spontaneous lymphocyte proliferation in human T-cell lymphotropic virus type I (HTLV-I) and HTLV-II infection: T-cell subset responses and their relationships to the presence of provirus and viral antigen production. Clin Diagn Lab Immunol 1:273-282.

125. Schiltz, C., F. Liote, F. Prudhommeaux, A. Meunier, R. Champy, J. Callebert, and T. Bardin. 2002. Monosodium urate monohydrate crystal-induced inflammation in vivo: quantitative histomorphometric analysis of cellular events. Arthritis Rheum 46:1643-1650.

126. Bot, J., D. Whitaker, J. Vivian, R. Lake, V. Yao, and R. McCauley. 2003. Culturing mouse peritoneal mesothelial cells. Pathol Res Pract 199:341-344. 
127. Muller, J., and T. Yoshida. 1995. Interaction of murine peritoneal leukocytes and mesothelial cells: in vitro model system to survey cellular events on serosal membranes during inflammation. Clin Immunol Immunopathol 75:231-238.

128. Yamaji, K., K. Ohnishi, R. Zuinen, Y. Ochiai, T. Chikuma, and H. Hojo. 2008. Interleukin-6 production by peritoneal mesothelial cells and its regulation by inflammatory factors in rats administered carbon tetrachloride intraperitoneally. Toxicol Appl Pharmacol 226:38-45.

129. Van Rooijen, N., and A. Sanders. 1994. Liposome mediated depletion of macrophages: mechanism of action, preparation of liposomes and applications. $J$ Immunol Methods 174:83-93.

130. Scarpino, S., A. Stoppacciaro, F. Ballerini, M. Marchesi, M. Prat, M. C. Stella, S. Sozzani, P. Allavena, A. Mantovani, and L. P. Ruco. 2000. Papillary carcinoma of the thyroid: hepatocyte growth factor (HGF) stimulates tumor cells to release chemokines active in recruiting dendritic cells. Am J Pathol 156:831-837.

131. Nowicki, A., J. Szenajch, G. Ostrowska, A. Wojtowicz, K. Wojtowicz, A. A. Kruszewski, M. Maruszynski, S. L. Aukerman, and W. Wiktor-Jedrzejczak. 1996. Impaired tumor growth in colony-stimulating factor 1 (CSF-1)-deficient, macrophage-deficient op/op mouse: evidence for a role of CSF-1-dependent macrophages in formation of tumor stroma. Int J Cancer 65:112-119.

132. Yamamoto, Y., H. Ishigaki, H. Ishida, Y. Itoh, Y. Noda, and K. Ogasawara. 2008. Analysis of splenic Gr-1int immature myeloid cells in tumor-bearing mice. Microbiol Immunol 52:47-53.

133. Almand, B., J. I. Clark, E. Nikitina, J. van Beynen, N. R. English, S. C. Knight, D. P. Carbone, and D. I. Gabrilovich. 2001. Increased production of immature myeloid cells in cancer patients: a mechanism of immunosuppression in cancer. $J$ Immunol 166:678-689.

134. Kusmartsev, S., S. Nagaraj, and D. I. Gabrilovich. 2005. Tumor-associated CD8+ T cell tolerance induced by bone marrow-derived immature myeloid cells. J Immunol 175:4583-4592.

135. Melani, C., C. Chiodoni, G. Forni, and M. P. Colombo. 2003. Myeloid cell expansion elicited by the progression of spontaneous mammary carcinomas in cerbB-2 transgenic BALB/c mice suppresses immune reactivity. Blood 102:21382145.

136. King, I. L., T. L. Dickendesher, and B. M. Segal. 2009. Circulating Ly-6C+ myeloid precursors migrate to the CNS and play a pathogenic role during autoimmune demyelinating disease. Blood 113:3190-3197.

137. Zhu, B., Y. Bando, S. Xiao, K. Yang, A. C. Anderson, V. K. Kuchroo, and S. J. Khoury. 2007. CD11b+Ly-6C(hi) suppressive monocytes in experimental autoimmune encephalomyelitis. J Immunol 179:5228-5237.

138. Gabrilovich, D. I., V. Bronte, S. H. Chen, M. P. Colombo, A. Ochoa, S. OstrandRosenberg, and H. Schreiber. 2007. The terminology issue for myeloid-derived suppressor cells. Cancer Res 67:425; author reply 426.

139. Dalbeth, N., and D. O. Haskard. 2005. Mechanisms of inflammation in gout. Rheumatology (Oxford) 44:1090-1096.

140. Martinon, F., and L. H. Glimcher. 2006. Gout: new insights into an old disease. $J$ Clin Invest 116:2073-2075.

141. Sunderkotter, C., T. Nikolic, M. J. Dillon, N. Van Rooijen, M. Stehling, D. A. Drevets, and P. J. Leenen. 2004. Subpopulations of mouse blood monocytes differ in maturation stage and inflammatory response. J Immunol 172:4410-4417. 
142. Fadok, V. A., D. L. Bratton, A. Konowal, P. W. Freed, J. Y. Westcott, and P. M. Henson. 1998. Macrophages that have ingested apoptotic cells in vitro inhibit proinflammatory cytokine production through autocrine/paracrine mechanisms involving TGF-beta, PGE2, and PAF. J Clin Invest 101:890-898.

143. Savill, J. S., A. H. Wyllie, J. E. Henson, M. J. Walport, P. M. Henson, and C. Haslett. 1989. Macrophage phagocytosis of aging neutrophils in inflammation. Programmed cell death in the neutrophil leads to its recognition by macrophages. $J$ Clin Invest 83:865-875.

144. Galeazzi, F., E. M. Haapala, N. van Rooijen, B. A. Vallance, and S. M. Collins. 2000. Inflammation-induced impairment of enteric nerve function in nematodeinfected mice is macrophage dependent. Am J Physiol Gastrointest Liver Physiol 278:G259-265.

145. Naito, M., H. Nagai, S. Kawano, H. Umezu, H. Zhu, H. Moriyama, T. Yamamoto, H. Takatsuka, and Y. Takei. 1996. Liposome-encapsulated dichloromethylene diphosphonate induces macrophage apoptosis in vivo and in vitro. $J$ Leukoc Biol 60:337-344.

146. Monkkonen, H., M. J. Rogers, N. Makkonen, S. Niva, S. Auriola, and J. Monkkonen. 2001. The cellular uptake and metabolism of clodronate in RAW 264 macrophages. Pharm Res 18:1550-1555.

147. Monkkonen, J., and T. D. Heath. 1993. The effects of liposome-encapsulated and free clodronate on the growth of macrophage-like cells in vitro: the role of calcium and iron. Calcif Tissue Int 53:139-146.

148. van Rooijen, N., A. Sanders, and T. K. van den Berg. 1996. Apoptosis of macrophages induced by liposome-mediated intracellular delivery of clodronate and propamidine. J Immunol Methods 193:93-99.

149. Fleisch, H. 1987. Bisphosphonates--history and experimental basis. Bone 8 Suppl 1:S23-28.

150. Schmidt-Weber, C. B., M. Rittig, E. Buchner, I. Hauser, I. Schmidt, E. PalomboKinne, F. Emmrich, and R. W. Kinne. 1996. Apoptotic cell death in activated monocytes following incorporation of clodronate-liposomes. J Leukoc Biol 60:230244.

151. Hamilton, J. A. 2008. Colony-stimulating factors in inflammation and autoimmunity. Nat Rev Immunol 8:533-544.

152. Brown, J. R., D. Goldblatt, J. Buddle, L. Morton, and A. J. Thrasher. 2003. Diminished production of anti-inflammatory mediators during neutrophil apoptosis and macrophage phagocytosis in chronic granulomatous disease (CGD). J Leukoc Biol 73:591-599.

153. Soehnlein, O., Y. Kai-Larsen, R. Frithiof, O. E. Sorensen, E. Kenne, K. Scharffetter-Kochanek, E. E. Eriksson, H. Herwald, B. Agerberth, and L. Lindbom. 2008. Neutrophil primary granule proteins HBP and HNP1-3 boost bacterial phagocytosis by human and murine macrophages. J Clin Invest 118:3491-3502.

154. Sherry, B., P. Tekamp-Olson, C. Gallegos, D. Bauer, G. Davatelis, S. D. Wolpe, F. Masiarz, D. Coit, and A. Cerami. 1988. Resolution of the two components of macrophage inflammatory protein 1 , and cloning and characterization of one of those components, macrophage inflammatory protein 1 beta. $J$ Exp Med 168:22512259.

155. Fuller, K., J. M. Owens, C. J. Jagger, A. Wilson, R. Moss, and T. J. Chambers. 1993. Macrophage colony-stimulating factor stimulates survival and chemotactic behavior in isolated osteoclasts. J Exp Med 178:1733-1744. 
156. Pierce, J. H., E. Di Marco, G. W. Cox, D. Lombardi, M. Ruggiero, L. Varesio, L. M. Wang, G. G. Choudhury, A. Y. Sakaguchi, P. P. Di Fiore, and et al. 1990. Macrophage-colony-stimulating factor (CSF-1) induces proliferation, chemotaxis, and reversible monocytic differentiation in myeloid progenitor cells transfected with the human c-fms/CSF-1 receptor cDNA. Proc Natl Acad Sci U S A 87:56135617.

157. Frye, C. A., D. E. Yocum, R. Tuan, E. Suyana, E. A. Seftor, R. E. Seftor, Z. Khalkhali-Ellis, T. L. Moore, and M. J. Hendrix. 1996. An in vitro Model for Studying Mechanisms Underlying Synoviocyte-Mediated Cartilage Invasion in Rheumatoid Arthritis. Pathol Oncol Res 2:157-166.

158. Li, X., Z. Wen, X. He, and S. He. 2006. Effects of cinnamic acid on expression of tissue factor induced by TNFalpha in endothelial cells and its mechanisms. $J$ Chin Med Assoc 69:207-212.

159. Dostert, C., V. Petrilli, R. Van Bruggen, C. Steele, B. T. Mossman, and J. Tschopp. 2008. Innate immune activation through Nalp3 inflammasome sensing of asbestos and silica. Science 320:674-677.

160. Lari, R., A. J. Fleetwood, P. D. Kitchener, A. D. Cook, D. Pavasovic, P. J. Hertzog, and J. A. Hamilton. 2007. Macrophage lineage phenotypes and osteoclastogenesis-complexity in the control by GM-CSF and TGF-beta. Bone 40:323-336.

161. Willman, C. L., C. C. Stewart, V. Miller, T. L. Yi, and T. B. Tomasi. 1989. Regulation of MHC class II gene expression in macrophages by hematopoietic colony-stimulating factors (CSF). Induction by granulocyte/macrophage CSF and inhibition by CSF-1. J Exp Med 170:1559-1567.

162. Menetrier-Caux, C., G. Montmain, M. C. Dieu, C. Bain, M. C. Favrot, C. Caux, and J. Y. Blay. 1998. Inhibition of the differentiation of dendritic cells from CD34(+) progenitors by tumor cells: role of interleukin- 6 and macrophage colonystimulating factor. Blood 92:4778-4791.

163. Dauer, M., K. Schad, J. Junkmann, C. Bauer, J. Herten, R. Kiefl, M. Schnurr, S. Endres, and A. Eigler. 2006. IFN-alpha promotes definitive maturation of dendritic cells generated by short-term culture of monocytes with GM-CSF and IL-4. $J$ Leukoc Biol 80:278-286.

164. Weigel, B. J., N. Nath, P. A. Taylor, A. Panoskaltsis-Mortari, W. Chen, A. M. Krieg, K. Brasel, and B. R. Blazar. 2002. Comparative analysis of murine marrowderived dendritic cells generated by Flt3L or GM-CSF/IL-4 and matured with immune stimulatory agents on the in vivo induction of antileukemia responses. Blood 100:4169-4176.

165. Soehnlein, O., A. Zernecke, E. E. Eriksson, A. G. Rothfuchs, C. T. Pham, H. Herwald, K. Bidzhekov, M. E. Rottenberg, C. Weber, and L. Lindbom. 2008. Neutrophil secretion products pave the way for inflammatory monocytes. Blood 112:1461-1471.

166. Rose, D. M., A. D. Sydlaske, A. Agha-Babakhani, K. Johnson, and R. Terkeltaub. 2006. Transglutaminase 2 limits murine peritoneal acute gout-like inflammation by regulating macrophage clearance of apoptotic neutrophils. Arthritis Rheum 54:3363-3371.

167. Huynh, M. L., V. A. Fadok, and P. M. Henson. 2002. Phosphatidylserinedependent ingestion of apoptotic cells promotes TGF-betal secretion and the resolution of inflammation. J Clin Invest 109:41-50. 
168. Grotendorst, G. R., G. Smale, and D. Pencev. 1989. Production of transforming growth factor beta by human peripheral blood monocytes and neutrophils. $J$ Cell Physiol 140:396-402.

169. Doherty, D. E., G. P. Downey, G. S. Worthen, C. Haslett, and P. M. Henson. 1988. Monocyte retention and migration in pulmonary inflammation. Requirement for neutrophils. Lab Invest 59:200-213.

170. Henderson, R. B., J. A. Hobbs, M. Mathies, and N. Hogg. 2003. Rapid recruitment of inflammatory monocytes is independent of neutrophil migration. Blood 102:328335.

171. Mutsaers, S. E. 2002. Mesothelial cells: their structure, function and role in serosal repair. Respirology 7:171-191.

172. Robson, R. L., R. M. McLoughlin, J. Witowski, P. Loetscher, T. S. Wilkinson, S. A. Jones, and N. Topley. 2001. Differential regulation of chemokine production in human peritoneal mesothelial cells: IFN-gamma controls neutrophil migration across the mesothelium in vitro and in vivo. J Immunol 167:1028-1038.

173. Kato, S., Y. Yuzawa, N. Tsuboi, S. Maruyama, Y. Morita, T. Matsuguchi, and S. Matsuo. 2004. Endotoxin-induced chemokine expression in murine peritoneal mesothelial cells: the role of toll-like receptor 4. J Am Soc Nephrol 15:1289-1299.

174. Herrick, S. E., and S. E. Mutsaers. 2004. Mesothelial progenitor cells and their potential in tissue engineering. Int J Biochem Cell Biol 36:621-642.

175. Geissmann, F., C. Auffray, R. Palframan, C. Wirrig, A. Ciocca, L. Campisi, E. Narni-Mancinelli, and G. Lauvau. 2008. Blood monocytes: distinct subsets, how they relate to dendritic cells, and their possible roles in the regulation of T-cell responses. Immunol Cell Biol 86:398-408.

176. Marigo, I., L. Dolcetti, P. Serafini, P. Zanovello, and V. Bronte. 2008. Tumorinduced tolerance and immune suppression by myeloid derived suppressor cells. Immunol Rev 222:162-179.

177. Marhaba, R., M. Vitacolonna, D. Hildebrand, M. Baniyash, P. Freyschmidt-Paul, and M. Zoller. 2007. The importance of myeloid-derived suppressor cells in the regulation of autoimmune effector cells by a chronic contact eczema. J Immunol 179:5071-5081.

178. Makarenkova, V. P., V. Bansal, B. M. Matta, L. A. Perez, and J. B. Ochoa. 2006. $\mathrm{CD} 11 \mathrm{~b}+/ \mathrm{Gr}-1+$ myeloid suppressor cells cause $\mathrm{T}$ cell dysfunction after traumatic stress. J Immunol 176:2085-2094.

179. Noseworthy, J. H., C. Lucchinetti, M. Rodriguez, and B. G. Weinshenker. 2000. Multiple sclerosis. N Engl J Med 343:938-952.

180. McQualter, J. L., and C. C. Bernard. 2007. Multiple sclerosis: a battle between destruction and repair. J Neurochem 100:295-306.

181. Weber, M. S., T. Prod'homme, S. Youssef, S. E. Dunn, C. D. Rundle, L. Lee, J. C. Patarroyo, O. Stuve, R. A. Sobel, L. Steinman, and S. S. Zamvil. 2007. Type II monocytes modulate $\mathrm{T}$ cell-mediated central nervous system autoimmune disease. Nat Med 13:935-943.

182. Jung, S., I. Siglienti, O. Grauer, T. Magnus, G. Scarlato, and K. Toyka. 2004. Induction of IL-10 in rat peritoneal macrophages and dendritic cells by glatiramer acetate. J Neuroimmunol 148:63-73.

183. Burger, D., N. Molnarfi, M. S. Weber, K. J. Brandt, M. Benkhoucha, L. Gruaz, M. Chofflon, S. S. Zamvil, and P. H. Lalive. 2009. Glatiramer acetate increases IL-1 receptor antagonist but decreases T cell-induced IL-1beta in human monocytes and multiple sclerosis. Proc Natl Acad Sci U S A 106:4355-4359. 
184. Huang, B., P. Y. Pan, Q. Li, A. I. Sato, D. E. Levy, J. Bromberg, C. M. Divino, and S. H. Chen. 2006. Gr-1+CD115+ immature myeloid suppressor cells mediate the development of tumor-induced $\mathrm{T}$ regulatory cells and T-cell anergy in tumorbearing host. Cancer Res 66:1123-1131.

185. Mannering, S. I., J. S. Morris, K. P. Jensen, A. W. Purcell, M. C. Honeyman, P. M. van Endert, and L. C. Harrison. 2003. A sensitive method for detecting proliferation of rare autoantigen-specific human T cells. J Immunol Methods 283:173-183.

186. Venken, K., M. Thewissen, N. Hellings, V. Somers, K. Hensen, J. L. Rummens, and P. Stinissen. 2007. A CFSE based assay for measuring CD4+CD25+ regulatory $\mathrm{T}$ cell mediated suppression of auto-antigen specific and polyclonal $\mathrm{T}$ cell responses. J Immunol Methods 322:1-11.

187. Talmadge, J. E. 2007. Pathways mediating the expansion and immunosuppressive activity of myeloid-derived suppressor cells and their relevance to cancer therapy. Clin Cancer Res 13:5243-5248.

188. Movahedi, K., M. Guilliams, J. Van den Bossche, R. Van den Bergh, C. Gysemans, A. Beschin, P. De Baetselier, and J. A. Van Ginderachter. 2008. Identification of discrete tumor-induced myeloid-derived suppressor cell subpopulations with distinct T cell-suppressive activity. Blood 111:4233-4244.

189. Phelps, P., and D. J. McCarty, Jr. 1966. Crystal-induced inflammation in canine joints. II. Importance of polymorphonuclear leukocytes. J Exp Med 124:115-126.

190. Issekutz, A. C., and H. Z. Movat. 1980. The in vivo quantitation and kinetics of rabbit neutrophil leukocyte accumulation in the skin in response to chemotactic agents and Escherichia coli. Lab Invest 42:310-317.

191. Numasaki, M., J. Fukushi, M. Ono, S. K. Narula, P. J. Zavodny, T. Kudo, P. D. Robbins, H. Tahara, and M. T. Lotze. 2003. Interleukin-17 promotes angiogenesis and tumor growth. Blood 101:2620-2627.

192. Luo, J. L., S. Maeda, L. C. Hsu, H. Yagita, and M. Karin. 2004. Inhibition of NFkappaB in cancer cells converts inflammation- induced tumor growth mediated by TNFalpha to TRAIL-mediated tumor regression. Cancer Cell 6:297-305.

193. Blanchette, F., and O. Neuhaus. 2008. Glatiramer acetate: evidence for a dual mechanism of action. $J$ Neurol 255 Suppl 1:26-36.

194. Ziemssen, T., and W. Schrempf. 2007. Glatiramer acetate: mechanisms of action in multiple sclerosis. Int Rev Neurobiol 79:537-570.

195. Arreto, C. D., C. Dumarey, M. A. Nahori, and B. B. Vargaftig. 1997. The LPSinduced neutrophil recruitment into rat air pouches is mediated by TNFalpha: likely macrophage origin. Mediators Inflamm 6:335-343.

196. Fujimoto, H., T. Sangai, G. Ishii, A. Ikehara, T. Nagashima, M. Miyazaki, and A. Ochiai. 2009. Stromal MCP-1 in mammary tumors induces tumor-associated macrophage infiltration and contributes to tumor progression. Int J Cancer.

197. Eigenbrod, T., J. H. Park, J. Harder, Y. Iwakura, and G. Nunez. 2008. Cutting edge: critical role for mesothelial cells in necrosis-induced inflammation through the recognition of IL-1 alpha released from dying cells. J Immunol 181:8194-8198.

198. Nakano, K., Y. Okada, K. Saito, R. Tanikawa, N. Sawamukai, Y. Sasaguri, T. Kohro, Y. Wada, T. Kodama, and Y. Tanaka. 2007. Rheumatoid synovial endothelial cells produce macrophage colony-stimulating factor leading to osteoclastogenesis in rheumatoid arthritis. Rheumatology (Oxford) 46:597-603. 
199. Fitzgerald, S. M., D. S. Chi, S. A. Lee, K. Hall, and G. Krishnaswamy. 2004. Inhibition of GM-CSF production in fibroblast-monocyte coculture by prednisone and effects of rhGM-CSF on human lung fibroblasts. Front Biosci 9:342-348.

200. Bischof, R. J., D. Zafiropoulos, J. A. Hamilton, and I. K. Campbell. 2000. Exacerbation of acute inflammatory arthritis by the colony-stimulating factors CSF-1 and granulocyte macrophage (GM)-CSF: evidence of macrophage infiltration and local proliferation. Clin Exp Immunol 119:361-367.

201. Fujita, T., H. Yamabe, M. Shimada, R. Murakami, R. Kumasaka, N. Nakamura, H. Osawa, and K. Okumura. 2008. Thrombin enhances the production of monocyte chemoattractant protein-1 and macrophage inflammatory protein-2 in cultured rat glomerular epithelial cells. Nephrol Dial Transplant 23:3412-3417.

202. Halle, A., V. Hornung, G. C. Petzold, C. R. Stewart, B. G. Monks, T. Reinheckel, K. A. Fitzgerald, E. Latz, K. J. Moore, and D. T. Golenbock. 2008. The NALP3 inflammasome is involved in the innate immune response to amyloid-beta. Nat Immunol 9:857-865.

203. Netea, M. G., C. A. Nold-Petry, M. F. Nold, L. A. Joosten, B. Opitz, J. H. van der Meer, F. L. van de Veerdonk, G. Ferwerda, B. Heinhuis, I. Devesa, C. J. Funk, R. J. Mason, B. J. Kullberg, A. Rubartelli, J. W. van der Meer, and C. A. Dinarello. 2009. Differential requirement for the activation of the inflammasome for processing and release of IL-1beta in monocytes and macrophages. Blood 113:2324-2335.

204. Hansen, K., and B. T. Mossman. 1987. Generation of superoxide (O2-.) from alveolar macrophages exposed to asbestiform and nonfibrous particles. Cancer Res 47:1681-1686. 


\section{Appendix}

\section{Appendix 1: $\mathrm{GA}^{+}$cells in the blood show monocyte cell surface phenotype}
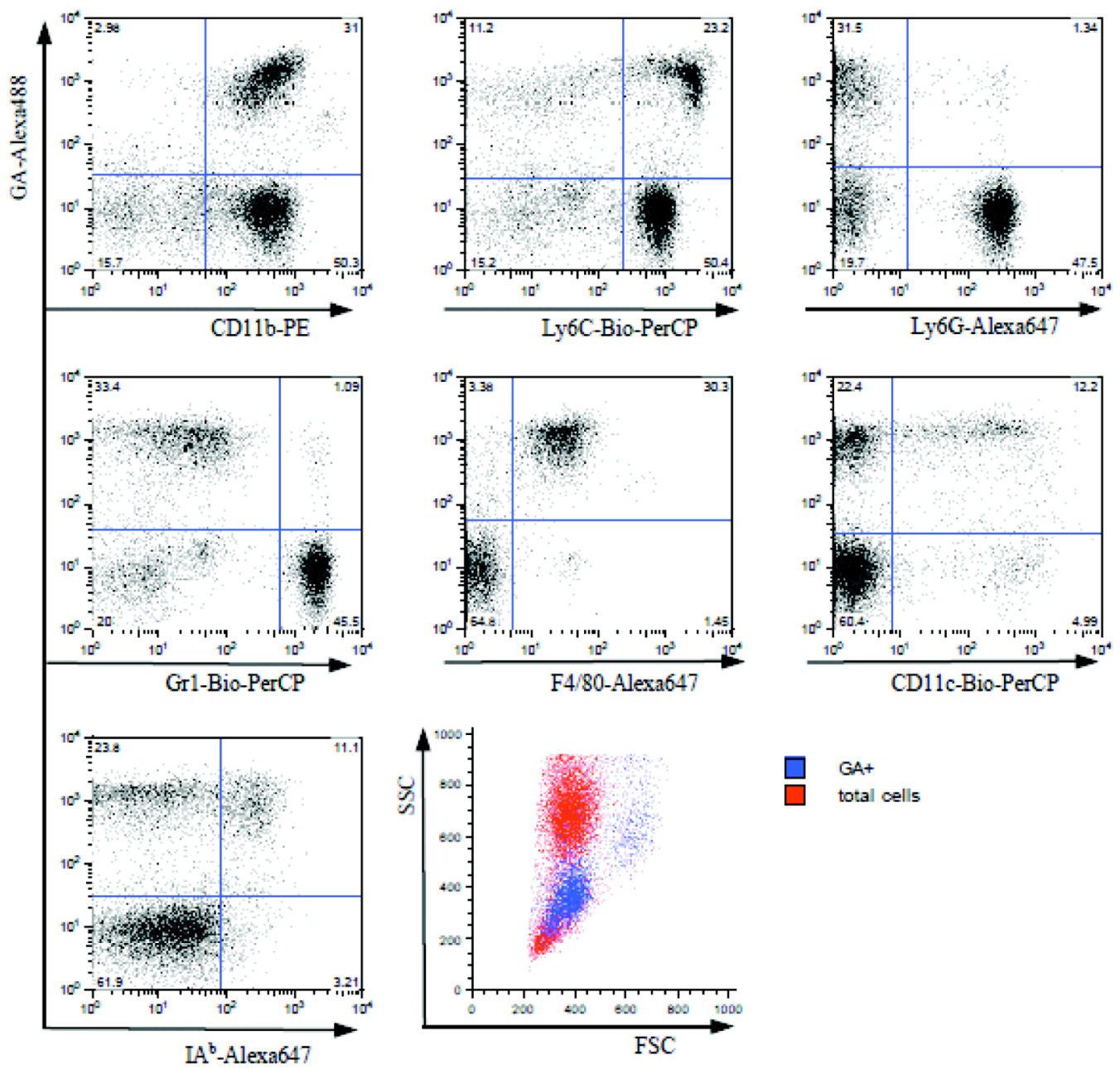

$\square$ GA+

$\square$ total cells

GA-A488 was injected intravenously to $\mathrm{C} 57 \mathrm{Bl} / 6 \mathrm{~J}$ mice at a dose of $50 \mu \mathrm{g} / \mathrm{mouse}$. CD11b $\mathrm{b}^{\text {hi }}$ cells were enriched from pooled blood of five mice by magnetic sorting one hour after the injections. The cells were then stained for cell surface markers and analysed by flow cytometry. Bottom right panel row shows the FSC/SSC profile of $\mathrm{GA}^{+}$cells in comparison to total blood cells. Data represents one of two experiments. Appendix 1 was provided by Aras Toker. 
Appendix 2: Circulating monocytes display enhanced suppressor abilities of $\mathbf{T}$ cell expansion in vitro.

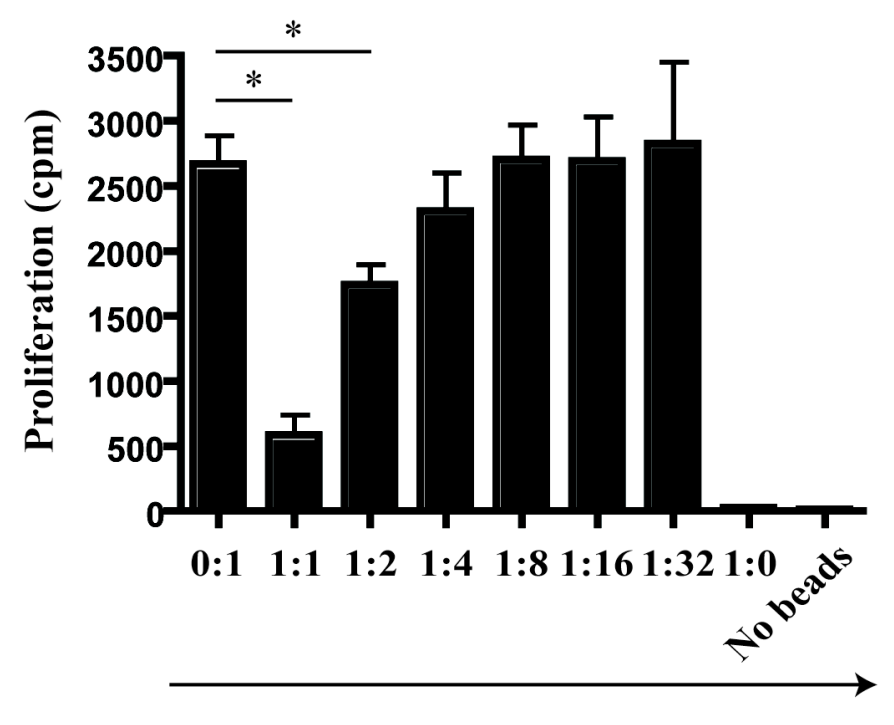

CD11b:Splenocyte ratio

Peripheral blood mononuclear cells were recovered from naïve C57B1/6J mice and the CD11 b ${ }^{\text {hi }}$ monocytes enriched by magnetic sorting $\left(>80 \% \mathrm{CD} 11 \mathrm{~b}^{\mathrm{hi}}\right)$. The monocytes were co-cultured with $5 \times 10^{4} \mathrm{C} 57 \mathrm{Bl} / 6 \mathrm{~J}$ splenocytes at the indicated ratios in the presence of DynaBead ${ }^{\circledR} \mathrm{CD} 3 / \mathrm{CD} 28 \mathrm{~T}$ cell expander beads in 96 well round-bottom plates. The cells were incubated fro 20 hours, and an additional eight hours in the presence of $\left[{ }^{3} \mathrm{H}\right]$-thymidine. T cell expansion was measured by $\left[{ }^{3} \mathrm{H}\right]$-thymidine incorporation. Measurements performed in triplicate. Values represent mean S.E.M. ${ }^{*} P<0.01$ as determined by two-tailed unpaired Student test. Results are representative of two independent experiments with five mice per group. Appendix 2 was provided by Aras Toker. 